This item was submitted to Loughborough's Research Repository by the author.

Items in Figshare are protected by copyright, with all rights reserved, unless otherwise indicated.

\title{
Selective Laser Melting (SLM) of gold (Au)
}

PLEASE CITE THE PUBLISHED VERSION

PUBLISHER

(c) Mushtaq Khan

LICENCE

CC BY-NC-ND 4.0

REPOSITORY RECORD

Khan, Mushtaq. 2019. "Selective Laser Melting (SLM) of Gold (au)". figshare. https://hdl.handle.net/2134/6163. 
This item was submitted to Loughborough's Institutional Repository (https://dspace.lboro.ac.uk/) by the author and is made available under the following Creative Commons Licence conditions.

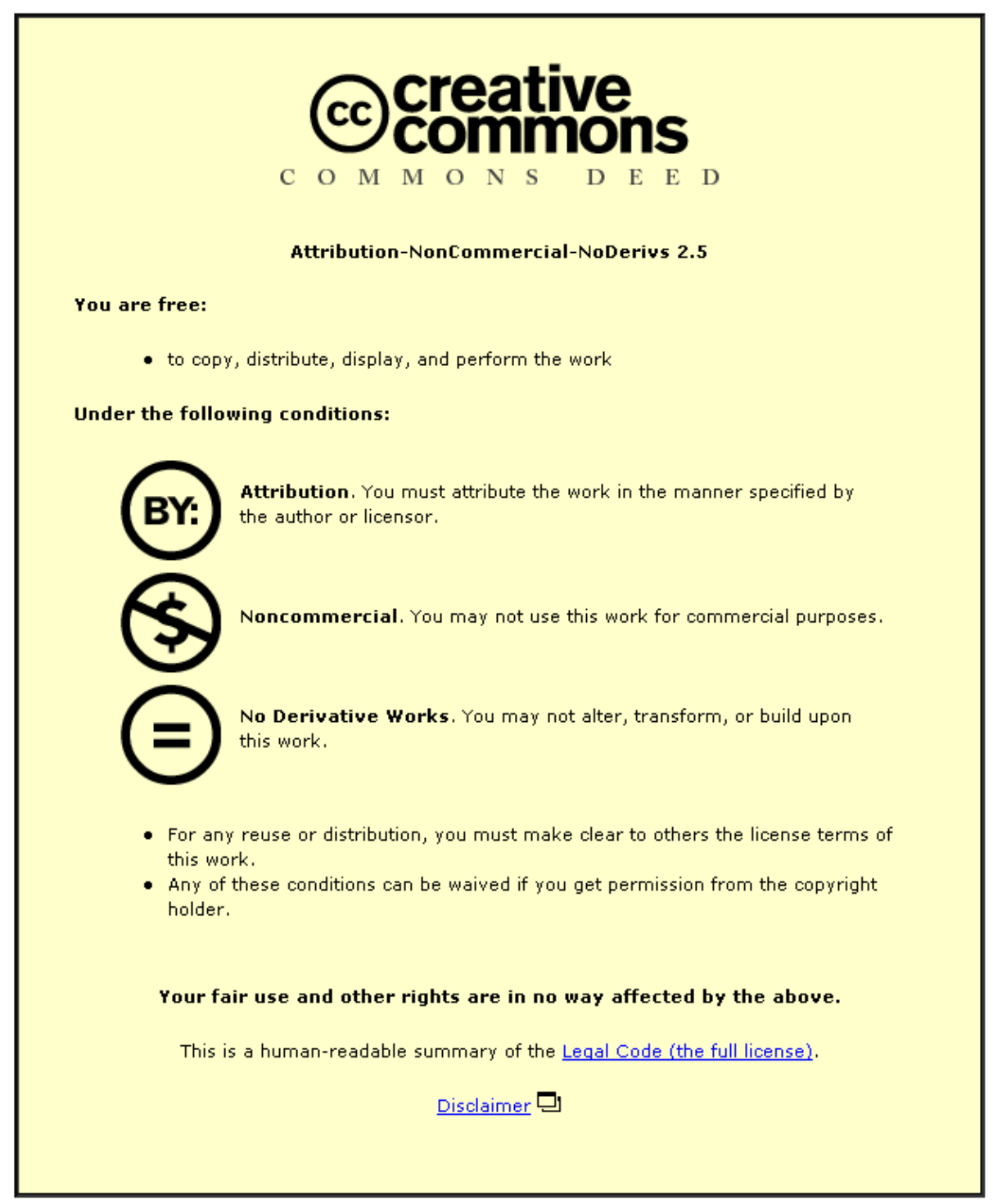

For the full text of this licence, please go to: http://creativecommons.org/licenses/by-nc-nd/2.5/ 


\section{Selective Laser Melting (SLM) of Gold (Au)}

By

\section{Mushtaq Khan}

BSc MSc (Mechanical Engineering)

Doctoral thesis submitted in partial fulfilment of the requirements of the award of Doctor of Philosophy of Loughborough University

January 2010

(C) by Mushtaq Khan (2010) 


\section{Abstract}

Selective Laser Melting (SLM) is a laser based Solid Freeform Fabrication (SFF) process which uses laser energy to melt a thin layer of metal powder. This process is repeated to produce a 3-dimensional metallic part. SLM is capable of producing intricate parts which are otherwise difficult to produce with conventional manufacturing techniques. As compared to traditional manufacturing processes, SLM can also produce parts with higher density. Before a material is processed using SLM, suitable processing parameters are first identified. Over the years, different materials have been processed using the SLM process. However, very little work has been done on SLM of bio-compatible precious metals such as gold and its alloys. Gold and its alloys have been used for manufacturing of dental crowns for centuries. The SLM process could be used to produce intricate metallic substructures for porcelain fused to metal dental restorations.

This research work was focused on understanding the processing parameters for SLM of 24 carat gold powder. The gold powder was analyzed for Particle Size Distribution (PSD), apparent density and tap density before identifying suitable processing parameters for SLM. The gold powder particles were found to be spherical in nature but smaller particles stuck to each other and formed larger powder agglomerates. From the apparent density experiments, the gold powder was found to be cohesive and non-flowing in nature which hindered powder flowability during the powder deposition process with the existing system. This issue was resolved by designing a new powder deposition system which could allow the gold powder to flow evenly over the substrate. The tap density of the gold powder was found by Constant Weight Tap Density (CWTD) and Constant Volume Tap Density (CVTD) techniques. The difference in results from these two techniques was negligible. Diffuse Reflectance Infrared Fourier Transform Spectroscopy (DRIFTS) of gold powder showed it to be more than twice as reflective as other commonly processed metal powders such as stainless steel and H13 tool steel. This analysis proved useful in understanding the laser processing of gold powder. Due to the high cost and small quantity of material available for this work, a very small build platform was designed to optimize material utilization and reduce wastage. Single scans were performed on a single layer of gold powder to identify the good melting region. Five different regions i.e. balling, good melting, unstable melt, weak sintering and very little sintering were observed in the processing window. The balling phenomenon was observed at low and high scan speeds, which was due to the melt pool instability at these parameter settings. The size of droplets (balling) also increased with decreasing scan speed and increasing laser power which was 
due to an increase in the break up time of the molten metal. In the good melting region, the gold powder was found to be completely melted and continuous beads were successfully produced. The unstable melt region showed the melt pool spreading unevenly in different directions whereas in the weak sintering and very little sintering regions the gold powder did not melt completely. Single layers were produced on a layer of gold powder, which showed the parameters in the good melting regions to be suitable for multiple layer parts manufacturing.

Gold cubes were produced using the suitable processing parameters identified from single scan and single layer experiments and then analyzed for their internal porosity. The porosity in the gold cubes was found to be at a minimum for parameters obtained in the good melting region. The internal porosity was found to be mostly inter-layer porosity; this indicated less heat transferred to the region between the two layers which could be associated with the high reflectivity of gold. The inter-layer porosity in gold cubes was further reduced by reducing the layer thickness. This could be due to the thinner layers requiring less energy to melt and be fused to the previous layers. The hatch distance had a negligible effect on the inter-layer porosity of gold cubes. The reduction in hatch distance increased the energy delivered but it was still not enough to completely melt the gold powder and fuse it to the previous layer. A pre-scan technique was also tested to be used for pre-heating the powder bed. However, due to the rapid drop in temperature, this technique was not found suitable to be used as a powder bed pre-heating technique. The gold cubes were checked for their mechanical properties i.e. hardness and modulus. The hardness of gold cubes was found to be higher than expected for 24 carat gold. The modulus was found to be less than 24 carat gold. This variation in the mechanical properties of gold cubes could be due to the rapid heating and cooling of material during the laser processing or presence of internal porosity in these gold cubes.

After single scans and single layers manufacturing, gold dental parts (premolar and molar) were also manufactured using the optimum processing parameters. These gold dental parts were also analyzed for their internal porosity, which was found to be less than that observed in gold cubes. This difference in porosity could be due to the difference in structure of gold cubes and premolar part, where the latter was a thin wall structure. 


\section{Acknowledgments}

I would like to extend my gratitude to my supervisor Professor Phill Dickens for his guidance and encouragement throughout my research. I would also like to thank him for his efforts in arranging funding for my stay in Loughborough and to complete my $\mathrm{PhD}$ in time.

Thanks to technical staff Mark East, Phil Brindley and Mark Hardy for their efforts. Mr Peter Wileman for his advice on laser issues. Mr Andy Sandaver and Jagpal Singh for their help in specimen preparation and testing. Thanks to all members of the Additive Manufacturing Research Group (AMRG) at Loughborough especially Kamran Mumtaz and Helen Thomas for his valuable input and assistance.

Thanks go to my family, especially my father Prof Dr. Nawar Khan (Abboo), my mother (Ammi), Nayab Khan (Wife) and Ashfaq Khan (Brother) who supported me and encouraged me through difficult times and also my other family members: Azra, Aftab, Samad and Sagheer. I am also thankful to my little son Bilal Khan for not disturbing me in the final stage of my $\mathrm{PhD}$.

My final thanks go to my friends especially Aamer Gohar Salarzai (Lala), Khurshid Alam and Zahid Rizwan Khokhar who helped me in difficult times and facilitated my stay in UK.

Mushtaq Khan

January 2010 


\section{Table of Contents}

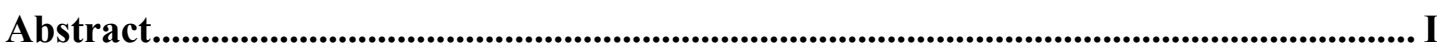

Acknowledgments ….............................................................................................................................. III

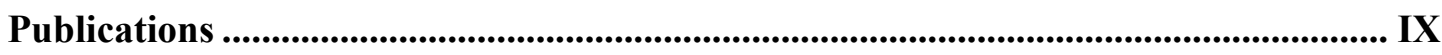

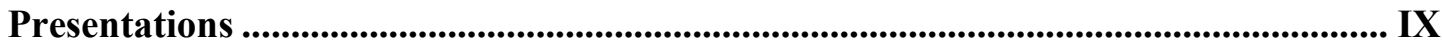

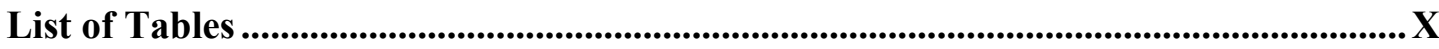

List of Figures................................................................................................................................... XI

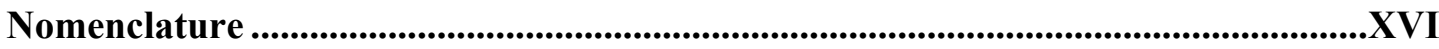

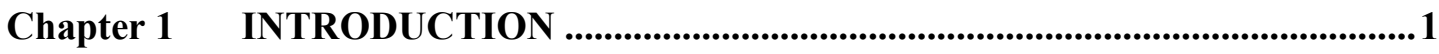

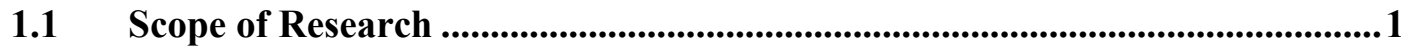

1.2 Thesis Outline ..................................................................................................................... 3

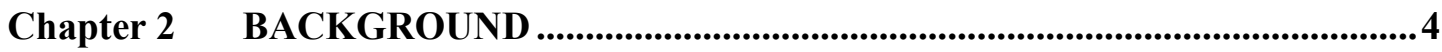

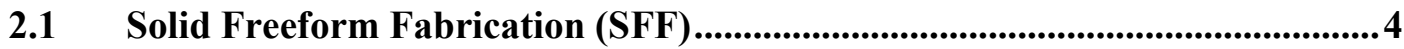

2.2 Solid Freeform Fabrication (SFF) of Metals ...............................................6

2.2.1 Key Non-Laser Metal Processing Technologies ............................................. 6

2.2.1.1. Electron Beam Melting (EBM) ............................................................. 7

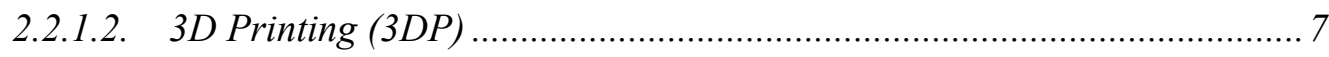

2.2.1.3. Ultrasonic Consolidation (UC) …...................................................... 7

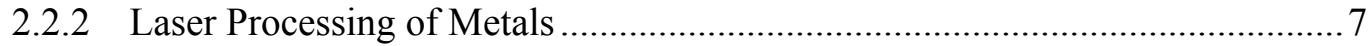

2.2.2.1. Sintering mechanisms …................................................................ 9

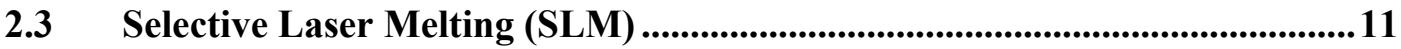

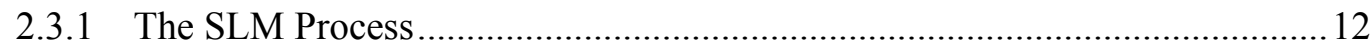

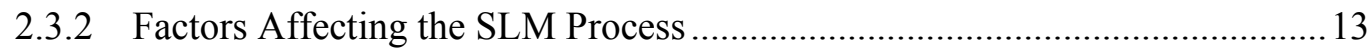

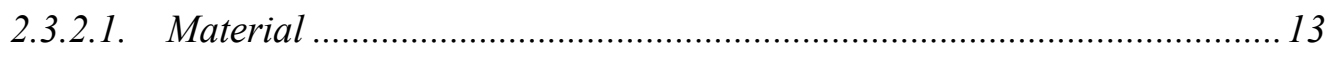

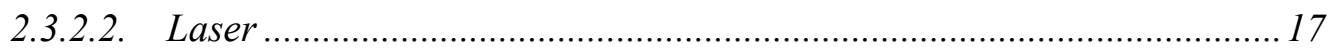

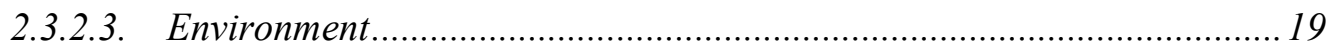

2.3.3 Thermodynamics of the SLM Process ............................................................ 19

2.3.3.1. Laser Material Interaction................................................................. 19

2.3.3.2. Heat Transfer in the SLM Process Powder Bed ....................................23

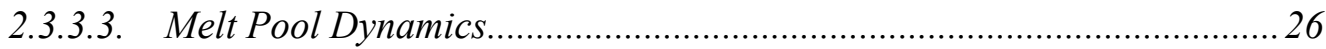

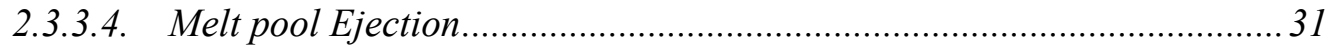

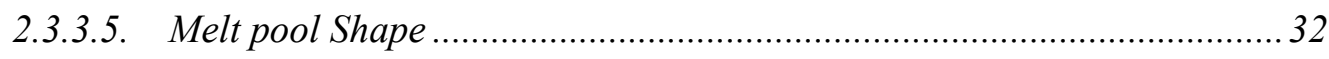




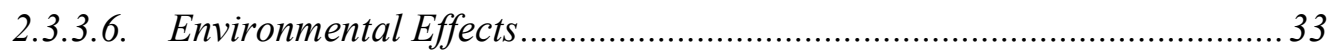

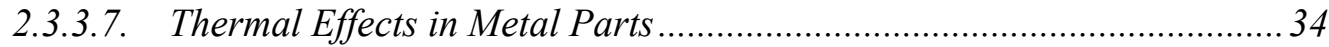

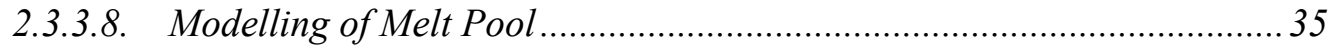

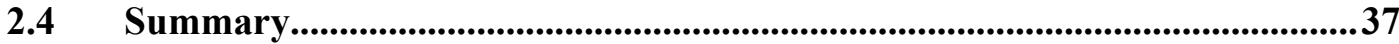

Chapter 3 DIGITAL MANUFACTURING IN MEDICINE AND DENTISTRY .38

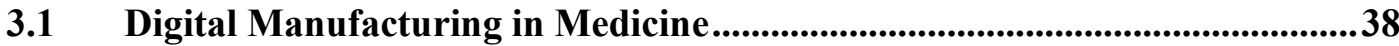

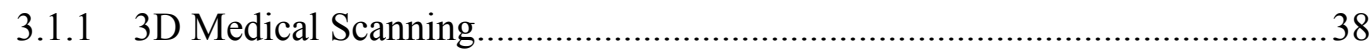

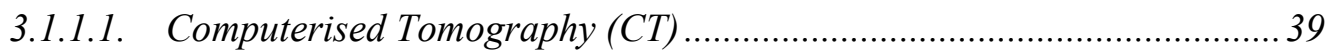

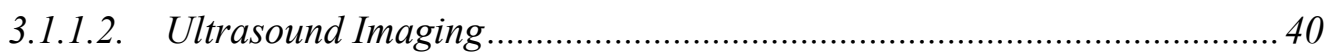

3.1.1.3. Magnetic Resonance Imaging (MRI) ................................................. 40

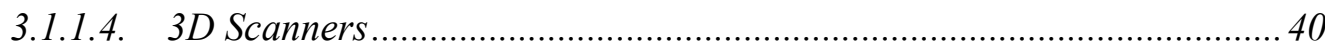

3.2 Rapid Prototyping (RP) and Rapid Manufacturing (RM) in Medicine .......40

3.3 Digital Manufacturing in Dentistry .................................................................44

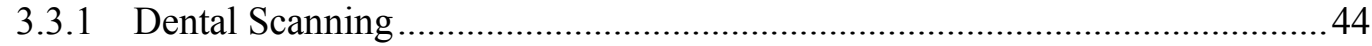

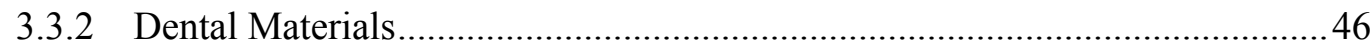

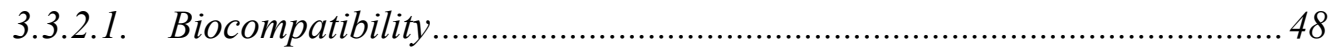

3.3.2.2. Tarnish and Corrosion Resistance .......................................................... 48

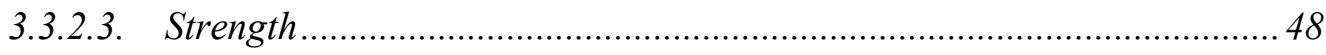

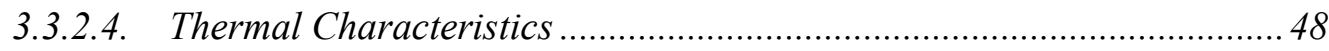

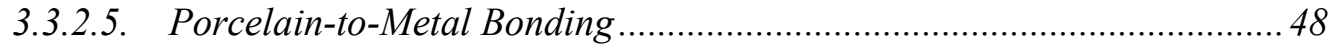

3.3.3 Existing Manufacturing Systems in Dentistry .............................................49

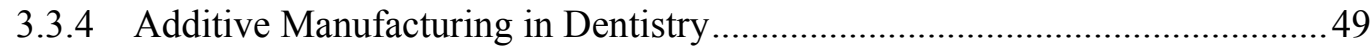

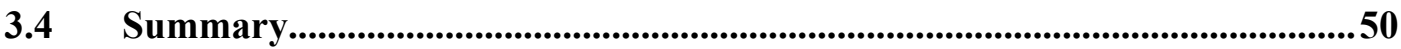

Chapter 4 HYPOTHESIS AND RESEARCH OBJECTIVES .............................51

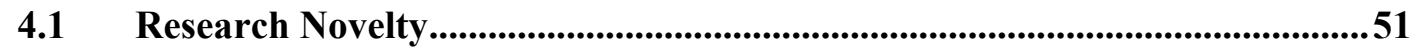

$4.2 \quad$ Research Aim and Objectives ....................................................................................52

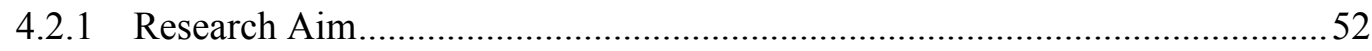

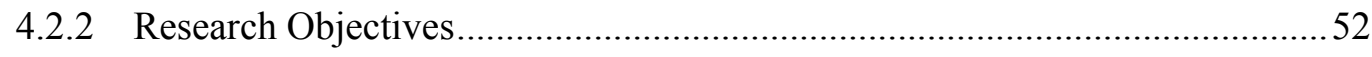

4.3 Research Methodology ........................................................................................5

4.3.1 Material Selection and Characterisation ......................................................53

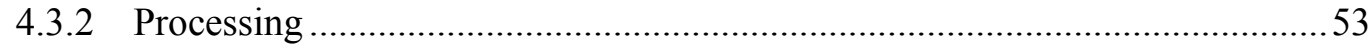

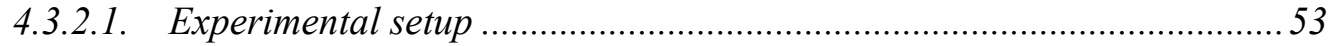

4.3.2.2. Identifying suitable processing parameters .........................................53

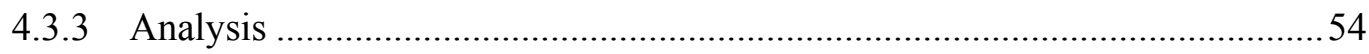


Chapter 5 LASER MATERIAL INTERACTION ..................................................55

5.1 Radiation Absorption of Metal Powders ...........................................................55

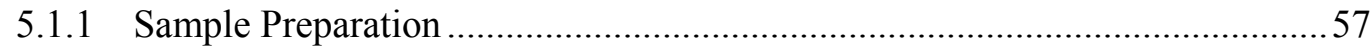

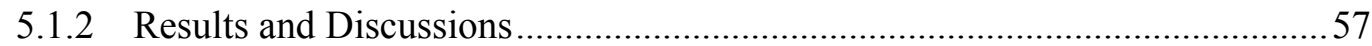

5.1.2.1. Effect of Particle Size on Radiation Absorption ...................................58

5.1.2.2. Effect of Particles Packing on Radiation Absorption .............................. 59

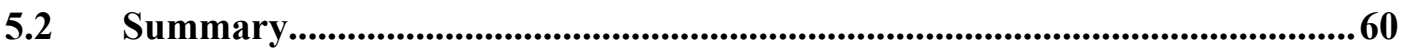

Chapter 6 CHARACTERISTICS OF THE POWDER........................................62

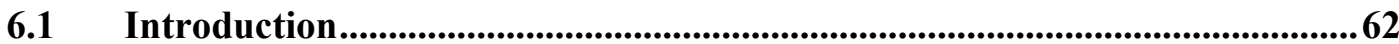

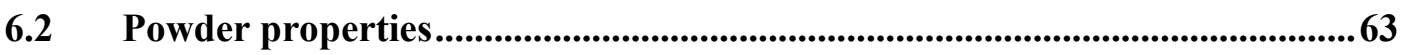

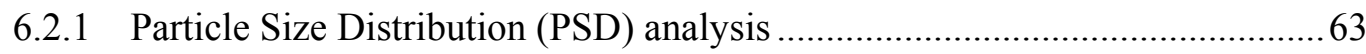

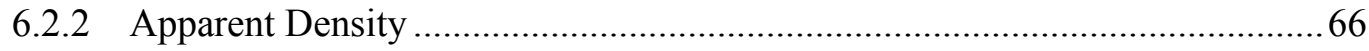

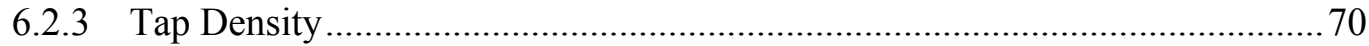

6.2.3.1. Constant Weight Tap Density (CWTD) Method .................................... 71

6.2.3.2. Constant Volume Tap Density (CVTD) Method .................................... 72

6.2.4 Hausner Ratio (HR) and Compressibility of Gold (Au) Powder......................75

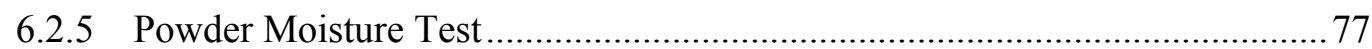

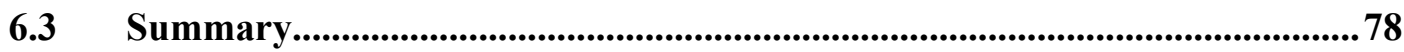

Chapter 7 CHARACTERISTICS OF PROCESSING SYSTEM............................80

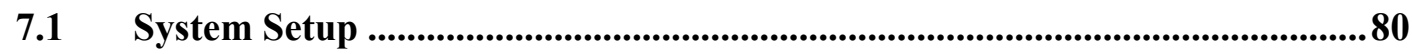

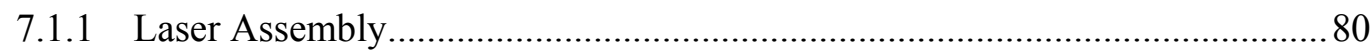

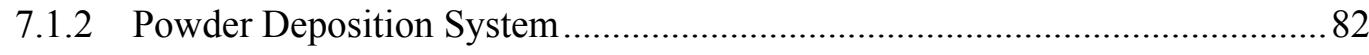

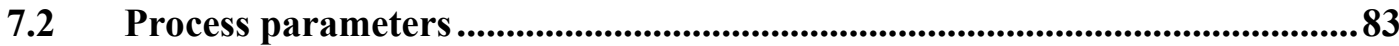

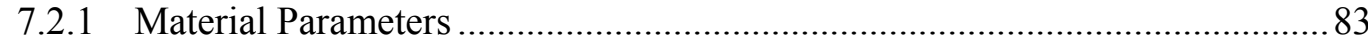

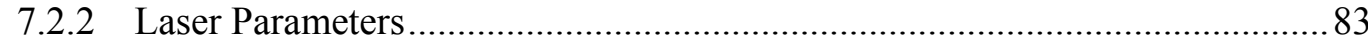

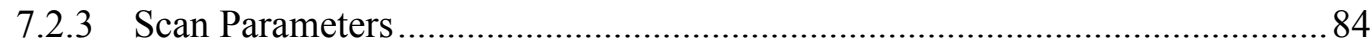

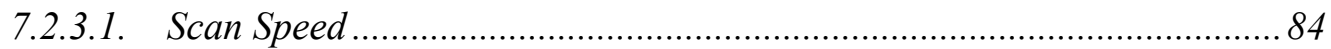

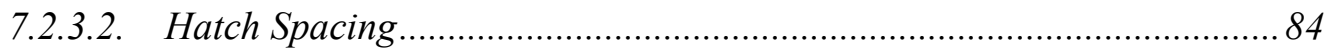

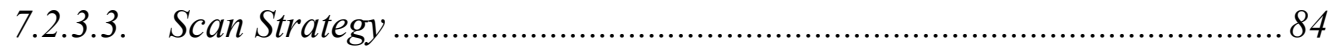

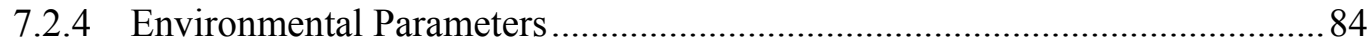

7.3 Laser Spot Size Measurements .................................................................................85

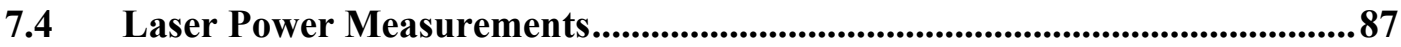

7.5 Modifications to the SLM 100 System ...................................................................89

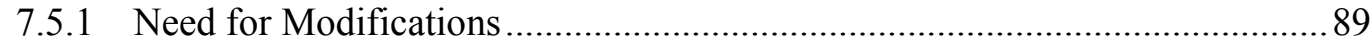




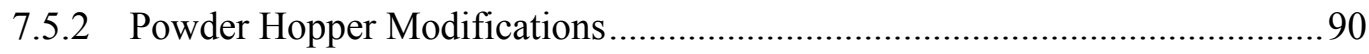

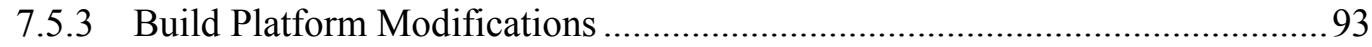

7.5.4 Prevention of Contamination of Gold Powder ................................................99

7.5.5 Layer Thickness Control for the New Setup ….............................................95

7.5.6 Wiper Rotation Control for the New Setup .....................................................95

7.5.7 Bed Temperature of the New Build Platform................................................95

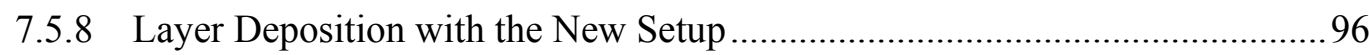

7.6 Summary...................................................................................................................96

Chapter 8 SELECTIVE LASER MELTING (SLM) OF GOLD POWDER .........97

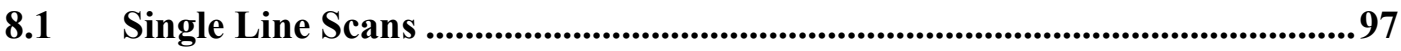

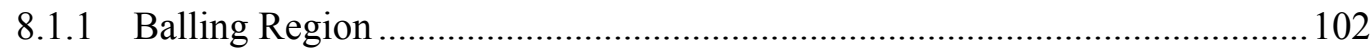

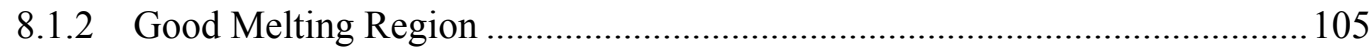

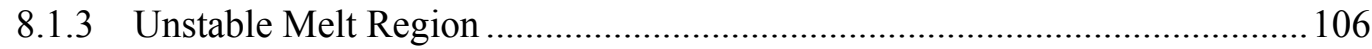

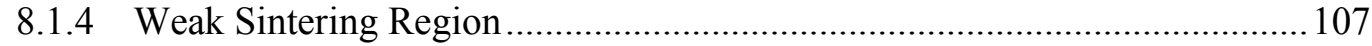

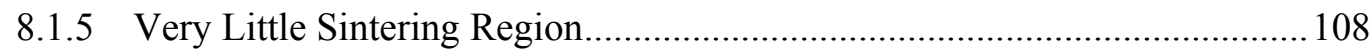

8.2 Splitting of the Melt Pool..............................................................................109

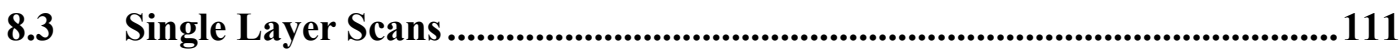

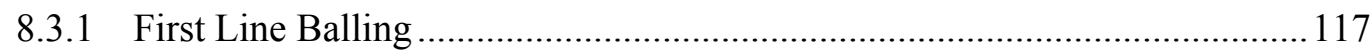

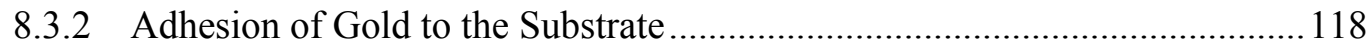

8.4 Manufacturing of Gold Cubes .................................................................................. 119

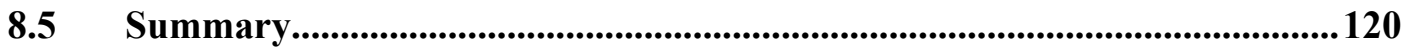

Chapter 9 ANALYSIS OF GOLD CUBES.................................................................. 122

9.1 Porosity Measurement of Gold Cubes .............................................................122

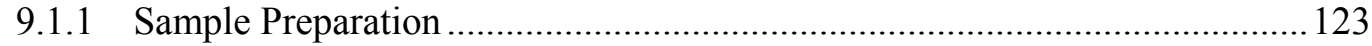

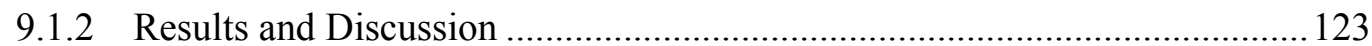

9.2 Improvement in the Density of Gold Cubes ..................................................... 127

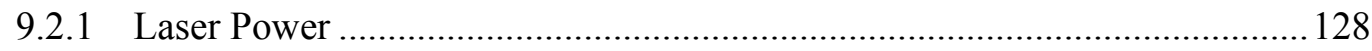

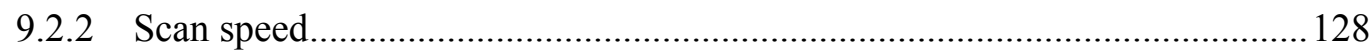

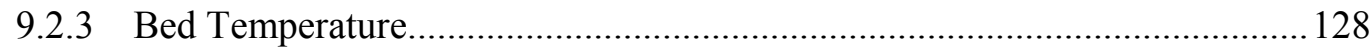

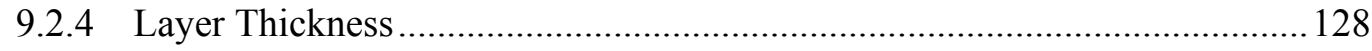

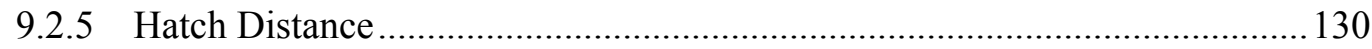

$9.3 \quad$ Intra Layer Porosity .................................................................................................. 130

9.4 Optimum Processing Parameters.................................................................. 131

9.5 Temperature Measurements in Gold Powder Bed ....................................... 132 


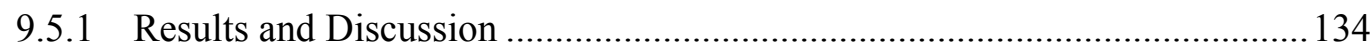

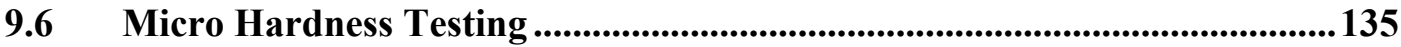

9.7 Nanoindentation of Gold Cubes .......................................................................... 136

9.7.1 Selection of Maximum Load ....................................................................... 136

9.7.2 Selection of Loading Rate and Holding Time ........................................... 137

9.7.3 Elastic Modulus of Gold Cubes ..................................................................... 138

9.8 Manufacturing of Gold Dental Parts ..........................................................138

9.9 Computerised Tomography (CT) Scan of the Dental Parts .......................... 140

9.10 Porosity Measurement of Gold Dental Part ................................................ 142

9.11 Summary ........................................................................................................................ 144

\section{Chapter 10 CONCLUSIONS AND RECOMMENDATIONS FOR FUTURE} WORK 145

10.1 Material Characterisation............................................................................... 145

10.1.1 Reflectivity of Gold Powder ................................................................. 145

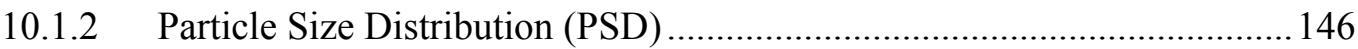

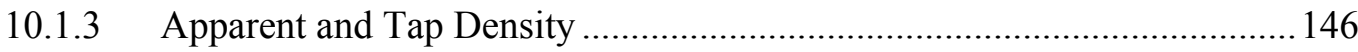

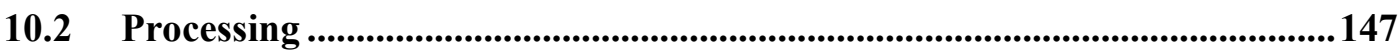

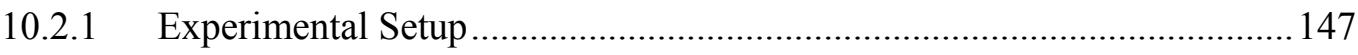

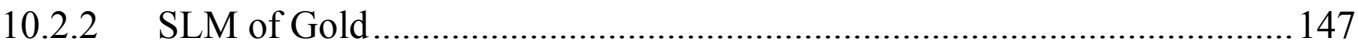

10.2.3 Manufacturing of Gold Dental Parts....................................................... 149

10.3 Analysis ................................................................................................................ 149

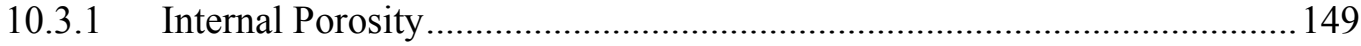

10.3.2 Mechanical Properties.............................................................................. 149

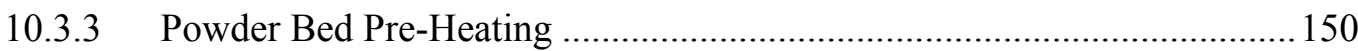

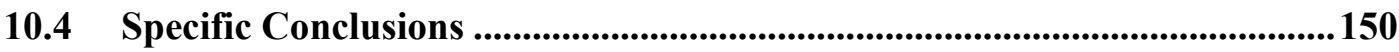

10.5 Recommendations for Future Work ............................................................152

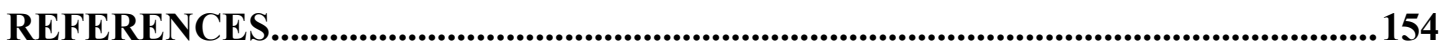

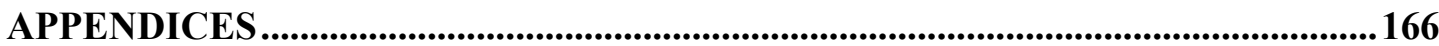

Appendix-A............................................................................................................................. 166

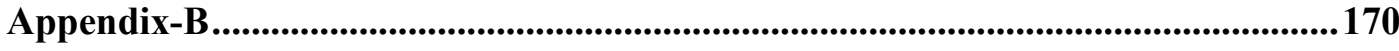




\section{Publications}

- M. Khan and P. Dickens (2008), Processing Parameters for Selective laser Melting (SLM) of Gold, The Proceedings of $19^{\text {th }}$ International Solid Freeform Fabrication Symposium, pp. 278-289 , 4-6 August, Austin, Texas, USA

- M. Khan and P. Dickens (2009), Selective Laser Melting (SLM) of 24 Carat Gold $(A u)$, The Proceeding of $5^{\text {th }}$ international conference on Gold Science, Technology and its Applications, 26 - 29 July, Heidelberg, Germany

- Khan, M. and Dickens, P. (2009), Selective Laser Melting (SLM) of Gold (Au),Rapid Prototyping Journal [Submitted]

- Khan, M. and Dickens, P. (2010), Selective Laser Melting (SLM) of 24 Carat Gold, Journal Gold Bulletin [Accepted to be published in June 2010]

\section{Presentations}

- Wolfson School Research Student Conference, February 2008, Loughborough University, Loughborough, UK.

- 19th International Solid Freeform Fabrication (SFF) Symposium, August 2008, Austin, Texas, USA 


\section{List of Tables}

Table 2-1: Absorption coefficients (Kabs) of different materials in powder and bulk

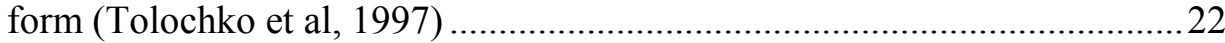

Table 3-1: Some of the commercially available CAD/ CAM systems in dentistry .........49

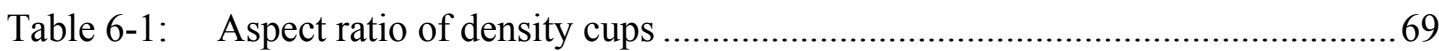

Table 6-2: Apparent density, tap density and compressibility for different size density

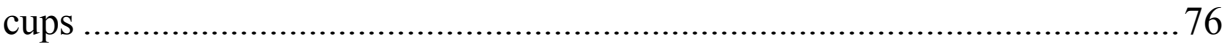

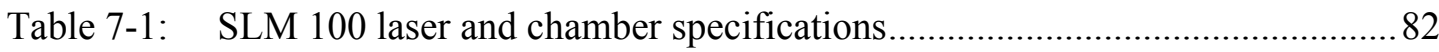

Table 7-2: Input Power, the resulting input current and the optical power output for the

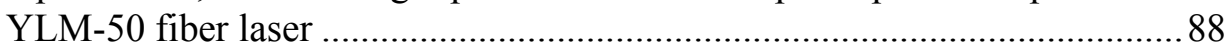

Table 8-1: Laser parameters and their range for single scan experiments.......................97

Table 8-2: Experimental parameters for SLM of single layers of gold ......................... 111

Table 9-1: Parameters and their values for gold cubes manufacturing........................... 124

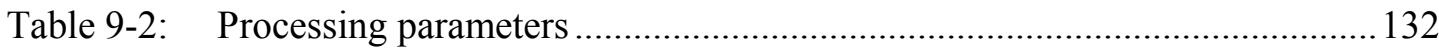

Table 9-3: Processing parameters for temperature measurements ............................... 133 


\section{List of Figures}

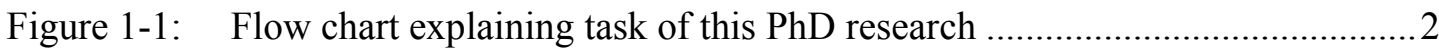

Figure 2-1: Different sectors embracing SFF technologies (Wohlers, 2008) ................... 4

Figure 2-2: Classification of SFF technologies ..........................................................

Figure 2-3: Lattice spatial structure fabricated from stainless steel grade $316 \mathrm{~L}$

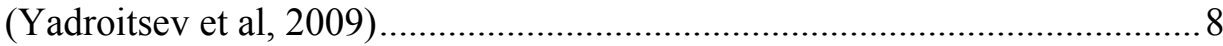

Figure 2-4: SLM fabricated heat sinks, base dimensions $50 \mathrm{~mm} \times 100 \mathrm{~mm}$, flow in the $\mathrm{z}$ direction. (a) Pin fin 6061, (b) Rectangle, (c) Rect RND, (d) Ellipse, (e)

Lattice and (f) close up of Lattice. .......................................................... 8

Figure 2-5: (Left) Neck formation in SSS, (Right) Neck formation between two stainless steel particles (Van Der Schueren, 1996)......................................................

Figure 2-6: Liquid Phase Sintering (LPS) of WC-Co powder mixture, left: before infiltration (a: non-molten WC particle, b: molten Co, c: porosity), right: after infiltration with copper (Kruth et al, 2005)

Figure 2-7: Cross section of a Ti part produced by Selective Laser Melting (Kruth et al, 2005) 11

Figure 2-8: Schematics of the SLM process ............................................................... 13

Figure 2-9: (a-c) Change in absorption coefficient of Nickel alloy (PG12N02) during laser treatment with a radiation power density of 100 (a and c) and $250 \mathrm{~W} / \mathrm{cm} 2$ (b) (Tolochko et al, 1997)

Figure 2-10: (a) Transfer of heat between powder particles via conduction \& (b) heat distribution within powder bed (Mumtaz, 2008) .......................................24

Figure 2-11: Energy loss during laser material processing in powder .............................25

Figure 2-12: Three phase equilibrium of wetting liquid on a solid (adapted from Eustathopoulos et al, 1999).

Figure 2-13: (a) Small spheres attached to the side of melt pool (b) Melt pool splitting into larger droplets

Figure 2-14: Transition form half cylinder to sphere (adapted from (Kruth et al, 2004)). 28

Figure 2-15: Schematics presentation of Marangoni convection in a melt due to the presence of a surface tension gradient (d $\gamma \mathrm{LV} / \mathrm{dT})$ (Mills et al, 1998)

Figure 2-16: Marangoni convection within the cross-section of the melt pool (Mumtaz, 2008)

Figure 2-17: Melt pool ejection as a result of high recoil pressure on melt pool surface (Mumtaz, 2008). 32

Figure 2-18: Melt pool shape modification due to recoil pressure ....................................33

Figure 3-1: 3D reconstruction and implant design in MIMICS (Gibson, 2005) ..............39

Figure 3-2: (a) Osteotomy (bone cutting) planning and (b) mandible reconstruction on a RP model (Gibson, 2005).

Figure 3-3: Model of a patient's skull made by two-colour stereolithography indicating the shape and position of a tumour (Vandenbroucke, 2008)

Figure 3-4: Hearing aids by Materialise (a) hearing aid design (b) RP model (Materialise, 2009)

Figure 3-5: (a) Virtual planning of a complex spinal surgery using a personalized drill guide for spinal screw insertion (b) SLA made drill guide being used during spinal screw placement in a lumbar vertebra (Brussel, 2002) 
Figure 3-6: (a) Cranioplasty plate for skull reconstruction made by SLM of titanium (Source: (MCP HEK, 2009)); (b)Tri-flange implant for hip replacement surgery made by EBM of titanium (Source: (Arcam, 2009))

Figure 3-7: Digital digitizers/ scanners (a) InciseTM Scanner (Renishaw, 2009), (b) Laserdenta scanner (Laserdenta, 2009) and (c) CEREC $®$ acquisition centre (CEREC, 2009)

Figure 3-8: Process flow of digital dental restoration process........................................46

Figure 3-9: Cross section of a porcelain fused to gold crown (Cascone, 2003) ..............47

Figure 3-10: (a) SLM made cobalt-chromium bridges by the Medifacturing system from BEGO (BEGO, 2009) and (b) by infiDent system from Sirona (Sirona, 2009)

Figure 5-1: Radiation absorption and reflection from a powder bed...............................55

Figure 5-2: Different components of reflected radiations from the metal powders ........56

Figure 5-3: (a) DRIFTS equipment with (b) Selector diffuse reflectance accessory .......56

Figure 5-4: Percentage reflectance of different metal powders ......................................58

Figure 5-5: Reflectance of copper powder with different particle sizes..........................59

Figure 5-6: Reflectance of gold powder packed with different densities .........................60

Figure 6-1: $\quad$ PSD of gold $(\mathrm{Au})$ powder in water: $\mathrm{d}(0.1)=23.163, \mathrm{~d}(0.5)=51.296$ and $\mathrm{d}(0.9)=88.184$

Figure 6-2: $\quad$ PSD of gold $(\mathrm{Au})$ powder in isopropanol: $\mathrm{d}(0.1)=10.43, \mathrm{~d}(0.5)=24.55$ and $\mathrm{d}(0.9)=46.73$. .64

Figure 6-3: (a-d) SEM Images of ARGENC gold (Au) powder .....................................66

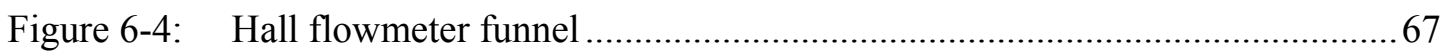

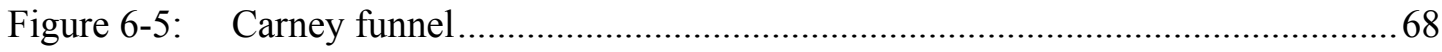

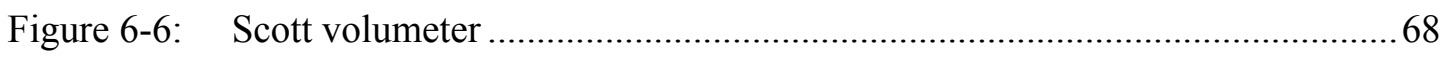

Figure 6-7: Apparent density cups with different aspect ratios ..................................... 70

Figure 6-8: Apparent density with different aspect ratio density cups ........................... 70

Figure 6-9: Standard $25 \mathrm{~cm} 3$ graduated glass cylinder............................................... 71

Figure 6-10: Tap density of gold powder with CWTD method ........................................ 72

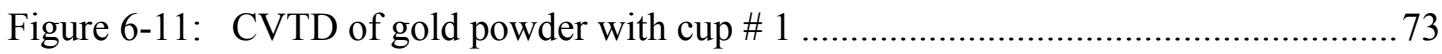

Figure 6-12: CVTD of gold powder with cup \# 2 ........................................................... 74

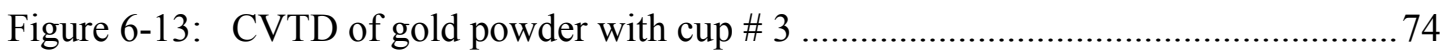

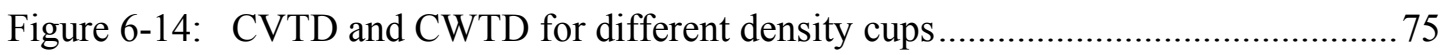

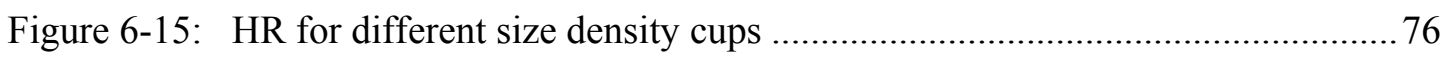

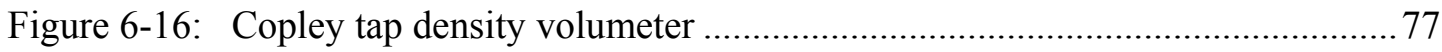

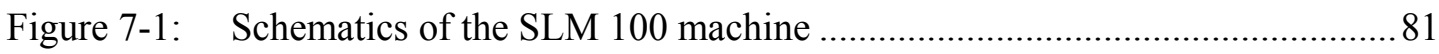

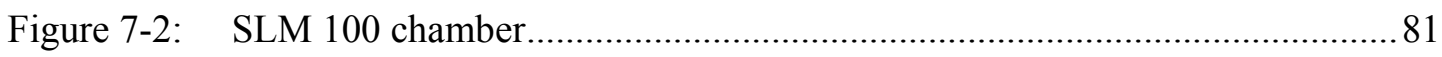

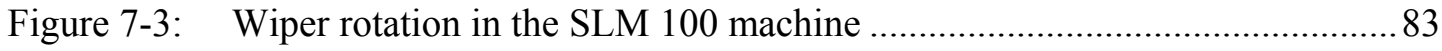

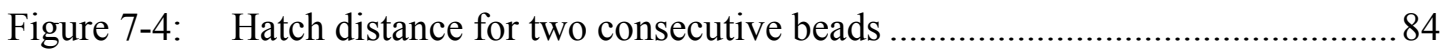

Figure 7-5: (a) Template and (b) Black burn paper taped to the template........................85

Figure 7-6: Spot size measurement $(35 \mu \mathrm{m})$ for YLM-50 laser on black burn paper ...... 85

Figure 7-7: Laser spot size vs position of the piston ................................................ 87 
Figure 7-8: Laser spot size variation with laser power for different piston levels from -

$5 \mathrm{~mm}$ to $+5 \mathrm{~mm}$.

Figure 7-9: Input current and laser output power

Figure 7-10: Rectangular hopper (a) CAD model with complete installation assembly (b) FDM model 90

Figure 7-11: Circular hopper and the installation assembly 91

Figure 7-12: Selected smaller hopper (a) disassembled (b) assembled (c) hopper assembled and installed inside the SLM 100 chamber 92

Figure 7-13: Original SLM 100 hopper. .92

Figure 7-14: New platform (a) smaller piston and (b) build platform with bore. .93

Figure 7-15: Arrangement of the new piston, base and hopper for the reduced build platform 94

Figure 7-16: Plastic floor in the chamber with new deposition system and smaller build platform. .95

Figure 7-17: Layer deposition with the new deposition system and build platform .........96

Figure 8-1: Different processing regions for SLM of gold powder for first set of experiments

Figure 8-2: Complete processing window for SLM of gold powder 101

Figure 8-3: Balling at $50 \mathrm{~W}$ laser power and $25 \mathrm{~mm} / \mathrm{s}$ scan speed. 102

Figure 8-4: (a-x) Balling, unstable melt and weak sintering at different laser power and scan speeds (all images were taken at same magnification). 104

Figure 8-5: Good melting at 50W laser power and $65 \mathrm{~mm} / \mathrm{s}$ scan speed ....................... 106

Figure 8-6: Unstable melt at 50W laser power and $45 \mathrm{~mm} / \mathrm{s}$ scan speed ...................... 107

Figure 8-7: Weak sintering at $15 \mathrm{~W}$ laser power and $25 \mathrm{~mm} / \mathrm{s}$ scan speed ..................... 108

Figure 8-8: Very little sintering at $10 \mathrm{~W}$ laser power and $10 \mathrm{~mm} / \mathrm{s}$ scan speed ............. 109

Figure 8-9: Microscope image of single line scan of gold: melt pool splitting at $25 \mathrm{~W}$ laser power and $25 \mathrm{~mm} / \mathrm{s}$ scan speed 110

Figure 8-10: Heat transfer and temperatures during the laser melting process ..... 110

Figure 8-11: Single layers scanned with (a) $80 \mu \mathrm{m}$ hatch distance (b) $100 \mu \mathrm{m}$ hatch distance.

Figure 8-12: Scan strategy for single layer experimentation

112

Figure 8-13: Experimental setup for producing single layers of gold

Figure 8-14: Optical microscope image of single layer scan of gold: balling at $50 \mathrm{~W}$ laser power and $25 \mathrm{~mm} / \mathrm{s}$ scan speed.

Figure 8-15: Optical microscope image of single layer scan of gold: good sintering at $50 \mathrm{~W}$ laser power and $65 \mathrm{~mm} / \mathrm{s}$ scan speed

Figure 8-16: Optical microscope image of single layer scan of gold: unstable melt at $45 \mathrm{~W}$ laser power and $100 \mathrm{~mm} / \mathrm{s}$ scan speed

Figure 8-17: Microscope image of single layer scan of gold: weak sintering at $15 \mathrm{~W}$ laser power and $25 \mathrm{~mm} / \mathrm{s}$ scan speed.

Figure 8-18: Microscope image of single layer scan of gold: very little sintering at $10 \mathrm{~W}$ laser power and $25 \mathrm{~mm} / \mathrm{s}$ scan speed

Figure 8-19: Microscope image of first line balling at $50 \mathrm{~W}$ laser power and $65 \mathrm{~mm} / \mathrm{s} \mathrm{scan}$ speed 
Figure 8-20: (a) laser scanning first scan on the powder layer (b) laser scanning second scan on the powder layer with no powder available on one side 118

Figure 8-21: Smaller build platform with fill holes ........................................................ 119

Figure 8-22: Scanning Electron Microscope (SEM) image of gold cube........................ 120

Figure 9-1: Cross-sectional porosity variation with scan speed in gold cubes ...............124

Figure 9-2: Cross section image of sample produced with a scan speed of $25 \mathrm{~mm} / \mathrm{s}$ and

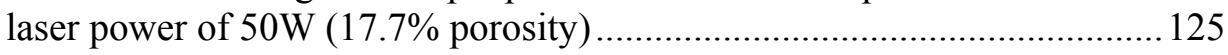

Figure 9-3: Cross section image of sample produced with a scan speed of $65 \mathrm{~mm} / \mathrm{s}$ and laser power of $50 \mathrm{~W}(12.5 \%$ porosity $)$

Figure 9-4: Cross section image of the sample produced with a scan speed of $100 \mathrm{~mm} / \mathrm{s}$ and laser power of $50 \mathrm{~W}$ ( $13.2 \%$ porosity) 126

Figure 9-5: Cross section image of the sample produced with a scan speed of $130 \mathrm{~mm} / \mathrm{s}$ and laser power of $50 \mathrm{~W}$ ( $14.4 \%$ porosity)

Figure 9-6: Multiple layer gold discs (a) bed temperature $28^{\circ} \mathrm{C}$ (room temperature) (b) bed temperature $100^{\circ} \mathrm{C}$

Figure 9-7: Porosity variation with layer thickness 129

Figure 9-8: Cross section image of the sample produced with a scan speed of $65 \mathrm{~mm} / \mathrm{s}$, laser power of $50 \mathrm{~W}$ and layer thickness of $50 \mu \mathrm{m}$ ( $7.7 \%$ porosity) ............ 129

Figure 9-9: Porosity variation with hatch distance ................................................... 130

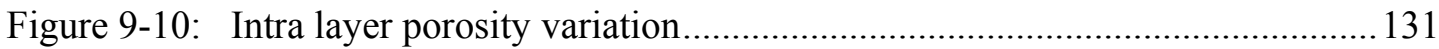

Figure 9-11: Cross section image of the sample produced with a scan speed of $65 \mathrm{~mm} / \mathrm{s}$, laser power of $50 \mathrm{~W}$ and layer thickness of $50 \mu \mathrm{m}$ ( $4.4 \%$ porosity) ............. 131

Figure 9-12: Thermocouple placed in the gold powder bed ........................................... 133

Figure 9-13: Schematics of the thermocouple incorporated into the gold powder bed for temperature measurements.

Figure 9-14: Temperature measured by thermocouple at different laser power.............. 134

Figure 9-15: Indentation in the gold cube during microhardness testing ........................ 135

Figure 9-16: Vickers Hardness (HV) for different gold samples ................................... 135

Figure 9-17: Load vs hardness for different hold time ................................................. 137

Figure 9-18: Hold time vs hardness for different loading rate ...................................... 137

Figure 9-19: Hold time vs modulus for gold cubes ..................................................... 138

Figure 9-20: Build orientation of (a) premolar and (b) molar dental parts ...................... 139

Figure 9-21: Gold premolar dental parts produced with $75 \mu \mathrm{m}$ and $50 \mu \mathrm{m}$ layer thickness

Figure 9-22: (a-c) Different views of gold premolar dental part ................................... 140

Figure 9-23: (a-c) Different views of gold molar dental part ......................................... 140

Figure 9-24: (a-d) Computerised Tomography (CT) scans at different sections of gold molar part

Figure 9-25: Optical image of the cross-section of gold premolar part........................... 143

Figure 9-26: (a-f) Cross-sectional images of gold premolar part at different locations .. 143 


\section{Abbreviations}

3DP

CAD

CNC

CW

CT

CAM

CWTD

CVTD

DRIFTS

DMD

DMLF

DMLS

DLF

EBM

LENS

LC

LBA

LSR

MRI

PSD

PET

RP

RM

RM

SLM

SLS

SFF

SLA

SEM

UV

UC

WEDM
3DPrinting

Computer Aided Design

Computer Numeric Control

Continuous Wave

Computer Tomography

Computer Aided Manufacturing

Constant Weight Tap Density

Constant Volume Tap Density

Diffuse Reflectance Infrared Fourier

Transform Spectroscopy

Direct Metal Deposition

Direct Metal Laser Fabrication

Direct Metal Laser Sintering

Direct Light Fabrication

Electron Beam Melting

Laser Engineered Net Shaping

Laser Consolidation

Laser Beam Analyzer

Laser Surface Remelting

Material Resonance Imaging

Particle Size Distribution

Positron Emission tomography

Rapid Prototyping

Rapid Manufacturing

Rapid Manufacturing

Selective Laser Melting

Selective Laser Sintering

Solid Freeform Fabrication

Stereo lithography

Scanning Electron Microscopy

Ultra Violet

Ultrasonic Consolidation

Wire Electric Discharge Machine 


\section{Nomenclature}

\begin{tabular}{|c|c|}
\hline$\Delta H_{m}$ & Energy required to melt $[\mathrm{J}]$ \\
\hline$\rho$ & Density of the material $\left[\mathrm{kg} / \mathrm{m}^{3}\right]$ \\
\hline$v_{p}$ & Volume of the particles $\left[\mathrm{m}^{3}\right]$ \\
\hline$C_{p}$ & Specific heat capacity $[\mathrm{J} / \mathrm{kg} \mathrm{K}]$ \\
\hline$\Delta T_{m}$ & $\begin{array}{l}\text { Temperature difference between ambient and material melting } \\
\text { temperatures }\left[{ }^{\circ} \mathrm{C}\right]\end{array}$ \\
\hline$L_{f}$ & Latent heat of fusion $[\mathrm{J} / \mathrm{kg}]$ \\
\hline$E_{m}$ & Melt energy $[\mathrm{J}]$ \\
\hline$r_{p}$ & Particle radius [m] \\
\hline$\gamma_{S V}$ & Surface tension of solid in air $[\mathrm{N} / \mathrm{m}]$ \\
\hline$\gamma$ & Interfacial Energy $[\mathrm{N} / \mathrm{m}]$ \\
\hline$\gamma_{L S}$ & Interfacial surface tension of liquid and solid [N/m] \\
\hline$\gamma_{L V}$ & Surface tension of liquid in air $[\mathrm{N} / \mathrm{m}]$ \\
\hline $\mathbf{L}$ & Melt pool length [m] \\
\hline $\mathbf{D}$ & Melt pool diameter $[\mathrm{m}]$ \\
\hline $\boldsymbol{\theta}$ & Contact angle [degree] \\
\hline$M_{a}$ & Marangoni number \\
\hline$\eta$ & Viscosity $[\mathrm{Pa} \mathrm{s}]$ \\
\hline $\boldsymbol{\delta}$ & Thermal diffusivity $\left[\mathrm{m}^{2} / \mathrm{s}\right]$ \\
\hline$I_{o}$ & Incident radiation intensity \\
\hline$\Delta \mathbf{G}$ & Free energy of the reaction $[\mathrm{J} / \mathrm{mol}]$ \\
\hline $\mathbf{R}$ & Universal gas constant $[\mathrm{J} / \mathrm{mol} \mathrm{K}]$ \\
\hline $\mathbf{T}_{\mathbf{a b s}}$ & Absolute temperature $\left[{ }^{\circ} \mathrm{C}\right]$ \\
\hline $\mathbf{P}_{\mathrm{O} 2}$ & Partial pressure of oxygen \\
\hline $\mathbf{R}_{\mathbf{m}}$ & Reflectance \\
\hline$A$ & Absorbance \\
\hline$T$ & Transmittance \\
\hline $\mathbf{K}_{\mathbf{a b s}}$ & Absorption coefficient $[\%]$ \\
\hline $\mathbf{T}$ & Time $[\mathrm{s}]$ \\
\hline $\mathbf{I}_{\mathbf{r}}$ & Reflected radiation intensity \\
\hline $\boldsymbol{\rho}_{\mathbf{a}}$ & Apparent density $\left[\mathrm{g} / \mathrm{cm}^{3}\right]$ \\
\hline $\mathbf{W}$ & Weight of the material $[\mathrm{g}]$ \\
\hline
\end{tabular}




$\begin{array}{ll}\mathbf{p t}_{\mathbf{n}} & \text { Tap density after 'n' number of taps }\left[\mathrm{g} / \mathrm{cm}^{3}\right] \\ \mathbf{V}_{\mathbf{n}} & \text { Volume occupied by material after ' } \mathrm{n} \text { ' number of taps }\left[\mathrm{cm}^{3}\right] \\ \mathbf{p}_{\mathbf{t}} & \text { Tap density }\left[\mathrm{g} / \mathrm{cm}^{3}\right] \\ \mathbf{p}_{\mathbf{b}} & \text { Aerated or apparent density }\left[\mathrm{g} / \mathrm{cm}^{3}\right] \\ \mathbf{d}_{\mathbf{h}} & \text { Hatch distance }[\mu \mathrm{m}] \\ \mathbf{r} & \text { Laser spot radius }[\mu \mathrm{m}] \\ \mathbf{A} & \text { Area }\left[\mu \mathrm{m}^{2}\right] \\ \boldsymbol{v}_{\text {scan }} & \text { Laser scan speed }[\mathrm{mm} / \mathrm{s}] \\ \mathbf{P} & \text { Laser power }[\mathrm{W}] \\ \mathbf{T}_{\text {layer }} & \text { Layer thickness }[\mu \mathrm{m}] \\ \mathbf{H V} & \text { Vickers hardness number }\end{array}$




\section{Chapter 1}

\section{INTRODUCTION}

\subsection{Scope of Research}

The Selective Laser Melting (SLM) process is capable of producing complex 3D metallic parts that would be otherwise difficult to produce using conventional manufacturing processes like casting and machining etc. In the SLM process the metal powder is completely melted and re-solidified which enables it to create parts with high density and strength as compared to other laser based processes like Selective Laser Sintering (SLS). The complete melting and solidification of metal in the SLM process induces problems like balling, marangoni flows and solidification cracking. Before a material is qualified to be processed using SLM, suitable processing parameters are identified so that the afore mentioned problems are minimized.

SLM has been used in the medical and dental field for manufacturing prostheses and dental implants. The materials used for dental purposes include biocompatible metals, ceramics and composite of them. Porcelain-fused-to-metal is currently being used for dental restorations. The substructure in the porcelain-fused-to-metal restorations is made from biocompatible metal and coated with the hard porcelain to give it the desired strength and aesthetics. The metal substructure is made by the lost wax casting method. The lost wax casting process for manufacturing dental crowns and bridges involves many pre and post processing steps. Pre-processing steps involve taking impressions of the patient's teeth, making a plaster model and then the wax model before the casting process. Postprocessing steps include the delicate process of removing the casting from the mould (as the parts have thin walls and fine features), detaching it from the casting tree and manual finishing the part. The SLM process could be used to manufacture these complex substructures for porcelain-fused-to-metal restorations from metals with ease and fewer processing steps. Precious metal and alloys (especially gold and gold alloys) have been used for manufacturing dental crowns and bridges for centuries. The use of high caratage gold alloy enables good shades for porcelain-fused-to-metal restorations (Cascone, 2003) but due to the casting problems associated with pure gold, it is not used for dental purposes. ARGEN Corporation USA developed a unique 24 carat castable gold alloy 
which consists of $99.7 \%$ gold and traces of zinc $(0.1 \%)$ and indium $(0.2 \%)$. This alloy is presently cast to produce dental crown substructures for porcelain fused to metal restorations (Cascone, 2003). The SLM process has been shown to be feasible to process a variety of metals and alloys and SLM could be used to process precious metals and alloys for producing dental crowns and bridges. This research work focuses on the feasibility of SLM to process 24 carat gold powder and its possible application in manufacturing metal substructures for porcelain-fused-to-metal dental crowns. Figure 1-1 shows the present research work in the overall context of Rapid Manufacturing (RM).

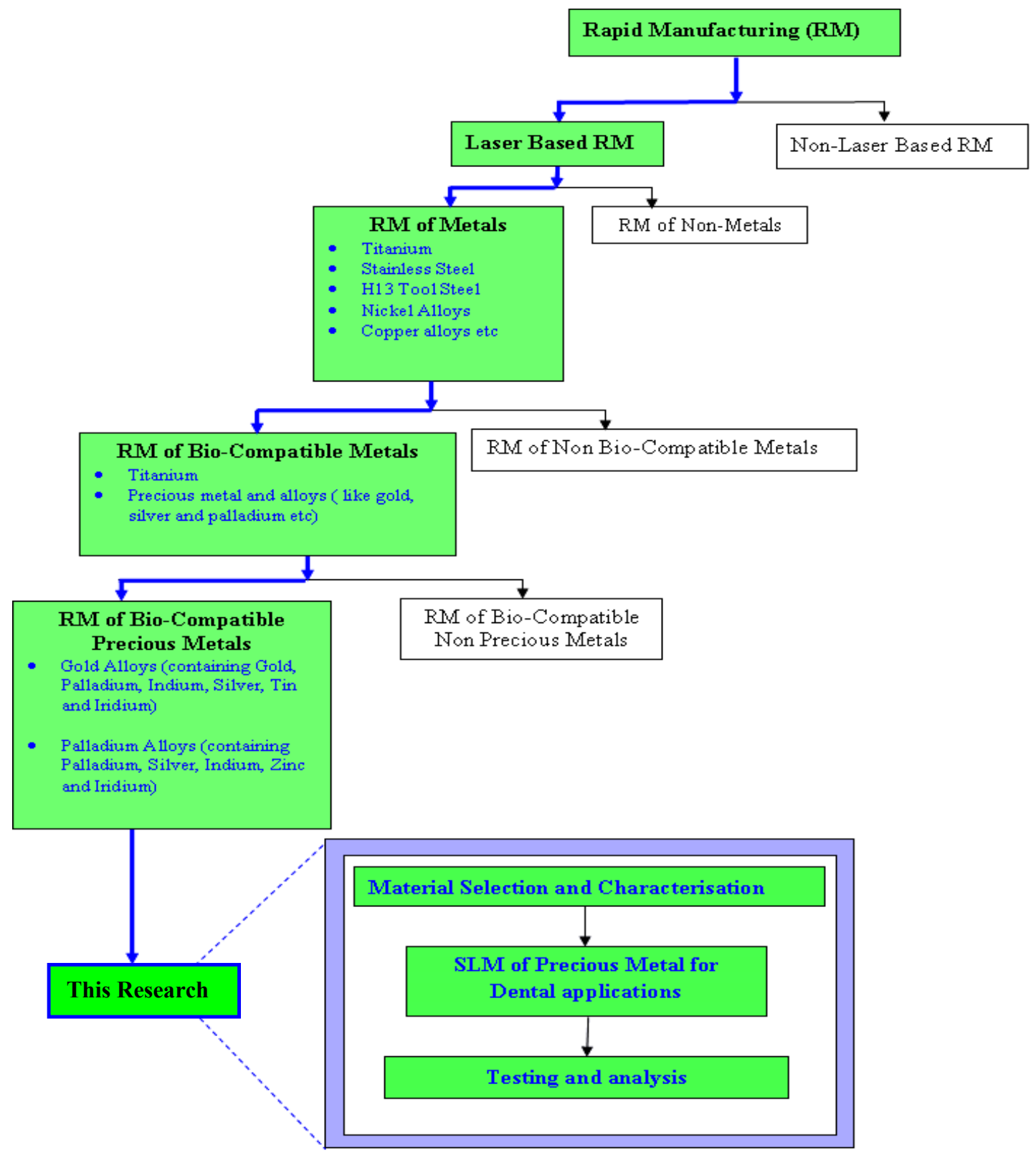

Figure 1-1: Flow chart explaining task of this $\mathrm{PhD}$ research 


\subsection{Thesis Outline}

Chapter 2 explains different Solid Freeform Fabrication (SFF) processes, techniques and the available technologies. The SLM process is also explained in detail including the factors affecting processing of metals and the thermodynamics aspect of the SLM process. Chapter 3 details the application of SFF and specifically SLM in the medical and dental fields. Chapter 4 defines the novelty and objectives of this research work Chapter 5 focuses on the radiation absorption behaviour of different metals such copper, stainless steel, H13 tool steel and gold powder etc. The effect of powder bed compaction and particle size on the absorption properties of the powder is also explained. Chapter 6 discusses the powder properties including particle size distribution, apparent density, tap density and moisture absorption. Chapter 7 summarizes different aspects of the processing system and the modifications made to the existing system for gold powder deposition and processing. Chapter 8 details the SLM of 24 carat gold powder and the related issues such as balling etc. This chapter ends with the manufacturing of gold cubes. Chapter 9 discusses the identification of optimum parameters, internal porosity of gold cubes, its mechanical properties, the effect of powder bed temperature and the manufacturing of complex gold substructures for dental crowns from 24 carat gold powder.

Chapter 10 summarizes the conclusions drawn form this research work and suggests the future work. 


\section{Chapter 2}

\section{BACKGROUND}

\subsection{Solid Freeform Fabrication (SFF)}

Solid Freeform Fabrication is a group of non-conventional manufacturing technologies comprising of additive manufacturing processes to produce a complex 3D object in a digital, highly flexible and efficient environment.

SFF technologies can be divided into different categories according to the actual process of manufacturing, materials used, material feed method and type of energy input. Figure 2-1 shows the sectors that are using SFF technologies, the major sector being consumer products and electronics. Due to the high flexibility provided by these technologies, more and more industries are taking interesting them. Figure 2-2 shows the broader classification of some of the SFF technologies

Figure 2-1: Different sectors embracing SFF technologies (Wohlers, 2008) 


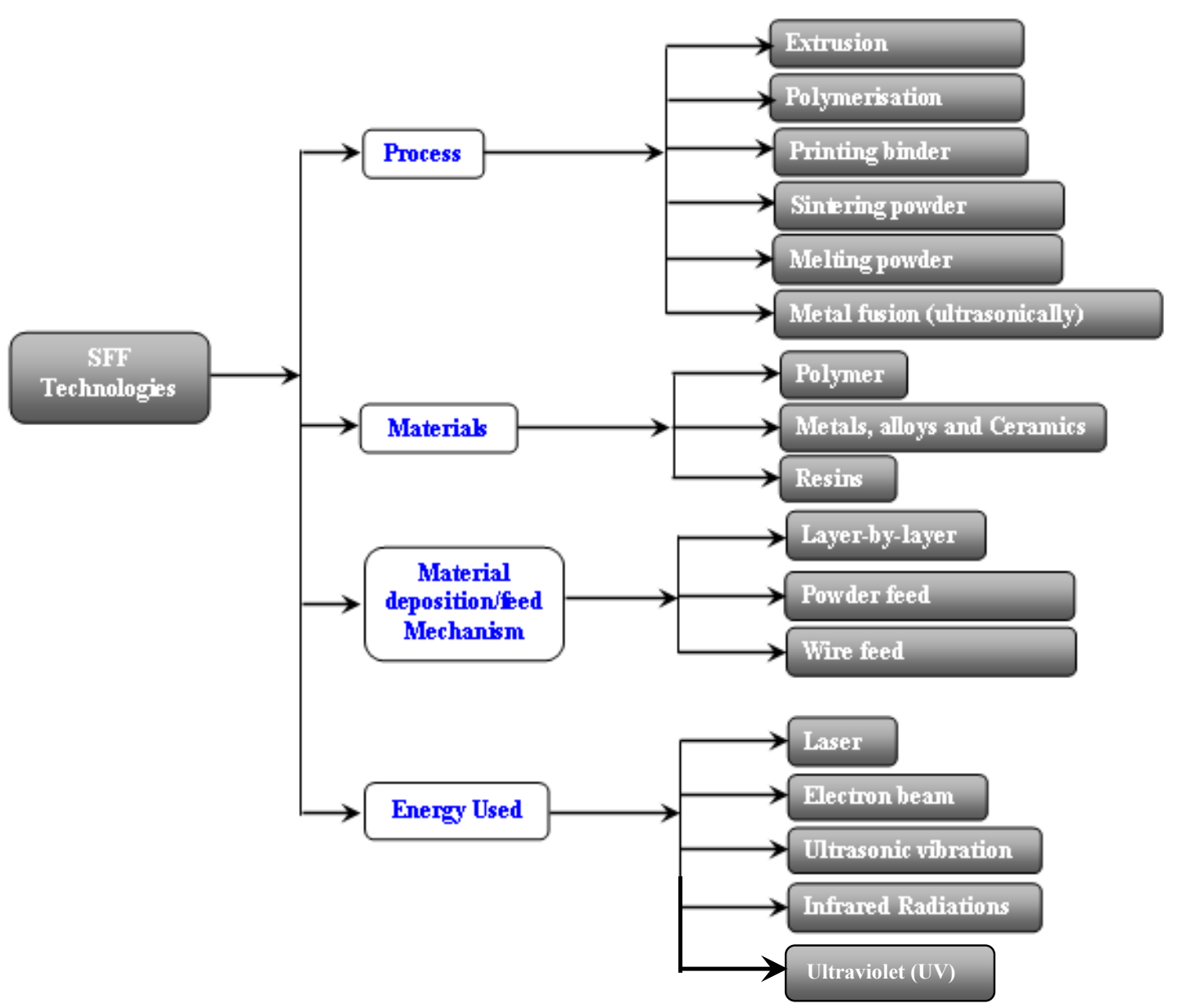

Figure 2-2: Classification of SFF technologies 


\subsection{Solid Freeform Fabrication (SFF) of Metals}

Different types of materials such as polymers, metals, ceramic and composites are presently being processed by SFF technologies. However, manufacturing of metallic parts which can be directly used in different applications, is the focus of major research presently. Typical standard metals which have been widely processed by SFF technologies are stainless steel (Over et al, 2001) and tool steel powders (Akhtar et al, 2003) for tooling and parts manufacturing. In recent years, non-ferrous powder materials are gaining a lot of interest because of their applications in sectors like medical and aerospace. Using this technology, parts have been manufactured from pure titanium (Abe et al, 2000), titanium alloys like Ti6Al-4V (Pohl et al, 2004), cobalt-chromium alloys (Strietzel, 2004), Nickel alloys (Mumtaz, 2008) and aluminium alloys (Edelmann and Mueller-Lohmeier, 2005) to name a few. Recently, SFF technologies have also been used to process different materials in the category of bio-compatible and high performance alloys. The SFF technologies that process metals can be divided into two broad categories based on the type of energy they use i.e. non-laser based and laser based technologies.

Laser based SFF processes are also known as Direct Metal Laser Fabrication (DMLF). Some DMLF technologies include Selective Laser Melting (SLM), Direct Metal Laser Sintering (DMLS), Laser Cusing, Laser Engineered Net Shaping (LENS), Direct Light Fabrication (DLF), Direct Metal Deposition (DMD), Laser Consolidation (LC) and Deposit Laser Welding.

Some non-laser based processes include Electron Beam Melting (EBM), Ultrasonic Consolidation (UC), Maskless Mesoscale Material Deposition (M3D), Shape Deposition Manufacturing (SDM), Cold Gas Dynamic Manufacturing (CGDM), Layered Object Manufacturing (LOM), 3D Printing (3DP).

\subsubsection{Key Non-Laser Metal Processing Technologies}

Key non-laser based metal processing technologies are discussed in detail below: 


\subsubsection{Electron Beam Melting (EBM)}

The EBM process uses electrons (as in the Electron Beam Welding (EBW) process) to completely melt the pre-spread powder layer for creating 3D parts. EBM has been developed by Arcam. The EBM process is capable of creating high density metallic parts at higher build speeds due to the high traversing speeds of the electron beam (Cormier et al, 2004) (Williams et al, 2005). A disadvantage of the EBM is the rough surface finish of the parts which usually requires post processing.

\subsubsection{3D Printing (3DP)}

The 3D printing process involves binding the pre-spread metal powder with a polymer to create green parts. These green parts are then further processed in a furnace to sinter the powder followed by infiltration to create strong 3D objects (Feensta et al, 2003) (Williams et al, 2005). Similar to EBM, this process also yields high surface roughness and involves further post processing to improve part quality. Prometal is the license holder of this technology.

\subsubsection{Ultrasonic Consolidation (UC)}

The ultrasonic consolidation process uses ultrasonic vibrations with an applied load to join metal foils to create 3D objects (Kong et al, 2003). The UC process is very flexible in respect to embedding different elements inside the component but it is very difficult to produce overhanging structures with this process.

\subsubsection{Laser Processing of Metals}

Different metals including high performance and bio-compatible metals are currently being processed and optimized for different engineering applications. Gu and Shen (2007) processed copper powder to investigate the reasons behind the balling phenomenon (discussed in detail in section 2.3.3.3) in the Direct Metal Laser Sintering (DMLS) process, Mumtaz et al (2008) processed nickel based super alloy (Waspaloy®) using a high power pulse Nd:YAG laser system, Yadroitsev et al (2007) processed Inconel 625 using Phenix System's PM 100 system, Simchi (2006) sintered various ferrous powders: 316L stainless steel powder and M2 powders with an EOS M250X ${ }^{\text {tend }}$ machine, Rombouts et al (2006) investigated laser melting of

different sizes and mixture of water and gas atomized steel powders, Simchi (2004) also investigated the particle size effect on the sintering of iron powders, Fischer et al (2003) sintered commercially pure titanium powder using an Nd:YAG laser. All these materials find 
their use in different fields. Titanium, cobalt chrome and some precious metal alloys have been used in manufacturing medical implants because of their bio-compatibility. Nickel based super alloys, Inconel 635, 316L stainless steel and M2 alloys have been used for high performance applications such as in aerospace. Figure 2-3 and Figure 2-4 show components manufactured from stainless steel and aluminium 6061. These samples were prepared in a Phenix Systems PM-100 machine and MCP Realizer II respectively.

Figure 2-3: Lattice spatial structure fabricated from stainless steel grade 316L (Yadroitsev et al, 2009)

Figure 2-4: $\quad$ SLM fabricated heat sinks, base dimensions $50 \mathrm{~mm} \times 100 \mathrm{~mm}$, flow in the $\mathrm{z}$ direction. (a) Pin fin 6061, (b) Rectangle, (c) Rect RND, (d) Ellipse, (e) Lattice and (f) close up of Lattice. 
Although metallic parts have been manufactured by SFF technologies, they are currently facing issues of quality, repeatability and standardization (to be qualified to be directly used in critical applications like the aerospace industry). Many people from the field of academia and industry are currently focusing on these issues and it is the focus of much current research.

\subsubsection{Sintering mechanisms}

The amount of energy delivered to the powder material determines the degree of particle melt and eventually the quality of the final part. Sintering can be differentiated broadly into three categories namely Solid State Sintering, Liquid Phase Sintering or Partial Melting and Complete Melting. These are explained in detail below.

\section{Solid State Sintering (SSS)}

Solid state sintering is the thermal process which occurs at a temperature range between $T_{\text {melt }} / 2$ to $T_{\text {melt }}$, where $T_{\text {melt }}$ is the melting temperature of the material being processed (Kruth et al, 2005). Between this temperature range, apart from other physical and chemical changes that take place, the important phenomenon of diffusion also occurs (Van Der Schueren, 1996). The binding of powders through diffusion does not involve any liquid phase; rather necks are formed between the two particles in contact as shown in Figure 2-5.

Figure 2-5: (Left) Neck formation in SSS, (Right) Neck formation between two stainless steel particles (Van Der Schueren, 1996)

Diffusion involves the formation of necking between the powder particles. The National Academy of Science of Belarus, Baikov Institute of Metallurgy and University of Leuven investigated the SSS of titanium powder using a low intensity laser light (100- 
$300 \mathrm{~W} / \mathrm{cm}^{2}$ ) for 5 seconds. The temperature of the process was kept below the melting temperature of titanium which resulted in necking between titanium particles. The degree of sintering of these particles was quantified by the ratio of neck diameter to the particle diameter $(\mathrm{D} / 2 \mathrm{R})$. Because of the slow nature of the process of diffusion in SSS, the build time is very high and thus this process is very rarely used (Kruth et al, 2007).

\section{Liquid Phase Sintering (LPS)/Partial Melting}

LPS can be achieved by different methods, most common being that the base material remains solid throughout the process while the other material melts and binds the particles of the base material. The material that binds the particles is called the binder. In some cases, the solid and liquid material is the same and these are differentiated by the melted and non melted phases (Kruth et al, 2005). In the LPS category, there is limited movement of the solid particles with the capillary forces, which, in most cases results in porosity (Rombouts, 2006). In partial melting no binding material is used rather the particle's outer surface is melted while its core is still solid. In this case the energy supplied to these particles is not enough to completely melt them (Kruth et al, 2007). Like SSS the parts produced with LPS or partial melting usually require some post processing to increase their strength and structural integrity as shown in Figure 2-6.

Figure 2-6: Liquid Phase Sintering (LPS) of WC-Co powder mixture, left: before infiltration (a: non-molten WC particle, b: molten Co, c: porosity), right: after infiltration with copper (Kruth et al, 2005)

In the SLS process, metal powder is mixed with a plastic powder which acts as a binder for the metal particles. Laser scanning over the powder bed heats and melts the plastic which keeps the metal particles embedded in it to form a shape of the final part. The part at 
this stage is called a 'green part'. This part is then processed in a furnace where the binder is removed and the metal particles sinter together to form a solid part. However, this part is still only $60 \%$ dense and needs infiltration by another metal to fill the pores in it. The infiltration can be done using other metals such as bronze in the furnace (Rossi et al, 2004).

\section{Complete Melting}

In this category of sintering, enough energy is provided to the powder material to completely melt it. The complete melting of metal powder requires high laser energy and can produce parts with almost $100 \%$ density in a single step. Figure 2-7 shows titanium processed by the Selective Laser Melting (SLM) process. It can be seen that the part is almost fully dense except for a crack due to thermal stresses. Thermal stresses in the final part is one of the issues with complete melting and is discussed in detail in section 2.3.3.7.

Figure 2-7: Cross section of a Ti part produced by Selective Laser Melting (Kruth et al, 2005)

\subsection{Selective Laser Melting (SLM)}

SLM is one of the key DMLF processes. In SLM, metal particles are completely melted to form a solid part and no further post processing is required in the furnace. Different process parameters are adjusted to get fully dense, direct to use metal parts. In theory, all metals or metal alloys could be processed by SLM; however, the range of commercially available metals for SLM is still limited today. This is because the ease of processing differs a lot due to the physical properties of the materials e.g. thermal expansion coefficient, melting point, thermal conductivity, surface tension, laser absorption and viscosity etc. 
This section explains the SLM process, effect of different factors on the process and its thermodynamics.

\subsubsection{The SLM Process}

The typical configuration of an SLM machine is shown in Figure 2-8. The parts are manufactured on the base plate (also called substrate plate) which is mounted on a piston that moves vertically in the build cylinder. A container stores the powder material which is spread on top of the base plate by different means such as roller, scraper or a cantilever hopper in this case. A laser scans a single cross-section of the Computer Aided Design (CAD) model on the power layer thus melting the powder to make a solid part. The vertical motion steps of the piston are equivalent to the thickness of each slice of the CAD model. The laser scanning assembly incorporates a mirror system to scan at different locations on the base plate. The FTheta lens focuses the laser and provides a flat image field along the plane of interest. When the laser strikes the powder particles, energy is absorbed from the laser which heats and eventually melts the powder (depending on the amount of energy absorbed) to form a solid object. This process is repeated for each slice of the CAD model to make a complete 3D object. The process is carried out in an inert environment to reduce oxidation of the metal. The powder bed preheating is carried out using the in built heaters in the platform. 


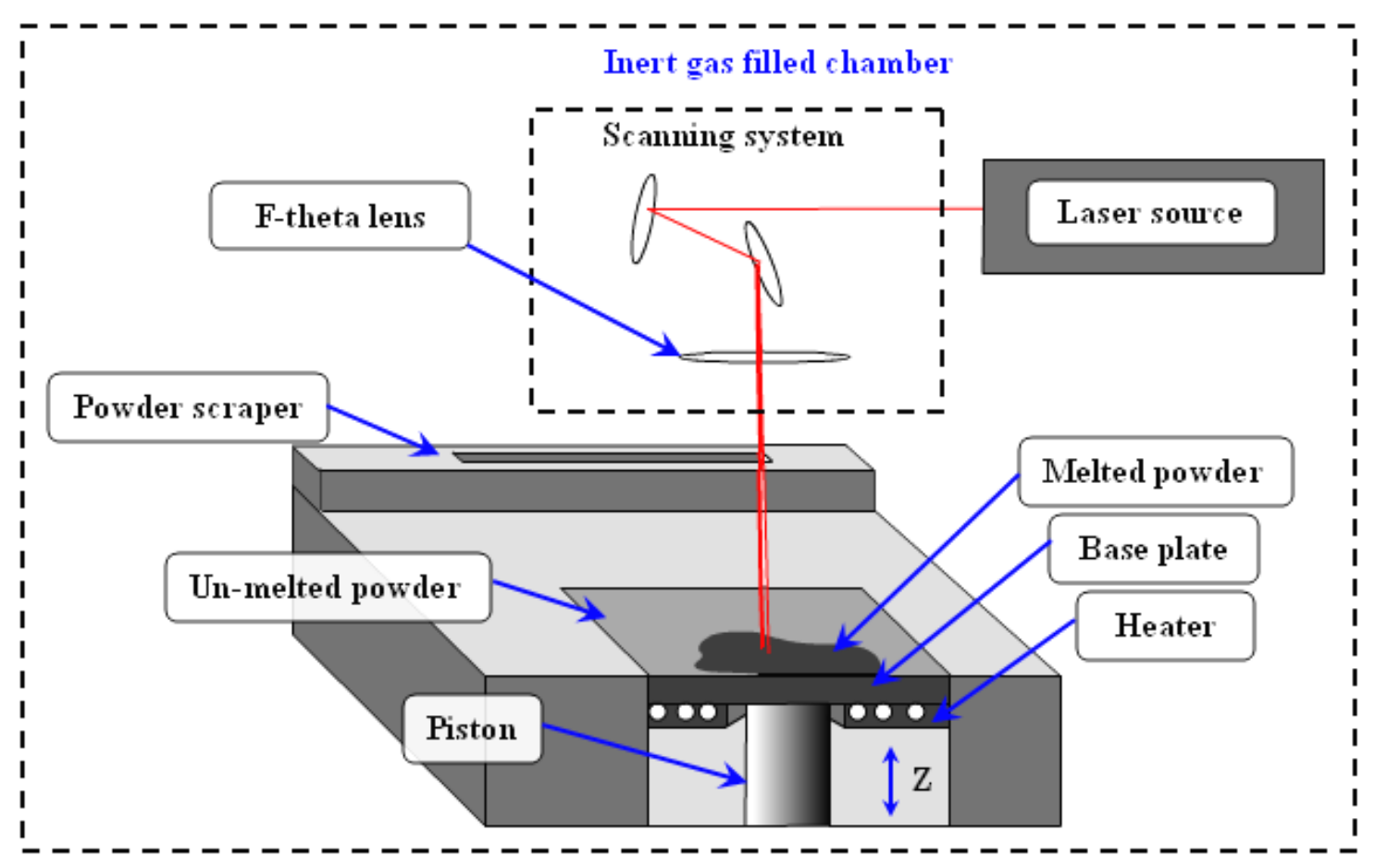

Figure 2-8: $\quad$ Schematics of the SLM process

\subsubsection{Factors Affecting the SLM Process}

The SLM process parameters can be divided into four main groups: material, laser, scan and environment. This section gives a brief description of these parameters.

\subsubsection{Material}

Material properties for a specific application are constrained by the application requirements. Once a material is selected for processing by SLM, material characterization is performed to check its physical properties and chemical composition. Physical properties like density, thermal capacity (specific heat capacity), latent heat of fusion and melting temperature determine the melt enthalpy of the metal. The melt enthalpy is the amount of energy needed to melt the powder completely and it determines the heat balance (Van Elsen, 2007). The thermal conductivity is the main driving factor in the heat balance and the thermal expansion coefficient determines the stresses introduced during re-solidification of the molten metal. These material properties are determined after the material selection phase. The chemical composition of the metal defines its alloying behaviour in a multi component material which affects shrinkage and wettability etc. 
Absorption of incident radiation by a powder bed is quite different from bulk metal (Tolochko et al, 1997). Multiple interactions of the incident radiations in the small pores present in the powder bed increase its laser absorption. Powder absorption depends on compaction, (nature of) material (intrinsic material absorption characteristic i.e. copper is more reflective than stainless steel) and wavelength of incident radiations. This is explained in more detail in Chapter 5 .

A majority of the SFF technologies use metal in powder form. Some of the properties associated with the powder material are detailed below:

\section{Particle Size Distribution (PSD)}

Particle Size Distribution (PSD) shows the frequency of a particle size in a sample of powder material. PSD affects the important powder characteristics of flowability and compaction (German, 1989). A wider PSD is known to increase the powder bed density of the material, as wider PSD ensures that there is enough smaller particles to fill the gaps between larger particles in the powder bed. This high compaction of the powder bed in turn increases the sintered density of the material (Zhu et al, 2007). The ratio of larger to smaller particles in the powder can influence the flowability of the particles in layer based SFF processes (Other factors such as humidity and shape of the particles can also influence flowability). If the powder contains a large number of smaller particles, the chances of agglomeration are increased. This is due to the Van der Wall forces being more pronounced in smaller particles (small particles have high surface area to volume ratio) thus making the deposition process more difficult (Simchi, 2004) (Boivie, 2001). Packing density and flowability of the powder is further discussed in detail in Chapter 6. On the other hand, a very narrow PSD can improve the consistency of the melt but reduces the packing density of the material (Lang et al, 2000). Apart from this, PSD can also influence the part quality. A greater number of smaller particles reduces the energy required to melt them and also improves the part surface roughness (Karapatis et al, 1998) (Sears, 1999) (Syvanen et al, 2000) (Lu et al, 2001). If the powder consists of smaller particles, thinner layers can be deposited (depends on the flowability of the powder). According to Mazumder et al (2000), with thicker layers, the laser beam has a larger distance to diverge and forms a melt pool which is larger at the bottom as compared to the top. 
By reducing the particle size, the layer thickness can be reduced, thus reducing the side surface roughness of the part.

Based on the above discussion, selection of a specific PSD powder is very important to the process and final quality of the parts. To some extent there is a compromise between the percentage of smaller and larger particles. If a lot of smaller particles are selected, the surface roughness is reduced and so is the layer thickness thus improving the part quality, but there is more chance of agglomeration and problems in the deposition process. If the powder consists of a very high percentage of larger particles, the flowability is improved but the powder bed density is reduced which could reduce the final density of the part. Therefore, a powder should consist of adequate amount of smaller particles to fill the gaps and increase the powder bed density but not influence the powder flowability and deposition (Zhu et al, 2007).

\section{Particle morphology}

Powder morphology is the shape of the particles in the powder. The powder morphology is directly influenced by the preparation method i.e. milled, water atomized or gas atomized. The gas atomization technique is found to produce more spherical particles as compared to the other two. Spherical particles are known to improve powder flowability and the quality of the layer and final product in SFF technologies (Niu and Chang, 1999). A nonspherical powder has been observed to have less compaction and hence increased porosity in the parts.

\section{Density}

Densities of powder are of two types i.e. individual particle density and the packing density (apparent and tap densities) of the powder as a whole. The individual particle density depends upon the type of material and is an intrinsic material property, while the packing density can be a loosely packed density or tapped (compacted) density of the powder depending on the compaction method.

\section{Thermal conductivity}

The thermal conductivity is the material's ability to conduct heat across individual particle(s) and changes with the temperature of the particles. The thermal conductivity of the

powder bed (or layer of powder) with a certain thickness depends on the number of contacts of 
the particles, which in turn depends upon the compaction of the powder. The higher the compaction the more will be the contact points and thus higher heat transfer will take place across the powder layer.

\section{Specific heat capacity}

Specific heat capacity or specific heat is the amount of heat energy required to raise a unit quantity of material by one degree in temperature. The specific heat capacity of the material affects the heat balance (see section 2.3.3.3).

\section{Latent heat of fusion}

The latent heat of fusion is defined as the amount of energy required to change the state of unit mass of material from solid to liquid without rise in temperature. It is also used in heat balance (see section 2.3.3.3)

\section{Melting point}

The melting point of a material is the temperature at which the material in solid state will change into liquid phase.

\section{Evaporation point}

Similar to melting point, the evaporation point is the temperature at which the material will change its phase from liquid to gas.

\section{Viscosity}

Viscosity is resistance of the material to flow in the molten state. The viscosity of the material in molten state changes with temperature and influences the part quality (discussed in detail in section 2.3.3.3)

\section{Surface free energy}

The surface free energy quantifies the disruption of chemical bonds when a surface is transformed/ changed (Van Elsen, 2007) the surface free energies have effect on the wetting and balling behaviour of the molten material (Kruth et al, 2003). Both of these phenomena are explained in detail in section 2.3.3.3. 


\section{Absorption (percentage)}

The absorption is the percentage of the incident radiation which is absorbed by a material. It is also known as laser/energy coupling (further discussed in section 2.2.3.1)

\subsubsection{Laser}

Since SLM process melts the powder by thermal energy of a laser beam, properties of the laser beam like spot size, wavelength, laser power and the form in which the energy is delivered (pulsed or continuous) are extremely important. The laser spot size is usually adjusted before building the part and is not changed during the operation. Many laser parameters are fixed by the system, e.g. wave length, mode, intensity profile, beam quality and minimum spot size etc. In practice, the only laser parameters that can be varied are laser power and spot size for continuous laser, where a larger spot size could be adjusted to an out of focus position for laser surface re-melting applications (LSR) (Deckers, 2008). For pulsed laser the adjustable parameters are pulse frequency, spot size, pulse duration and peak power. These two laser types are explained in detail below:

\section{Pulse and Continuous Wave Laser}

A pulse laser uses small burst(s) of energy to melt the material and the melt pool generated is kept molten by repeated pulses (Yevko et al, 1998). A pulsed laser scans a straight line by multiple overlapping spots of these molten zones which are also evident in the overlapped solidified material. The pulse laser system generally has more parameters to control such as pulse energy, pulse duration, repetition rate (which allows for a wider range of experimental conditions) and energy control. This helps in fine tuning the experimental parameters for better results. Pulse shaping is also known to effect the properties of the built part, where a ramp-up pulse has a positive affect on reduction of part distortion and residual stresses, and ramp-down increases the time for which the material is kept in the molten state and helps improve top surface roughness (Mumtaz, 2008).

A continuous laser is generated by a continuous excitation of the reactive medium and the energy output is almost constant. Continuous lasers are known to heat and melt the powder homogeneously (Fischer et al, 2002). Due to a limited number of parameters to be adjusted (laser power, scan speed and spot size), the part production has fewer variables with a continuous laser system as compared to a pulse laser system. Newer commercial SLM systems 
incorporate continuous wave lasers operated in the pulse laser fashion. Instead of laser energy emitted in a continuous fashion, the laser energy is emitted in short bursts similar to a pulsed laser. Thus the laser operates in a pulse like mode but with constant energy with respect to time; whereas in the pulse mode, the pulse shape has an effect on the heat build-up in the material over time. This method of laser energy delivery is carried out such that heat build up and melt pool width is minimised, reducing the size of thin features created by these systems and could also help in eliminating balling.

\section{Laser Wave length}

Direct metal fabrication of metals involves the use of different laser source(s). Two of the most extensively used laser source(s) for processing metals are Carbon Dioxide $\left(\mathrm{CO}_{2}\right)$ lasers and Neodymium-Doped Yttrium Aluminium Garnet (Nd:YAG) lasers. Currently a new type of solid state laser called Ytterbium fibre laser is being increasingly used for processing metals. The fibre laser uses a fibre doped with the active gain medium. The operating wavelength of the $\mathrm{CO}_{2}$ laser is $10.6 \mu \mathrm{m}, \mathrm{Nd}: Y A G$ laser is $1.06 \mu \mathrm{m}$ and for fibre laser it is 1.07$1.09 \mu \mathrm{m}$ (IPG photonics YLR-50). It is known that metals have higher reflectivity at longer wavelength of the incident laser (Ready, 2001). Therefore, at shorter wavelength the metal will absorb a higher percentage of the incident radiation thus making the processing easier (Laeng et al, 2000). As the Nd:YAG laser and fibre laser operate at relatively similar wavelengths, it is easier to process metal with them as compared to the $\mathrm{CO}_{2}$ laser. Of all these lasers, a fibre laser has the highest beam quality because of the wave guiding property of the fibre eliminating thermal distortion along the path (Photonics, 2009). According to Kruth et al (2003), most metals will have $20-30 \%$ reflectance to the Nd:YAG and fibre laser wavelengths. Presently, the majority of commercial systems (EOS M270, MTT Realizer, Concept Laser's LaserCusing etc.) incorporate a fibre laser which can be used in continuous and Q-switched modes (generating nanosecond pulses).

Absorption of radiation by a metal also depends on different factors such as type of surface (powder bed or solid surface), powder bed compaction, type of material and temperature which are explained in detail in Chapter 5. 


\subsubsection{Environment}

The environmental factors which have an influence on the SLM process include the type of gas filled in the chamber, gas pressure and flow and powder bed pre-heating etc. All these factors are discussed in detail in section 2.3.3.6.

\subsubsection{Thermodynamics of the SLM Process}

This section describes in detail the thermodynamic aspect of the SLM process i.e. laser material interaction, heat transfer in the powder bed, melt pool dynamics, environmental and thermal effects on the final part.

\subsubsection{Laser Material Interaction}

Laser material interaction or laser material coupling is a complex phenomenon that depends upon the absorption of incident radiation by the material. Different factors affect the absorption of radiation by a material such as direction of incident radiations, surface roughness, surface oxides, wavelength of the incident radiation, temperature of the material and type of material.

\section{Absorption}

In metals, the radiation is predominantly absorbed by the free electrons. The incident radiation is absorbed, reflected or transmitted through the material. The incident radiation is only absorbed to a certain depth in the materials (called the optical penetration depth) which is up to a couple of atomic diameters in metals (Steen, 2003), therefore the transmitted portion is negligible (Karapatis, 2002).

The absorption of radiation by a material is a surface phenomenon and the surface oxide film, if any, would act as an anti-reflective coating for the material underneath. The surface oxide films needs to be of a certain thickness to have a considerable effect (Steen, 2003).

The surface roughness of the material being irradiated also has an effect on the absorption by that surface. Ang et al (1997) showed that a rougher surface increases 
absorption of the incident radiation. Also, the absorption is low for radiation incident perpendicular to the material surface.

The wavelength of the incident radiation also has a pronounced effect on the absorption of radiation by the target material. At longer wavelengths, metals have lower absorptivity (higher reflectivity) and vice-versa. At shorter wavelengths, the higher energy photons from the radiation can be absorbed by the electrons in the material, resulting in higher absorption (Steen, 2003).

Absorption of the material is also temperature dependent and it tends to increase with increasing temperature. As the temperature of the material is increased, the phonon population in the material increases. A Phonon is a quantized mode of vibration in the rigid crystal lattice of the material. As the phonons increase in number, there is a greater phonon-electron energy exchange which increases the interaction of free electrons with the structure of the material rather than vibrating and re-radiating some energy. Thus a higher percentage of the incident radiation is absorbed by the material at higher temperatures (Ready, 2001)

The radiation absorption of a flat polished material surface is different from a flat powder layer because of the surface roughness and micro gaps in the packed powder layer. As most of the RM technologies utilise powdered material, the laser absorption values of bulk materials cannot be used. In RM the laser energy is absorbed by the first layer of particles exposed to this radiation. These particles transfer this energy through conduction, convection and radiation, where the radiation contribution is almost negligible. The heat generated by the laser will travel through the surrounding powder particles via conduction and if sufficient, will completely liquefy powder particles forming a melt pool (Mumtaz, 2008). It is also necessary to understand the radiation absorption of a material in powder form, especially highly reflective metal powders like gold, silver and aluminium etc. to identify the wavelength range in which the absorption of these powders is high. This will facilitate selecting the right processing laser source $\left(\mathrm{CO}_{2}\right.$ laser with $10.6 \mu \mathrm{m}$ wavelength or fibre laser with $1.06 \sim 1.09$ $\mu \mathrm{m})$ for these materials and it could also reduce the power requirements for processing these materials. Generally for metal powders, with a radiation source of shorter wavelength, a higher percentage of incident radiation is absorbed as compared to a radiation source with longer wavelength (Ready, 2001). There are also other factors like particle size, compaction of powder and type of material which affects the absorption of radiation by a metal in the powder 
form and therefore for new materials, processing window is obtained experimentally to reduce different problems like balling and porosity etc. (Kruth et al, 2005).

The absorption of radiation by a metal powder depends on different factors. Ang et al (1997) showed that a rough surface increased laser absorption compared to a flat surface. It was shown that the cumulative absorption coefficient of two different wave lengths $(248 \mathrm{~nm}$ and $690 \mathrm{~nm})$ of laser radiation on a solid aluminium target at room temperature $\left(20^{\circ} \mathrm{C}\right)$ was also dependant on the wavelength of incident radiation. More energy was absorbed with a shorter wave length of $248 \mathrm{~nm}$ compared to a longer wavelength of $690 \mathrm{~nm}$.

An experimental investigation into the laser radiation absorption as a function of temperature has been carried out by Bonch-Bruevich et al (1968). It was shown that laser radiation absorption increased with increasing temperature and surface roughness.

Tolochko et al (1997) studied the effect of laser radiation on the surface of the powders, in particular, the change in the absorption coefficient $\left(\mathrm{K}_{\mathrm{abs}}\right)$ during laser treatment. A Nd:YAG laser $(\lambda=1060 \mathrm{~nm})$ was used to irradiate nickel alloy PG $12 \mathrm{~N} 02$. The laser was operated with a pulse duration of $5 \mathrm{msec}$ and the pulse repetition frequency ranged up to 10 $\mathrm{Hz}$. The diameter of the focal spot on the surface of the powder was about $2.5 \mathrm{~mm}$, which ensured a peak power density up to $10^{4} \mathrm{~W} / \mathrm{cm}^{2}$ in a pulse and an average power density up to $4 \times 10^{2} \mathrm{~W} / \mathrm{cm}^{2}$. Nickel alloy was exposed to radiations of sufficient power that the powder particles sintered together. The fine particles and coarse particles successively underwent complete fusion with an increase in the heating temperature and the value of $\mathrm{K}_{\mathrm{abs}}$ changed during this process.

The value of $\mathrm{K}_{\mathrm{abs}}$ was observed to increase during the initial stage of heating of the powder (Figure 2-9a and 2-9b). This was because the increase in temperature results in increased radiation absorption and thus higher absorption coefficient (Ready, 2001). Table 2-1 shows absorption coefficients for different powders, from which it is evident that radiation absorption is sufficiently increased in the powder form. 


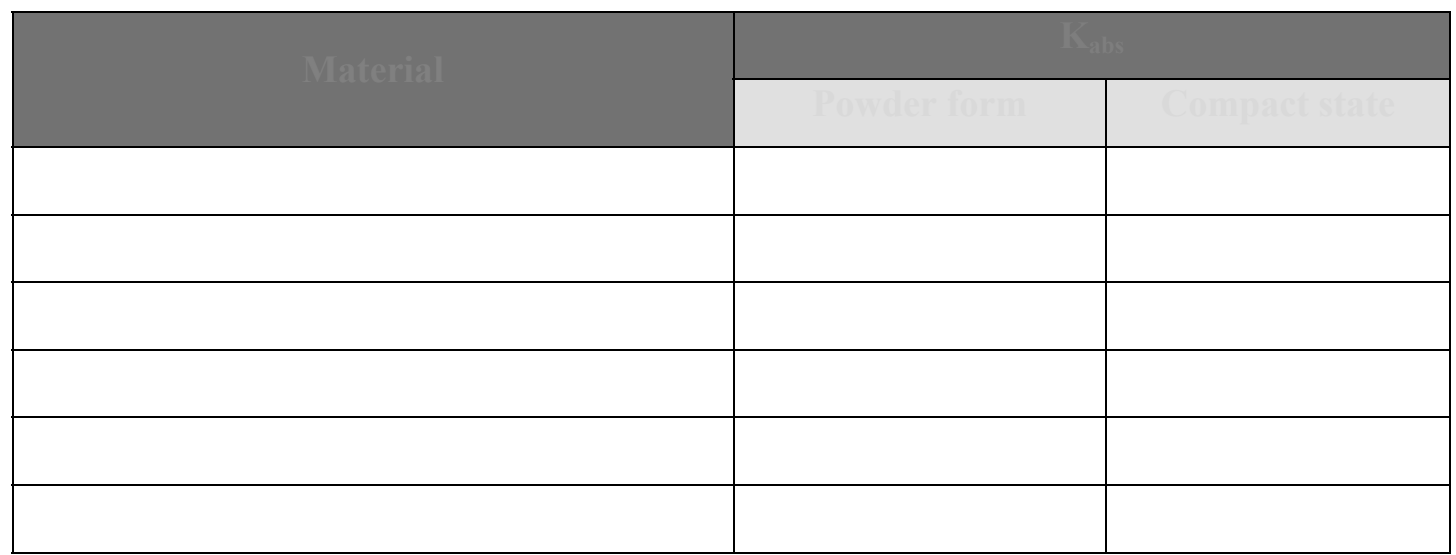

Table 2-1: Absorption coefficients (Kabs) of different materials in powder and bulk form (Tolochko et al, 1997)

Further increasing the laser power results in sintering of the powder and the $K_{a b s}$ continues to increase. Bridges are formed between the particles which retain their original shape and size. From this stage onward there are two different possibilities:

In one case a thermal equilibrium is established if the power density is relatively low. The amount of heat absorbed by the sintered powder equals the amount of heat given off to the environment. In this case the state of the particles is stabilized and the absorption coefficient assumes an almost constant value (Figure 2-9 (a)).

In the other case if the laser power is very high, the particles begin to fuse intensively forming a melt pool of half melted metal. The formation of half melted compact metal in the powder causes $\mathrm{K}_{\mathrm{abs}}$ to begin to decrease (curve IV in Figure 2-9b). The absorption coefficient ultimately stabilizes when the particles are completely melted as shown in Figure 2-9(b). 
Figure 2-9: (a-c) Change in absorption coefficient of Nickel alloy (PG12N02) during laser treatment with a radiation power density of 100 (a and c) and 250W/cm2 (b) (Tolochko et al,

The value of $\mathrm{K}_{\mathrm{abs}}$ was also observed to decrease at the beginning of heating (curve VI in Figure 2-9c). If the particles subjected to laser treatment consist of very small and very large particles i.e. large difference between the sizes of particles then in this case the smallest particles will quickly melt, while the large particles will remain unaltered. This creates some molten metal and some large particles. After this, $K_{a b s}$ will change approximately in the manner described above. There are certain distinctive features to the behaviour of particles during sintering and re-melting.

\subsubsection{Heat Transfer in the SLM Process Powder Bed}

The thermal conductivity of a material is an indication of the material's ability to transfer heat. Thermal conductivity depends upon the temperature of the material and its state (solid, liquid and gas). According to Fourier's heat transfer theory, the heat in a material flows via conduction due to the thermal gradients. Apart from the state of the material, type of material i.e. bulk solid or powder form changes the way heat transfer occurs in the material 
(Karapatis, 2002). A powder bed used in the SLM process is non-homogeneous in nature and conduction heat transfer only occurs through the contact points between the adjacent particles as shown in Figure 2-10.

Figure 2-10: (a) Transfer of heat between powder particles via conduction \& (b) heat distribution within powder bed (Mumtaz, 2008)

A highly compacted powder would have greater number of contact particles thus increasing conductivity through the power bed. In Figure 2-10a, $\mathrm{I}_{0}$ indicates the incident radiation and $\mathrm{Q}_{\mathrm{c}}$ shows the heat transferred between the particles. A smaller portion of the heat could be transferred via radiation in the gaps between the particles. Figure 2-10a does not mention the radiation absorption from multiple reflections when the incident radiation is trapped in the gaps between the particles (Fisher et al, 2003). This is already explained in detail in section 2.3.3.1. Figure 2-10b shows the heat transfer and decreasing temperature zones away from the point of laser material interaction. Heat transfer from the heated powder bed or melt pool to the air is relatively low as compared to the heat transfer through the powder bed because of air being a poor conductor.

The amount of energy required to heat and melt a metal powder particle with radius $r_{p}$, volume $v_{\mathrm{p}}$ and density $\rho$ is defined by equation (2.1).

----- (2.1) (Karapatis, 2002)

Where $\Delta \mathrm{H}$ is the amount of energy required to melt, $\mathrm{C}_{\mathrm{p}}$ is the specific heat capacity, $\Delta \mathrm{T}_{\mathrm{m}}$ is the temperature difference between the ambient and material melting temperature, $\mathrm{L}_{\mathrm{f}}$ is 
the latent heat of fusion and $\mathrm{E}_{\mathrm{m}}$ is the melt energy. According to this equation, the smaller the difference between the material melting and ambient temperature $(\Delta T)$, then lesser will be the energy required to melt the material. Elsen (2007) calculated the amount of energy required to melt stainless steel powder with powder bed pre-heating of $500^{\circ} \mathrm{C}$ was $22 \%$ less than without any pre-heating (chamber temperature). In some cases a powder bed temperature of 200$250^{\circ} \mathrm{C}$ is noted to have 'caking' or solid state sintering effect (Das, 1998) (Elsen, 2007). The reason behind this is that smaller particles tend to melt quickly as compared to larger particles thus sintering together through the contact points to form caking.

\section{Energy Losses}

The energy supplied by laser radiation is absorbed, reflected or transmitted. Some portion of this energy is utilised in raising the temperatures of this powder and changes its phase while some is lost through different means. Figure 2-11 shows the energy losses during the laser processing.

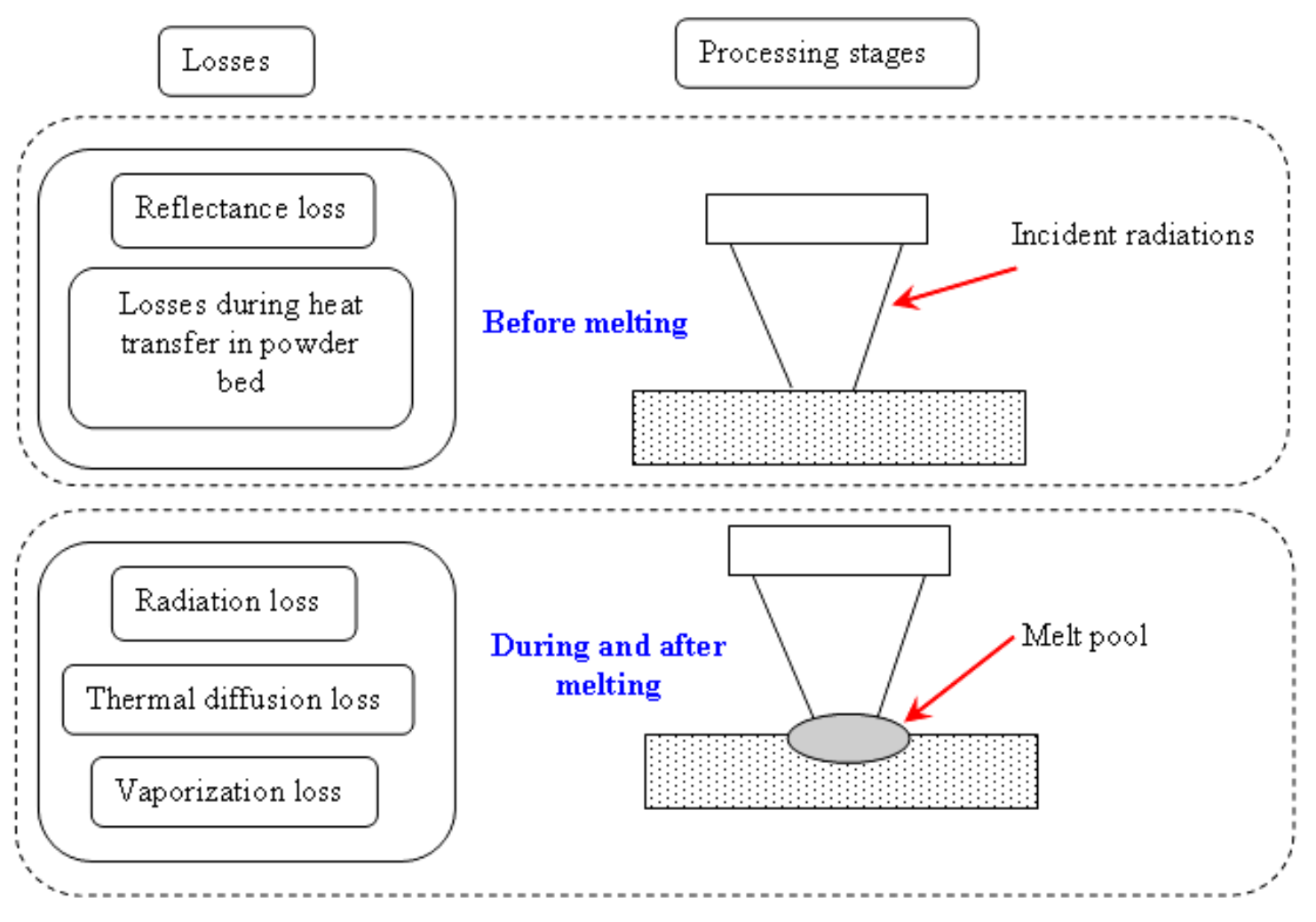

Figure 2-11: Energy loss during laser material processing in powder 


\subsubsection{Melt Pool Dynamics}

The changes in the behaviour of metal in the molten form with the substrate, input energy, temperature gradients and the resulting surface tension variation is the study of melt pool dynamics in laser material processing. All these factors affect the formation of a single bead and single layer which acts as building block in the SLM process. The shape, size (width and height) and structure of the beds is affected by the wettability of the material, capillary instabilities and thermocapillary flows during the molten stage of the metal. Studying the effect of these factors is crucial to the quality of the parts and these are explained in detail below:

\section{Wettability}

Wettability is the spreading behaviour of a liquid on a solid. Wettability can adversely effect the inter-layer connection, porosity and final strength of the parts (Singheiser et al, 2001) (Steen, 2003). The contact angle of the liquid drop on the solid surface determines the wetting behaviour of the liquid on that substrate. The same is true for metals in liquid form on the substrate during the laser processing. Equation (2.2) is the Young's equation which defines

the contact angle of liquid on solid with the surface tension between liquid and solid in air (Figure 2-12).

Figure 2-12: Three phase equilibrium of wetting liquid on a solid (adapted from Eustathopoulos et al, 1999)

(2.2) (Eustathopoulos et al, 1999)

Where $\gamma_{S V}$ is the surface tension of solid in air, $\gamma_{L S}$ is the interfacial surface tension of liquid and solid and $\gamma_{L V}$ is the surface tension of liquid in air. According to this equation, the liquid will wet the underlying solid if $\gamma_{L V} \leq \gamma_{S V}-\gamma_{L S}$. Improved wetting occurs at contact angles 
smaller than $90^{\circ} \mathrm{C}$ (Agarwala et al, 1995) (Extrand, 2003). High solubility of the component materials (in alloys) in the liquid phase improves the wetting behaviour of the alloy and also assists in the re-arrangement of the material compositions within the melt pool (Kruth, 1991).

In the SLM process, different factors affect the wetting behaviour like the temperature gradients, time it takes the molten metal to re-solidify, oxidation of the substrate and previous sintered layer and recoil pressure. As the temperature increases, $\gamma_{L V}$ reduces which according to equation (2) reduces the contact angle and improves wettability (Kruth et al, 2003). In the SLM process, the melt pool solidifies very quickly and reduces the time the material is kept in molten state. Increasing this time also increases the wettability of the material.

Because liquid metals have poor wetting properties on solids with an oxide film, the addition of de-oxidizing agents is also known to increase the wetting property (Das, 1998).

\section{Capillary Instability}

Capillary instability relates to the instability of a liquid on a solid where the liquid splits into smaller entities in order to reduce the surface tension variations. When the molten metal in the SLM process shows this behaviour, it is called 'balling'. As its name indicates, the liquid metal splits into droplets instead of forming a uniform bead or line to minimize the surface tension (Morgan et al, 2004). As surface tension is a function of temperature, due to the non-uniform heating during the laser processing temperature gradients create surface tension variations across the melt pool. The natural tendency of the liquid metal is to reduce this surface tension variation which results in break up of melt pool into smaller droplets. The non-wetting behaviour of molten material with the underlying surface also increases the chances of balling (O'Neill et al, 1998). Mumtaz (2008) observed smaller spheres to the side of the bead. It is not clear that the smaller spheres attached to the side of bead is balling or satellite formation (Mumtaz, 2008), it needs further investigation. Figure 2-13 shows balling phenomena where smaller balls attached to the side of the bead and actual melt pool splitting into larger droplets. The splitting of melt pool into balling or spheres is discussed in detail in Chapter 8. 


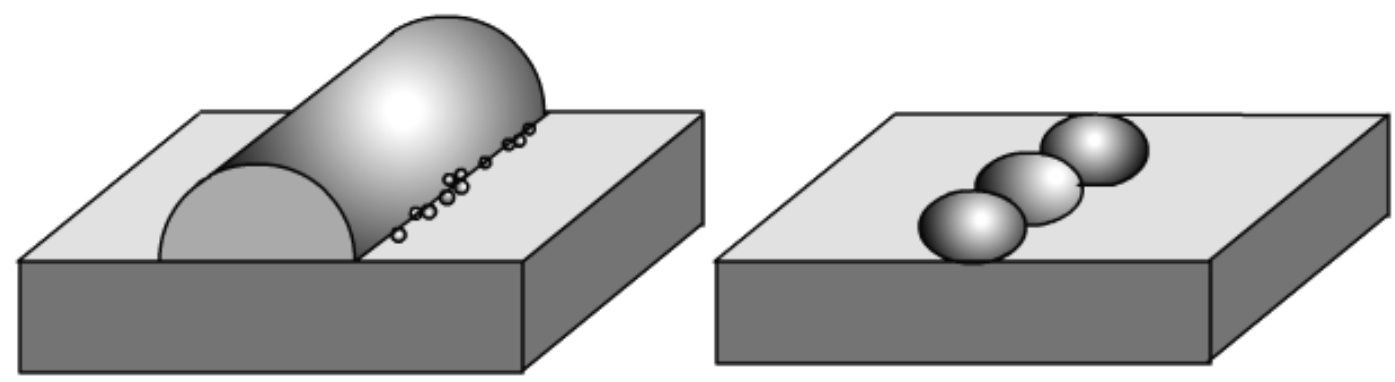

Figure 2-13: (a) Small spheres attached to the side of melt pool (b) Melt pool splitting into larger droplets

Kruth et al, 2004 examined the cause of this phenomenon in the SLM process and found that when the total surface of the melt pool becomes larger than that of a sphere of the same volume then balling occurs. Figure 2-14 shows the schematic when the balling phenomena can occur in the melt pool during the SLM process. This shows that in order to avoid balling, the length to diameter ratio of the melt pool should be kept to a minimum (below 2.1). This is possible if the molten material quickly re-solidifies behind the laser, keeping the length of melt pool to a minimum. The molten material in the melt pool processed using a pulse laser quickly solidifies and thus has a lesser chance of balling. A pulse laser is also known to have less residual effect due to a smaller heat affected zone (Mumtaz, 2008). If the time taken for a molten material to solidify is larger than the time it takes to break up into balling, then balling will occur. Molten material with higher viscosity is known to increase the break up time but if some balling occurs, the molten spheres are very large and far apart (Rombouts et al, 2006) (Donnelly and Glaberson, 1965) .

Figure 2-14: Transition form half cylinder to sphere (adapted from (Kruth et al, 2004)) 
Balling is one of the major problems in SLM processing. Balling can cause weak interlayer adhesion (Fuh et al, 1995), porosity in the parts (Hauser et al, 2005), non-uniform layers and hindrance in successive layer deposition (Kruth et al, 2003) and increase in surface roughness of the parts.

\section{Thermocapillary flows}

Apart from the wettability and balling issues, there is another phenomenon which also occurs during laser melting of material, which is thermocapillary flows in the molten material. These thermocapillary flows are called Marangoni flows. Marangoni flow is from a region of higher surface tension to a lower surface tension. These flows are caused by the non-uniform temperature variation within the melt pool during laser processing, which induces a surface tension gradient and induces a convective fluid flow within the melt pool. Two different scenarios can exist within a melt pool exposed to such surface tension gradients i.e. the flow of

fluid can be outwards from the centre of the laser spot or inwards towards the centre of the laser spot. According to Mills et al (1998), the sign of the surface tension gradient $\left(\mathrm{d} \gamma_{\mathrm{LV}} / \mathrm{dT}\right)$ determines the direction of fluid flow within the melt pool and hence defines the topology of the re-solidified bead (a line of re-solidified material produced during a single pass of laser on the material). Figure 2-15 shows the temperature and surface tension gradient and the resulting Marangoni flow within the melt pool. A wider and shallow melt pool results if the surface tension gradient is negative i.e. the fluid flows outwards from the centre of the laser spot as shown in Figure 2-15(a). Similarly a narrow and deeper melt pool results if the surface tension gradient is positive i.e. the fluid flows towards the centre of laser spot.

Figure 2-15: Schematics presentation of Marangoni convection in a melt due to the presence of a surface tension gradient ( $\mathrm{d} \gamma \mathrm{LV} / \mathrm{dT})$ (Mills et al, 1998) 
The surface tension gradient $\left(\mathrm{d} \gamma_{\mathrm{LV}} / \mathrm{dT}\right)$ of pure metals and many alloys is negative (Rombouts et al, 2006), which creates a narrow and shallow melt pool and was also observed in the processing of gold $(\mathrm{Au})$ powder in this current research work which is discussed in detail in Chapter 8. Apart from the Marangoni flow, buoyancy can also affect the fluid flow within the melt pool due to density variations in different parts of the melt pool. Gravity is the driving force behind buoyancy but due to the small size of the melt pool in the SLM process (less than $1 \mathrm{~mm}$ in width), it is considered to have minimum effect (Kruth et al, 2007). With a larger melt pool in welding, the effect of buoyancy is more pronounced (Mills et al, 1998). As most of the lasers follow a Gaussian energy distribution, the energy at the centre of the laser spot is much higher as compared to the edge of the laser spot. Due to this very high thermal gradient, strong Marangoni flows occur and tend to dominate the flows within the melt pool. The strength of the Marangoni flow can be estimated by equation (2.3):

Where, $\mathrm{dT} / \mathrm{ds}$ is the thermal gradient at position $\mathrm{s}, \mathrm{L}$ is the length of the melt pool, $\eta$ is the viscosity and $\delta$ is the thermal diffusivity. A higher Marangoni number $M_{a}$ indicates a greater Marangoni flow influence in the molten material. Figure 2-16 shows the Marangoni flow in the melt pool during laser processing.

Figure 2-16: Marangoni convection within the cross-section of the melt pool (Mumtaz, 2008)

The flow of material within the melt pool influences its shape and stability i.e. whether a narrow and deep or wider and shallow melt pool is produced (Pinkerton et al, 2006). 


\subsubsection{Melt pool Ejection}

During the SLM process, material could be heated above its vaporization temperature. This high temperature can transform material into its vapour phase. This vapour can get ionized when this vapour interacts with the incident laser. The ionization generates a plasma plume which is present over the surface of the powder bed. The plasma plume is known to reduce the effect of incident radiations on the powder bed (Duley, 1999). Over time the plasma plume absorbs more and more of the incident radiations and creates a heat shock wave which travels in all directions from a certain height above the powder bed. There is a high pressure region behind the plasma plume which generates pressure on the molten material also known as 'recoil pressure' (Morgan et al, 2004). This high pressure forces the melt pool to forcefully spread over the surface known as 'enforced wetting' (O’Neill et al, 1998). This high recoil pressure can also cause the molten material in the melt pool to eject which is called 'spatter'. An increase plasma plume which is an effect of increased heat input is accompanied by increase in the melt pool ejection (Park and Rhee, 1999). Semak and Matsunawa (1997) concluded that low bulk density material powders are more prone to spatter as compared to the high density materials. It was also noted that the viscosity of the molten material influences melt ejection form the melt pool. The spatter or ejection of material from the melt pool reduces the amount of material in the melt pool (Kruth et al, 2004) which could create discontinuities and could create porosity in the final part. Spatter can also hinder the material deposition process and could affect layer quality. Rombouts (2006) presented another explanation for spatter formation. According to this explanation, due to the high temperatures in the laser melting, the gases entrapped in the powder bed could be superheated which would eventually explode and eject the molten material from the melt pool. Figure 2-17 shows the molten metal ejecting from the melt pool during laser melting operation. 
Figure 2-17: Melt pool ejection as a result of high recoil pressure on melt pool surface (Mumtaz, 2008)

\subsubsection{Melt pool Shape}

It has been noted that the melt pool shape tends to flatten with a pulse laser which generates recoil pressure on the melt pool. In the studies conducted by Ohmura et al (1997), Morgan et al (2004) and Fischer et al (2005), it was noted that high peak powers can have a significant effect on the shape of the melt pool. Kruth et al (2004) found that high peak powers suppress balling by forcing the molten material to flatten over the substrate, improve its wetting angle. Mumtaz et al (2008) also observed the decrease in contact angle during Nd:YAG pulse laser melting. Figure 2-18 shows melt pool shape modification due to recoil pressure. Although it has been observed that high peak power improves wetting and flattens the melt pool. There has been no study on the amount of pressure generated by peak powers to utilise this in a controlled manner to improve wetting and reduce the balling effect. 


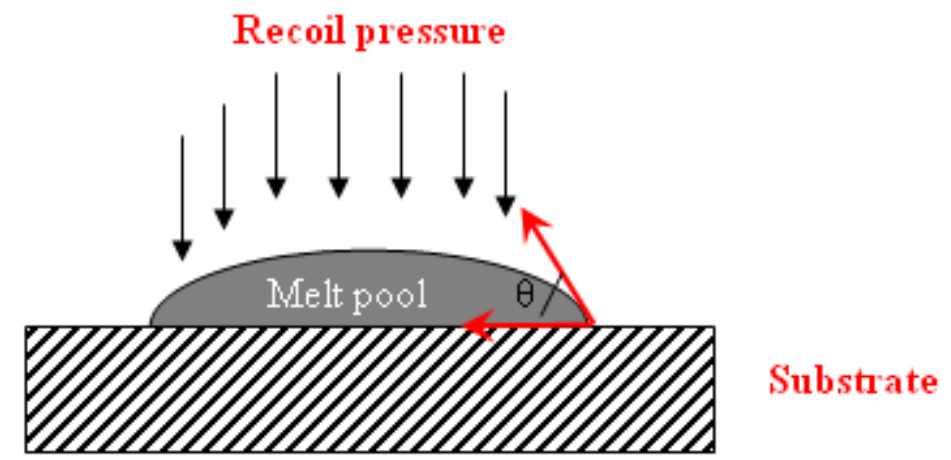

Figure 2-18: Melt pool shape modification due to recoil pressure

\subsubsection{Environmental Effects}

The environment in which metal powder is being melted and allowed to re-solidify is very important to the property of the parts produced. Different environmental factors which affect part property are powder bed pre-heating, oxidation and inert gases, which are discussed in detail below.

\section{Oxidation}

Many laser based processes such as welding, surface treatment and SLM are associated with an un-wanted phenomenon called oxidation. The common solution employed for this problem is to use (a) shielding gas and is one of the important parameters in welding (Grevey et al, 2005). With laser processing as in SLM, the laser energy absorption coefficient of the oxides is higher than that of the metal, which increases the temperature and overheats the material. According to Rombouts (2006), the higher the temperature and overheating of the metal, the higher will be the oxidation in molten metals. This is shown by equation (2.4) (Xie and Kar, 1999) (Yakovlev et al, 2005).

(2.4) (Rombouts, 2006)

$\Delta \mathrm{G}$ is defined as the free energy of the reaction and its increasing values indicate higher oxidation in the molten metal, $\mathrm{R}$ is the universal gas constant, $\mathrm{T}$ is the absolute temperature and $\mathrm{P}_{\mathrm{O} 2}$ is the partial pressure of gas. As discussed earlier in section 2.2.3.3, wettability is affected by the surface oxidation in the laser processing and SLM process, which 
creates different problems like porosity and reduced mechanical properties (Morgan et al, 2004) (Gower et al, 2005).

\section{Inert environment and gases}

An inert environment or a shielding gas is often the solution to the oxidation problem in laser processing of metal. Different methods are used for shielding purposes. One is the nozzle feed method in which inert gas is fed from a laser nozzle directly onto the point where metal is made molten by the laser. The other method is to evacuate the whole chamber and fill it with an inert gas. Although the nozzle method is employed the chamber method is more effective in protecting the melt pool from oxidation. The drawback in filling the whole processing chamber is that it is more costly and requires more setup time as compared to the nozzle feed method.

There are a variety of inert gases that are used for shielding molten metal including helium (He), argon (Ar) and nitrogen $\left(\mathrm{N}_{2}\right)$. Helium is lighter than $\mathrm{O}_{2}$ therefore making it difficult to protect the molten metal. With helium, if a very small percentage of oxygen is present, it will be on the surface of the molten metal and thus oxidize it. Nitrogen gas on the other hand reacts with some of the metals to make nitrides such as $\mathrm{Ti}, \mathrm{Cr}$ and $\mathrm{Mg}$ which are present in some alloys like Inconel 625. The reaction of nitrogen with these metals affects the

mechanical properties of the final part (Kou, 2003). Argon gas is heavier than $\mathrm{O}_{2}$, making it the best option for shielding purpose (Messler, 1999). Many of the commercially available metal processing systems such as MTT Realizer, EOS M270 etc uses argon gas for shielding.

\subsubsection{Thermal Effects in Metal Parts}

SLM is a very fast heating and cooling process compared to the conventional casting processes. Whether a pulse or continuous laser is used, the metal is kept in the molten state for a fraction of a second. This short time and high thermal cycle rate induces stresses in the part and cause dimensional inaccuracies, solidification cracking and layer de-lamination etc (Karapatis, 2002). Due to high thermal gradients, there are always some residual stresses in the parts. These parts can be post processed and these stresses can be relieved in the furnace (Santos et al, 2006). 


\section{Shrinkage}

Shrinkage in the material occurs when it is allowed to cool and solidify from the molten state. Different metals undergo different amount of shrinkage and therefore SLM has a different effect on the processing of these metals (Jeng and Lin, 2003). The shrinkage is known to create microstructural and bonding problems as the previous layer in the SLM part is already solidified and relatively much cooler (Karapatis et al, 1998). Apart from changing the processing parameters such as reducing laser spot size, reducing pulse duration and increasing scan speed (Pohl et al, 2001), the thermal shrinkage can also be reduced by powder bed preheating (Sexton et al, 2002). Some companies are striving to develop a minimum shrinkage or near zero shrinkage powder such as EOS Direct Steel 50 (Karapatis et al, 1998). Most of the commercially available system(s) incorporate the pre-determined material shrinkage values thus keeping the final product in the dimensional limits.

\section{Solidification Cracking}

Solidification cracking or hot cracking results from the inability of the solidifying metal to accommodate for the thermal shrinkage. Solidification cracking can also be because of the thermal residual stresses due to high temperature cycles (quick heating to higher than melting temperatures and un-controlled cooling) in the SLM process (Kruth et al, 2004) (Shannon and Li, 2004) (Zhong et al, 2005). Similar to shrinkage, solidification cracking can be reduced with the powder bed pre-heating (Sexton et al, 2002).

Because of very low powder bed pre-heating (about 200 to $300^{\circ} \mathrm{C}$ ) in most commercial systems, shrinkage and solidification cracking is still one of the problems especially with larger parts.

\subsubsection{Modelling of Melt Pool}

The physical processes that take place during laser melting include heat transfer into the material, radiative, convective and conductive heat transfer and thermodynamics of phase changes (Leung 2001; Yang et al. 2001).

Many simple analytical models have been presented which determine the temperature profiles or geometry of laser treated tracks during laser melting. These models explain and predict the effect of laser induced melting of material (Cheng \& Kar 1997; Lawrence et al. 
2001). These models simulating a continuous Gaussian mode for the laser beam are mainly based on the classic analytical solution derived by Carslaw \& Jaeger (1959) in terms of integrals over the surface of the expression for a point source. These models focused on laser melting of metallic materials. In addition, in order to achieve satisfactory results, carefully selected laser process parameters approaches were usually employed. Li et al. (2004a) developed an analytical model with both a volumetric heating source and a surface heating source to predict the melt cross-sections of an $\mathrm{Al}_{2} \mathrm{O}_{3}$-based refractory during $\mathrm{CO}_{2}$ laser treatment; however, no heat radiation, heat convection and phase change were considered. Li et al. (2004b) expanded the two-dimensional numerical model to develop a three-dimensional numerical model for a convection-diffusion phase change process during laser melting of refractory ceramic materials. The exact theoretical model was applied to analyse the thermal field using numerical approaches. More recently, Li et al. (2004c) developed a threedimensional mechanical FE model to investigate thermal stresses and their implication on cracking during laser surface melting of ceramic materials and analysed the effects of latent heat of fusion and fluid flow of the melt pool on the thermal stresses.

Roberts et al (2009) presented a three-dimensional FE model of temperature fields during laser melting of an isotropic material. A moving heat source model was created expanding from single to multiple layers. It was observed that the heated regions undergo rapid thermal cycle which results in the thermal stresses in the parts. The addition of more layers and subsequent laser scanning alter the temperature distribution and stress build-up in that layers. The stresses induced in the single metallic layer during SLM are presented by Matsumoto et al (2002). The single layer was observed to warp during the laser passing and the simulation showed a pattern of compressive and tensile stresses in the layer during SLM. The deflection in the layer was dependent on the length of the scan tracks; where longer scan tracks showed higher deflection. Apart from that, tensile stresses were observed at the edge of the previously solidified tracks which could be responsible for the resulting cracks in the parts. It was suggested that the scans should be reduced for larger layers which could reduce the thermal stresses in the parts and hence reduce damage to the layers.

Gusarov et al (2007) studied the influence of processing parameters on the local temperature distribution in the laser powder interaction zone. A simplistic model of single scan on the powder layer was modelled. The processing parameters were taken for stainless 
steel 316L. The effect of temperature on the absorption properties could not be modelled due to the lack of available absorption data at melting or higher temperatures. A constant thermal conductivity $(20 \mathrm{~W} / \mathrm{mK})$ was assumed as no reliable experimental data was available for the variation in thermal conductivity during and after phase change in the powder bed. The flows within the melt pool were also not taken into considerations which gave a different shape of the melt pool in modelling as compared to that experimentally observed. These flows within the melt pool are driven by the thermal and surface tension gradients as discussed in details in section 2.3.3.3. From the model developed in this research work, it was concluded that balling at high velocities (above $200 \mathrm{~mm} / \mathrm{s}$ ) can be because of the Plateau-Rayleigh instability of a liquid cylinder. It was concluded that melt pool could be stabilized by either reducing the length-to-circumference ratio or increase in the contact between the melt pool and substrate.

The Finite Element models presented above have been used to predict some of the behaviours of processed material under different processing conditions. However, the nonlinearities associated with the powder material (such as change in the radiation absorption with particle size and compaction) and temperature dependant properties (such as radiation absorption, surface tension gradients, thermal conductivity and thermocapillary flows) have forced the researchers to model the thermodynamics of melt pool with various assumptions. Apart from that, the change of phase of powder material into molten material during the laser processing also changes the radiation absorption and thermal conductivity non-linearly during laser processing.

\subsection{Summary}

The selection of DMLF process depends on many factors including the power requirements, laser parameters, type of inert environment and last but very importantly the type of material being processed. The wire feed, powder feed and powder bed process selection depends on the type of job requirement. Wire feed and powder feed can be used for repair operation of the components, however the wire feed method is not often used for part production. The powder bed deposition processes are not feasible to be used for part repairs but used for new parts production. The powder bed deposition process has high powder utilization, whereas the powder fed process has complications like consistent powder flow rate which directly influences the part quality. 


\section{Chapter 3}

\section{DIGITAL MANUFACTURING IN MEDICINE AND DENTISTRY}

\subsection{Digital Manufacturing in Medicine}

Digital manufacturing has changed the way manufacturing is done these days. Starting from scanning an existing physical model or modelling to manufacturing through Computer Aided Manufacturing (CAM) using additive and subtractive technologies, every step can be digitized. Each phase of this digital process involves multiple checks before final qualification and manufacturing. This section explains the digital manufacturing process in detail.

\subsubsection{D Medical Scanning}

The medical/ dental sectors have always been looking for better ways of understanding the condition of individual patients before any decision on their treatment could be made. Computer Tomography (CT), Ultrasound Imaging and Magnetic Resonance Imaging (MRI) have been most commonly used to assist doctors and surgeons for a true 3D representation of the human body. Advanced imaging such as Positron Emission Tomography (PET) has enabled capture of the physiology as well as shape data.

Apart from these technologies, different white and red light laser scanners have also been used for capturing 3D surfaces. The desire for precise diagnosis and non-invasive treatments has always boosted the development of new techniques for better results. Apart from the hardware side, different software for example ScanIP from Simpleware (Simpleware, 2009), AnatomicsRx from Anatomics (Anatomics, 2009) and MIMICS from Materialise (Materialise, 2009) have also been developed to transform the data from these technologies to virtual 3-D models. These softwares stack the hundreds of slices from CT and MRI into a single volume. Some of the software modules like "SurgiGuide drill guides" and "SimPlant" from MIMICS for medical and dental solutions provide the doctors and surgeons with the virtual 3D planning environments in which they can model the prostheses and dental implants etc. The 3D models developed in the virtual environment from medical imaging techniques 
can also be physically fabricated by different techniques. Figure 3-1 shows reconstruction of a patient's skull using data from the CT scans in the MIMICS software.

Figure 3-1: $\quad 3 D$ reconstruction and implant design in MIMICS (Gibson, 2005)

This section explains these technologies in detail as well as their applications in different medical cases etc.

\subsubsection{Computerised Tomography (CT)}

Computer Tomography (CT) is also known as Computed Axial Tomography (CAT). CT scanning was developed in the mid 70's and is the most patient friendly and one of the most widely used imaging devices. CT scanning is based on the same principle of X-ray where X-rays penetrate through the body before being captured by a detector. This allows the CT scans to differentiate between the bone and soft tissue with good contrast. Rather than obtaining a single image on the film as in conventional X-ray, the CT scan measures a complete density profile of the targeted area. CT scans are especially useful in accurately finding the location, size and spread (including secondary growth) of cancerous mass or tumours. Other pathological changes in the body tissue or organs can also be precisely defined to greatly improve the clinical outcome. 


\subsubsection{Ultrasound Imaging}

Ultrasound imaging also known as Ultrasound scanning or sonography is a fast and radiation free imaging technique. Ultrasound is excellent for non-invasively imaging and diagnosis of a number of conditions, without exposing the patient to harmful X-ray radiations (Gibson, 2005).

\subsubsection{Magnetic Resonance Imaging (MRI)}

Magnetic Resonance Imaging (MRI) is based on the principle that different materials resonate at different magnetic field strengths. MRI uses magnetic energy and radio waves to create cross-sectioned images or slices of the human body. MRI scans can provide images with accurate contrast of soft tissues like gray and white matter in the brain and small metastatic lesions in the liver. MRI can also be used to examine injuries of joints, blood vessels and different bodily organs (Gibson, 2005).

\subsubsection{3D Scanners}

3D laser scanning is a surface capturing/ digitizing technology that captures the physical shape of an object in the form of multiple points. The main advantage with laser scanning is its non-contact method, enabling it to scan and measure very fragile parts. Initially contact methods like touch probe Coordinate Measuring Machines (CMM's) have been used because of their high accuracy but it takes a lot of time to scan large irregular surfaces. With the current improvements in the accuracy of the laser based non contact methods, ease of use and less time consumption, their use has now considerably increased.

\subsection{Rapid Prototyping (RP) and Rapid Manufacturing (RM) in Medicine}

The manufacturing flexibility provided by RM is increasingly finding its use in a variety of different fields. Figure 2-1 shows that the medical/ dental sector is the third leading sector which has embraced RM technologies. Different manufacturing methods have been used in medicine and dentistry but the introduction of Rapid Prototyping (RP) and Rapid Manufacturing (RM) technologies has re-defined the way doctors and surgeons perceived manufacturing. Rapid Manufacturing technologies have been increasingly used for different medical applications to create accurate physical models directly from medical imaging data (Gibson, 2005) (Chelule et al, 2000) (Mcgurk et al, 1997) (Swaelens and Kruth, 1993). These 
physical models can help surgeons to practice complex surgeries in order to avoid any potential complications. For example Figure 3-2 shows (a) an Stereolithography (SL) model of a patient's skull to plan for precise Osteotomy and (b) mandible (lower jaw) reconstruction.

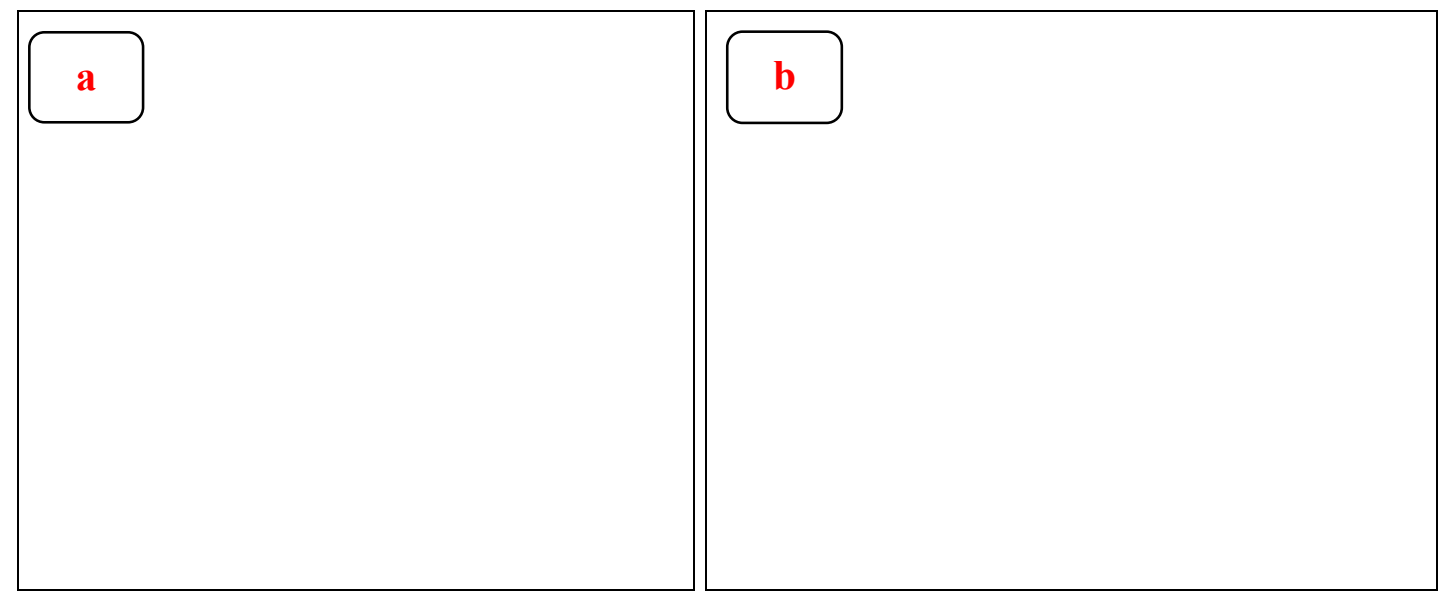

Figure 3-2: (a) Osteotomy (bone cutting) planning and (b) mandible reconstruction on a RP model (Gibson, 2005)

Figure 3-3 shows an SL model of a tumour in a human skull made from two colours to differentiate the tumour (in red) from the skull. CT scan contrast was used to differentiate tumour from normal tissue and the 3D model was segmented and checked in the virtual environment. The differentiating colour was achieved by a special resin which changes colour with multiple exposures to Ultraviolet (UV) light.

Figure 3-3: Model of a patient's skull made by two-colour stereolithography indicating the shape and position of a tumour (Vandenbroucke, 2008) 
It can be seen from Figure 3-2 and Figure 3-3 that physical modelling of the 3D imaging data either from CT or MRI etc. can be extremely beneficial in diagnosing and treating medical and surgical cases more accurately. This is similar to manufacturing where prototypes (sample parts) are prepared for testing, look and physical feel of the virtual product. Also, pre-planning complex procedures can considerably reduce the risks involved and improve the surgical outcomes of these operations.

Rapid Prototyping (RP) models can also be used as master patterns for moulds used to create prostheses. Due to the flexibility of RP, complex pattern shapes can be produced very easily ensuring a high degree of precision and customization. This reduces the actual production time of the final parts which is one of the important factors in surgeries.

Apart from making physical prototypes for virtual models, doctors and surgeons can use RP and RM for direct use parts and tools. Some examples include custom built hearing aids (Figure 3-4) and personalized guides for drilling in complex spinal surgery (Figure 3-5). The hearing aid provides a custom fit which makes it easier to hold the hearing device in the ear. The custom drill guide can also be used as to place oral implants and jigs can help align bone segments in their place. These surgical aids are designed according to patient-specific anatomical features, which is typical by a negative of the place where is it placed.

a

Figure 3-4: Hearing aids by Materialise (a) hearing aid design (b) RP model (Materialise, 2009) b 
Figure 3-5: (a) Virtual planning of a complex spinal surgery using a personalized drill guide for spinal screw insertion (b) SLA made drill guide being used during spinal screw placement in a lumbar vertebra (Brussel, 2002)

RM of implants is becoming more and more common. The processing of biocompatible metals like titanium for implant manufacturing by SFF processes have proved their feasibility to be used in the medical field. Figure 3-6 shows some examples of high quality metallic prostheses produced by metal. Figure 3-6a shows a cranioplasty plate for skull reconstruction made from titanium provided by MCP HEK. Figure 3-6b shows a hip replacement implant from titanium using Electron Beam Melting (EBM).

a

b

Figure 3-6: (a) Cranioplasty plate for skull reconstruction made by SLM of titanium (Source: (MCP HEK, 2009)); (b)Tri-flange implant for hip replacement surgery made by EBM of titanium (Source: (Arcam, 2009)) 


\subsection{Digital Manufacturing in Dentistry}

The conventional dental methods are time consuming, labour intensive and involve too many steps. First of all an impression of the patient's mouth is taken and a plaster model is made from the impression. After this a wax pattern of the desired restoration is created and manually sculpted to the required nearest shape upon the plaster model created earlier. This created wax pattern is then used in the investment casting process to create metallic restorations in a suitable metal or alloy. The casting process by itself is a lengthy process and involves post processing operations to the finished final part. Apart from the actual process of manufacturing and the time it takes, due to the complex structure of the dental implants, it is very difficult to create even the wax pattern to the actual shape of the impression. This results in many patients coming back for re-adjustments. In the conventional dental restoration or implant manufacturing process, a patient would give his/her impression and would have to wait for a few days before they could even try their restorations. Any changes at that stage also increased the time to treat a single patient and multiple appointments were a routine matter.

The digital methods in manufacturing have matured enough to be used in various fields. The dental field has very recently started taking advantage of the digitization process both in 3D shape and object capturing and the manufacturing process (Leu and Delli, 2008). Due to the improvement in the accuracy of these processes, the digital methods are replacing the traditional processes in dentistry.

\subsubsection{Dental Scanning}

Manufacturing dental restoration would take a few days with the conventional manufacturing technologies as explained earlier. With the new digital scanning techniques available commercially, the time span has been reduced to a couple of hours. Manufacturing of dental crowns and bridges is another area where the digital manufacturing methods have been introduced. The dental crowns and bridges need more than just the information about the damaged part of the teeth, rather the adjacent teeth are also important. These adjacent teeth are prepared for the crown or bridges to be installed. Traditionally the data about these teeth is obtained from the impression of the patient's mouth with the help of a plaster impression model. Presently there are two different methods for doing the same task. One method is to use the mechanical probe or no-optical method (Figure 3-7a), which is accurate but time consuming. The other method is the digital scanning with a non-contact scanner, which have 
become more accurate and many commercial scanners are available on the market. Figure 3$7 \mathrm{~b}$ and $3-7 \mathrm{c}$ shows a couple of non-contact type digital scanners. Laserdenta non-contact scanners have the limitation that it is not an intra-oral scanner and thus an impression model is developed first for scanning.
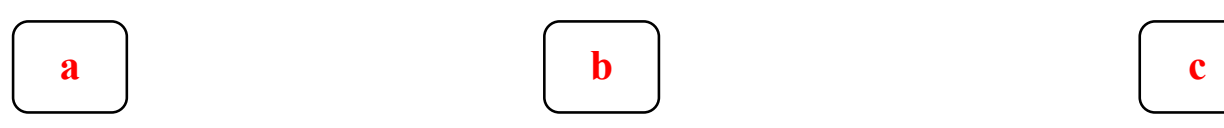

Figure 3-7: Digital digitizers/ scanners (a) InciseTM Scanner (Renishaw, 2009), (b) Laserdenta scanner (Laserdenta, 2009) and (c) CEREC® acquisition centre (CEREC, 2009)

However, Sirona has developed a non-contact scanning system (Bluecam) which in incorporated into the CEREC $^{\circledR}$ acquisition centre (Figure 3-7c). Bluecam scans part of the patient's complete jaw at a time and shows the 3D model instantaneously on a computer. A very good example of a complete digital scanning and manufacturing system has been given by CEREC $^{\circledR}$, which uses Sirona's Bluecam technology and table Computer Numeric Control (CNC) machine to manufacture dental restorations. Figure 3-8 shows a flow diagram of the process of digital dental manufacturing. First the patient's mouth is scanned by a hand held digital camera which directly transfers the 3D image of the teeth onto a 3D computer model. The software installed is used to create the desired restorations within a few minutes. The attached CAM system takes the data from the system and manufactures restoration for the patient. The whole process starting from patient's scan to implanting the restoration only takes a couple of hours which is considerably faster as compared to the traditional process which would involve multiple visits to the dentists. 


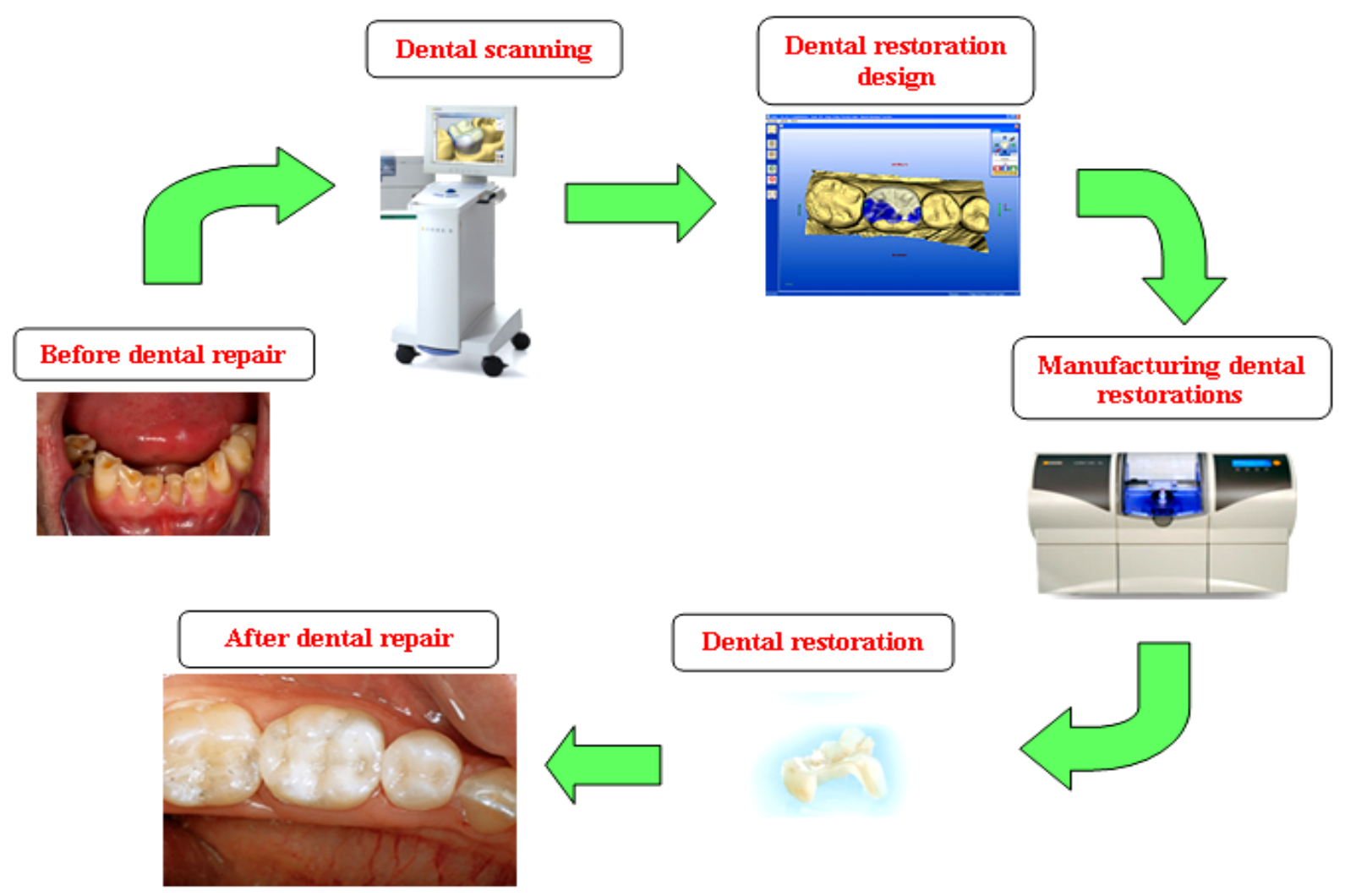

Figure 3-8: $\quad$ Process flow of digital dental restoration process

\subsubsection{Dental Materials}

Dental restorations have been manufactured by the lost wax method for decades. The lost wax method was very successful in that the complete prosthetics were manufactured from alloys. Acrylic veneers had been used to fuse onto the metal part for better aesthetics. In the 1960's a new manufacturing process was developed which used porcelain as the choice of material to coat metal parts (Cascone, 2003). These porcelain-fused-to-metal parts had good aesthetics as they were the closest looking to natural teeth and were very durable. The main advantage of the tooth made by this method is that it mimics the natural tooth which is brittle from outside and resilient from within, for this reason the porcelain-fused-to-metal restorations have become a widely used method up to this day. The ceramics currently used are zirconia, alumina and porcelain based. Ceramic like zirconia-based materials are very difficult to machine (Luthardt et al, 1999), so these material are used in their pre-sintered condition to mill and grind and shape them to the nearest net shape and size. After the machining process, these parts are sintered in the furnace. The sintering process determines the shade and 
mechanical properties of the restorations. However the problem with post-processing in the furnace is non-uniform shrinkage of the two dissimilar materials (Brick et al, 2004).

Although ceramics are aesthetically very attractive in dentistry (Strietzel, 2004), laser based SFF processes like SLM have not yet been able to produce parts which are accurate and have good mechanical properties. Porcelain-fused-to-metal restorations require a metal substructure which is then coated with porcelain. The metal part is made mostly from noble metals because of their better bio-compatibility, good mechanical properties and good metalceramic bonding. Base metal ( $\mathrm{Ni}-\mathrm{Cr}$ and $\mathrm{Co}-\mathrm{Cr}$ ) casting alloys are usually selected when a more economical metal unit is desired (Anusavice, 2003). The problem with base metals is their high melting temperatures, the possibility of oxidation during the casting process and high hardness which requires more time in the dental lab to post process it. Precious metals like gold have also been used for centuries for dental applications and porcelain-fused-tometal restorations (Figure 3-9). The requirements of metal alloys for porcelain-fused-to-metal restorations are discussed in detail below:

Figure 3-9: Cross section of a porcelain fused to gold crown (Cascone, 2003) 


\subsubsection{Biocompatibility}

As a dental prosthesis becomes part of the patient's mouth, it is extremely important for that part to be non-toxic to its host. Bio-compatible metals are already clearly identified and include both precious and non precious metals. Apart from biocompatibility, a patient can be allergic to a specific material. This depends upon the individual patient and the problem can be eradicated based on individual assessment.

\subsubsection{Tarnish and Corrosion Resistance}

For a material its tarnish and corrosion resistance is another factor which qualifies it to be used in dentistry. With noble metals this is not a matter of concern. Due to a variety of substances that can be present in the oral cavity, tarnish films can be deposited on the implant. A common example is the presence of a sulphide film on the gold-silver-copper alloy which are silver and copper sulfides. Sulfides are formed due to the presence of sulfur in various food items.

\subsubsection{Strength}

Strength of the alloy is another important factor and the alloy must remain in the elastic region for it to work properly. If any plastic deformation occurs, the part won't fit and it will cause great discomfort to the patient.

\subsubsection{Thermal Characteristics}

The melting range of the alloys affects the casting process and properties of the parts produced. The casting process requires the alloy to have its melting range in a limit in which it can be melted and casted with the available equipment. Also the meting temperature for the alloy should be high enough so that it does not melt during the porcelain baking cycle.

\subsubsection{Porcelain-to-Metal Bonding}

The bonding between porcelain and metal is also very important. With noble metals, bonding of porcelain to alloy is achieved by chemical bonding. This is achieved by removing the oxide films when the porcelain is cured during the baking cycle (Cascone, 2003). 


\subsubsection{Existing Manufacturing Systems in Dentistry}

Table 3-1 lists some of the commercially available systems. The type of restorations that these equipments can manufacture includes inlays and onlays. Inlays are dental restorations like a filling although they lie within the cusps and bumps of the chewing surface of the teeth. Onlays are larger in size than inlays and cover the actual chewing surface of the teeth.

\begin{tabular}{|c|c|c|}
\hline CAD/CAM systems & Restoration type & References \\
\hline CEREC (Sirona Dental GmbH) & $\begin{array}{l}\text { Inlays/ onlays } \\
\text { Crowns/ bridges }\end{array}$ & $\begin{array}{c}\text { (Sirona, 2009), } \\
\text { (Mormann, 2006) }\end{array}$ \\
\hline GN-1 (GC Corporation) & Inlays/ Crowns/ bridges & (GC Corporation, 2009) \\
\hline $\begin{array}{l}\text { Medifacturing (BEGO Medical } \\
\qquad \mathrm{GmbH})\end{array}$ & Dental frames & $(\mathrm{BEGO}, 2009)$ \\
\hline ZENO TEC & Crowns/ bridges & (WIELAND Dental, 2009) \\
\hline LAVA $^{\mathrm{TM}}$ (3M ESPE Dental AG) & Crowns/ bridges & (3M ESPE, 2009) \\
\hline $\begin{array}{c}\text { NobelProcera }^{\mathrm{TM}} \text { (Nobel Biocare } \\
\mathrm{AB})\end{array}$ & Crowns/bridges & (Nobel Biocare, 2009) \\
\hline
\end{tabular}

Table 3-1: $\quad$ Some of the commercially available CAD/ CAM systems in dentistry

\subsubsection{Additive Manufacturing in Dentistry}

Additive manufacturing technologies have also been used for dental applications. Additive manufacturing processes like 3D printing, Inkjet printing, SL and Selective Laser Sintering (SLS) could be used to produce master patterns, which can then be used for mould manufacturing. This mould can be used to cast the final part. Apart from RP processes, RM processes such as SLM can produce metallic crowns and bridges directly from powder metal. The CAD model of the dental restorations can be obtained by scanning and modelling in any commercially available software. This CAD model is sliced and sent to the SLM machine for production. The SLM process is more suitable for this application due to the complexity, low volume and high customization of the individual parts. The production of these unique restorations in one build considerably reduces the production costs. The German companies 
BEGO and EOSINT use laser sintering and melting technologies to produce crowns and bridges. Figure 3-10 shows some of the parts produced by BEGO and Sirona.

a

Figure 3-10: (a) SLM made cobalt-chromium bridges by the Medifacturing system from BEGO (BEGO, 2009) and (b) by infiDent system from Sirona (Sirona, 2009)

\subsection{Summary}

The introduction of digital methods in medicine and dentistry has changed the way manufacturing was conceived. Different digital scanning methods such as CT, MRI, Ultrasound imaging and 3D scanners are currently being extensively used in the medical field. RP and RM technologies are increasingly being used to utilise these medical imaging/scanning technologies to produce prototypes for better understanding of the problem or produce directly used implants for patients. The manufacturing of customised hearing aids, drill guides for complex spinal operation, cranioplasty plate and tri-flange implant for hips replacement are some of the examples of collaboration of RM technologies and the medical field. Similarly, the dental field has also been using the digitisation technologies to manufacturing complex and customized inlays and onlays. A number of contact and non-contact scanners and manufacturing setups are commercially available to perform the digitization and manufacturing of dental parts with ease and accuracy. Most of the dental crowns manufactured these days are porcelain-fused-to-metal restorations. These restorations have a metal substructure with porcelain coating on top, hence mimicking the natural tooth (harder form the outer and soft form the inner). The RM technologies such as Selective Laser Melting (SLM) could be used to manufacturing these highly customised dental restorations.

Based on the literature review presented in Chapter 2 and Chapter 3, the next chapter defines the research novelty and aim and objectives of this research work. 


\section{Chapter 4}

\section{HYPOTHESIS AND RESEARCH OBJECTIVES}

\subsection{Research Novelty}

The most commonly used biomaterials for the fabrication of dental crowns, inlays and onlays include dental ceramics, metal alloys and composites (porcelain-fused-to-metal). Porcelain-fused-to-metal restorations have been the choice for dental crowns because of their biocompatibility and aesthetics. In porcelain-fused-to-metal restorations the substructure is made from biocompatible metals (such as gold alloys) and porcelain is fused on top to give it a hard chewing surface. The use of high carat gold alloy has been the aim for dental purposes because of its high biocompatibility, aesthetics and ease of cold working, but the difficulties associated with casting 24 carat gold restricts its use. ARGEN $^{\odot}$ has introduced a new material called the 'Goldtech Bio 2000' which brings both biocompatibility and aesthetics together into the dental field. Goldtech Bio 2000 is a unique 24 carat castable alloy. Goldtech Bio 2000 consisting of 99.7 percent gold (24 carat gold alloy) with some addition of indium and zinc to achieve gold porcelain adhesion. This combination gives a superior alternative for aesthetic restorations with porcelain. This alloy is used to fabricate aesthetic porcelain fused to metal restorations. Because Goldtech Bio 2000 is 24 carat (99.7\%) gold alloy, it retains the true rich colour of pure gold which is the best background for developing warm porcelain shades. An alloy addition such as platinum, palladium or silver whitens the yellow colour rapidly. The indium and zinc trace elements produce an almost transparent oxide on the surface of the alloy which bonds it to porcelain (ARGEN, 2009).

The lost wax casting method has been used for decades for manufacturing dental crowns and bridges etc but the method involves too many processing steps. Laser based Rapid Manufacturing (RM) processes such as selective laser sintering (SLS), Laser Engineered Net Shaping (LENS ${ }^{\mathrm{TM}}$ ), Direct Light Fabrication (DLF), Direct Metal Laser Sintering (DMLS) and Selective Laser Melting (SLM) have induced flexibility into the manufacturing of complex 3D metallic parts. As compared to the sintering of powder in some other Solid Freeform Fabrication (SFF) processes, the complete melting of powder in the SLM process 
could produce parts with much higher density and strength. There has been a variety of academic work using SLM to process tool steel, stainless steel, iron, copper, titanium and nickel alloys (Abe et al, 2001), (Su, 2002), (Abe et al, 2003), (Kruth et al, 2004), (Pogson et al, 2004), (Santos et al, 2004), (Childs, 2005), (Mumtaz et al, 2006), (Yadroitsev et al, 2007). However, very little work has been published on the processing of precious metals and alloys including the presentation by Thorsson (2006). The manufacturing flexibility provided by the SLM process could be used to produce highly customized parts for jewellery and dental purposes etc with ease and eliminate constraints associated with the casting process.

This research work focuses on the SLM of 24 carat gold powder. Motivated and supported by ARGEN Corporation USA, this research focuses on the feasibility of using SLM for processing 24 carat gold alloy to produce a substructure for porcelain-fused-to-metal restorations. Gold is one of the most reflective metals and most reflectors use gold coating for better performance. The relative lack of published material and inherent difficulties in processing gold powder such as its high reflectivity made it a challenge for this research work.

\subsection{Research Aim and Objectives}

\subsubsection{Research Aim}

The aim of this research is to identify and understand the role of suitable processing parameters required for laser melting of 24 carat gold and to check its possible application in manufacturing dental crowns.

\subsubsection{Research Objectives}

A number of objectives set for this research work were:

1. Identify the radiation absorption properties of the gold powder and understand its effect on the powder compaction and particle size.

2. Characterize the gold powder for its shape, Particle Size Distribution (PSD), apparent density and tap density.

3. Establish a usable processing window for good melting of 24 carat gold powder and identify the suitable processing parameters.

4. Develop an understanding into the SLM of 24 carat gold powder. 
5. Check the effect of layer thickness and hatch distance on the internal porosity of the parts.

\subsection{Research Methodology}

The research methodology employed to material qualification for SLM process, processing and analysis which are explained in detail below:

\subsubsection{Material Selection and Characterisation}

24 carat gold powder of mean particle size around $25 \mu \mathrm{m}$ was selected for this purpose. Gold powder was provided by $\mathrm{ARGEN}^{\circledR}$ Coorporation USA. A smaller particle size powder was selected to manufacture thin dental structures, which is explained in detail in Chapter 8. Gold powder was analysed for Particle Size Distribution (PSD), apparent density and tap density.

\subsubsection{Processing}

Processing was divided into three different sections: single beads, single layers and then multiple layer manufacturing.

\subsubsection{Experimental setup}

An SLM 100 machine was used for processing gold powder. This machine has a $\phi 125$ x $80 \mathrm{~mm}$ build volume. It uses a $50 \mathrm{~W}$ continuous wave fiber laser with spot size of around $35 \mu \mathrm{m}$. The small spot size and smaller build area made this system an ideal choice for this work.

\subsubsection{Identifying suitable processing parameters}

Experiments were first conducted for single beads on the substrate to check the influence of laser power and scan speed on the processing of gold powder. These parameters were varied in their full operating range. This was helpful in establishing the effective processing window. Single layers of gold were produced after identifying the suitable processing parameters. 
Finally, based on the findings from single bead and single layers, multiple layers $4 \mathrm{~mm}$ cube samples and dental crowns were to be produced from the best parameters for further analysis

\subsubsection{Analysis}

The $4 \times 4 \times 4 \mathrm{~mm}$ gold cubes produced from the suitable parameters obtained in the previous section were analysed either by the Archimedes method or optical imaging method to check for the internal porosity in these parts. Other parameters such as hatch distance and layer thickness etc were changed to reduce the part's internal porosity. Experiments were performed to check whether a pre-scan technique could be used as a powder bed preheating technique. Mechanical properties such as hardness and modulus of the cubes were checked using microhardness and nanoindentation methods. 


\section{Chapter 5}

\section{LASER MATERIAL INTERACTION}

\subsection{Radiation Absorption of Metal Powders}

As discussed earlier in section 2.3.3.1, when the radiation is incident on a levelled powder surface, one portion of the radiation strikes the particles lying directly on the surface, while another portion penetrates the pores and reaches the interior of the powder (Figure 5-1). This radiation is repeatedly reflected from particles located at deeper levels in the powder. These particles, functioning as pore-sinks, act as blackbodies that intensively absorb radiation (Tolochko et al, 1997). The particles of several surface monolayers undergo heating by the laser radiation. Subsequent propagation of heat from the initially heated particles occurs by normal heat conduction and some heat is transferred to the environment. Figure 5-1 shows this phenomenon of radiation-material interaction where $I_{o}$ is the incident radiation, $Q_{c}$ is the heat transfer through conduction and $\mathrm{I}_{\mathrm{r}}$ is the reflected radiations.

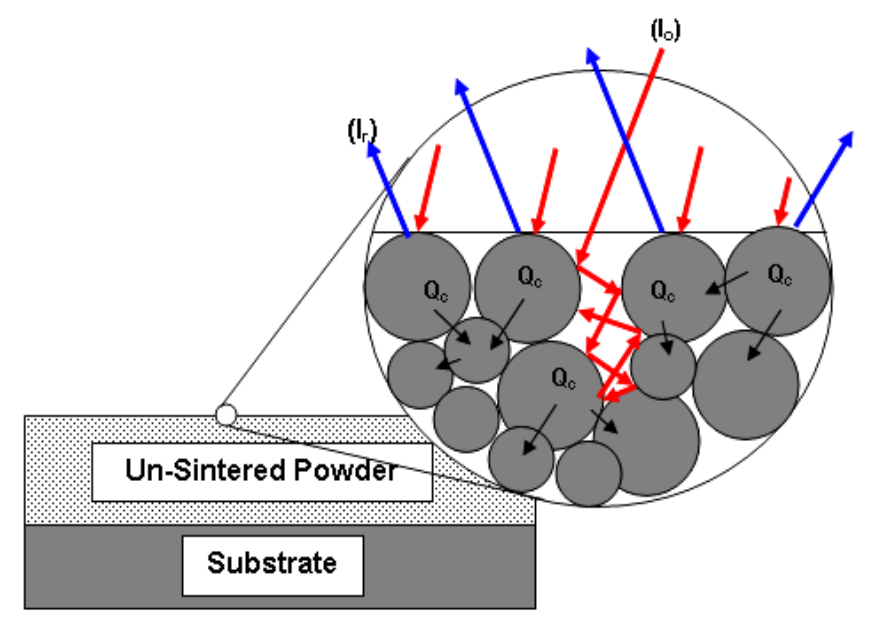

Figure 5-1: Radiation absorption and reflection from a powder bed 
Figure 5-2: Different components of reflected radiations from the metal powders

Special Diffuse Reflectance Infrared Fourier Transform Spectroscopy (DRIFTS) equipment is needed to measure radiation absorption of the powder material. The mechanics of radiation absorption are discussed below to explain how DRIFTS works.

The reflection of radiations (Ir) from a powdered surface occurs in three different ways, namely; specular, specular diffuse and true diffuse reflectance as shown in Figure 5-2. Many factors govern the amount of each type of reflection from the powder such as particle size, compaction density, absorptivity and refractive index etc. The amount of reflected radiation presented in this section is measured by a DRIFTS accessory called the 'Specac' (Selector Diffuse Reflectance Accessory) as shown in Figure 5-3.

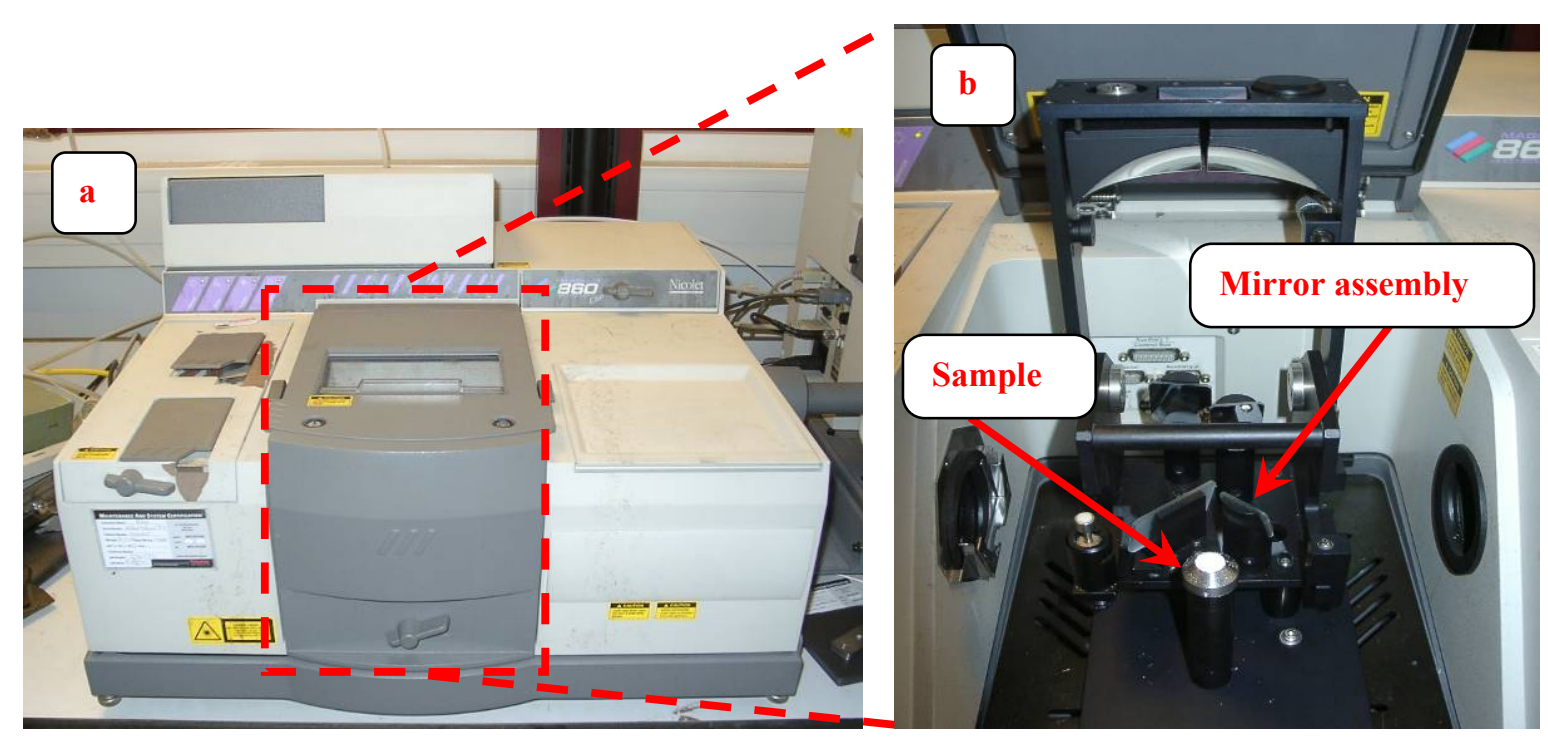

Figure 5-3: (a) DRIFTS equipment with (b) Selector diffuse reflectance accessory 
This accessory has a mirror configuration, which is designed to optimise the collection of diffuse reflection, which also contains the specular component of the sample under investigation. An alternative configuration is that shown by Tolochko et al. (1997) in which the detector is off-line to the incident radiation.

Five different samples were tested for their reflectance in the near IR range of 10601090nm wavelength. The materials selected were Al $12 \mathrm{Si}, \mathrm{Cu}$, Gold, H13 Tool Steel and Stainless Steel powders. Reflectance spectra were collected using a white light source. An Mercury-Cadmium-Telluride (MCT) detector and a quartz beam splitter were selected to obtain data in the near-infrared region in which the fiber laser of the SLM 100 system operates (1060-1090nm wave length). As other factors like type of material, particle size and compaction had a major influence on the reflectivity of the powder material, so the difference between the source (white light source or laser source), both operating in the same IR range, was considered to be almost negligible (Coupland, 2009).

\subsubsection{Sample Preparation}

The sample under investigation was compacted in a cup (10mm diameter and $5 \mathrm{~mm}$ in depth) and levelled using a glass microscope slide ensuring that the top surface was as flat as possible. Zinc oxide $(\mathrm{ZnO})$ was used as the reference background which is known to have reflectivity close to unity (Tolochko et al, 1997). The results obtained from different powder samples were normalised with the results from $\mathrm{ZnO}$ to get actual material reflectance.

\subsubsection{Results and Discussions}

Figure 5-4 shows the percentage reflectance of the sample powders. Gold powder was by far the most reflective metal powder with reflectance of about $85 \%$. The high reflectivity of gold is also true for gold in bulk. Although gold can only reflect about $45 \%$ of the visible light, its reflectivity is increased to about $99 \%$ (pure electroplated gold) in the IR range and this is the reason for its extensive use as a coating material in IR reflectors. However, in powder form its reflectivity drops to about $85 \%$ (as shown in Figure 5-4). This reduction in the reflectivity of gold in powder form makes it processable even with an IR radiation source. In this research work an IR fiber laser (YLR-50-SM) was used for processing 24 carat gold powder for which a detailed description is given in Chapter 7 . 


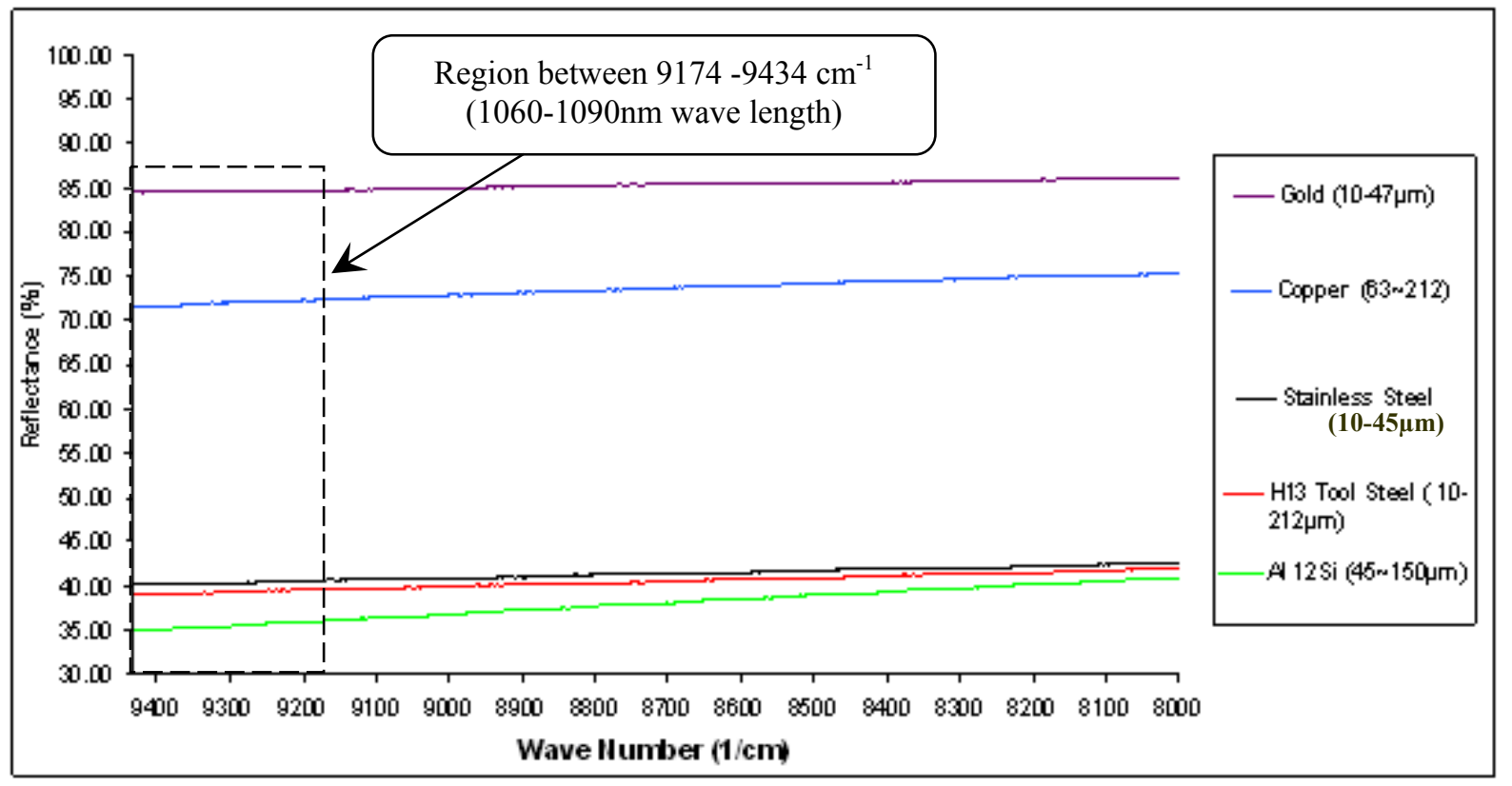

Figure 5-4: Percentage reflectance of different metal powders

From Figure 5-4, Al12Si had the lowest reflectance due to the addition of 12 percent $\mathrm{Si}$ in the Aluminium powder, while there was very little difference (approximately $1 \%$ ) between the reflectance of H13 tool steel and stainless steel powders throughout the 1060-1090nm wavelength range. Copper was found to be the second most reflective metal of the five sample materials with a reflectance of $71 \sim 72 \%$.

The effect of powder particle size and powder compaction on the reflectance of a powder layer is discussed further in this section.

\subsubsection{Effect of Particle Size on Radiation Absorption}

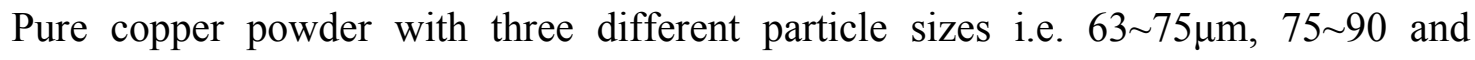
90 212 was tested for absorption. All the samples were levelled in the same manner and each of the experiments was repeated three times for repeatability. Figure 5-5 shows the difference in absorption (average of three readings) of different particle size copper powders. It is evident from Figure 5-5 that particle size has an effect on the absorption of radiation of the material. A smaller particle size tends to have less reflection and thus higher absorption. The higher absorption of smaller particles is related to higher surface area to volume ratio of the particles and the possibility of many very small gaps between these small particles. These smaller gaps would act as a black body and radiation is absorbed by multiple absorption and reflections. 
Because of the smaller size, radiations once enter these smaller gaps and cannot escape easily compared to larger gaps between larger particles. Although the difference between the reflectance with powder size is not that high (2.5\% in Figure $5-5)$ it could be useful in processing highly reflective metals like gold, silver and aluminium etc.

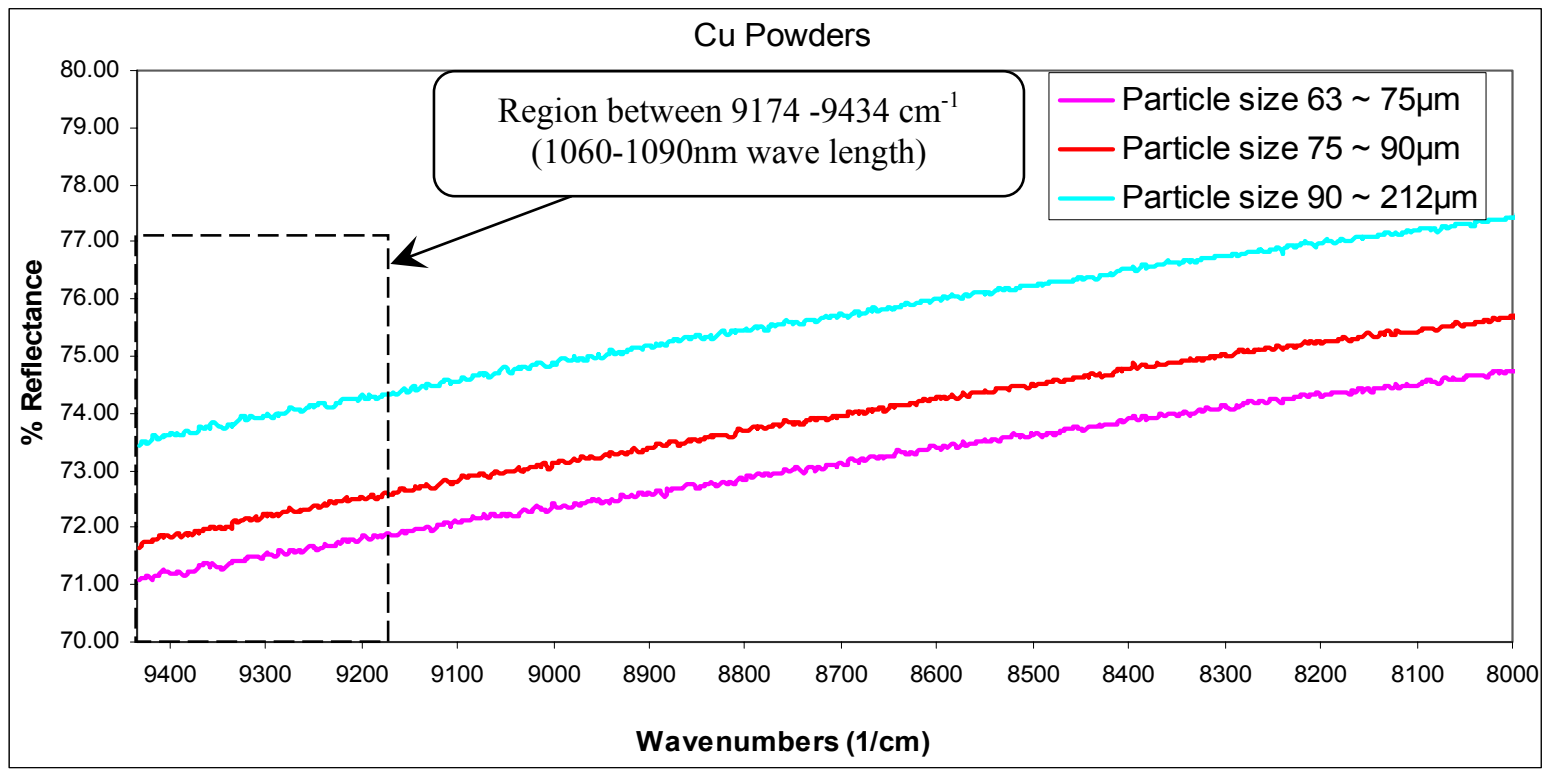

Figure 5-5: Reflectance of copper powder with different particle sizes

\subsubsection{Effect of Particles Packing on Radiation Absorption}

Particle packing density (or green density) of the powder layers in a layer-by-layer RM process has a positive influence on the final density of the parts (Zhu et al, 2007). In order to check the effect of powder bed density on the radiation absorption, 24 carat gold powder was used. Gold powder was packed into the sample cup with two packing densities i.e. apparent density of $9.5 \mathrm{~g} / \mathrm{cm}^{3}$ and maximum tap density of $10.4 \mathrm{~g} / \mathrm{cm}^{3}$. The detail of apparent and tap density of gold powder is given in Chapter 6. This gave an idea of reflectance of powder in the most loose state of apparent density and the maximum packed state of maximum tap density. Figure 5-6 show that gold powder with lower packing density absorbs more radiation (approximately 3\%) as compared to the densely packed particles. This is in accordance with the earlier assumption of radiation absorption that with loosely packed powders there would be more gaps and thus greater chance of radiation going in these gaps. As stated earlier, these gaps acts like black bodies and thus more radiation is absorbed in the powder bed by multiple absorption and reflections. In densely packed particles, radiations are only absorbed by the particle lying on the top surface of the powder bed. Therefore, for reflective metals a smaller 
particle size combined with low packing density would absorb a higher percentage of incident radiation which could help in the processing of these metals.

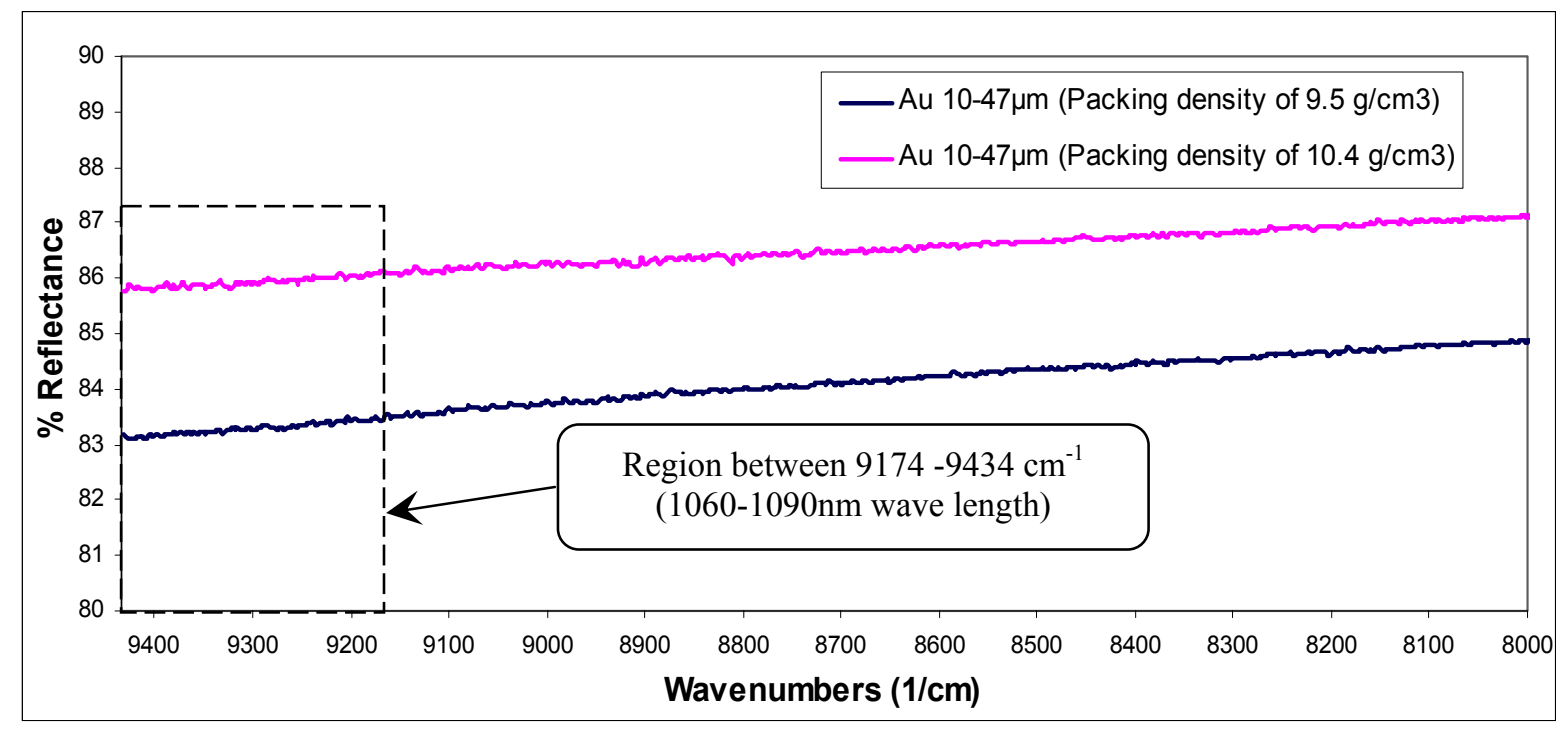

Figure 5-6: Reflectance of gold powder packed with different densities

\subsection{Summary}

This chapter presented a brief overview of the interaction of incident IR radiation on the powder layer of different metals. It was explained that the interaction of radiation on a powder layer is quite different than the material in bulk. The DRIFTS accessory was used for measuring reflectance of the sample metal powders. Gold powder, which is the main material under consideration in this research work, was found to have very high reflectance (around $85 \%$ ) as compared to other materials like copper, $\mathrm{Al} 12 \mathrm{Si}, \mathrm{H} 13$ tool steel and stainless steel powders. Gold is considered to have a very high reflectance in coatings and the polished form (around 99\% in IR range) but in powder form its reflectance reduced (absorption increased) which is the reason it could be processed with an IR fiber laser (wavelength 1060-1090nm) (see Chapter 8).

Different factors affect absorption or reflectance of the powder layer such as material type, powder compaction, particle size etc. A copper powder layer with smaller particle size absorbed a higher percentage of radiation as compared to larger particle powders with the same compaction. Powder compaction was found to have an inverse effect on the reflectance, where higher packing density of gold powder absorbed less radiation than loosely packed powder layer because of fewer gaps between the metal particles. The results obtained in this 
chapter were very useful in understanding the difference in the absorption of a material in powder and bulk form.

The following chapter explains in detail other powder material properties such as particle shape and size distribution, apparent density, tap density and moisture absorption. 


\section{Chapter 6}

\section{CHARACTERISTICS OF THE POWDER}

\subsection{Introduction}

The density of powder material is not a single independent property rather it depends on quite a few factors such as particle shape, particle size distribution, the percentage of smaller and larger particles in the powder system, powder surface free energy, humidity, friction between the powder particles, friction with the container walls, presence of antistatic agents, agglomeration (German, 1989), height and type (through orifice or sieve) of fall and shielding (Santomaso et al, 2003). The surface free energy is the excess energy at the surface of the material as compared to bulk of the material. For material in liquid state, the surface free energy is the surface tension of the material which is the driving force behind sintering/fusion during laser processing. The surface free energy or interfacial energy $\gamma$ is defined as the change in the free energy $\mathrm{F}$ with area $\mathrm{A}$ at constant temperature, pressure and composition (as shown in equation (6.1)).

----- (6.1) (German, 1989)

The weak surface forces such as Van Der Waals attractions, electrostatic charges and magnetic forces influence very small particles of size below 0.05microns (German, 1989). As the gold powder size used in this research is large than 0.05 mocrons, therefore, the weak forces are not an issue for this research work.

The bulk density of powder is its mass divided by the volume occupied by the powder in its loose state. The volume includes the space between particles as well as the volume occupied by the powder particles as a whole (Svarovsky, 1987). It is also referred to as the mass of the powder that can be packed into a specific volume (Abdullah and Geldart, 1999). Different bulk density parameters in order of their increasing value are aerated bulk density, poured bulk density (apparent density), tap density and compacted bulk density (Svarovsky, 1987). 
Abdullah and Geldart (1999) define aerated bulk density as the density of dispersed powder settled in a container under the influence of gravity. Svarovsky (1987) defined aerated bulk density as the density measured when particles are separated from each other by a thin film of air and they are not in direct contact with each other. However, it is also defined as the density when powder is aerated and allowed to settle gently such that it is in the most loosely packed condition.

Poured bulk density also known as the apparent density can be defined as the density of powder poured into a fixed container from a specific height. It is probably the most widely measured bulk density property of the powder. Poured bulk density for metallic powders is covered under the ASTM B212 (ASTM B212, 1999), ASTM B329 (ASTM B329, 1998), BS EN 23923-1(BS EN 23923-1:1993), BS EN 23923-2 (BS EN 23923-2, 1993), ISO 3923-1 (ISO 3923-1:1979) and ISO 3923-2 (ISO 3923-2, 1981) standards.

Tap density is the bulk density obtained after compaction by tapping or vibrating the container following a specified procedure. Tap density can be performed both manually and automatically. In the manual method, powder is poured into the graduated glass cylinder which is tapped against a hard surface form a fixed height until maximum compaction is achieved. In the automated method, powder is poured into the graduated glass cylinders and tapped automatically with a certain frequency of vibration and tapping from a specified height.

\subsection{Powder properties}

The material used was 24 carat $\mathrm{ARGEN}^{\complement}$ (The ARGEN corporation, 2009) gold (Au) powder. This section presents powder properties such as particle size distribution, apparent density and tap density measurements of the gold $(\mathrm{Au})$ powder.

\subsubsection{Particle Size Distribution (PSD) analysis}

Particle Size Distribution (PSD) of the gold powder was carried out with the laser diffraction technique using a Mastersizer 2000 (Malvern Instruments, 2009). The laser diffraction technique needs powder to be dispersed in an aqueous or non-aqueous solution. Here, gold powder was first dispersed in water but due to poor dispersion of gold powder in water, it was then dispersed in isopropanol. Figure 6-1 and 6-2 (full report is presented in Appendix A) shows the PSD obtained using water and isopropanol as dispersants. The PSD results obtained using water as a dispersant showed the smallest particle size of around $5 \mu \mathrm{m}$ 
whereas the smallest particle size obtained with isopropanol as a dispersant is as low as $3.3 \mu \mathrm{m}$. For confirmation of the results, PSD was performed again using isopropanol, which also gave the same results. This difference in the two particle sizes was attributed to the fact that gold powder does not disperse well in water as compared to isopropanol. This was evident from the visual observation of both these solutions, where the colour of isopropanol changed to mild yellow on addition of gold powder, where no such observation was made in water and gold powder was floating on top.

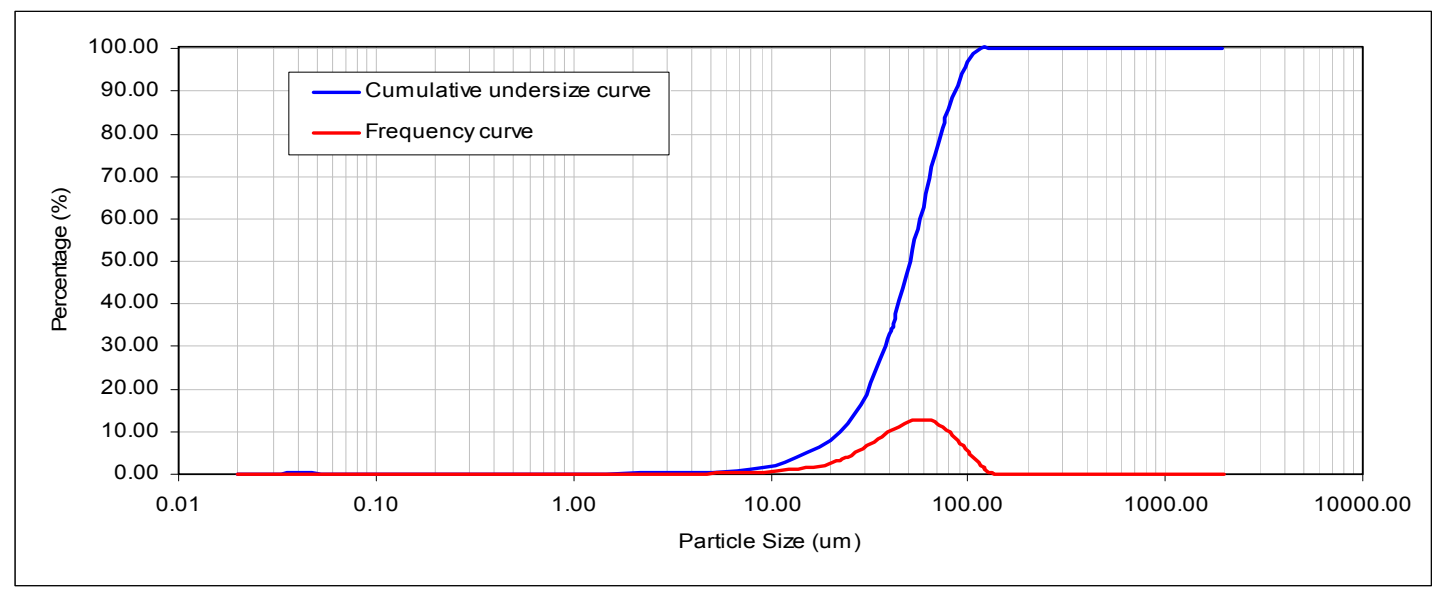

Figure 6-1: $\quad$ PSD of gold $(\mathrm{Au})$ powder in water: $\mathrm{d}(0.1)=23.163, \mathrm{~d}(0.5)=51.296$ and $\mathrm{d}(0.9)$ $=88.184$

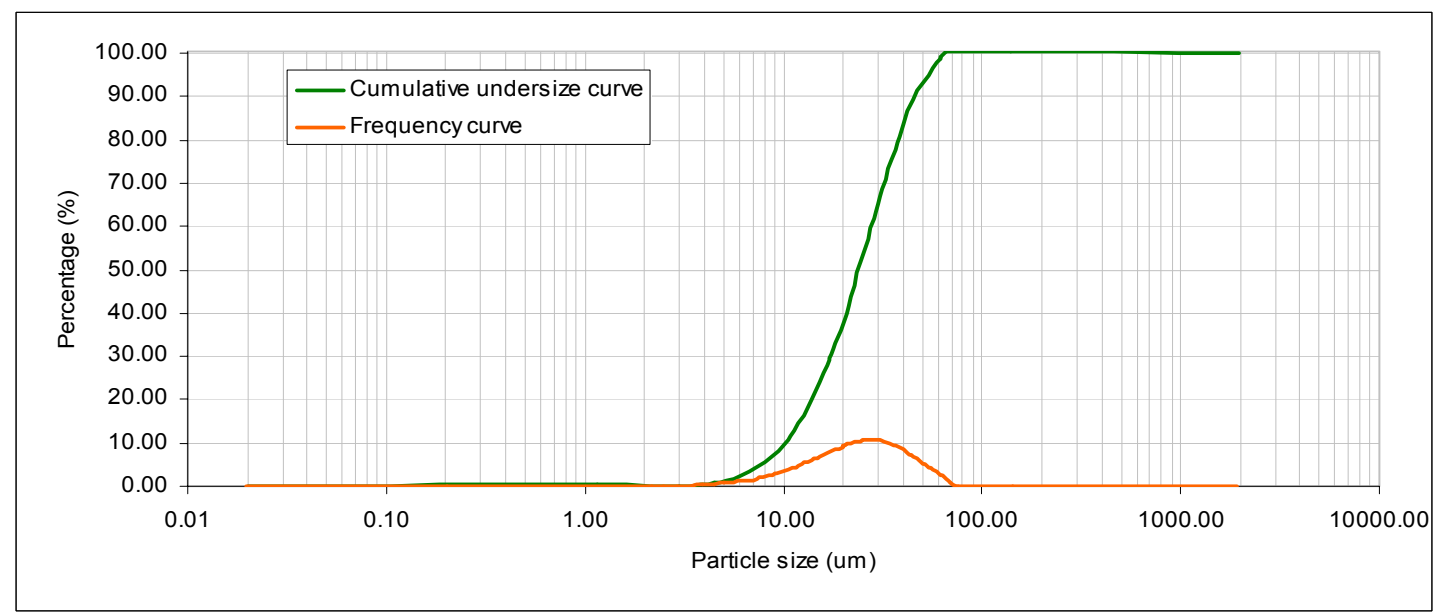

Figure 6-2: $\quad$ PSD of gold $(\mathrm{Au})$ powder in isopropanol: $\mathrm{d}(0.1)=10.43, \mathrm{~d}(0.5)=24.55$ and

$$
\mathrm{d}(0.9)=46.73
$$

In order to authenticate the results obtained from isopropanol, a Scanning Electron Microscope (SEM) was used. The images obtained from SEM (Figure 6-3) obtained for gold 
powder also suggests the smallest particles size of around $3 \mu \mathrm{m}$, which supports the observation of the non-dispersing nature of gold powder in water. The reason behind the different results of gold powder in water as dispersant can be explained by Figure 6-3c (marked in circle), which shows smaller satellite particles sticking to the larger particles forming agglomerates. When this agglomerated gold powder was added to water in the PSD analysis, it was dispersed very little hence giving a maximum particle size of around $88 \mu \mathrm{m}$ and mean particle size of $51.296 \mu \mathrm{m}$. The same gold powder when added to isopropanol for PSD analysis was dispersed well and indicated a maximum particle size of around $46 \mu \mathrm{m}$ and mean particle size of $24.55 \mu \mathrm{m}$. The SEM images taken at different points in the gold sample did not show any particles of size around $85 \mu \mathrm{m}$ but particles around $45 \mu \mathrm{m}$ size were abundant. Therefore it was confirmed that water is not a recommended dispersant for PSD analysis of gold powder and that PSD obtained with isopropanol gives more accurate readings.

The increase in the mean particle size has a great influence on the arrangement of particles as they try to form the dense packing. A larger mean particle size corresponds to a smaller percentage of finer particles. During tapping or agitation of powder for compaction, smaller and larger particles re-arrange themselves to form dense packing. Increasing the percentage of larger particles provide more inter-particle space for smaller particles to fill in. Most of the smaller particles tend to go downward to the bottom of the density cup hence leaving more voids in the middle and upper section of the cup (Abdullah and Geldart, 1999).

From Fig 6-3, the gold ( $\mathrm{Au}$ ) powder was found to be mostly spherical in shape with smaller satellite particles agglomerated to the larger particles. 

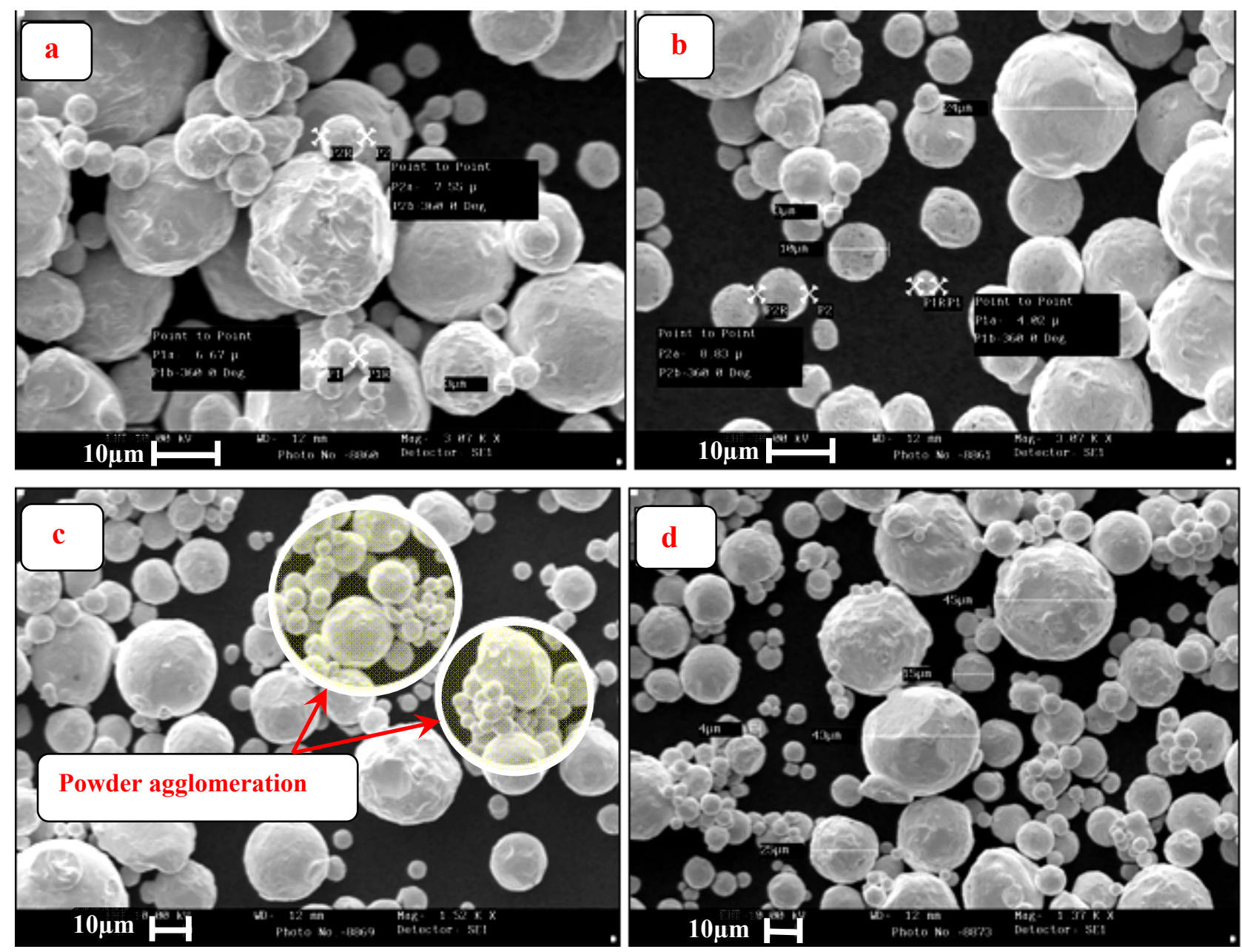

Figure 6-3: (a-d) SEM Images of ARGENC gold $(\mathrm{Au})$ powder

\subsubsection{Apparent Density}

The apparent density of powder is that in its loose state in a fixed size container. The powder's apparent density (or green density) is of great importance in layer-based Rapid Manufacturing (RM) processes. Zhu et al (2007) proved that apparent density of the powder bed directly influences final density of the sintered parts. The higher the density of powder bed, then the higher will be the density of final sintered parts. It is also known that the pouring height (shielded and non-shielded) of the powder also influences its apparent density measurements (Santomaso et al, 2003).

Gold powder was initially tested for apparent density according to ASTM B212-99, (ASTM B212, 1999), BS EN 23923-1:1993 (BS EN 23923-1:1993) and ISO 3923-1:1979 (ISO 3923-1:1979) standards. These standards use a Hall flowmeter funnel (Figure 6-4) and Carney Funnel (Figure 6-5) with an orifice of 2.5 and $5 \mathrm{~mm}$ respectively (depending on the 
Flowability of metal powder through 2.5 or $5 \mathrm{~mm}$ orifice). It was found that gold powder did not flow freely though the Hall flowmeter funnel or the Carney funnel. Even with the assistance of a metallic wire through the funnel, gold powder was very reluctant to flow and would stop flowing as the metallic wire stopped moving or was removed. Therefore, ASTM B329 (ASTM B329, 1998), BS EN 23923-2 (BS EN 23923-2, 1993) and ISO 3923-2 (ISO 3923-2, 1981) standards were adopted. These standards specify the apparent density measurement method for powder not flowing freely through a 2.5 or $5 \mathrm{~mm}$ orifice funnels. The equipment used for measuring apparent density of gold powder was the Scott Volumeter (Scott, 1960) (as shown in Figure 6-6). Here, apparent density was measured with the standard $25 \mathrm{~cm}^{3}$ density cup and two other cups with different height-to-diameter aspect ratios. These cups were used to check the variation in apparent density with change in the dimensions of the density cups.

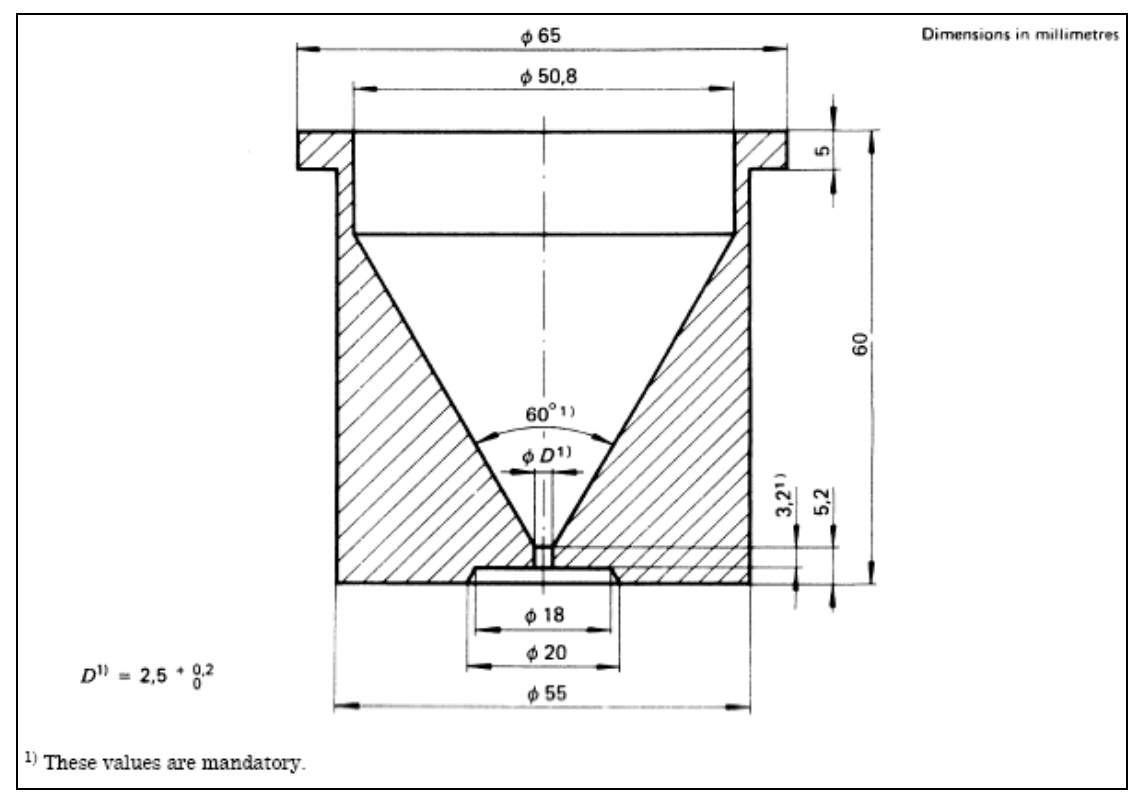

Figure 6-4: Hall flowmeter funnel 


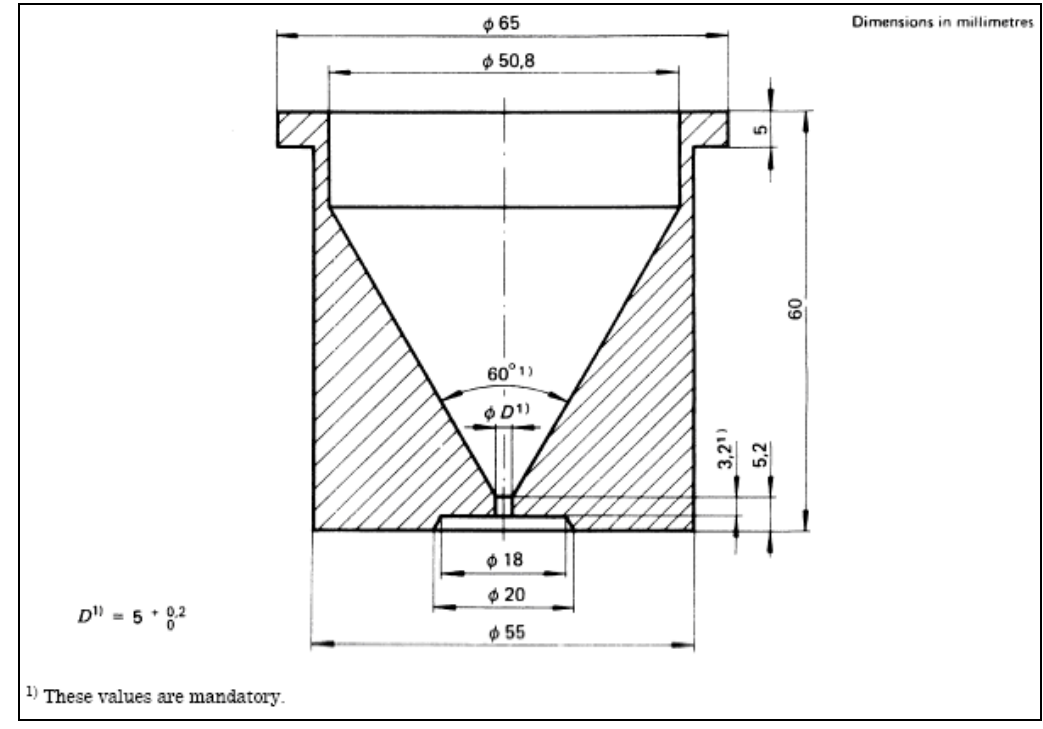

Figure 6-5: Carney funnel

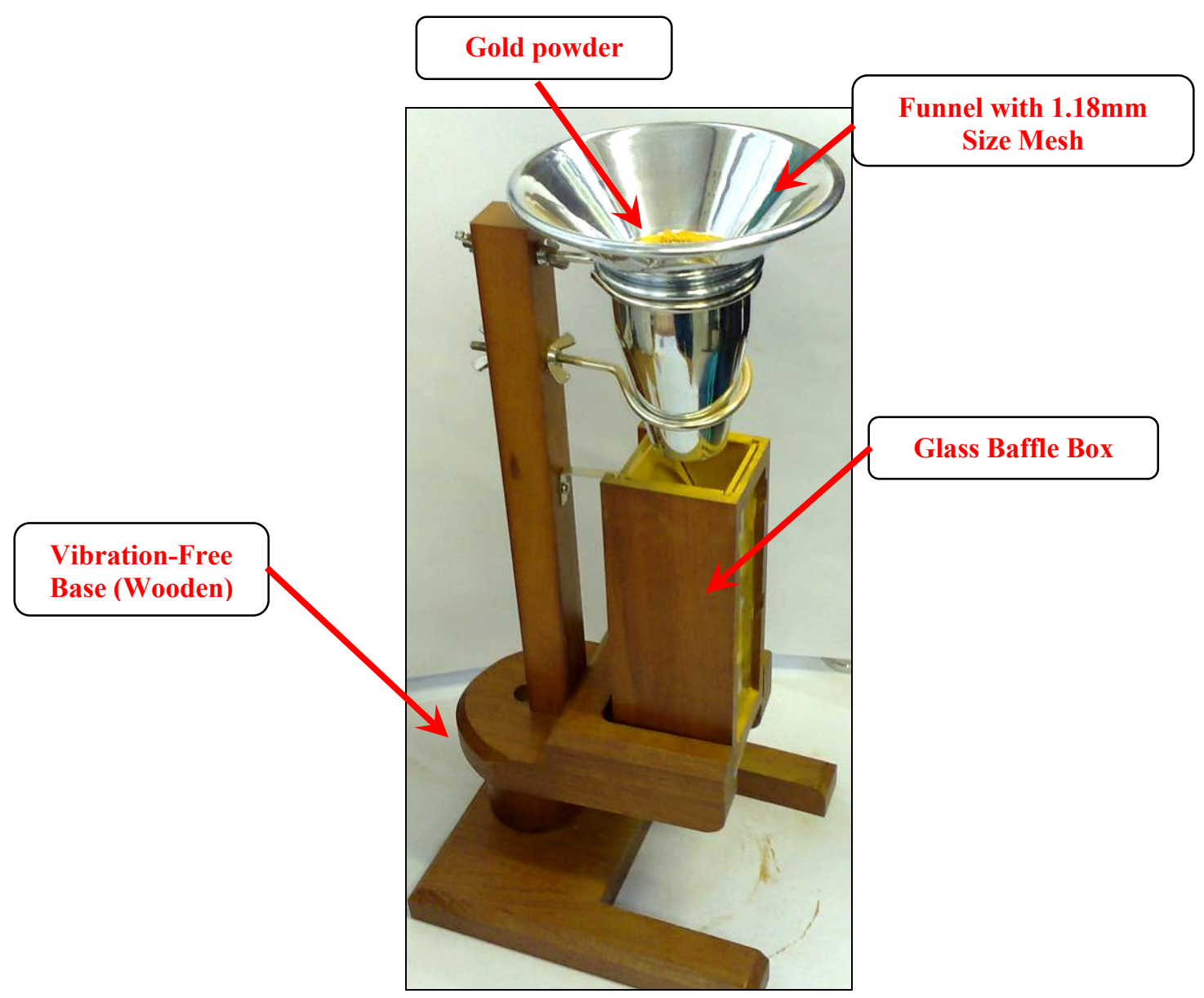

Figure 6-6: Scott volumeter 
With the standard method, a $25 \mathrm{~cm}^{3}$ brass density cup was first weighed on a digital balance which was capable of measuring to an accuracy of $\pm 0.05 \mathrm{~g}$ (as per the requirements of the standards). Gold powder was then added to the top hopper of the Scott volumeter and with the use of a nylon brush gold powder was assisted to flow through a $1.18 \mathrm{~mm}$ mesh size sieve. Gold powder flowed through the volumeter with ease onto the baffle glasses one by one and was collected in the $25 \mathrm{~cm}^{3}$ density cup at the bottom. A glass slab was used to scrape the extra powder from the top of the $25 \mathrm{~cm}^{3}$ density cup as carefully as possible so as not to disturb the cup and weighed on the digital balance. The weight of the sample was calculated from the difference of the two readings (with and without metal powder). Apparent density was calculated according to equation (6.1):

$$
\boldsymbol{\rho}_{\mathbf{a}}=\mathbf{W}(\mathrm{g}) / 25\left(\mathrm{~cm}^{3}\right)
$$

Where, $\rho_{\mathrm{a}}$ is apparent density of metal powder.

Apparent density experiments were also conducted with two stainless steel cups with different height-to-diameter aspect ratio. Figure 6-7 shows density cups with different heightto-diameter aspect ratio including the standard $25 \mathrm{~cm}^{3}$ density cup. Table $6-1$ specifies the aspect ratio of all the density cups used to measure apparent density of the gold powder including the standard $25 \mathrm{~cm}^{3}$ brass density cup. Figure $6-8$ shows variation in the apparent density for different aspect ratio density cups. It was noted that these variations in the cup dimensions (height and diameter) had a negligible effect on the apparent density of gold powder.

\begin{tabular}{|c|c|c|c|}
\hline S/NO & Aspect Ratio & Description & $\begin{array}{c}\text { Volume } \\
\left(\mathrm{cm}^{3}\right)\end{array}$ \\
\hline Cup \# 1 & 0.5 & Stainless Steel Density Cup & 10 \\
\hline $\begin{array}{c}\text { Cup \# 2 } \\
\text { (standard cup) }\end{array}$ & 1.5 & Standard Brass Density Cup & 25 \\
\hline Cup \# 3 & 2 & Stainless Steel Density Cup & 9 \\
\hline
\end{tabular}

Table 6-1: Aspect ratio of density cups 


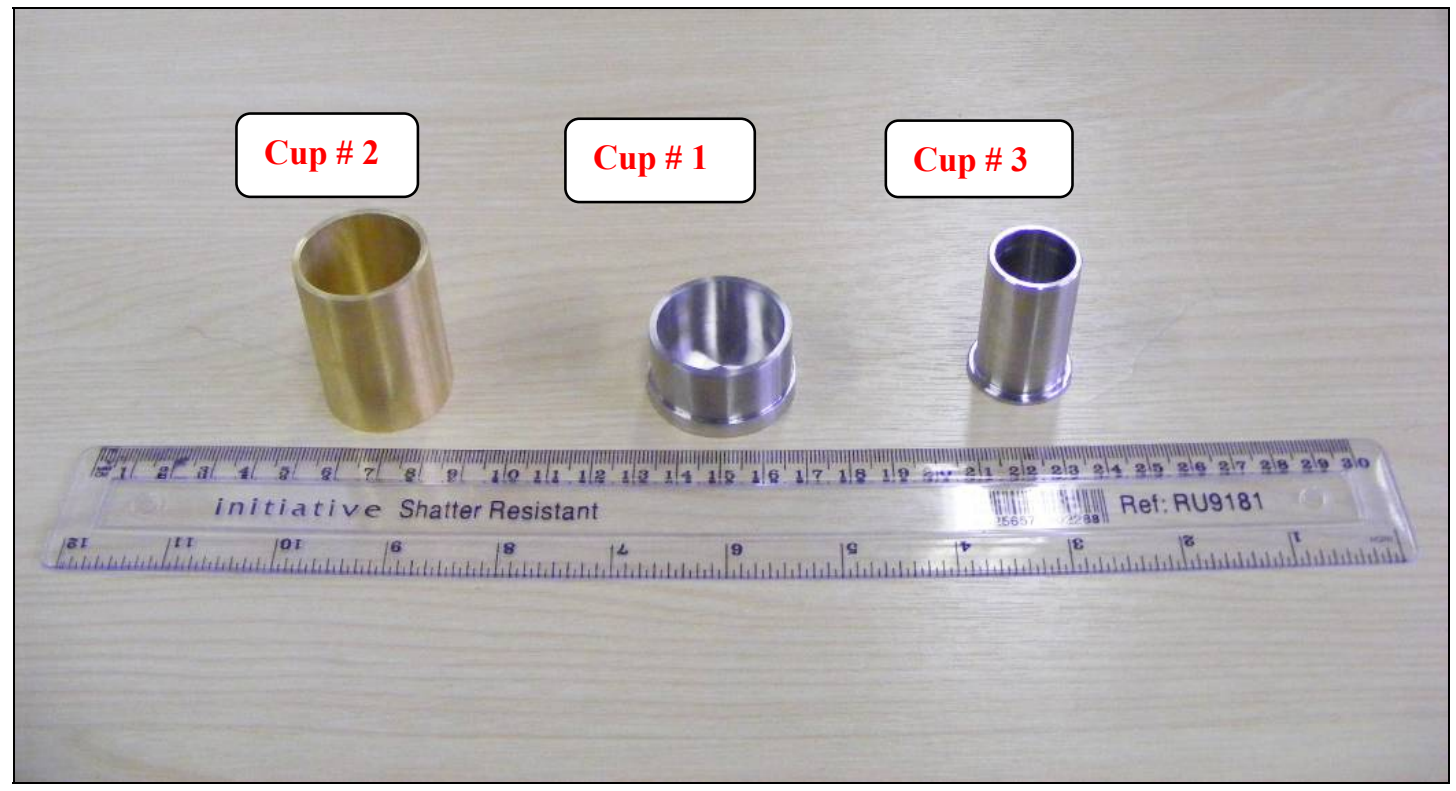

Figure 6-7: Apparent density cups with different aspect ratios

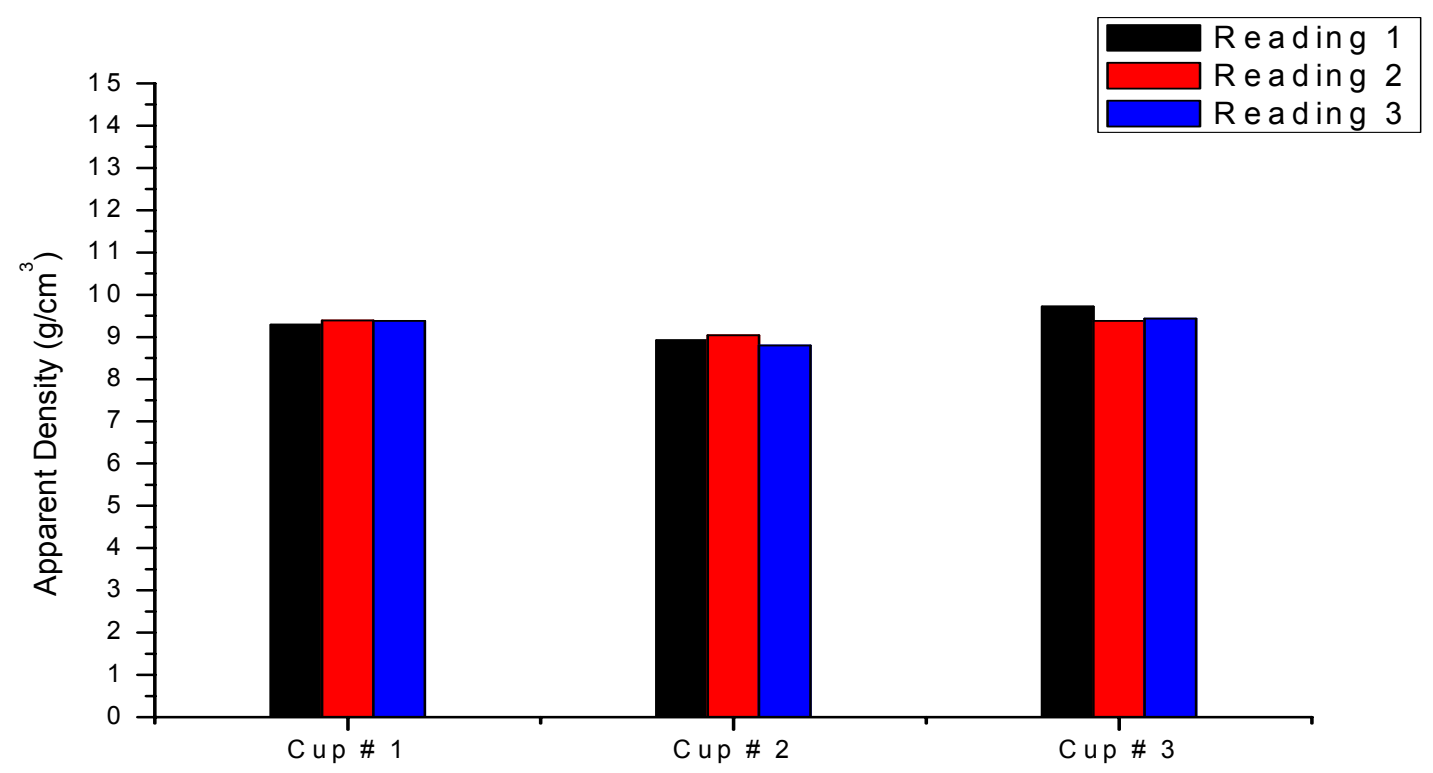

Figure 6-8: Apparent density with different aspect ratio density cups

\subsubsection{Tap Density}

The tap density of gold powder was determined by two different methods i.e. Constant Weight Tap Density (CWTD) method and Constant Volume Tap Density (CVTD) method. These two methods are explained as follows: 


\subsubsection{Constant Weight Tap Density (CWTD) Method}

The CWTD method is according to the ASTM B527 (2006) and BS EN ISO 3953 (1995) standards where tapping was performed by a manual method. As the apparent density of gold powder was found to be greater than $7 \mathrm{~g} / \mathrm{cm}^{3}$, so according to tap density standards for metallic powders, the mass of the gold sample needed was $100 \mathrm{~g}$. A $100 \mathrm{~g}$ sample of gold powder was weighed on a digital balance (accuracy $\pm 0.05 \mathrm{~g}$ ) and poured gently into the standard $25 \mathrm{~cm}^{3}$ graduated glass cylinder (as shown in Figure 6-9) to avoid any compaction of powder before tapping. It was also made sure that powder formed a horizontal level in the cylinder so that it could be easily measured. A total of 200 taps were performed for each sample with the manual method (according to BS EN ISO 3953:1995) using a hard rubber pad. The volume of the samples was measured directly from the readings on the standard $25 \mathrm{~cm}^{3}$ graduated cylinder (taken as average of three readings from the level of powder in the cylinder) to give the tap density after a specific number of taps. If the powder was not levelled in the cylinder then the average of the maximum and minimum value was taken as the tap density reading. Tap density after a specific number of taps was calculated according to equation (6.2). Each of the experiments was performed three times to check repeatability of the results.

$$
\boldsymbol{\rho} \mathbf{t}_{\mathbf{n}}\left(\mathrm{g} / \mathrm{cm}^{3}\right)=\mathbf{W}(\mathrm{g}) / \mathbf{V}_{\mathbf{n}}\left(\mathrm{cm}^{3}\right)
$$

Where, $\rho t_{n}$ is the tap density after $n$ number of taps, $W$ is weight of the sample ( $100 \mathrm{~g}$ for constant weight method) and $V_{n}$ is the volume occupied by the sample in the standard $25 \mathrm{~cm}^{3}$ graduated glass cylinder after $\mathrm{n}$ number of taps.

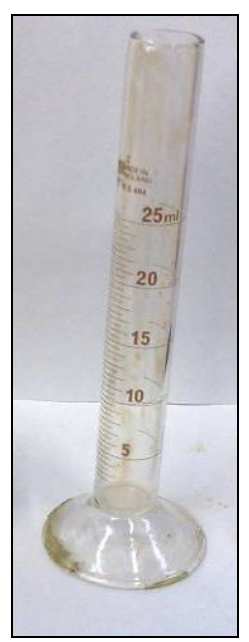

Figure 6-9: $\quad$ Standard $25 \mathrm{~cm} 3$ graduated glass cylinder 
Figure 6-10 shows the increase in tap density of gold powder with increasing number of taps for a standard $25 \mathrm{~cm}^{3}$ graduated glass cylinder. It can be noted that after 60 taps gold powder was almost completely compacted thereby reaching its maximum tap density. There was found to be very little change in the tap density after 60 taps. Figure 6-10 follows a typical tap density curve where powder is compacted quickly in the initial taps while less change is observed after it reaches its maximum compaction.

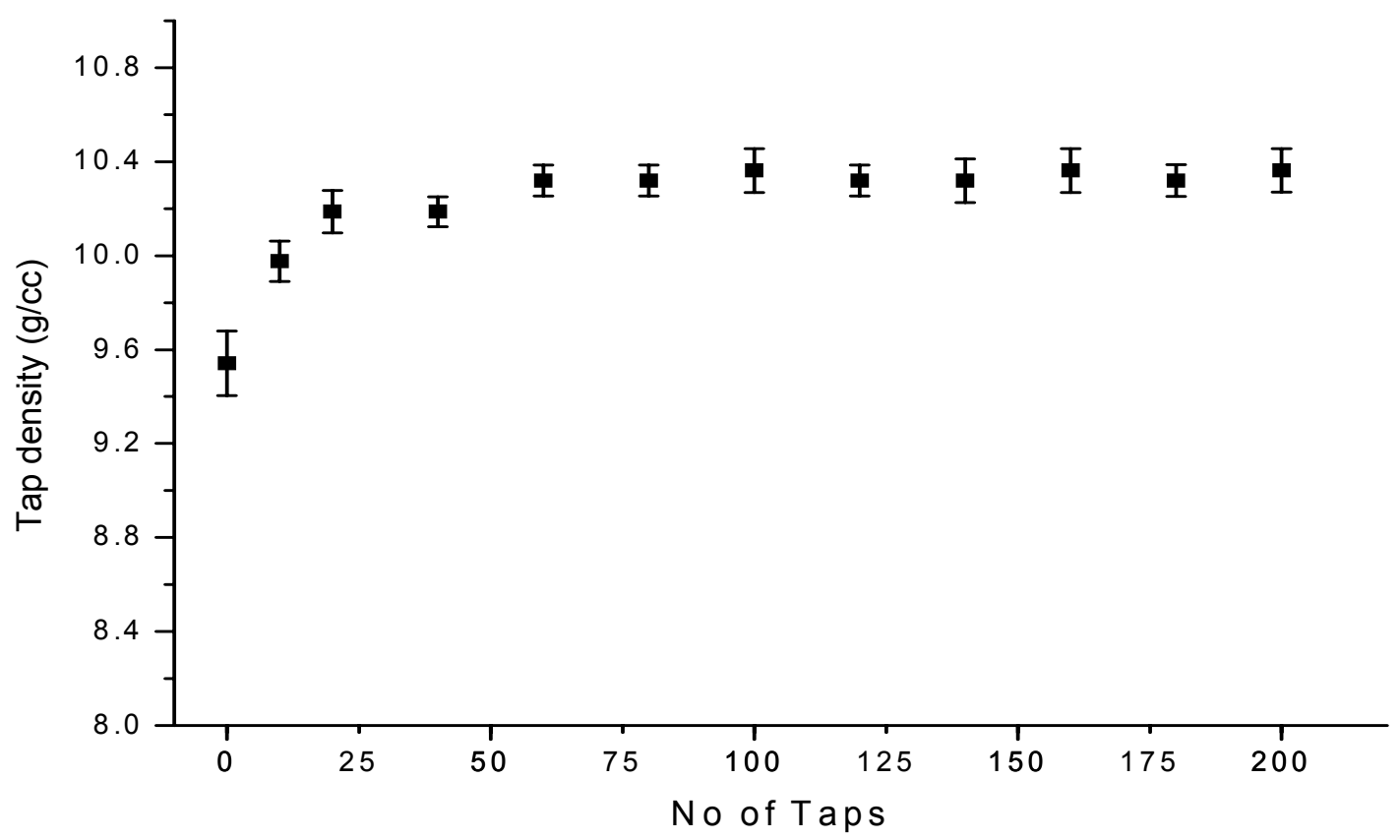

Figure 6-10: Tap density of gold powder with CWTD method

\subsubsection{Constant Volume Tap Density (CVTD) Method}

In contrast to the standard method of tap density measurement (CWTD) in which the mass of the test sample is fixed (100g in the case of gold powder), the CVTD method used a volume of the test sample which is fixed. In this method three cylinders with different heightto-diameter aspect ratio (as shown in Table 6-1) were used to measure tap density as also used for apparent density measurements. Powder was poured through the Scott volumeter into the cylinders. Excess powder was scraped with a glass slab and weighed. Extension pieces were placed and powder was added again from the Scott volumeter. The cups were tapped and after removing the extensions the excess powder was scraped and the cup was weighed to give the tap density after specific number of taps. Figure 6-11, 6-12 and 6-13 shows tap density measurement for Cup \# 1, Cup \# 2 and Cup \# 3 by the CVTD method respectively. Figure 614 shows the CWTD of standard $25 \mathrm{~cm}^{3}$ graduated glass cylinder and CVTD of Cup \# 1, Cup \# 
2 and Cup \# 3. It is noted that there is very little difference between the tap densities measured by CWTD and CVTD methods. The average difference between the tap densities obtained by CWTD and CVTD method was noted to be approximately $0.3 \mathrm{~g} / \mathrm{cm}^{3}$, which is a difference of approximately $3 \%$. Also a small variation in the dimensions of the density cups has a negligible effect on the tap density of the gold powder. The difference between the tap densities measured with these density cups was approximately $0.4 \mathrm{~g} / \mathrm{cm}^{3}$, which is a difference of approximately 4\%. Both in CWTD and CVTD methods, there is a jump between the first and second reading which indicates that gold powder compacted very quickly initially while after 60 taps the density remained almost constant.

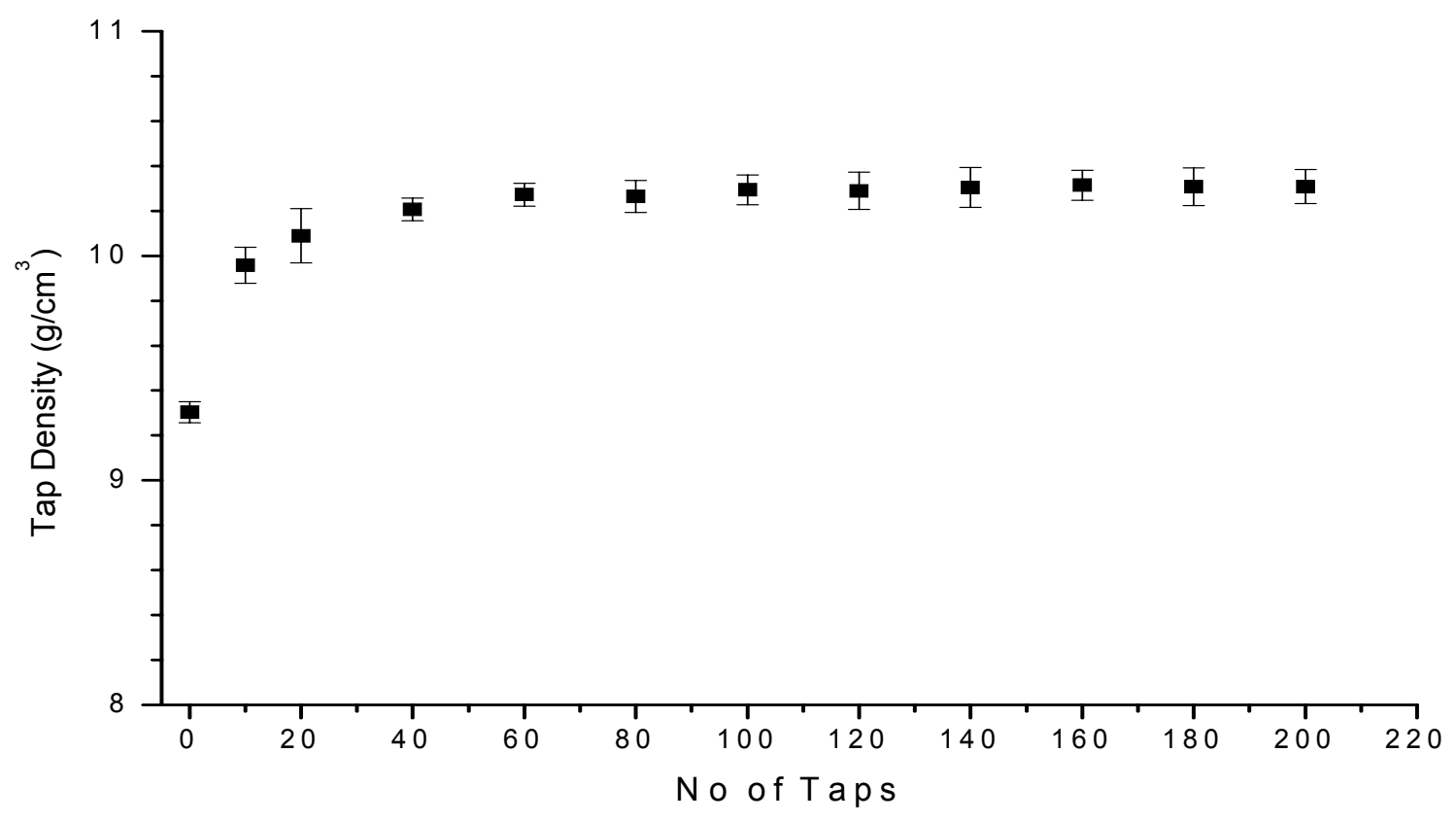

Figure 6-11: CVTD of gold powder with cup \# 1 


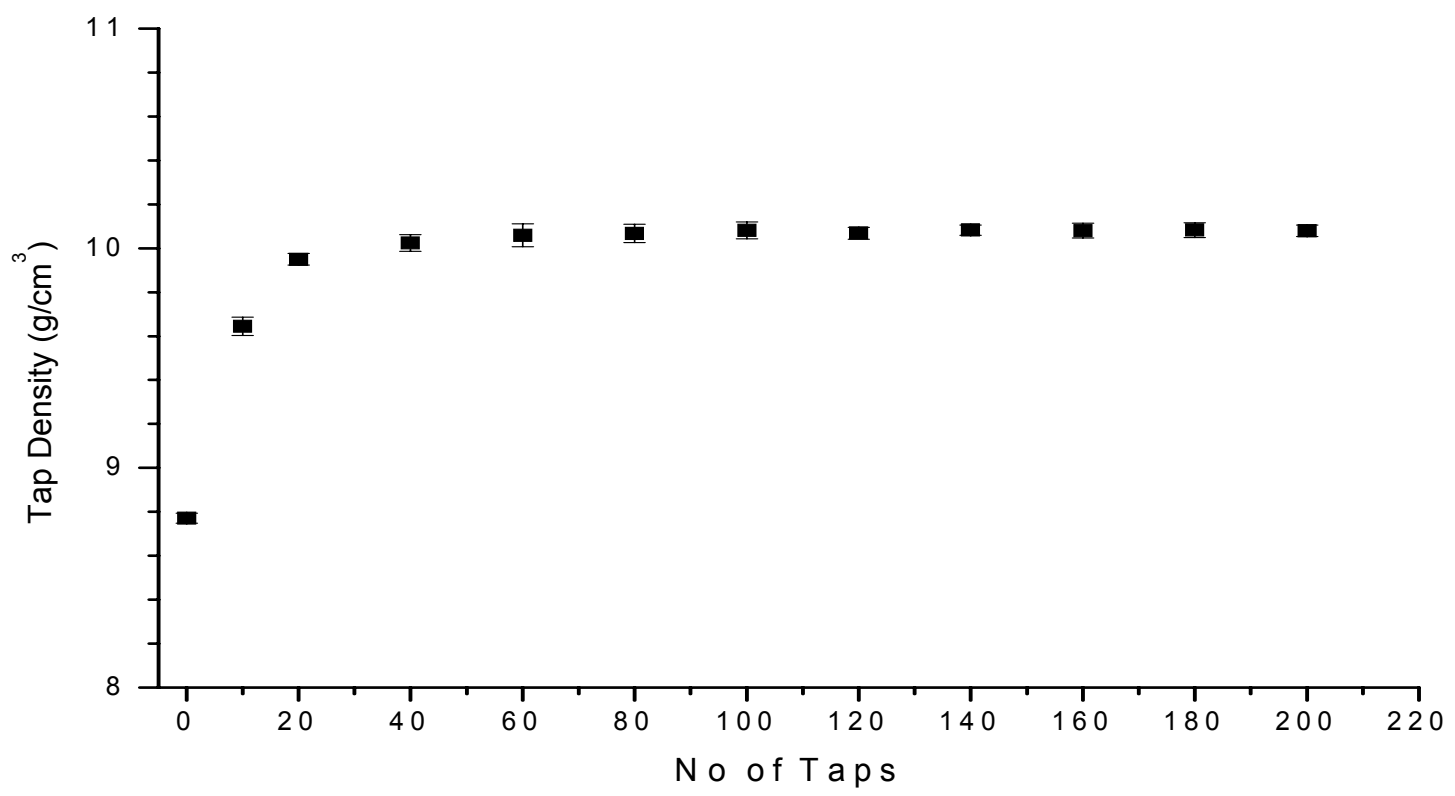

Figure 6-12: CVTD of gold powder with cup \# 2

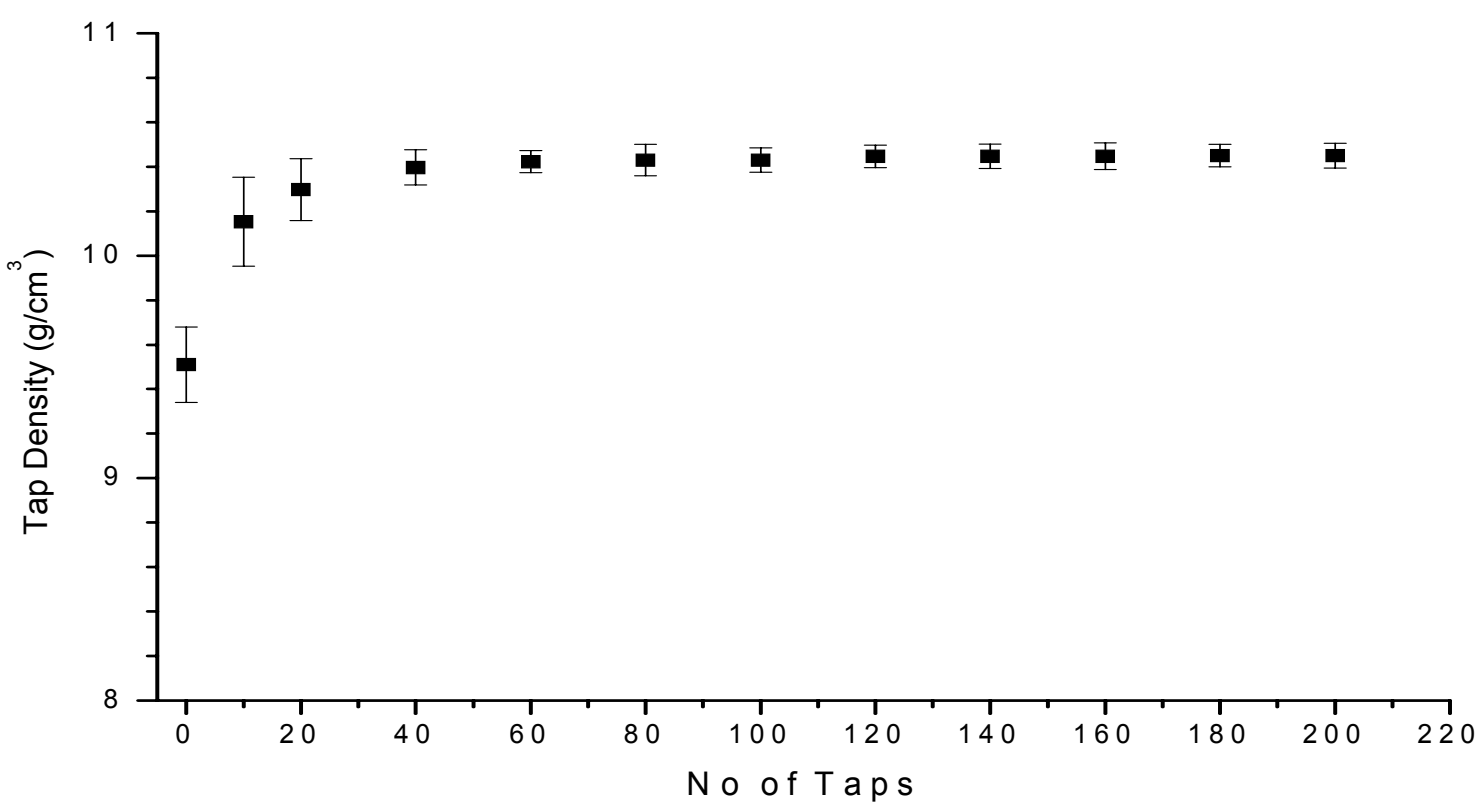

Figure 6-13: CVTD of gold powder with cup \# 3 


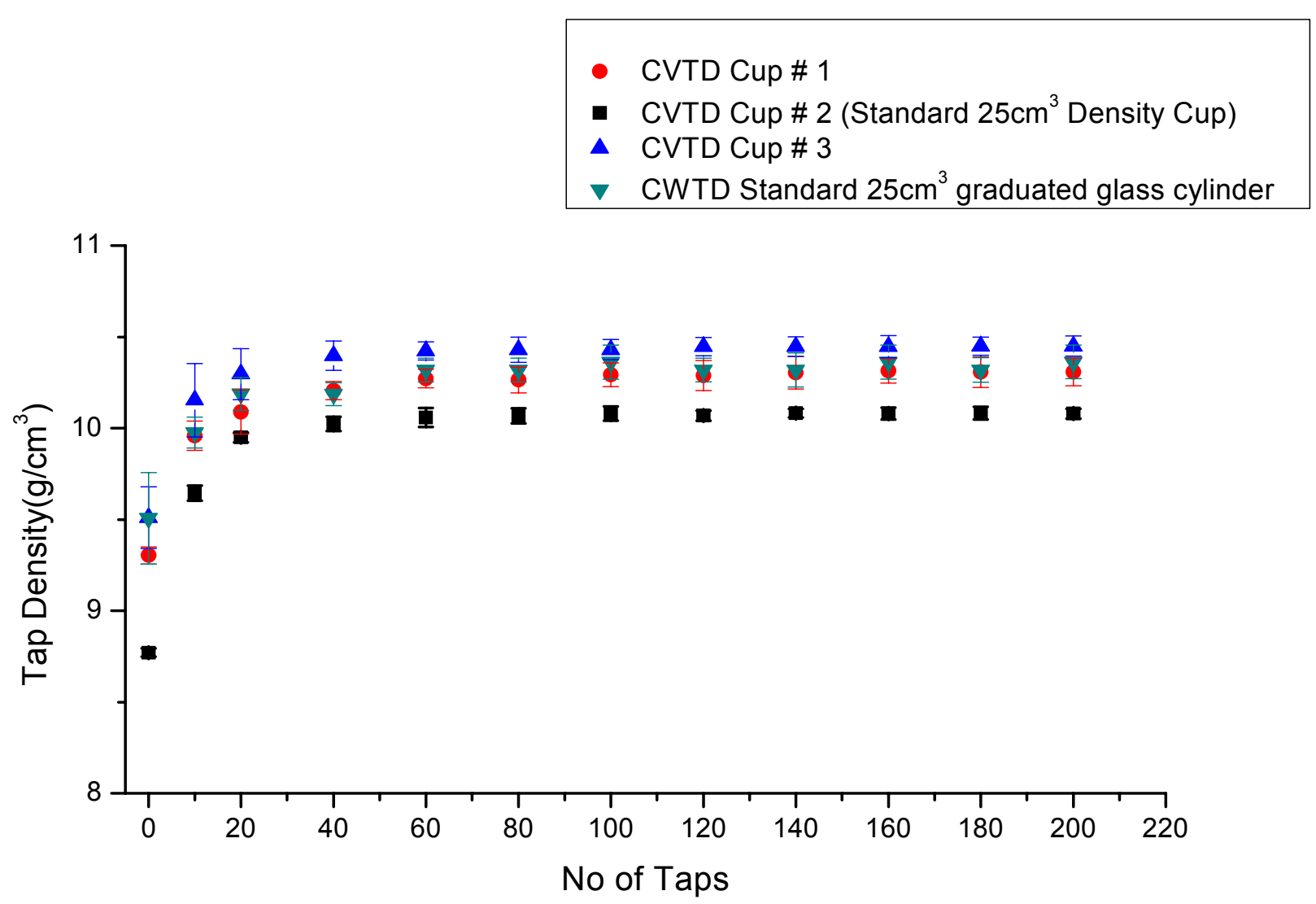

Figure 6-14: CVTD and CWTD for different density cups

\subsubsection{Hausner Ratio (HR) and Compressibility of Gold (Au) Powder}

The Hausner ratio is used as the Flowability indicator of powders. HR is calculated as the ratio of tap density to the apparent density (Scott density). The Hausner ratio was first introduced as a characteristic of the friction condition in the metal powders (Hausner, 1967). However, it has been used in measuring flow characteristics, sieving and compaction of metal powders (Svarovsky, 1987). The Hausner ratio has also been used to characterize the tapping behaviour of powders (Yu and Hall, 1994). Geldart \& Wong (1984) observed the HR to be independent of moisture content or environmental humidity, whereas Harnby et al (1987) found the contrary. Table 6-2 shows apparent density, tap density, Hausner ratio and compressibility of gold powder. Some researchers (Harnby et al, 1987; Abdullah \& Geldart, 1999) used HR as the ratio between tap and aerated density, while others (Wong, 2000) used tap and poured densities. Zou andYu (1995) used the apparent density determined by the 
method of Scott (1960). Here, as gold powder was not-freely flowing through a 2.5 or $5 \mathrm{~mm}$ orifice of the Hall flowmeter funnel or Carney funnel, so a Scott volumeter was used to measure apparent density. Figure 6-15 shows HR for different aspect ratio density cups including the standard $25 \mathrm{~cm}^{3}$ graduated glass cylinder.

\begin{tabular}{|c|c|c|c|}
\hline Density Cup & $\begin{array}{c}\text { Average Apparent } \\
\text { Density }\left(\mathrm{g} / \mathrm{cm}^{3}\right)\end{array}$ & $\begin{array}{c}\text { Average Tap } \\
\text { Density }\left(\mathrm{g} / \mathrm{cm}^{3}\right)\end{array}$ & $\begin{array}{c}\text { Compressibility } \\
(\%)\end{array}$ \\
\hline Cup \# 1 & 9.354 & 10.314 & 9.3 \\
\hline $\begin{array}{c}\text { Cup \# 2 (standard apparent } \\
\text { density cup) }\end{array}$ & 8.918 & 10.0827 & 11.55 \\
\hline Cup \# 3 & 9.511 & 10.45 & 7.99 \\
\hline $\begin{array}{c}\text { Standard 25 } \mathrm{cm}^{3} \text { Graduated } \\
\text { Glass Cylinder }\end{array}$ & 9.542 & 10.363 & 7.92 \\
\hline
\end{tabular}

Table 6-2: Apparent density, tap density and compressibility for different size density cups

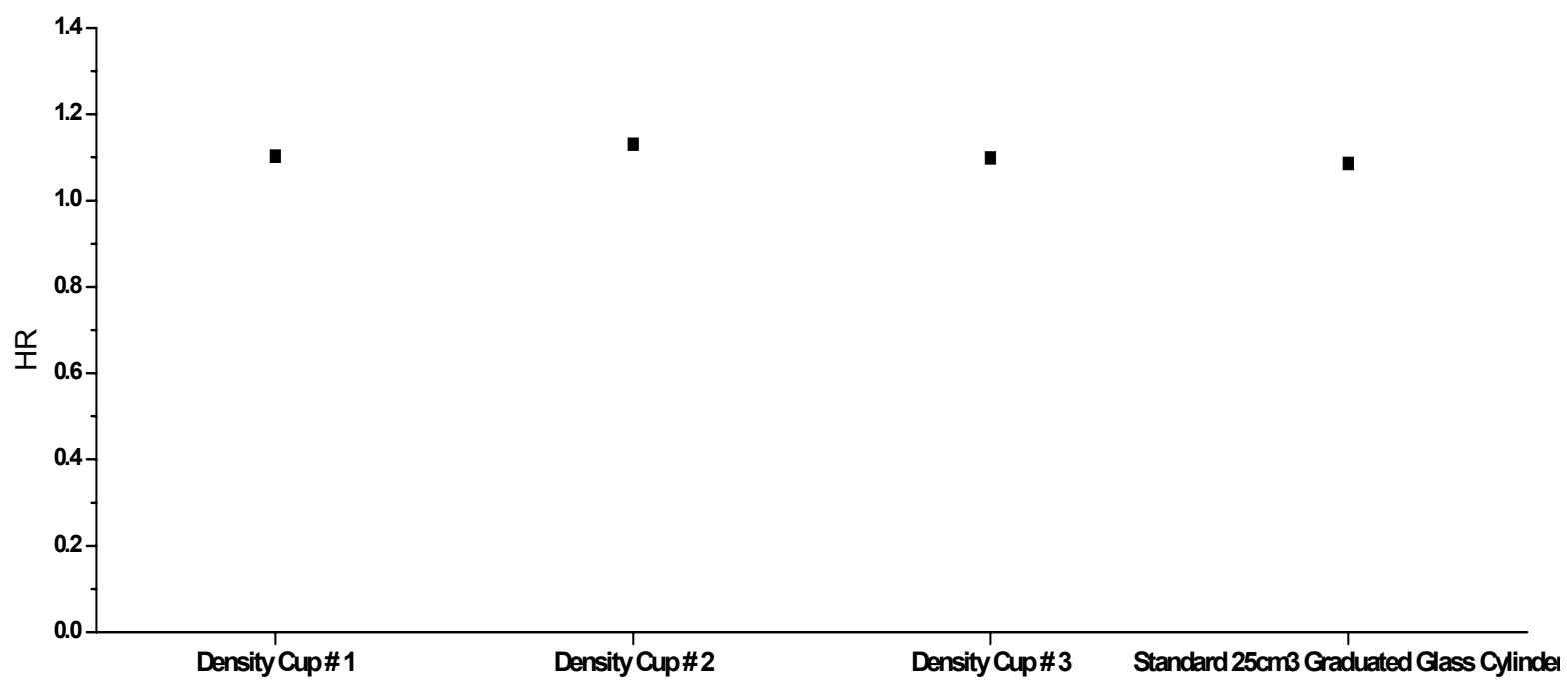

Figure 6-15: HR for different size density cups

Abdullah and Geldart (1999) considered a HR of 1.4 as the indicator for distinguishing cohesive and non-cohesive powders for Fluid Cracking Catalyst (FCC) and Fire Retardant Filler (FRF) but in our case, although gold powder was not flowing freely through the Hall flow meter funnel and Carney funnel (even with the external assistance of a metallic wire) the HR was below 1.4. The FCC and FRF used by Abdullah and Geldart (1999) had a particle density of $1.242 \mathrm{~g} / \mathrm{cm}^{3}$ and $2.449 \mathrm{~g} / \mathrm{cm}^{3}$ respectively, whereas the theoretical density of gold 
powder is $19.8 \mathrm{~g} / \mathrm{cm}^{3}$ which is quite high. Therefore, there is a need to check the effect of material density of powder particles on the apparent and tap density of the material. Abdullah and Geldart (1999) found that powders tested through the Hosokawa powder tester indicated a HR more accurately and sensitively than the Copley tap density volumeter (Figure 6-16), where the later showed a HR less than 1.4 for cohesive powders. Another factor expressing the apparent and tap density of powder is the compressibility. The compressibility of powder is defined by equation (6.3):

$$
\text { Compressibility }=\left[\left(\rho_{\mathrm{t}^{-}} \rho_{\mathrm{b}}\right) / \rho_{\mathrm{t}}\right] \times 100 \ldots \ldots \ldots(6.3)
$$

Where $\mathrm{p}_{\mathrm{t}}$ is the tap density, $\mathrm{p}_{\mathrm{b}}$ is the aerated or apparent density (Scott density in this case). Svarovsky (1987) characterized powders having compressibility greater than $20 \%$ to create bridges in hoppers and that of greater than $40 \%$ to be very hard to discharge, whereas compressibility of gold powder was found to be well below $20 \%$. The gold powder in these experiments created bridges and was difficult to discharge from the Hall and Carney funnels.

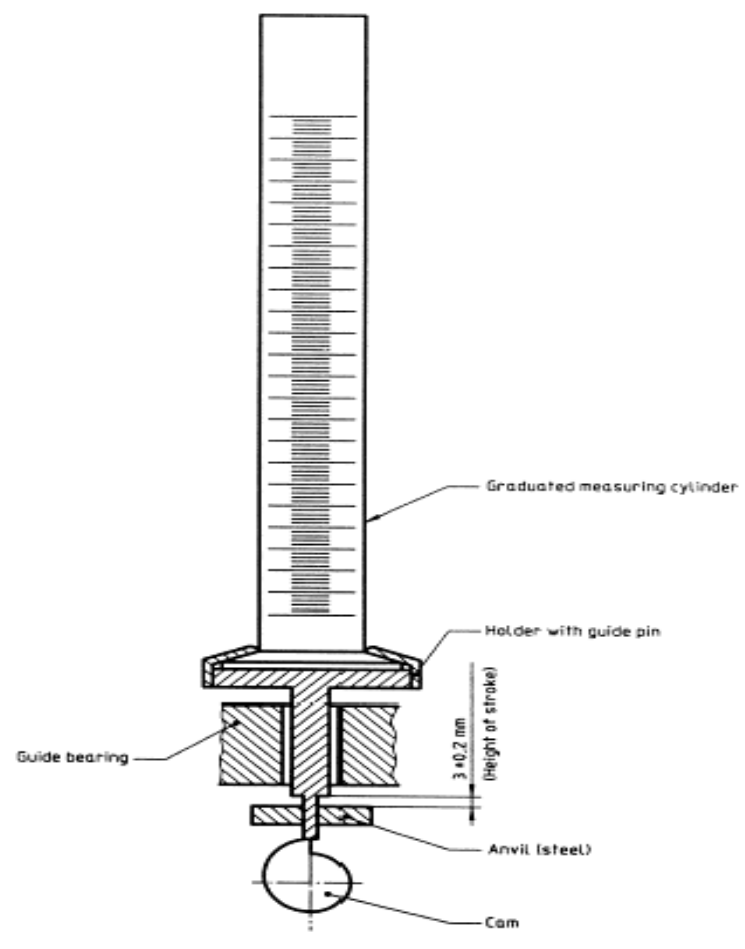

Figure 6-16: Copley tap density volumeter

\subsubsection{Powder Moisture Test}

The gold powder was tested for its moisture content. The powder moisture content is of particular importance to understand the powder flowability at different temperatures. In the 
previous experience of building parts with stainless steel powder, the powder frequently stopped flowing out of the hopper during the processing which resulted in scrap parts. By keeping the stainless steel powder at $150^{\circ} \mathrm{C}$ in the chamber for about an hour before the processing, the problem of flowability was successfully resolved. The main concern here was to check if gold powder had absorbed enough moisture from the air to make it less flowable during the density experiments and later in the processing.

A 50g sample of gold powder was used for measurement. The equipment used was a moisture analyzer from A\&D Company Ltd. The equipment measures the difference in weight of the samples at room temperature and at elevated temperatures. A high accuracy setting was used which took 1 minute to measure the weight difference. For gold powder, most of the moisture evaporates when the temperature is increased from room temperature to $50^{\circ} \mathrm{C}$. Stainless steel powder had higher moisture compared to gold powder. After the powders were heated to $100^{\circ} \mathrm{C}$ in the analyzer, the stainless steel powder was found to have had a moisture content of $0.028 \%$ compared to gold with $0.009 \%$. From these results it can be concluded that the moisture content of gold powder is not very high and the non-flowing nature of the gold powder cannot be altered to a greater extent by reducing moisture. Therefore, the cohesive nature of gold powder and the density results obtained in the previous sections are less influenced by the moisture of the powder. As powder bed pre-heating is required to eliminate the effect of thermal distortion in the part, this could also help in reducing the moisture content of the powder to be processed. The deposition process and powder bed pre-heating is discussed in detail in the following chapters on Chapter 7 and Chapter 8.

\subsection{Summary}

Different powder properties like particle size distribution, apparent density and tap density of gold powder have been presented. Particle size distribution was performed in water and isopropanol as a dispersant. The results obtained from these two were quite different, where maximum particle size was almost double in water as compare to isopropanol. It was concluded that due to poor dispersion of gold powder in water (also evident from visual inspection) the average particle size was greater than that measured with isopropanol. This was also confirmed by the SEM images of the gold powder.

The apparent density of gold powder was performed using a Hall flowmeter funnel but because of the cohesive nature of the gold powder, it was difficult to flow through a 2.5 and a 
$5 \mathrm{~mm}$ orifice. Therefore a $25 \mathrm{~cm}^{3}$ brass density cup was used with a Scott volumeter to measure apparent density of the gold powder. Apparent density of gold powder obtained using a $25 \mathrm{~cm}^{3}$ cylinder was also compared with different height-to-diameter aspect ratio density cups. It was noted that there was very little variation between these readings. Similarly, tap density of gold powder was measured using standard (CWTD) and non-standard (CVTD) methods. Here as well, very little difference was found between the results obtained by standard and nonstandard methods.

As mentioned above, the HR value of 1.4 and compressibility of $20 \%$ for differentiating between cohesive and non-cohesive powder was not satisfied by the gold powder as was also noted by Abdullah and Geldart (1999) for the Copley tap density volumeter.

The next chapter will explain the equipment and setup used for SLM of gold powder with the required modifications to the powder spreading mechanism and build chamber. 


\section{Chapter 7}

\section{CHARACTERISTICS OF PROCESSING SYSTEM}

This chapter details the specifications of the SLM system and the setup used in this research for processing gold powder. The typical configuration of an SLM machine is explained following the modifications made in this existing setup for gold powder to optimize the material usage.

\subsection{System Setup}

The experimental setup consisted of a MTT Technologies Group's SLM 100 system. This section explains the laser specifications and the powder deposition mechanism for the SLM 100 machine.

\subsubsection{Laser Assembly}

The SLM 100 system uses a 50 watts continuous wave ytterbium doped infrared fibre laser at a wavelength of $1070 \mathrm{~nm}$ to $1090 \mathrm{~nm}$. The SLM 100's scanning system incorporates an f-theta lens and uses the same layer based techniques as the majority of other Solid Freeform Fabrication (SFF) processes. In the SLM 100 machine, a wiper spreads a single layer of powder on the surface of a $125 \mathrm{~mm}$ diameter steel substrate. This is followed by the fiber laser scanning a single cross-sectional layer of the part. This process is repeated multiple times to create 3-dimesional metallic parts. Having a very small laser spot size and smaller build area, the SLM 100 is highly suitable for processing precious metals and alloys. The SLM 100 is also ideally suited for highly detailed and customized small parts such as jewellery items and dental crowns, cups and implants etc. Figure 7-1 shows the schematics of the SLM 100 setup and Figure 7-2 shows the SLM 100 chamber indicating lens, light, hopper, wiper and the build platform. Complete specifications of the laser and chamber are detailed in Table 7-1. 


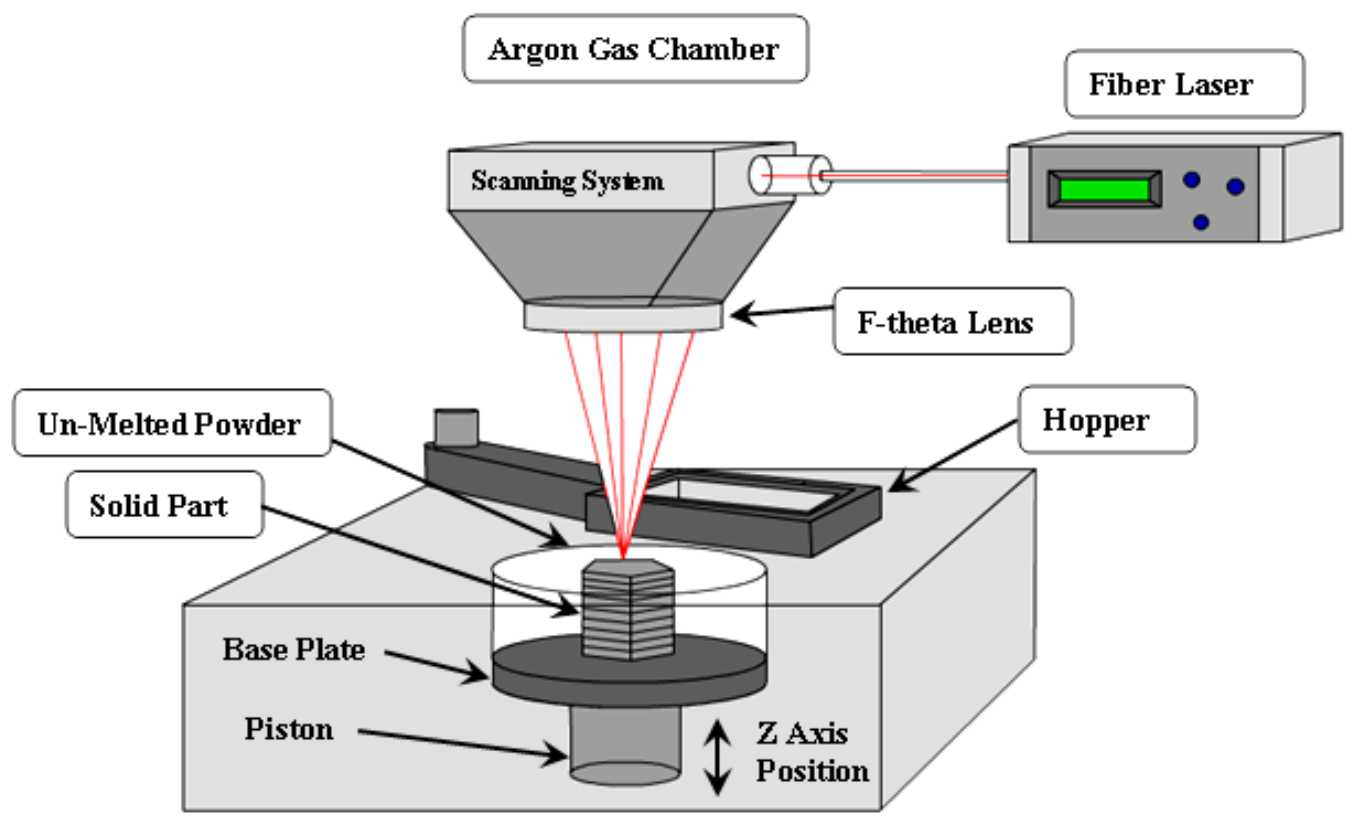

Figure 7-1: Schematics of the SLM 100 machine

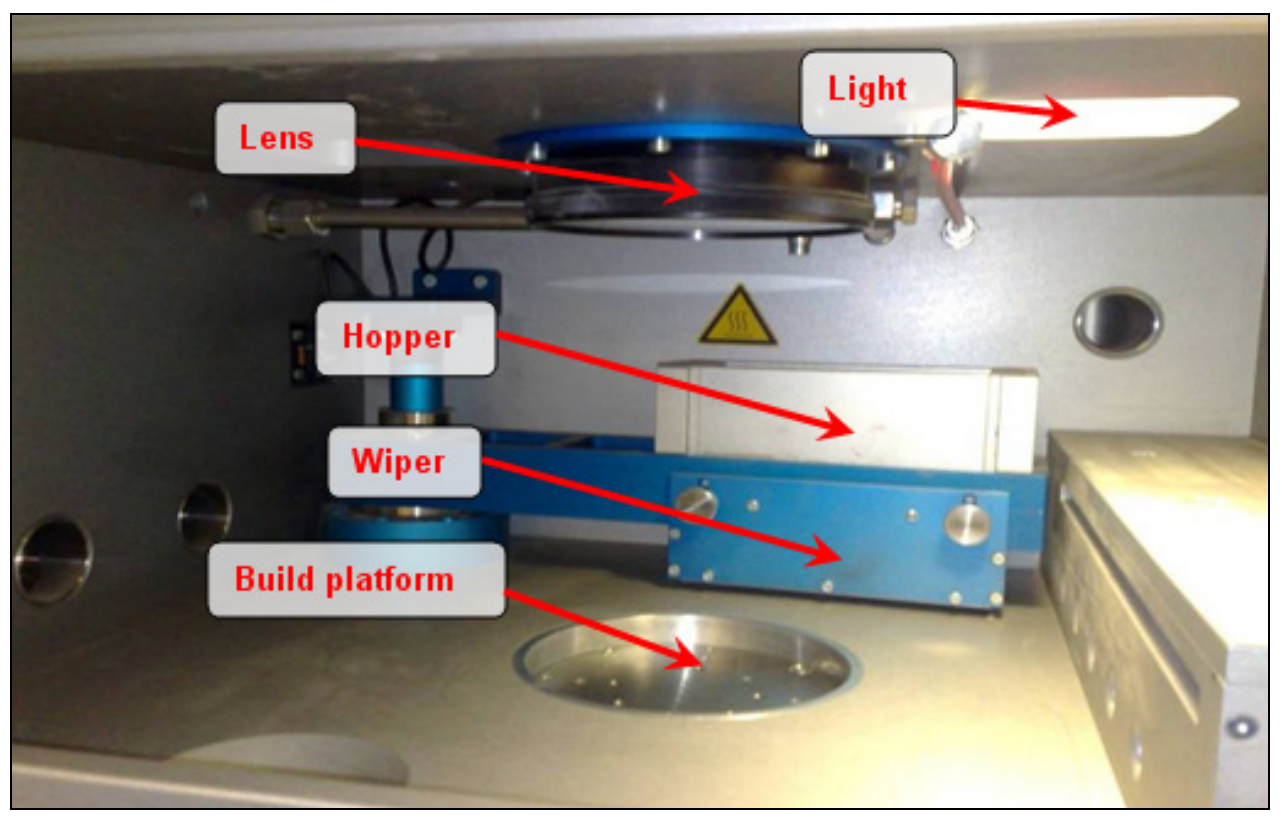

Figure 7-2: SLM 100 chamber 


\begin{tabular}{|c|c|c|c|}
\hline \multirow{12}{*}{$\frac{a}{x}$} & Parameters & Unit & YLM - 50 \\
\hline & Mode of operation & & Continuous wave \\
\hline & Central emission wavelength & $\mathrm{nm}$ & $1070-1090$ \\
\hline & Nominal output power ${ }^{1}$ & W & 50 \\
\hline & Output power stability (long-term) & $\%$ & $+/-2$ \\
\hline & Wavelength & $\mathrm{nm}$ & 1070 \\
\hline & Typical beam quality & $M^{2}$ & $<1.1$ \\
\hline & Output fibre delivery & & Single Mode \\
\hline & Maximum modulation speed & $\mathrm{kHz}$ & 40 \\
\hline & Electrical requirements & $\mathrm{VAC}$ & $110 / 240$ \\
\hline & Maximum power consumption & W & 240 \\
\hline & Cooling method & & air \\
\hline \multirow{3}{*}{ 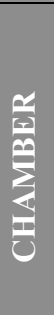 } & Powder deposition system & & $\begin{array}{c}\text { Cantilever rotating } \\
\text { wiper }\end{array}$ \\
\hline & Preheating temperature maximum & $\left({ }^{\circ} \mathrm{C}\right)$ & 250 \\
\hline & Oxygen level control & $(\%)$ & Less than $1 \%$ \\
\hline
\end{tabular}

\subsubsection{Powder Deposition System}

Powder deposition in the SLM 100 machine is performed by a wiper. The wiper is a cantilever beam with a housing at the end which holds the hopper. The hopper contains powder and it is specifically designed to spread powder evenly on the build area through multiple $2 \mathrm{~mm}$ diameter holes. In order for the powder to flow through these holes, the powder must be non-cohesive and free flowing. As gold powder was cohesive and non-free flowing (explained in detail in Chapter 6), it was thus necessary to design a new hopper which is explained in detail in section 7.6. The existing wiper in the SLM 100 machine can rotate through $110^{\circ}$ (as shown in Figure 7-3) to spread powder on the substrate. 


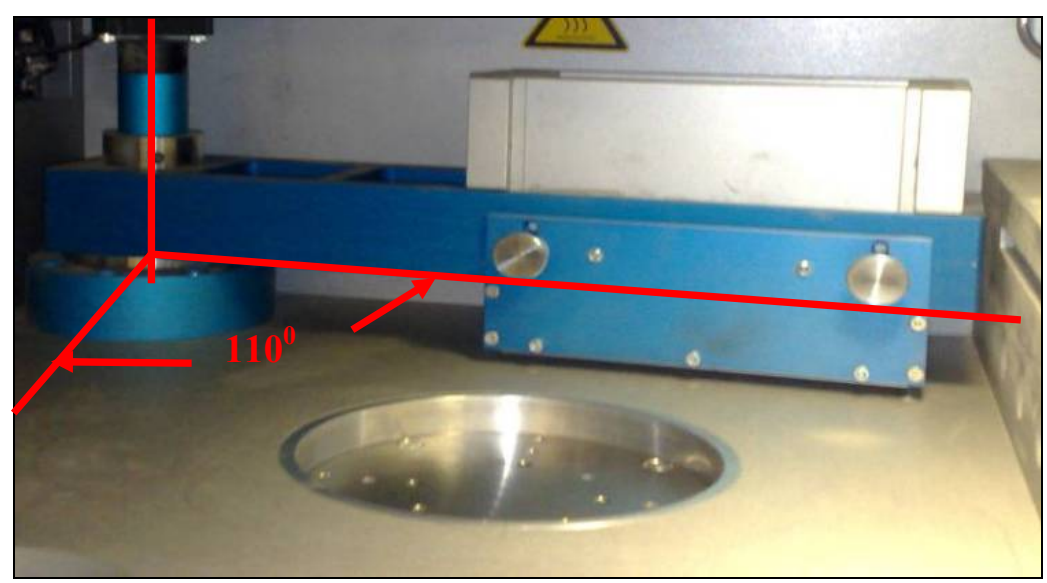

Figure 7-3: Wiper rotation in the SLM 100 machine

\subsection{Process parameters}

The SLM process parameters can be divided into four main groups i.e. material, laser, scan and environmental parameters. This section gives a brief description of these parameters.

\subsubsection{Material Parameters}

Once a material is selected for processing, some of the material properties are fixed. These properties include material density, heat capacity, latent heat of fusion and melting temperature. These properties define the melt enthalpy which is the actual quantity of energy needed to melt the material. Therefore, after the material selection, its melt enthalpy can be determined. However, there are also other factors which also have considerable effect during the laser material processing. These properties include heat transfer through the powder bed and radiation absorption. Heat transfer and radiation absorption in powder bed depends on the powder bed compaction and phase of the material (solid or liquid) which have already been discussed in Chapter 5 and Chapter 6.

\subsubsection{Laser Parameters}

Since the SLM uses thermal energy of a laser beam to melt the powder, so properties of a laser are extremely important. Several other important parameters like wave length of the laser, mode, beam quality and spot size etc are fixed for the system. Unlike pulse laser systems, only the laser power could be varied for the continuous wave SLM 100 system. 


\subsubsection{Scan Parameters}

The scan parameters determine the way a slice is scanned by the laser beam and it has a great influence on the build rate, surface roughness and quality of the SLM part. The scan parameters are the scan speed, the hatch spacing, percentage overlap and scan strategy (single-scan or cross-scan etc).

\subsubsection{Scan Speed}

Scan speed is the speed with which laser scans the cross-section of the part on the powder bed. The scan speed of a system directly influences its productivity.

\subsubsection{Hatch Spacing}

Hatch spacing is the distance between two successive scan tracks. A certain amount of overlap is necessary between two consecutive hatch lines to avoid porosity and provide better layer integrity. Figure 7-4 shows the hatch distance between two consecutive beads.

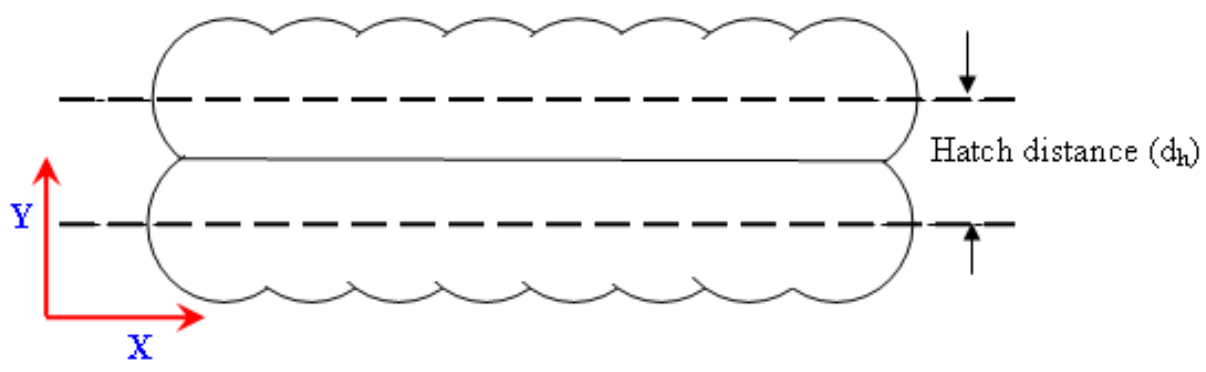

Figure 7-4: Hatch distance for two consecutive beads

\subsubsection{Scan Strategy}

The scan strategy is the method used to fill a single cross-section with the scan lines. The cross-section can be subdivided into smaller sectors that can be scanned in a certain order following a certain pattern (zig-zag, spiral, hatching, etc.). Different scan strategies can also be used to reduce stresses in the parts (Kruth et al, 2004)

\subsubsection{Environmental Parameters}

Controlling the atmosphere during SLM processing such as oxygen level and pressure is of vital importance as the presence of oxygen leads to oxidation of the metal. Powder bed temperature regulation is also very important because high powder bed temperature reduces the thermal gradient between melted and un-melted powder hence reducing thermal stresses. In this research gold powder was used which does not oxidize even in normal atmospheric conditions. However, due to the strict safety control of the 
SLM 100 system, it was not possible to perform experiments without filling the chamber with argon gas.

\subsection{Laser Spot Size Measurements}

Laser spot size is usually measured by obtaining an image of the fiber output on the work piece. Due to the lack of equipment, a direct analysis of beam shape and energy distribution was not possible. A laser beam Analyzer (LBA) could not be used due to lack of space in the chamber and directing the laser beam outside the chamber was a serious hazard. Therefore, laser spot size measurement was first performed with kapton film at 50W. Due to the high power, it burned the paper and created very large sized spots. Therefore, lower laser power was used but no significant results were obtained with kapton film. Alternatively, black burn paper was used for spot size measurements. Figure 7-5 shows the template and black burn paper. The template was designed in such a way that it would keep the paper above the surface of the substrate to avoid overheating from the base plate underneath. This reduced the extra heat input to the paper and avoided over-burns. Figure 7-6 shows an image of the spot size measured under the microscope.
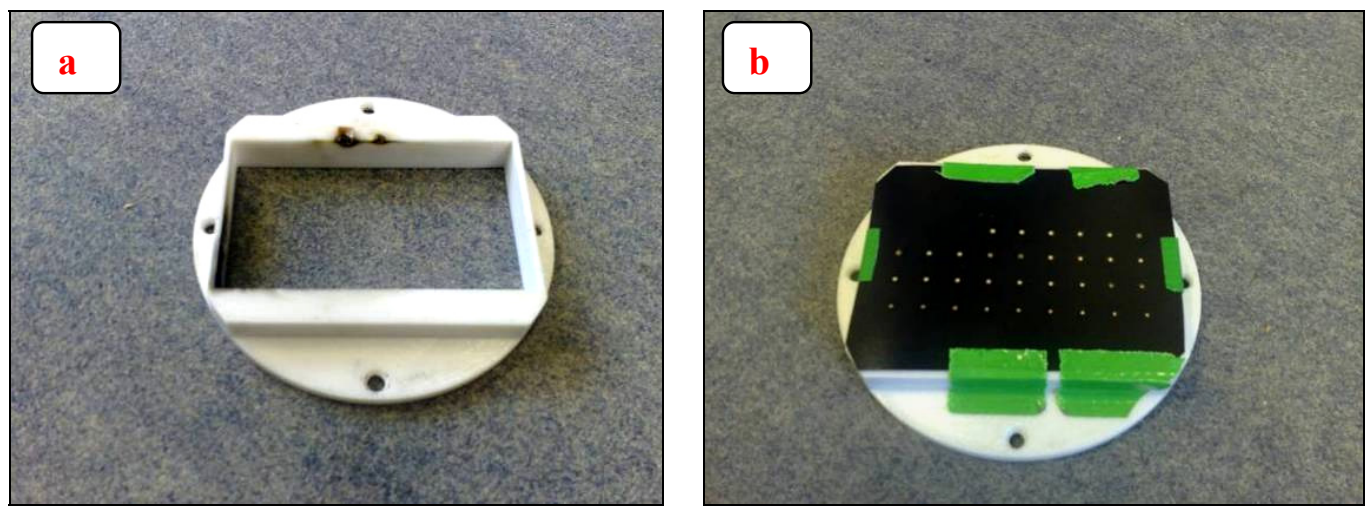

Figure 7-5: (a) Template and (b) Black burn paper taped to the template

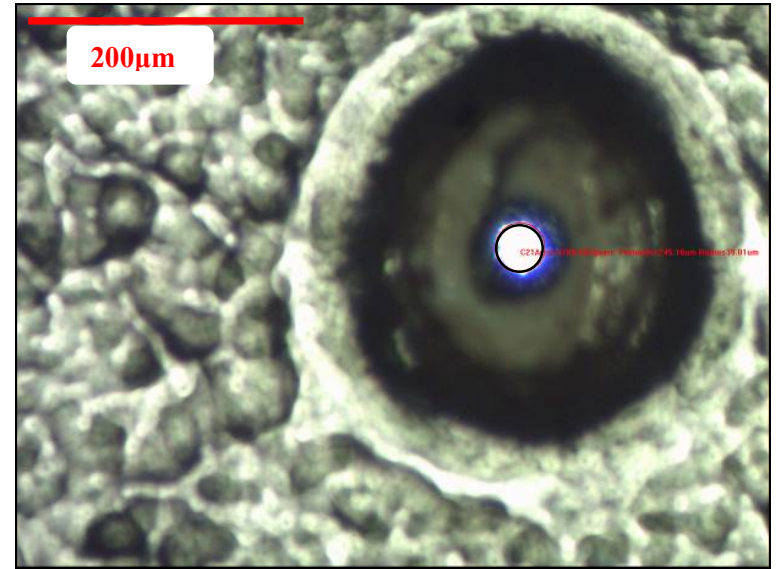

Figure 7-6: $\quad$ Spot size measurement $(35 \mu \mathrm{m})$ for YLM-50 laser on black burn paper 
The effect on the focused beam diameter (also known as laser focus) was determined by firing single shots of laser on the black burn paper with the incremental movement of the piston from $-5 \mathrm{~mm}$ to $+5 \mathrm{~mm}$, where ' 0 ' indicates the template position as level with the bed of the SLM 100 machine. If the movement was correct, the beam diameter should decrease to a minimum and then increase again. The distance where the beam diameter is a minimum is the laser beam waist diameter. The SLM 100 machine bed was then adjusted to be focused at this smallest beam spot. The beam diverges as it moves away from this point. This phenomenon is used as a technique in Laser Surface Remelting (LSR) to improve surface roughness and surface properties of the already sintered layer in RM parts (Gacsi et al, 2002). In LSR, the part is initially sintered with the optimized parameters to reduce its internal porosity and produce dense parts. At the same time, the outer surface is re-melted with a larger spot size and different laser power and scan speed settings, thus reducing the outer porosity and surface roughness.

A very low laser power of $3.9 \mathrm{~W}$ was found to be appropriate for spot size measurement as a higher power created over burns in the black burn paper and lower power could not produce a through burn. All the spots on the black burn paper were viewed under the microscope and measured by integrated camera software. This software was calibrated for the camera and the spot size was measured as shown in Figure 7-6. Figure 7-7 shows the spot size variation for the vertical position of the piston. The minimum spot size of $35 \mu \mathrm{m}$ was obtained for $3.9 \mathrm{~W}$ when the piston was level with the bed i.e. 0 level. It is evident that hole diameter in the black burn paper is proportional to the applied energy. As the applied energy increases, the hole size in the black burn paper also increases as shown in Figure 7-8. As the laser beam was focused at the SLM 100 bed level (as anticipated for a calibrated machine), there was no need to focus before every experiment.

The depth of focus also depends on the wavelength of the laser and f-number. The f-number is the ratio of focal length of the focusing optics to the incident beam diameter. Depth of focus provides a working range where the laser energy density will not decrease more than $10 \%$ due to the increase of the diameter. Therefore it gives a tolerance to position the base plate anywhere in this region while still maintaining the almost the same energy input as at the focus which in turn enhances the repeatability of the results (Daves, 1991). 


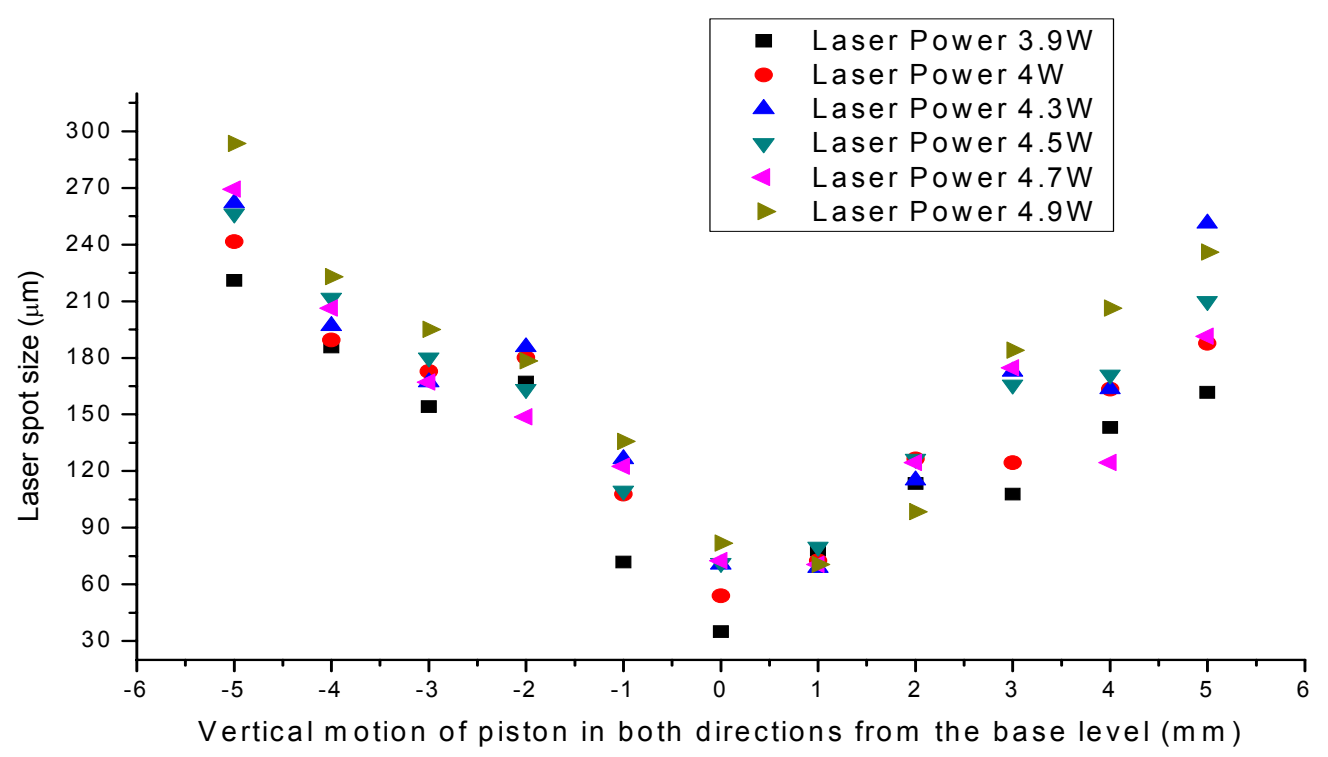

Figure 7-7: Laser spot size vs position of the piston

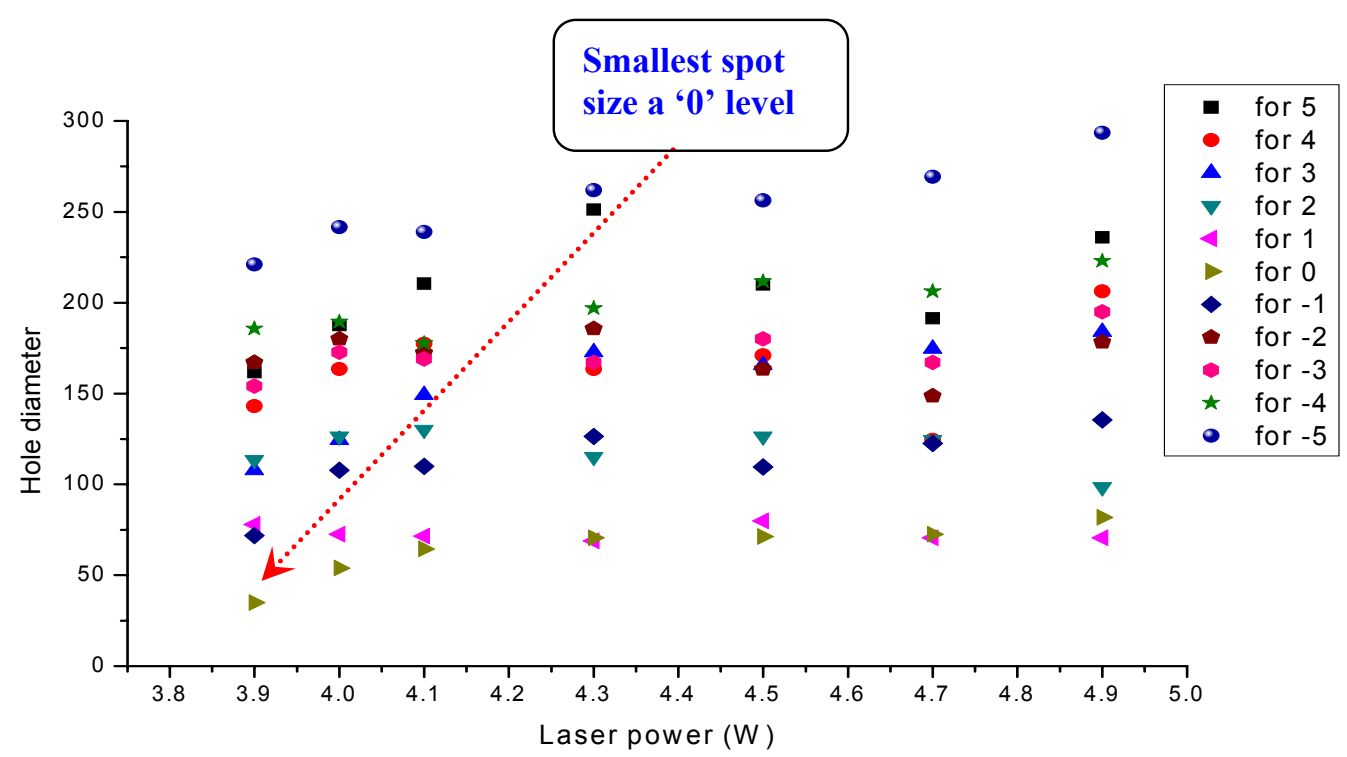

Figure 7-8: Laser spot size variation with laser power for different piston levels from -

$$
5 \mathrm{~mm} \text { to }+5 \mathrm{~mm}
$$

\subsection{Laser Power Measurements}

Laser optical power output was obtained by a laser power meter placed on the bed of the SLM 100 system while laser beam was fired at the specific points.

The SLM 100 system takes input current value as the input power values from the software (for spot size measurements). For example, if an output power of $50 \mathrm{~W}$ was desired, laser power value of $50 \mathrm{~W}$ was input to the system, the machine considers this as $5 \mathrm{~A}$ input current and produces an output power of $49.6 \mathrm{~W}$ (as shown in Table 7-2). It can 
be seen in Table 7-2 that the output power was not equal to the input values which was due to the losses in the scanning system.

\begin{tabular}{|c|c|c|}
\hline $\begin{array}{l}\text { Input laser power value } \\
\text { (W) }\end{array}$ & $\begin{array}{l}\text { Equivalent laser input } \\
\text { current (A) }\end{array}$ & $\begin{array}{l}\text { Optical laser output } \\
\text { (W) }\end{array}$ \\
\hline 0 & 0 & No output power \\
\hline . & . & No output power \\
\hline & . & No output power \\
\hline 3.2 & 0.32 & 0 \\
\hline 3.3 & 0.33 & 0.3 \\
\hline 5 & 0.5 & 2.5 \\
\hline 10 & 1 & 9.3 \\
\hline 15 & 1.5 & 14.5 \\
\hline 20 & 2 & 19.8 \\
\hline 25 & 2.5 & 24.6 \\
\hline 30 & 3 & 29.4 \\
\hline 35 & 3.5 & 34.3 \\
\hline 40 & 4 & 39.7 \\
\hline 45 & 4.5 & 44.5 \\
\hline 50 & 5 & 49.6 \\
\hline
\end{tabular}

Table 7-2: Input Power, the resulting input current and the optical power output for the YLM-50 fiber laser

Figure 7-9 shows the laser output power for the input current. There is no output power for input current values of less than $0.32 \mathrm{~A}$. This is normal for the commercially available laser which requires a certain level of input current to give a minimum laser output. The difference between the input laser power value and the actual laser output was less than $1 \mathrm{~W}$ for the laser power above $10 \mathrm{~W}$. 


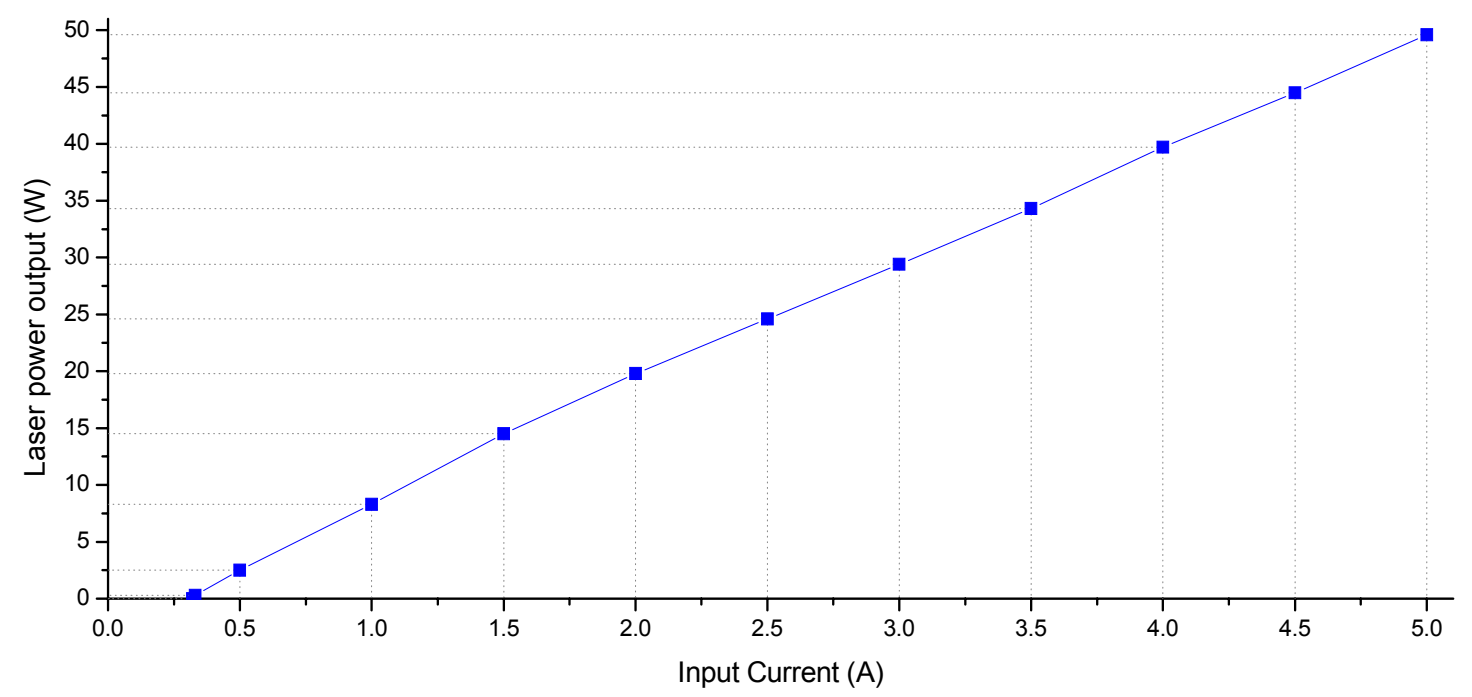

Figure 7-9: Input current and laser output power

\subsection{Modifications to the SLM 100 System}

This section explains in detail the modifications made to the SLM 100 powder delivery assembly, build platform and deposition system.

\subsubsection{Need for Modifications}

The SLM 100 system has a relatively smaller build volume $(\phi 125 \mathrm{~mm} \times 80 \mathrm{~mm})$ compared to other commercially available metal systems for example SLM 250 etc. As the gold powder available for this research work was very small in quantity (300g) and the dental parts to be manufactured were also relatively small, a much smaller build area was required. For this purpose a new platform with smaller build volume was designed.

The existing SLM 100 powder hopper was also too big for the limited amount of the material available, therefore a new powder hopper and deposition system was designed. The existing hopper had seven $2 \mathrm{~mm}$ diameter holes which allowed the powder to flow onto the substrate. This was then scraped off by the wiper blades. These small holes were good for freely flowing powders like H13 tool steel and stainless steel powders but were not allowing an even flow of gold powder. Keeping in view the non-flowing nature of the gold powder, the holes were replaced by a large opening in the new hopper ensuring efficient powder flow on to the powder bed.

Apart from these measures, wiper rotation was also reduced from 110 degrees for the smaller build volume hence optimizing the powder deposition process. A full detail of these modifications is explained in the next sections. 


\subsubsection{Powder Hopper Modifications}

Three different powder hoppers were designed specifically for gold powder deposition. These hoppers were much smaller in size than the existing SLM hopper. Out of these the best hopper was then selected based on the powder flowability, repeatability of deposited layers and the visual examination of layer thickness control.

Figure 7-10 shows the first hopper which has rectangular in shape. This hopper could be mounted using the two rectangular slots on each wing as shown in Figure 7-10a. The vertical and horizontal position of the hopper could also be adjusted for 50, 75 or $100 \mu \mathrm{m}$ layers. Powder was deposited through a $15 \mathrm{x} 20 \mathrm{~mm}$ rectangular cut at the bottom. Initially rectangular pads were used for the gold powder due to the cohesive nature of the powder and the scrapping action of the rectangular pads, smaller layer thicknesses (50 or $75 \mu \mathrm{m})$ could not be achieved. The rectangular pads also damaged the previous layer on certain occasions. The rectangular pads were then replaced by a red circular silicon cord (from Silex Ltd) which was placed on each side to scrape the gold powder into the desired layer thickness. The circular cord was preferred over the rectangular shape pads because of the compaction action. The rectangular hopper was installed inside the machine and it did produce some good layers but its repeatability was poor. Apart from this, its installation especially its horizontal levelling by feeler gauges was very difficult and setting up time was very long.
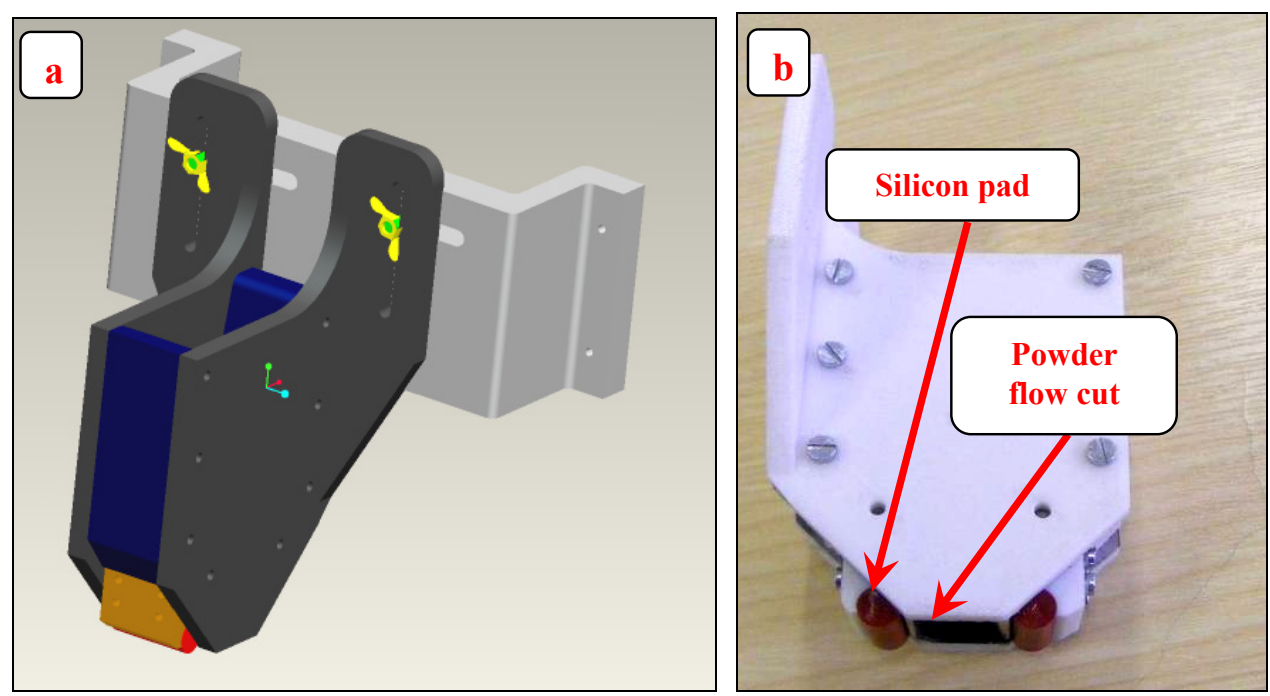

Figure 7-10: Rectangular hopper (a) CAD model with complete installation assembly (b) FDM model 


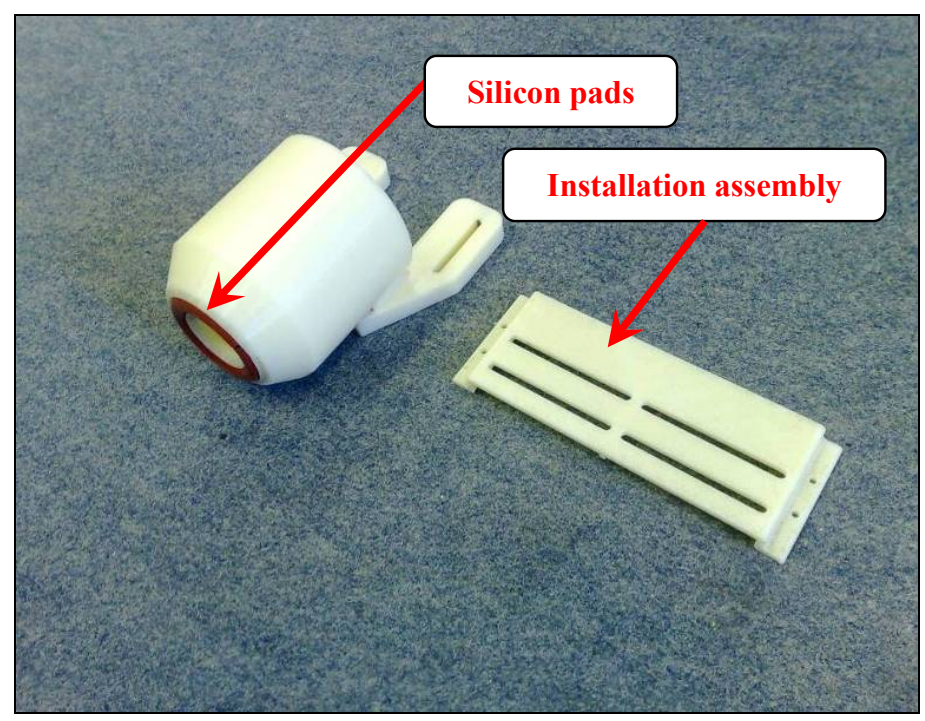

Figure 7-11: Circular hopper and the installation assembly

The second hopper designed for deposition was circular in shape. Unlike the rectangular hopper, where two silicon cord pads were used to level the powder, the circular hopper had a circular ring of silicon cord to level the powder. Figure 7-11 shows the circular hopper with a rectangular slot attachment which was used to mount and level the hopper. The circular hopper had a $20 \mathrm{~mm}$ diameter hole for powder to flow through. The circular hopper showed good results with powder flowability and smoothness of the deposited layers but had problems with horizontal alignment during its installation and therefore its repeatability was very poor.

In both the rectangular and circular hoppers, their attachment to the outer side of the lever arm (SLM 100 wiper) made it very difficult to hold them in the horizontal position during operation. This was found to be the reason for their low repeatability. Keeping these things in mind, a new hopper was designed (shown in Figure 7-12) which could fit into the existing SLM 100 setup, it was smaller in size than the original hopper (Figure 7-13) and easily assembled and fitted onto the machine. Figure 7-12c shows the selected hopper installed in the SLM 100 chamber. The layer deposition was good as in the case of previous hoppers but due to its good horizontal alignment, its repeatability was very much improved. The horizontal alignment was done with feeler gauges. Due to its easy instalment, the setup time was reduced as well. Section 7.5.8 shows the layers deposited with this selected deposition system. 

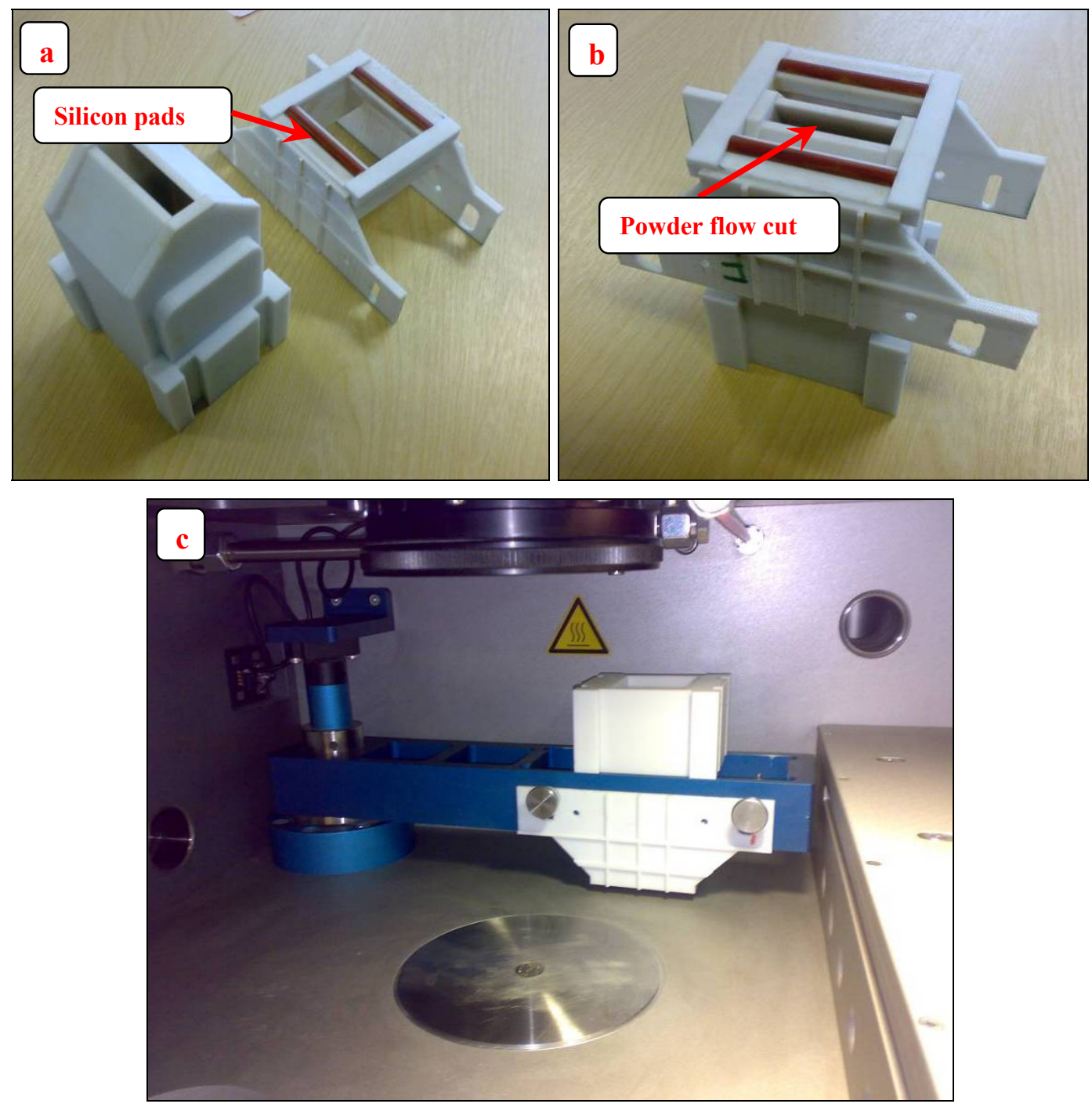

Figure 7-12: Selected smaller hopper (a) disassembled (b) assembled (c) hopper assembled and installed inside the SLM 100 chamber

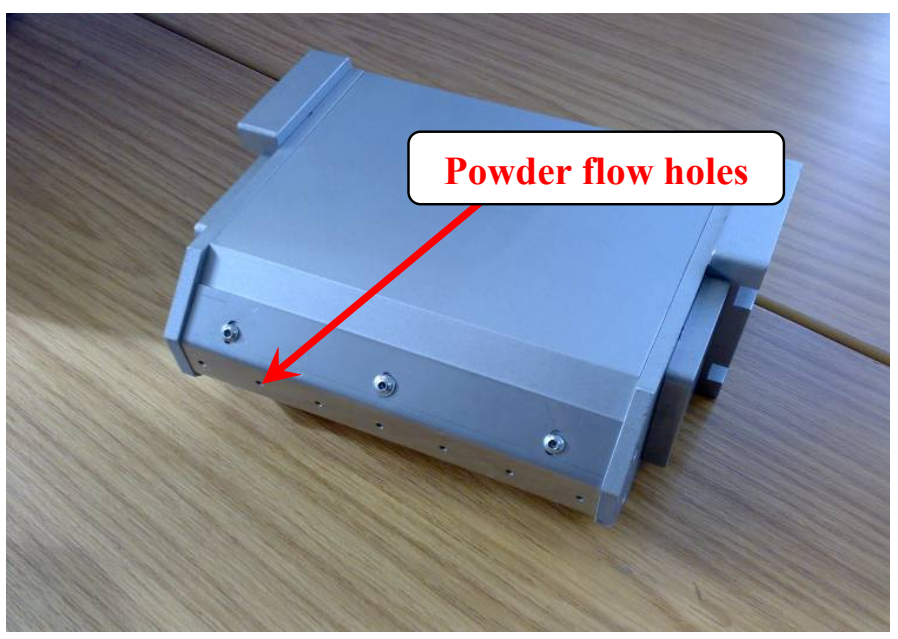

Figure 7-13: Original SLM 100 hopper 


\subsubsection{Build Platform Modifications}

There was a need to reduce the build volume to build small dental parts. This facilitated producing test samples even with a small amount of material. The new piston and bed were made from stainless steel, with total build volume of $\phi 15 \mathrm{~mm} \mathrm{X} 20 \mathrm{~mm}$ depth in the Z-direction. Figure 7-14 shows the new piston and build platform.
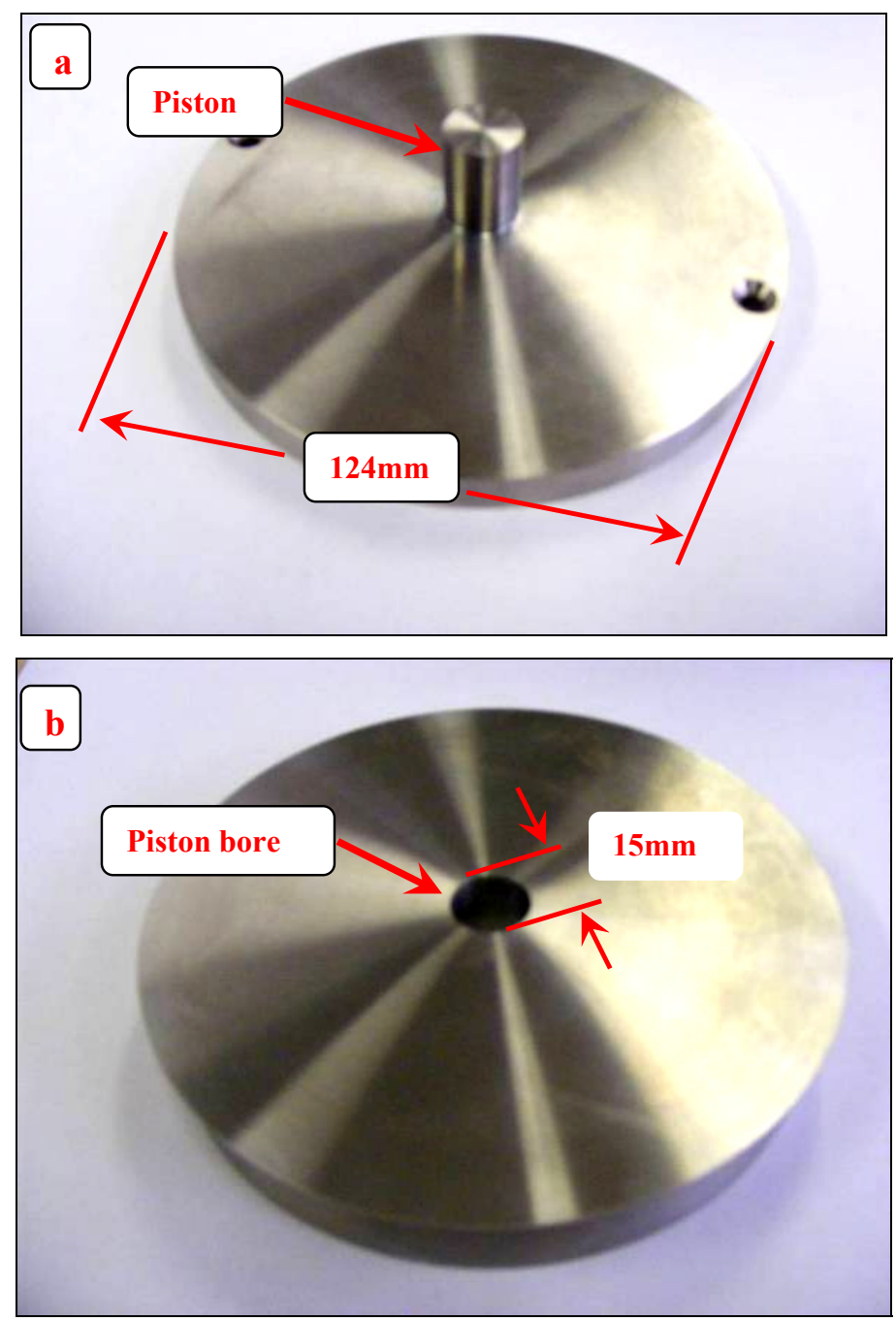

Figure 7-14: New platform (a) smaller piston and (b) build platform with bore

The new platform was capable of moving with the same resolution and accuracy in Z-direction (layer thickness) as the original SLM 100 system. The new platform was mounted onto the piston and checked repeatedly for any powder sticking in between the piston and the bore. This could result in damaging the bore and piston, which would in turn affect the layer accuracy. No such effect was seen for the new piston and platform. This new platform was easily operated from the existing SLM 100 panel and could produce 
parts with the same layer thickness as the SLM 100 system. Figure 7-15 shows the schematic of the new piston and platform mounted in the machine chamber. In each step, the wiper would spread a single layer of powder onto the surface of the small bore, which would be lowered one layer thickness each time, followed by the laser scanning the cross section. The same process would be repeated multiple times to create $3 \mathrm{D}$ objects.

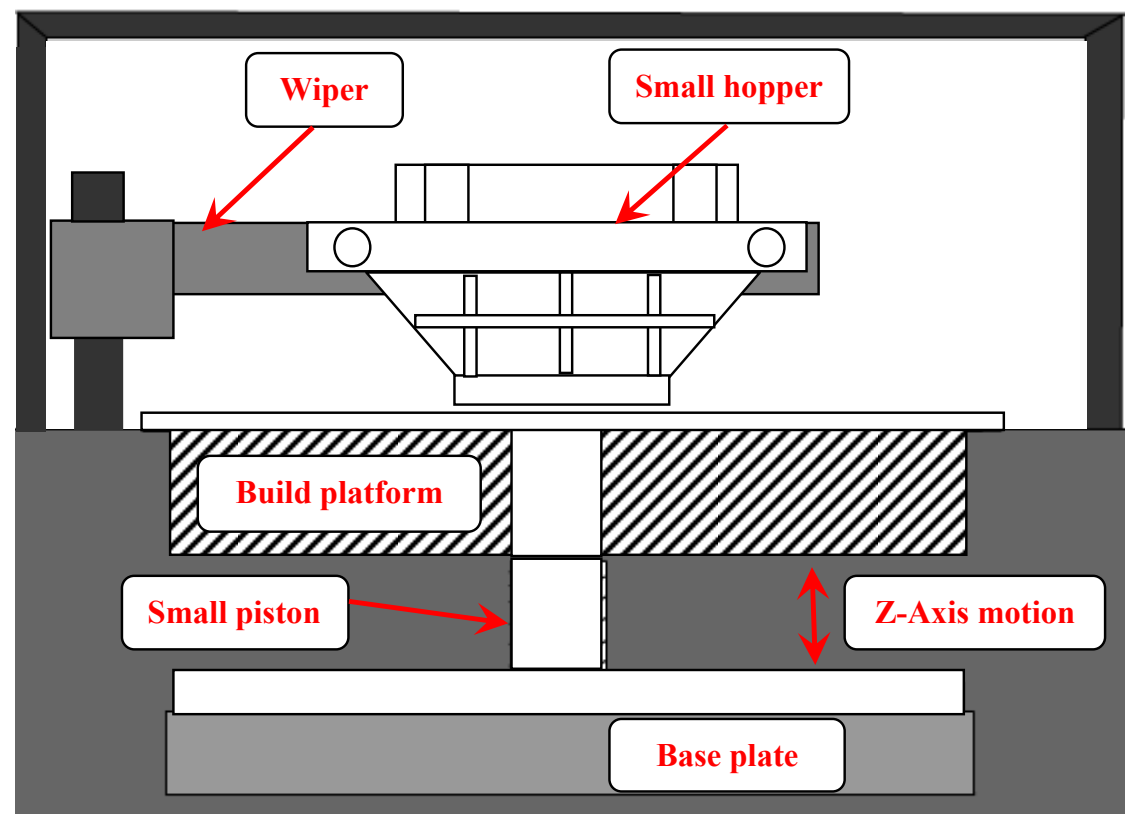

Figure 7-15: Arrangement of the new piston, base and hopper for the reduced build platform

\subsubsection{Prevention of Contamination of Gold Powder}

As the SLM 100 system was also being used for other metal powders (stainless steel etc), it was critical to keep the gold powder free of contamination. Although, the machine was thoroughly cleaned before using it for gold powder in order to eliminate any chance of contamination a new chamber floor was designed. This chamber floor was made of a transparent plastic sheet (Figure 7-16) with appropriate cutouts for the build platform. This new chamber floor could be fixed directly into the existing chamber with ease and did not restrict the movement of the new hopper and deposition system. The plastic sheet was capable of maintaining its shape up to $200^{\circ} \mathrm{C}$, which was needed for high bed temperatures. This new plastic floor also helped in the collection of powder after processing. 


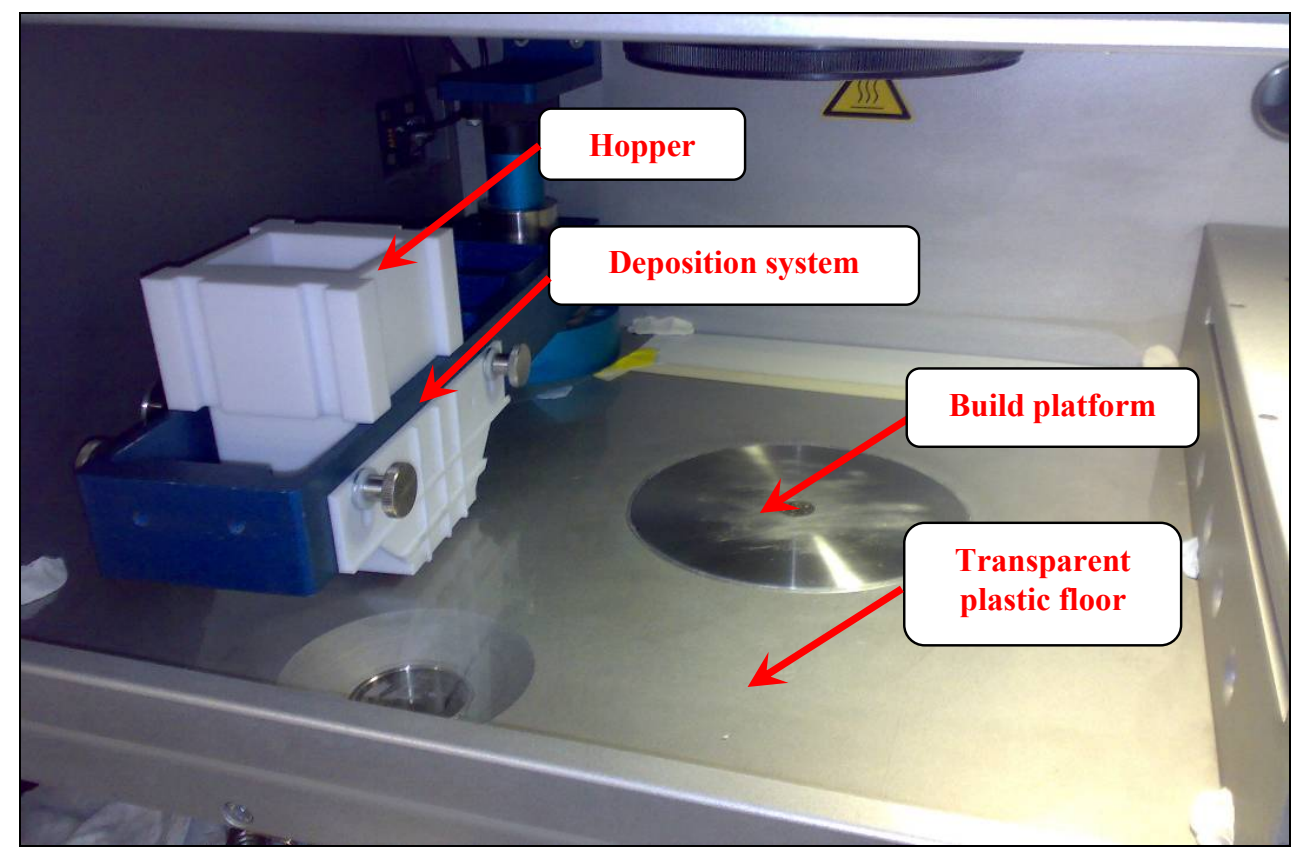

Figure 7-16: Plastic floor in the chamber with new deposition system and smaller build platform

\subsubsection{Layer Thickness Control for the New Setup}

To control the layer thickness deposited by the new hopper, it was first screwed to the supporting guides while height from the bed was adjusted by feeler gauges. A $100 \mu \mathrm{m}$ gap between the silicon pads and the bed was found to be suitable for depositing smooth layers. The feeler gauge adjustment was done every time the assembly was installed in the SLM 100 system.

\subsubsection{Wiper Rotation Control for the New Setup}

In the SLM 100 system, the wiper is rotated through $110^{\circ}$, but for the smaller build platform and hopper, the wiper rotation was limited to $15^{0}$ i.e. from $10^{0}$ to $25^{0}$. This not only helped in speeding up the layer deposition process but also reduced the spreading of powder inside the chamber, making it easier to collect and minimize its wastage.

\subsubsection{Bed Temperature of the New Build Platform}

The SLM 100 is capable of reaching a bed temperature up to $250^{\circ} \mathrm{C}$. However, for the new build platform, at temperatures higher than $100^{\circ} \mathrm{C}$, the smaller piston stuck in the bore due to thermal expansion and the process was stopped. This limited the maximum bed temperature with the new platform to $100^{\circ} \mathrm{C}$. A higher bed temperature is known to reduce the energy required to melt the metal powders but this could also causes caking or solid 
state sintering in the powder bed (see section 2.3.3.2). Further investigation is required into the identification of suitable bed temperature for gold powder which could reduce the energy required to melt the gold power.

\subsubsection{Layer Deposition with the New Setup}

Figure 7-17 shows the layers deposited with the new deposition system onto the new build platform. Visual inspection showed that the layers were uniform and the process was repeatable.

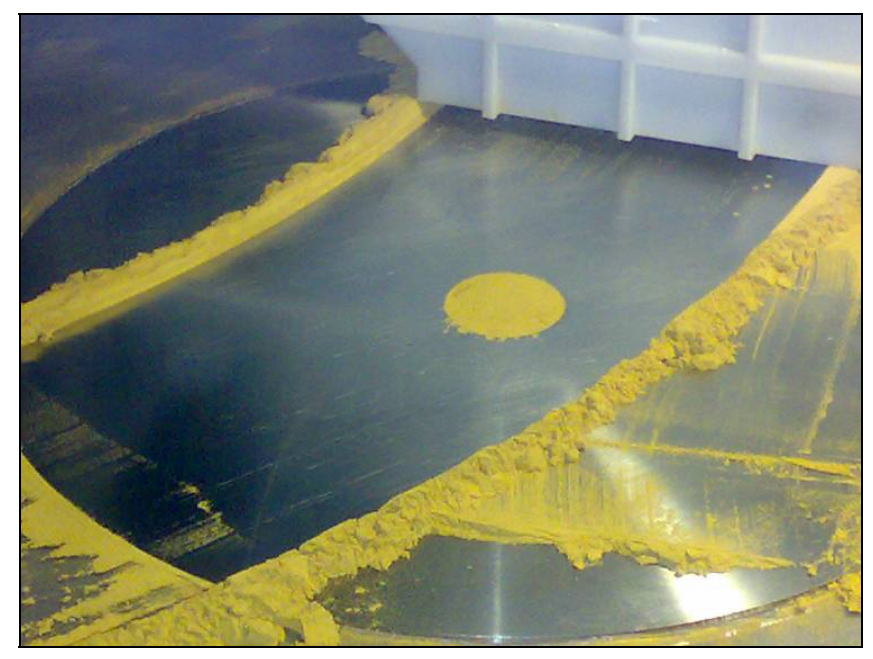

Figure 7-17: Layer deposition with the new deposition system and build platform

\subsection{Summary}

This chapter presented features of the processing system employed in this research work. Material, laser, scan and environmental parameters were discussed in detail. Measurements of the laser spot size were done for different heights of the platform from the bed position both in positive and negative $\mathrm{Z}$ directions. The smallest spot size was found to be $34.5 \mu \mathrm{m}$ for the lowest possible laser power of $3.9 \mathrm{~W}$. Laser optical output power was then measured for different values of input power and current.

The existing powder deposition and build platform was modified to enable processing using a small amount of gold powder. Three different hoppers were designed specially for gold powder and the total build area was reduced to $\phi 15 \mathrm{~mm} \times 20 \mathrm{~mm}$ by making a new build platform. The new build platform optimized the actual material usage in the experimental work and helped reduce material wastage.

The Next chapter explains the optimization of SLM process in detail. 


\section{Chapter 8}

\section{SELECTIVE LASER MELTING (SLM) OF GOLD POWDER}

This chapter discusses the experimental work carried out to identify suitable processing parameters for SLM of gold powder. Single scans, single layers and multiple layers were produced from the gold powder.

\subsection{Single Line Scans}

The first scan track in the SLM is attached to either a substrate. This section presents the experimental results for single scans and single layers of gold powder sintered onto a substrate. By making scan tracks onto a substrate, an extra factor was added to the parameter set i.e. the layer thickness (Lt) of the powder. A steel substrate plate was used and the gold powder was processed with the SLM 100 machine. The parameters involved in single scans were the laser power, powder layer thickness and scan speed. A layer thickness of $100 \mu \mathrm{m}$ was manually deposited for single line and single layer scans experiments which are discussed in this chapter. The oxygen content inside the chamber was always kept below $0.5 \%$ which further decreased during the operation by continuous pumping of argon. Table 8-1 shows the processing parameters and their values for processing the gold powder. A full factorial design (varying each variable over the full range) was used for single scan experiments. Each of the scans was $50 \mathrm{~mm}$ in length and three scans were performed for each parameter setting.

\begin{tabular}{|c|c|c|}
\hline S/No & Parameters & Values \\
\hline 1 & Laser Power & $10,15,20,25,30,35,40,45$ and $50 \mathrm{~W}$ \\
\hline 2 & Scan Speed & $\begin{array}{r}10,15,25,35,45,55,65,75,100,130,150,160, \\
200,250,300,350,400,450 \text { and } 500 \mathrm{~mm} / \mathrm{s}\end{array}$ \\
\hline 3 & Layer thickness & $100 \mu \mathrm{m}$ \\
\hline 4 & Bed temperature & $100^{\circ} \mathrm{C}$ \\
\hline
\end{tabular}

Table 8-1: Laser parameters and their range for single scan experiments

Laser power below 10W was not selected because gold powder could not be melted below this power, whereas $50 \mathrm{~W}$ was the maximum power of the fibre laser installed in the SLM 100 system. The effect of scan speed on the laser processing of gold powder was 
investigated by performing a first set of experiments for a broad range of scan speeds from 15 to $500 \mathrm{~mm} / \mathrm{s}$ with intermediate values of $35,55,75,100,150,200,300$ and $400 \mathrm{~mm} / \mathrm{s}$ for the laser power values specified in Table 8-1. Figure 8-1 shows the processing results of gold powder obtained for these parameter settings. 


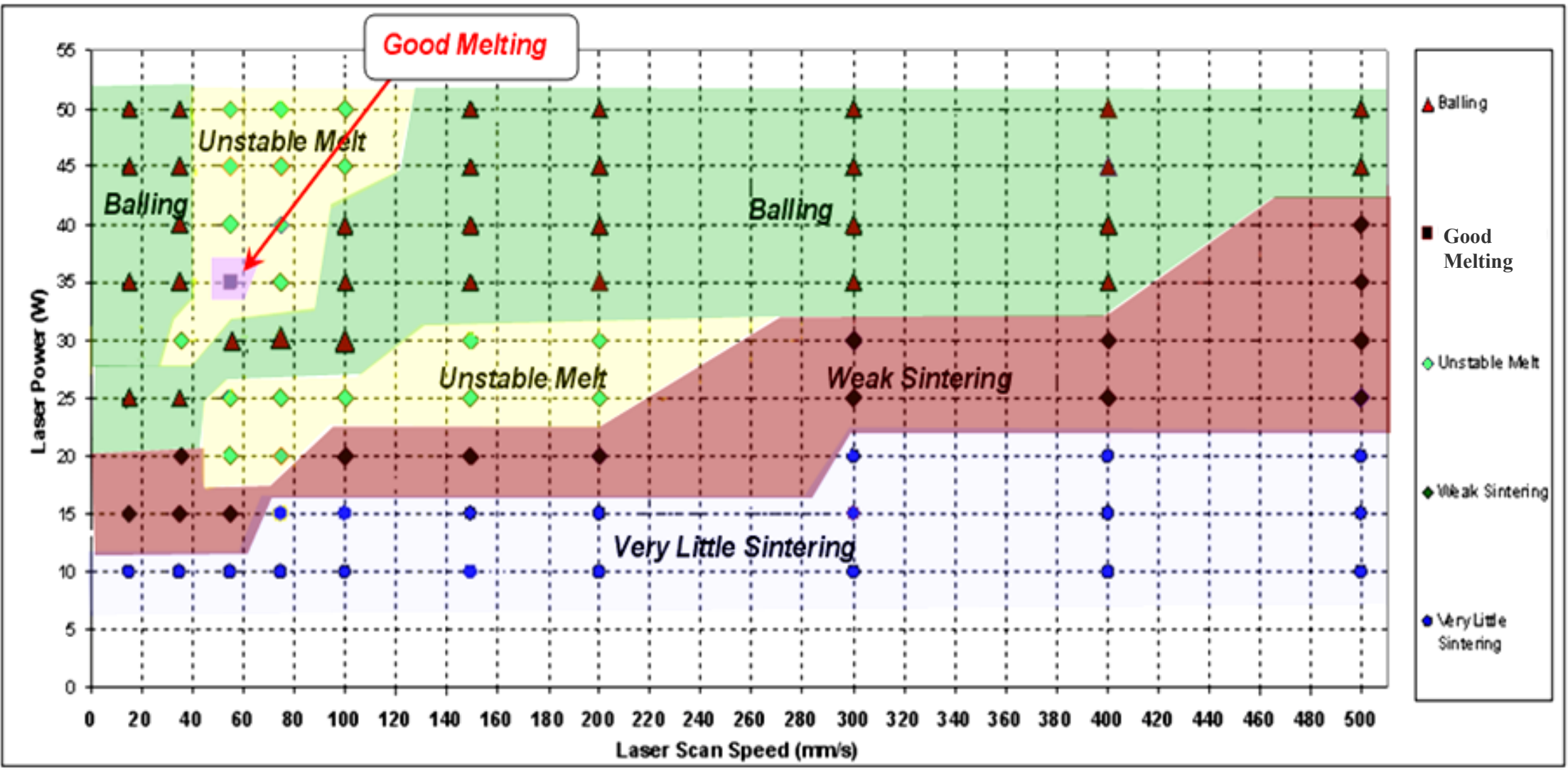

Figure 8-1: Different processing regions for SLM of gold powder for first set of experiments 
Figure 8-1 shows good melting at only a single processing parameter setting within the unstable melt region. Although the gold powder was completely melted in the unstable melt region, the results were different from the good melting region. In the good melting region, a good continuous bead was obtained but the unstable melt region had irregular and discontinuous beads with metal spreading in different directions (explained in detail in section 8.1.2 and 8.1.3). Due to the melting of gold in the unstable melt region, the identification of a good melt region and the tendency of molten metal to form beads, the region between the scan speed of 15 to $200 \mathrm{~mm} / \mathrm{s}$ and laser power of 10 to $50 \mathrm{~W}$ was further investigated by performing a second set of experiments. The second set of experiments was performed for scan speed values of $10,25,45,65,130$ and $160 \mathrm{~mm} / \mathrm{s}$ for laser power values specified in Table 8-1. These experiments were performed in such a way to fill the large gaps between the points in Figure 8-1 and to further understand these regions. Some selected experiments were also performed for scan speed of 250,350 and $450 \mathrm{~mm} / \mathrm{s}$ for the laser power of 25 to $50 \mathrm{~W}$ to further investigate the processing window for high scan speeds. These additional experiments were useful in obtaining a detailed processing window which is shown in Figure 8-2 (with all the experiments).

The different values of laser power and scan speed listed in Table 8-1 were also selected in such a way as to obtain a detailed processing window with a relatively small number of experiments (keeping in view the small amount of material available). A bed temperature of $100^{\circ} \mathrm{C}$ was used for the single scan experiments because the new build platform could only be heated to this temperature as explained in section 7.5.7.

Five different regions were identified in the processing of gold powder as shown in Figure 8-2. These regions are:

- Balling Region

- Good Melting Region

- Unstable Melt Region

- Weak Sintering Region and

- Very Little Sintering Region

Each of these regions are explained in detail next in this chapter. 


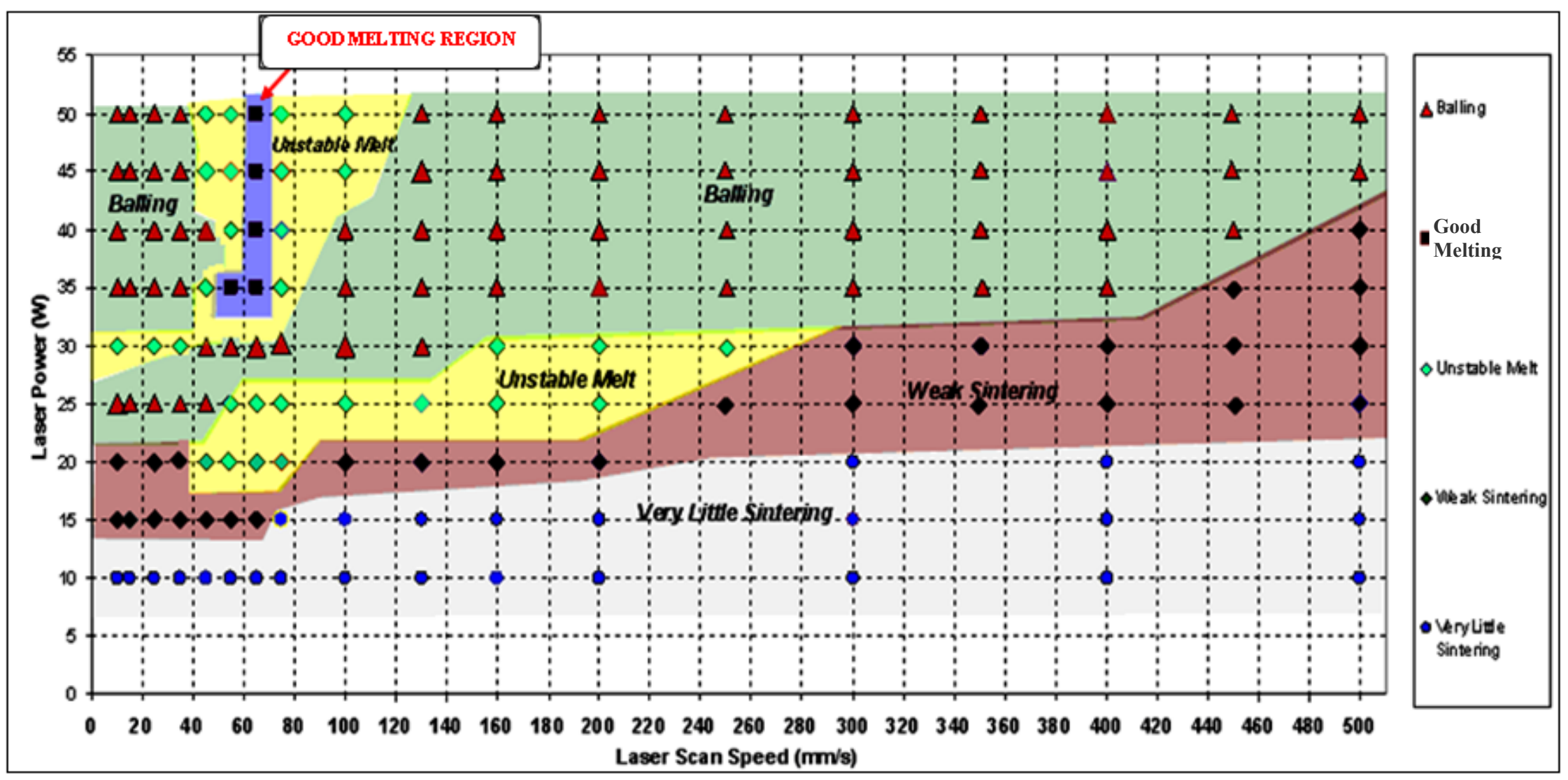

Figure 8-2: Complete processing window for SLM of gold powder 


\subsubsection{Balling Region}

The balling phenomenon was observed within two different regions in the processing window (Figure 8-2) of gold powder. Figure 8-3 shows an image of the balling in a single scan. The balling phenomenon was observed at a laser power above $20 \mathrm{~W}$.

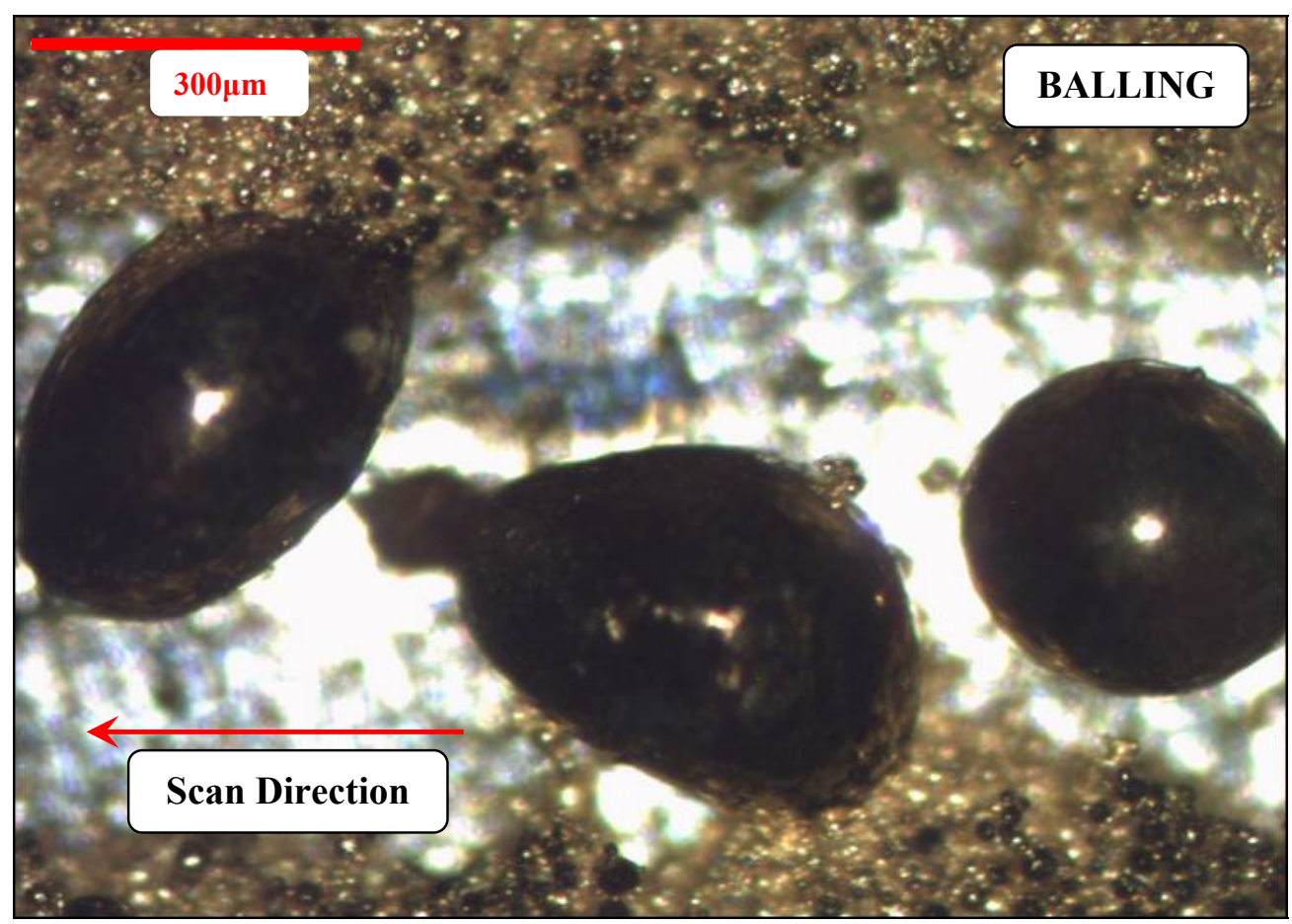

Figure 8-3: Balling at 50W laser power and $25 \mathrm{~mm} / \mathrm{s}$ scan speed

At low scan speeds, the molten material remains in the molten state for much longer as compared to higher scan speeds. Therefore, a possible explanation for the balling at low scan speeds in the processing window (Figure 8-2) could be the high energy input and more time available for the molten metal to split into droplets before it could resolidify. The time available for the molten metal to split into droplets before solidification is called the break-up time.

At higher scan speeds and higher laser power, the balling could be due to an increase in the length and decrease in the width of the scan tracks causing the L/D ratio to rise above 2.1, thus destabilizing the melt pool. Rombouts et al (2006) used a CMOS high speed camera and also observed that an increase in scan speed (from $6 \mathrm{~mm} / \mathrm{s}$ to $50 \mathrm{~mm} / \mathrm{s}$ ) increases the melt pool size, mainly the melt pool length while its width remains almost the same. In both the cases of low and high scan speed induced balling, the non-adhesion of 
molten gold to the substrate also promoted balling as discussed for other materials elsewhere (O'Neill et al, 1998).

Figure 8-4 shows the balling at different laser power and scan speeds. It is quite evident that a high laser power combined with a low scan speed $(50 \mathrm{~W}$ and $25 \mathrm{~mm} / \mathrm{s})$ results in the formation of larger droplets. The size of the droplets tends to increase with decreasing scan speeds and increasing laser power. This could be related to the break-up time of the molten metal. This is the time available for the molten metal to split into droplets before the re-solidification could occur. Rombouts et al (2006) discussed the time for break-up of the melt pool to occur and suggested that an increase in break up time results in the formation of larger droplets which are far apart from each other. Although an increase in the droplet size was observed with decreasing scan speed and increasing power, no clear trend could be observed for the change in the gaps between these droplets. 

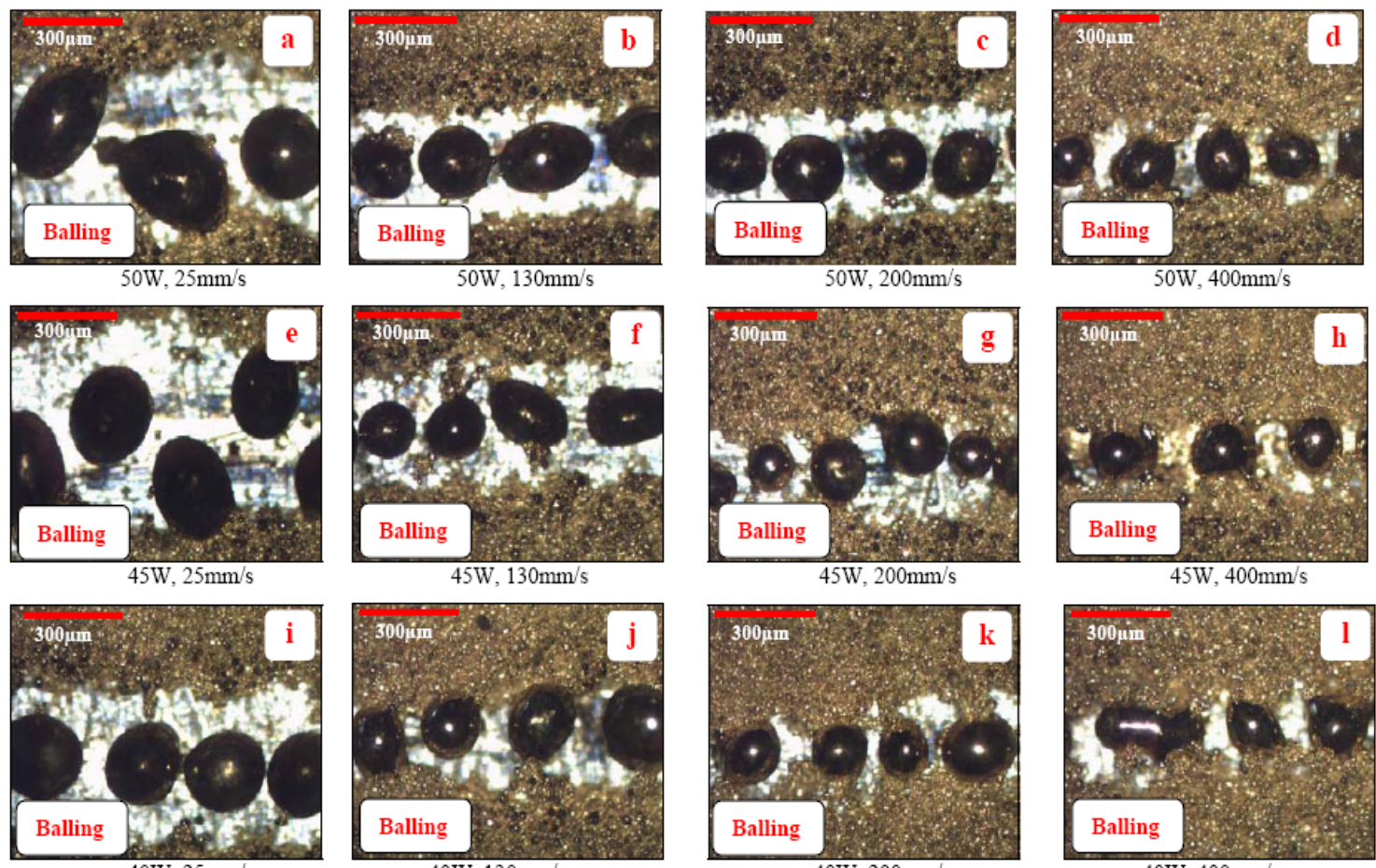

$40 \mathrm{~W}, 130 \mathrm{~mm} / \mathrm{s}$
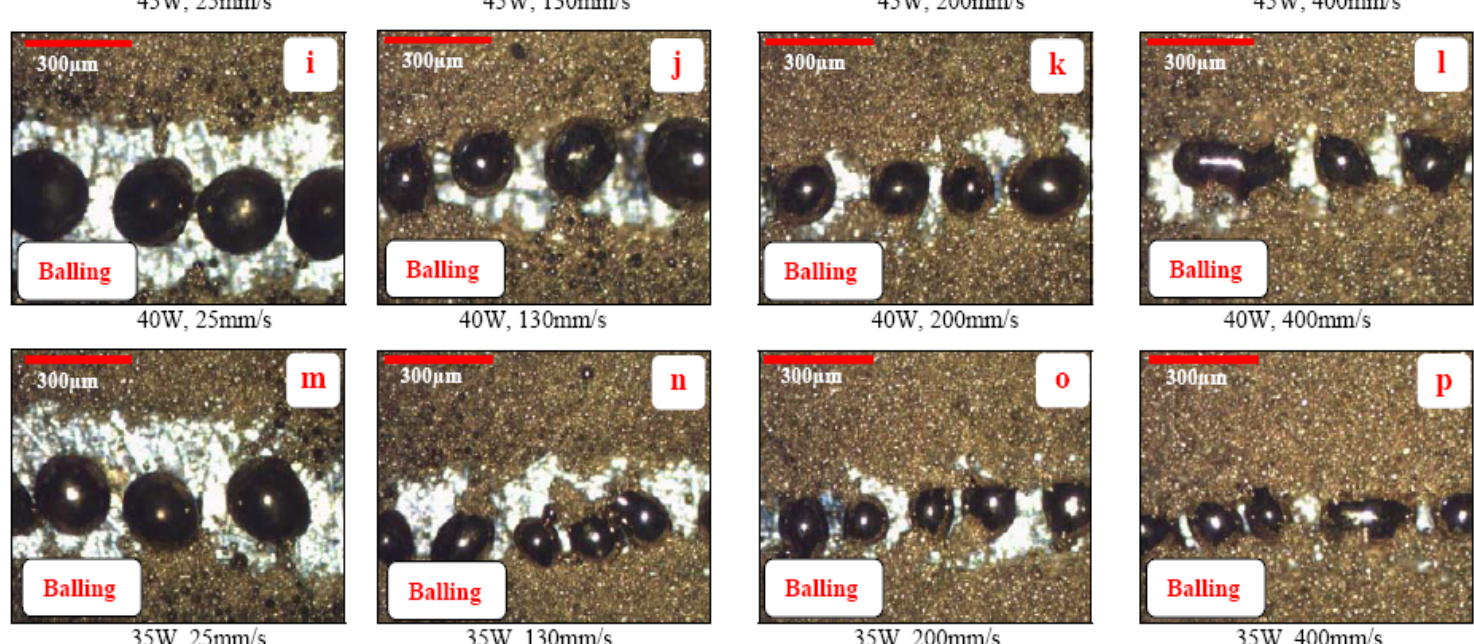

$40 \mathrm{~W}, 400 \mathrm{~mm} / \mathrm{s}$
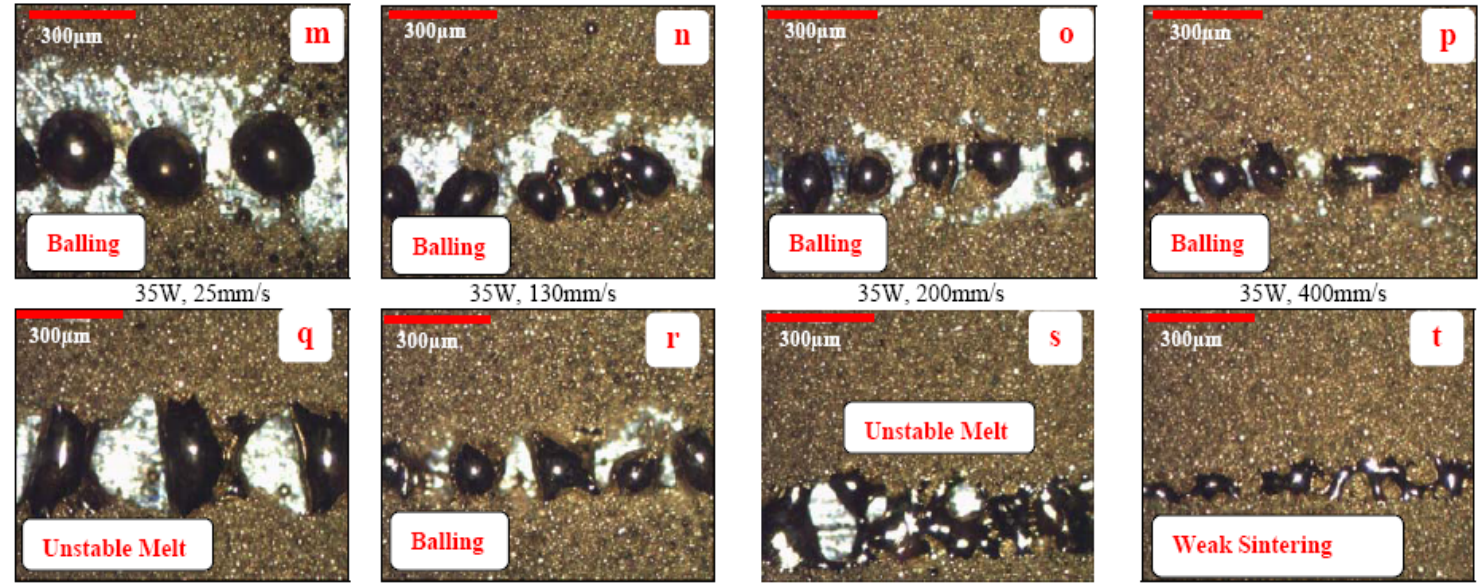

$30 \mathrm{~W}, 130 \mathrm{~mm} / \mathrm{s}$

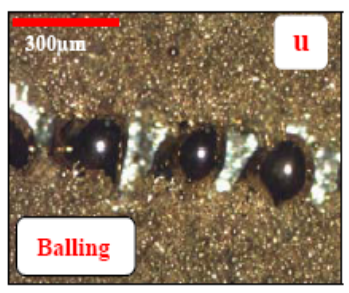

$25 \mathrm{~W}, 25 \mathrm{~mm} / \mathrm{s}$

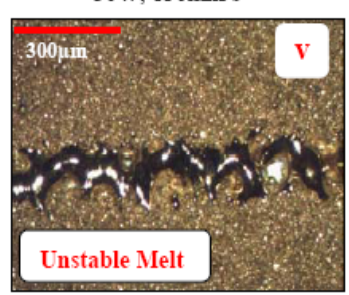

$25 \mathrm{~W}, 130 \mathrm{~mm} / \mathrm{s}$

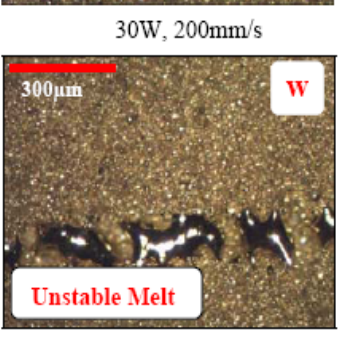

$25 \mathrm{~W}, 200 \mathrm{~mm} / \mathrm{s}$

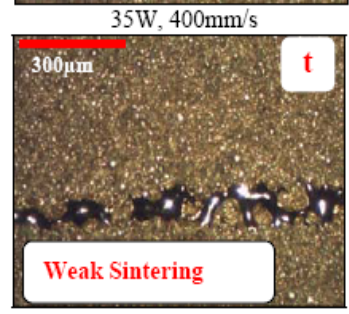

$30 \mathrm{~W}, 400 \mathrm{~mm} / \mathrm{s}$

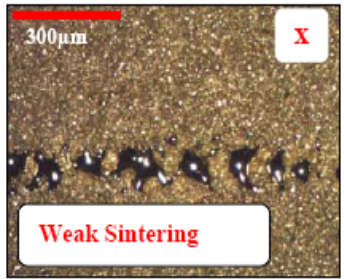

$25 \mathrm{~W}, 400 \mathrm{~mm} / \mathrm{s}$

Figure 8-4: (a-x) Balling, unstable melt and weak sintering at different laser power and scan speeds (all images were taken at same magnification) 
In Figure 8-4, within a row of constant laser power, at lower scan speeds there is less gold powder present on both sides of the droplets than at high scan speeds. This was observed for all of the laser power settings (from left to right within all the rows) presented in Figure 8-4. A possible explanation for this could be that at lower scan speeds the melt pool width is increased and the laser stays at a point for a longer period of time thus the gold powder present on both sides of the path of laser scan has enough time to melt and move towards the melt pool resulting in larger melt pool and larger droplets formation after the balling occurs. Conversely, at high scan speeds, the melt pool width is relatively small and the laser passes very quickly over a point on the laser path. Therefore, gold powder present on both sides of the laser path does not have enough time and energy available to melt and move towards the melt pool resulting in more un-melted gold powder available on both sides of the droplets at these scan speeds.

Kruth et al (2004) used an iron-based powder to identify the processing window with a laser power of 70 to $200 \mathrm{~W}$ and scan speed of 20 to $400 \mathrm{~mm} / \mathrm{s}$. Niu and Chang (1999) processed High Speed Steel (HSS) powder in the laser power range of $2.5 \mathrm{~W}$ to $150 \mathrm{~W}$ and scan speed of 5 to $22 \mathrm{~mm} / \mathrm{s}$. Both of these cases observed balling phenomena as seen in this research, but due to the difference in the processing ranges a generalised conclusion could not be deduced for the balling phenomena at low or high laser power and scan speed combinations.

\subsubsection{Good Melting Region}

From the processing window of gold (Figure 8-2), a very narrow good melting region was identified. Figure 8-5 shows an image of the single scan from the good melting region. The good melting region showed no signs of balling and single beads of molten gold were successfully obtained. Although the lines produced had non-uniform edges and the width was also non-uniform, the gold powder was found to be fully melted in this region. The non-uniform edges of these lines could be due to the outward flow of molten metal from the path of the laser scan owing to the negative surface tension and thermal gradients in the melt pool as discussed in section 2.3.3.3. Kruth et al (2004) observed that the smooth and regular region gets wider as the laser power is increased form 70 to $200 \mathrm{~W}$ during the Continuous Wave $(\mathrm{CW})$ laser processing of iron based powder. This could also be true for gold powder where the processing window (Figure 8-2) was only obtained for a maximum laser powder of $50 \mathrm{~W}$ and this could be the possible explanation for a narrow 
good melting region observed in Figure 8-2. Further investigation is needed to expand the current processing window (Figure 8-2) for higher laser power (above 50W).

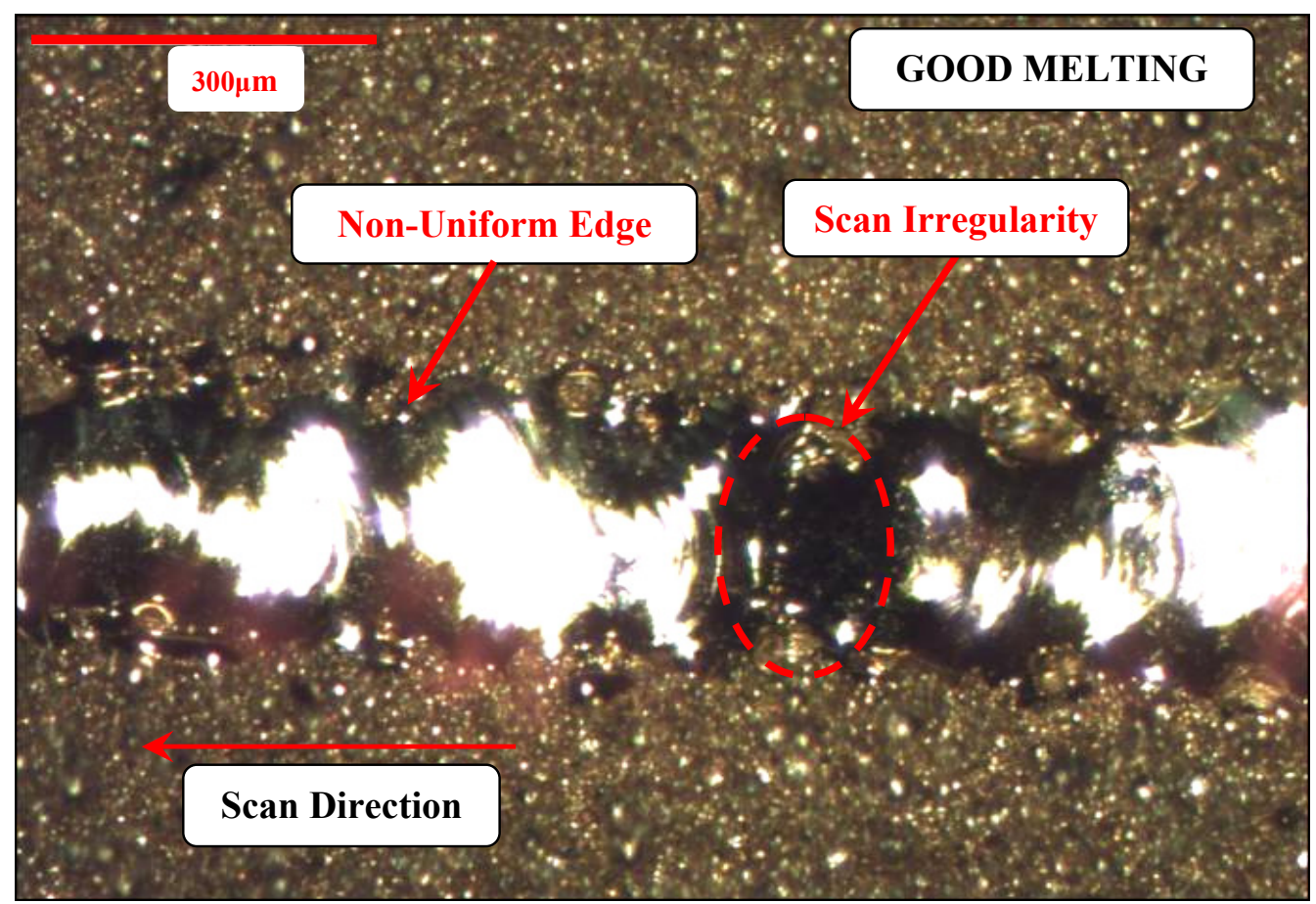

Figure 8-5: Good melting at $50 \mathrm{~W}$ laser power and $65 \mathrm{~mm} / \mathrm{s}$ scan speed

\subsubsection{Unstable Melt Region}

Two regions of unstable melt were also identified where the melt pool solidified to form an irregular shape. Figure 8-6 shows an unstable melt scan. In this region, gold powder was completely melted but liquid metal tended to spread on the surface of the substrate irregularly thus not creating stable beads. This region was not included in the good melting region because the uneven melting of the gold could reduce part accuracy and result in non-uniform layers, internal part porosity and problems during the deposition process. The unstable melt region at high laser power (30 to $50 \mathrm{~W})$ which was present on both sides of the good melting region represented an intermediate state between the good melting region and the balling phase. In this intermediate unstable melt region the gold powder had been fully melted but the melt pool solidified before either it could form continuous beads or the balling could occur, thus resulting in an irregular solidified state.

The unstable melt region was also observed for a laser power of 20 to $30 \mathrm{~W}$ and scan speed of 45 to $200 \mathrm{~mm} / \mathrm{s}$. This region of unstable melt occurred in between the weak sintering and the balling region. The possible reason for this unstable melt region to occur could be that the gold powder was melted more than in the weak sintering region thus 
forming a relatively smaller melt pool but the material in the melt pool was not enough for it to split up and form balling. This resulted in the solidification before complete splitting of the melt pool.

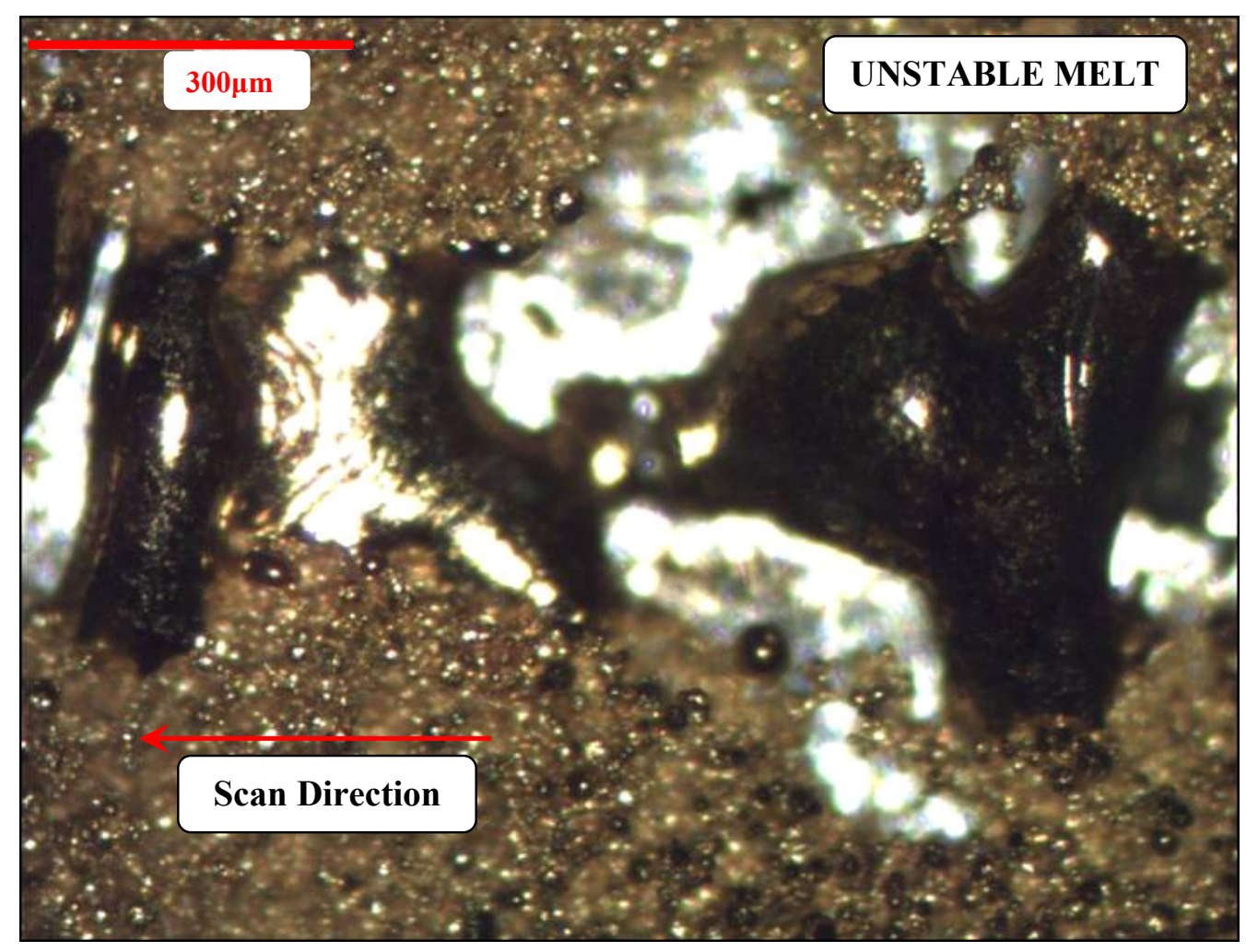

Figure 8-6: Unstable melt at $50 \mathrm{~W}$ laser power and $45 \mathrm{~mm} / \mathrm{s}$ scan speed

\subsubsection{Weak Sintering Region}

The weak sintering region is differentiated from the very little sintering region by a small melt pool formation which was not observed in the very little sintering region as shown in Figure 8-7. The weak sintering region occurred for low laser power (15-20W) at low scan speeds (10 to $200 \mathrm{~mm} / \mathrm{s}$ ) and expanded for high laser power (20 to $40 \mathrm{~W}$ ) at high scan speeds (200 to $500 \mathrm{~mm} / \mathrm{s}$ ). Because of the high reflectivity of the gold powder, the gold powder did not receive enough energy in this region to form a larger melt pool. The weak sintering region could not produce continuous gold beads. 


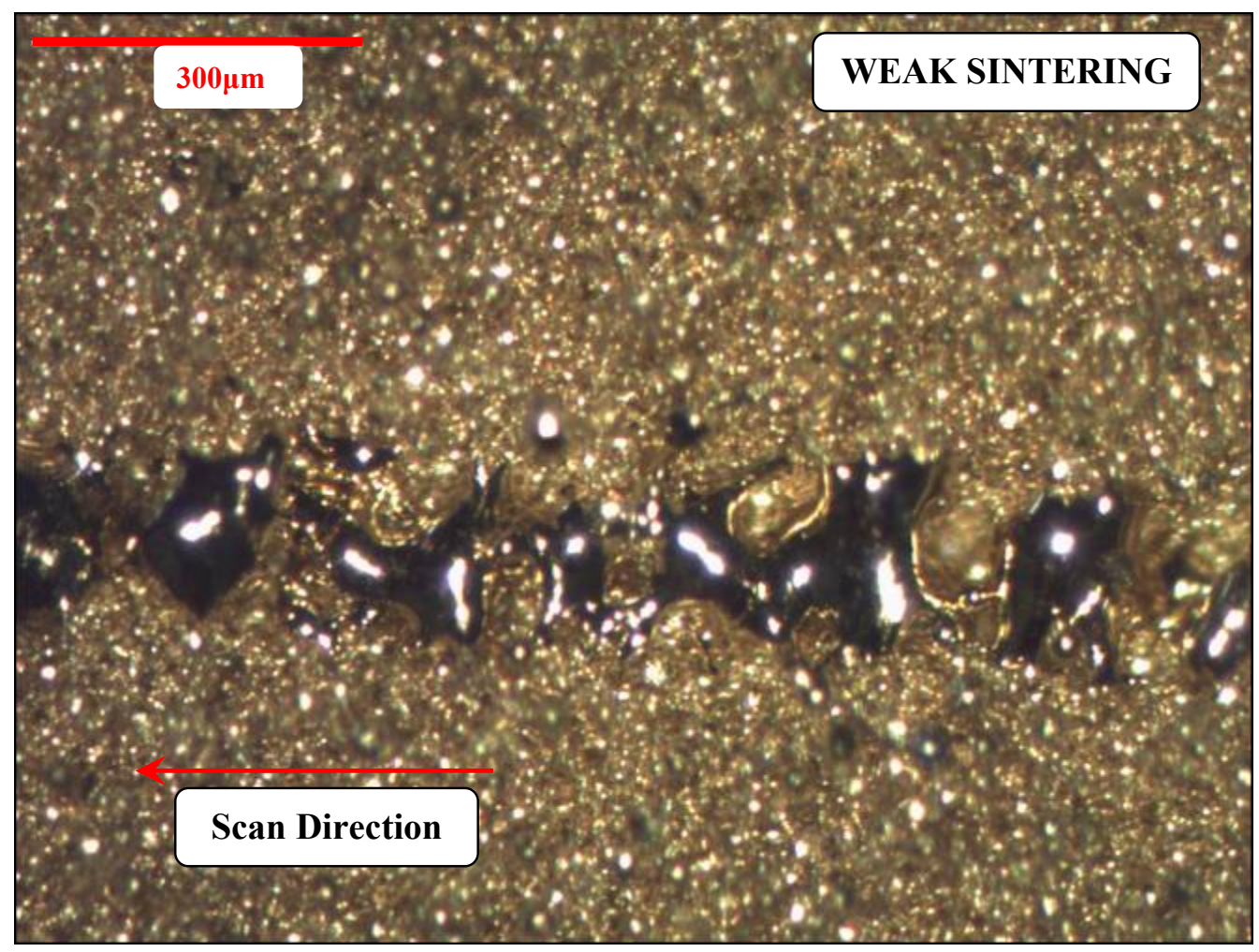

Figure 8-7: Weak sintering at $15 \mathrm{~W}$ laser power and $25 \mathrm{~mm} / \mathrm{s}$ scan speed

\subsubsection{Very Little Sintering Region}

Similar to the weak sintering region, due to the high reflectivity of gold powder and low laser power, this region showed very little sintering of the gold powder. This region is different from the weak sintering region in that there seems to be very little melt pool formation; rather gold powder is melted at various isolated points in the path of the laser scan. Figure 8-8 shows a single scan in the very little sintering region. 


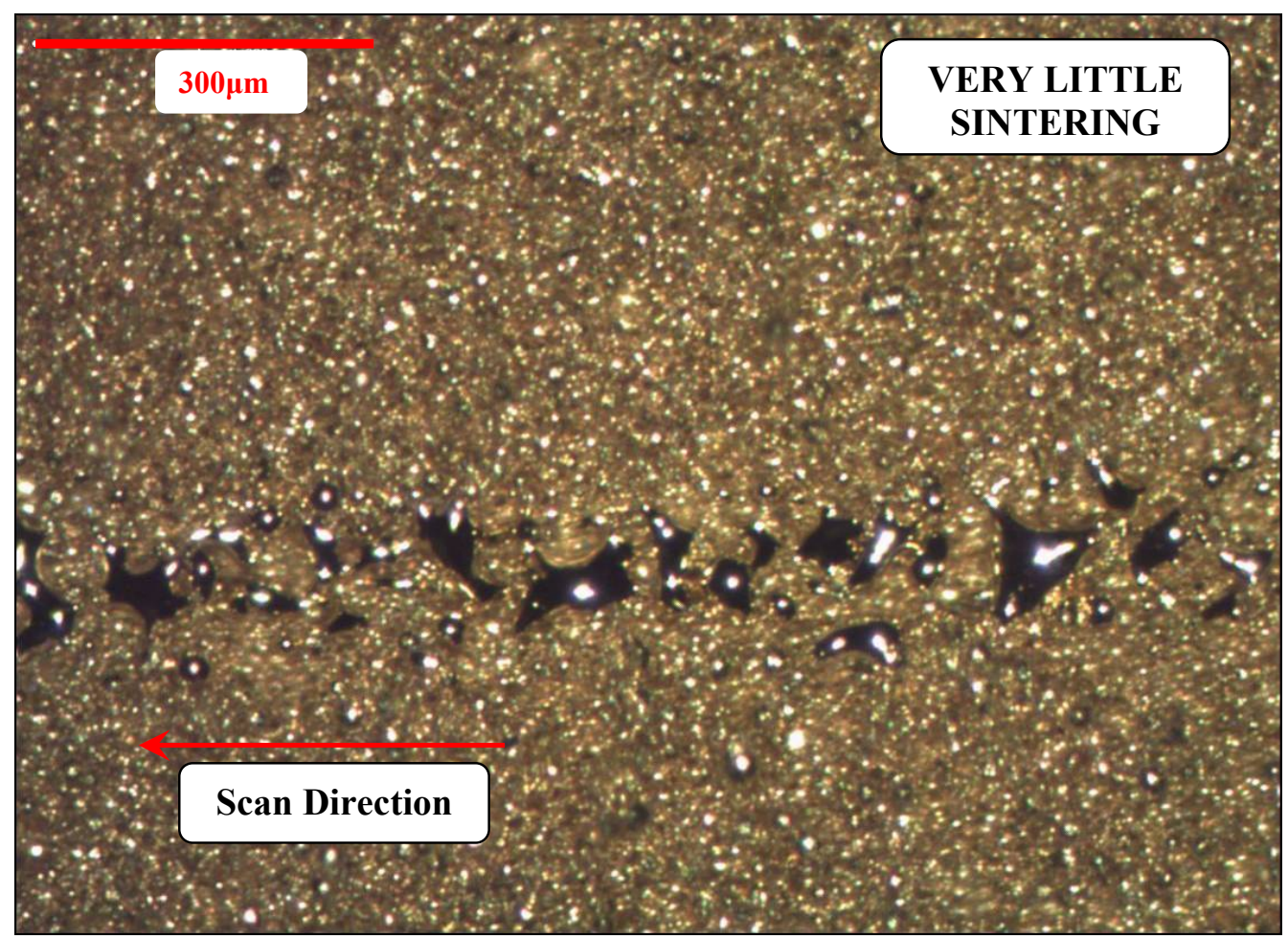

Figure 8-8: Very little sintering at $10 \mathrm{~W}$ laser power and $10 \mathrm{~mm} / \mathrm{s}$ scan speed

\subsection{Splitting of the Melt Pool}

Another phenomenon which was observed during the processing of gold powder was splitting of the melt pool away from the path of the laser scan. Figure 8-9 shows the splitting of the melt pool and presence of solidified droplets on both sides of the laser path. This mainly occurred at low scan speeds and low laser power (25W and 10 to $45 \mathrm{~mm} / \mathrm{s}$ ) within the balling region. The possible explanation for this could be the presence of a negative surface tension gradient within the melt pool. Pure metals and many alloys are already known to have a negative surface tension gradient (Rombouts et al, 2006). This negative surface tension gradient induces an outward flow of molten metal from the centre of the melt pool (Beer and Ring, 1972). As the melting takes place on a solid substrate to which the gold has almost no adhesion, the beads produce balls which move away from the path of the laser, thus creating large droplets on both sides of the laser scan path. 


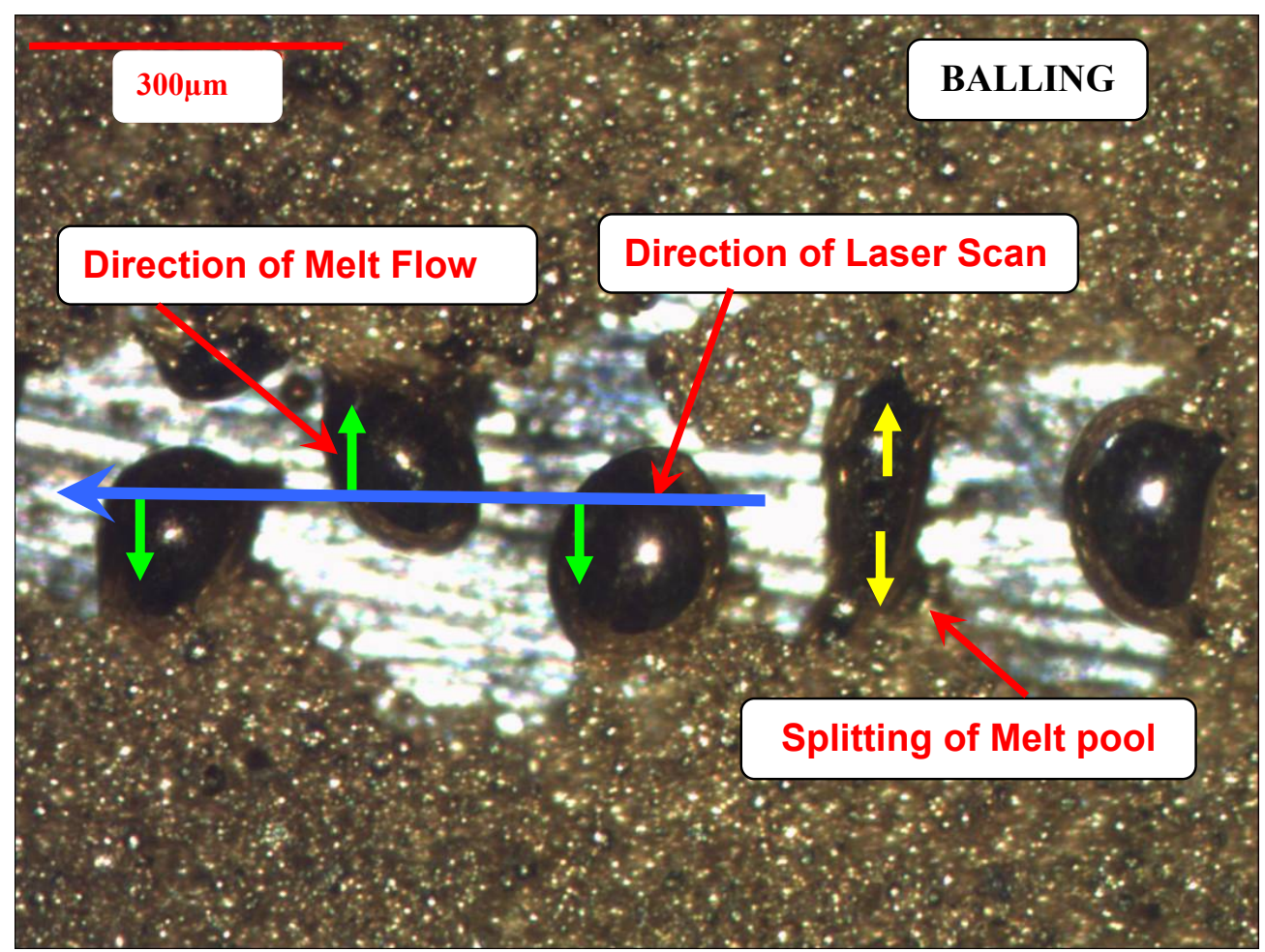

Figure 8-9: Microscope image of single line scan of gold: melt pool splitting at 25W laser power and $25 \mathrm{~mm} / \mathrm{s}$ scan speed

Another possible explanation for splitting of the melt pool could be the heat loss from the melt pool. Heat is dissipated in three ways from the melt pool i.e. heat loss to the atmosphere, through the powder layer and to the substrate underneath the melt pool (Figure 8-10). The maximum heat transfer takes place through the substrate (T1) then through the powder layer (T2) and the least through the top surface to the atmosphere, thus creating a temperature gradient as $\mathrm{T} 1>\mathrm{T} 2>\mathrm{T} 3$. In this case the hot metal at $\mathrm{T} 1$ would start flowing towards the sides due to the temperature gradient and as the molten metal is not fused to the underlying substrate at this stage, the melt pool would split into two halves and move away from the path of laser scan.

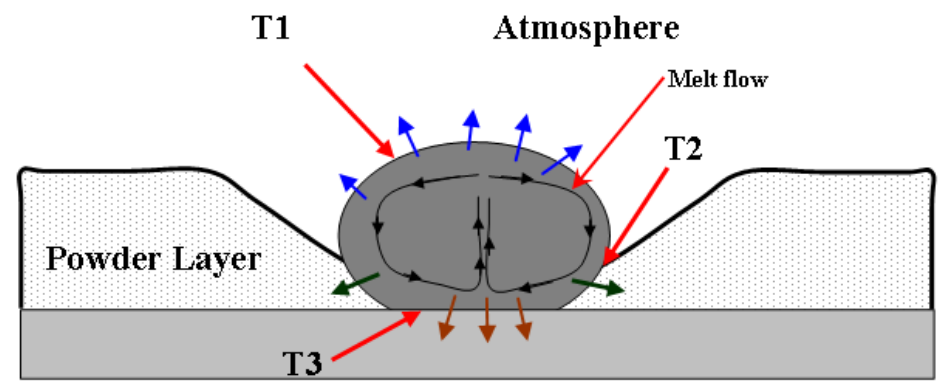

Figure 8-10: Heat transfer and temperatures during the laser melting process 


\subsection{Single Layer Scans}

In the previous section, single scans were performed on the substrate with a $100 \mu \mathrm{m}$ layer of gold powder. The main aim of producing single layers in this section was to check the quality of the layers produced with the parameters selected from different processing regions i.e. balling, good melting, unstable melt, weak sintering and very little sintering regions as shown in Figure 8-2. As clear from the single scan experiments, good bead formation of gold was achieved at relatively high laser power and low scan speeds i.e. towards the upper left corner of the graph in Figure 8-2. Due to the small amount of material available, single layers could not be produced for each of the parameter settings shown in Figure 8-2. Therefore, the parameters were selected for the single layer experiments from the aforementioned regions in such a way as to achieve optimum representation of each of the regions. Hence the single layer scans were performed for the parameters falling in the beginning, middle and high end of each of the regions. Table 8-2 shows the 17 different parameters settings selected for 20x20 mm single layers produced in this section.

\begin{tabular}{|c|c|c|}
\hline Laser Power (W) & $\begin{array}{c}\text { Scan Speeds } \\
(\mathrm{mm} / \mathrm{s})\end{array}$ & Processing Region \\
\hline 40 & 200 & Balling \\
\hline 35 & 25 & Balling \\
\hline 45 & 400 & Balling \\
\hline 25 & 25 & Balling \\
\hline 50 & 25 & Balling \\
\hline 35 & 65 & Good Sintering \\
\hline 50 & 65 & Good Sintering \\
\hline 25 & 160 & Unstable Melt \\
\hline 45 & 100 & Unstable Melt \\
\hline 20 & 50 & Unstable Melt \\
\hline 30 & 25 & Unstable Melt \\
\hline 30 & 400 & Weak Sintering \\
\hline 15 & 25 & Weak Sintering \\
\hline 20 & 200 & Weak Sintering \\
\hline 15 & 200 & Very Little Sintering \\
\hline 20 & 400 & Very Little Sintering \\
\hline 10 & 25 & Very Little Sintering \\
\hline
\end{tabular}

Table 8-2: $\quad$ Experimental parameters for SLM of single layers of gold 
A number of single layers were scanned on the gold powder bed to select the hatch distance. These scans were performed at $50 \mathrm{~W}$ laser power and scan speed of $65 \mathrm{~mm} / \mathrm{s}$, which were selected from the good melting region to form continuous beads. It was observed that each scan was approximately $80 \mu \mathrm{m}$ in width and thus a hatch distance higher than $80 \mu \mathrm{m}$ resulted in no scan overlap. Therefore, a hatch distance of $80 \mu \mathrm{m}$ was selected for the single scan experimentation. Figure 8-11 shows single layers produced with a hatch distance of 80 and $100 \mu \mathrm{m}$. The effect of hatch distance on the porosity of gold parts is discussed in detail in Chapter 9.
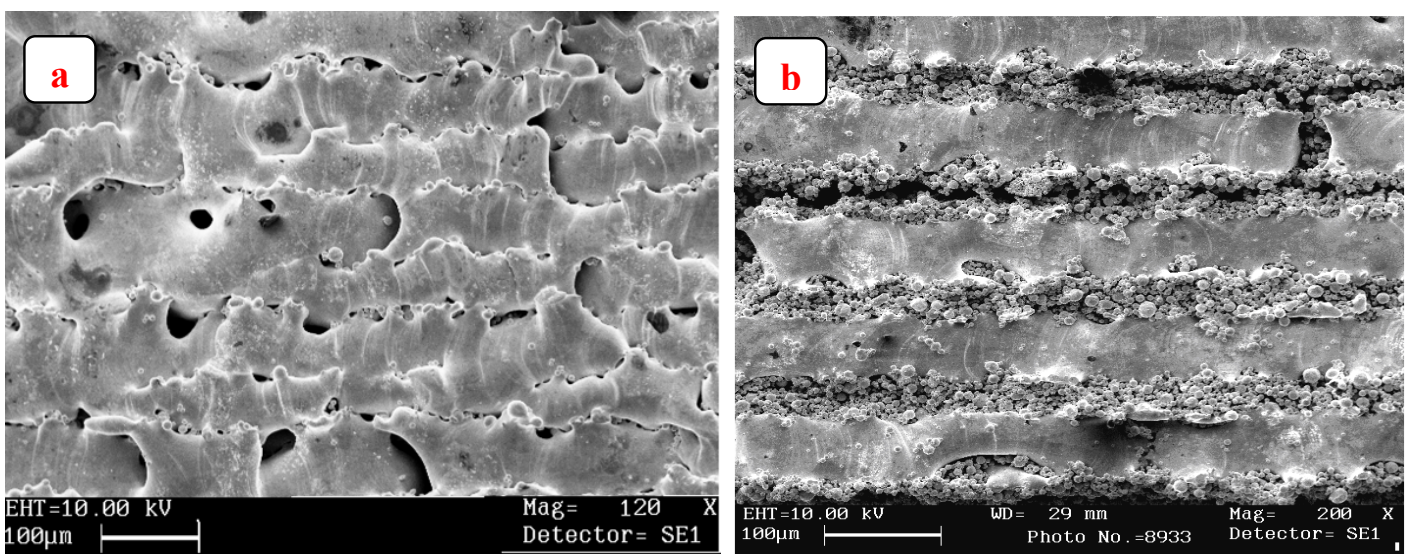

Figure 8-11: Single layers scanned with (a) $80 \mu \mathrm{m}$ hatch distance (b) $100 \mu \mathrm{m}$ hatch distance

A simple scan strategy was employed for single layer scans in order the check the effect of other processing parameters i.e. laser power and scan speed on the single layers. The scan strategy involved full hatching in the area with a single fill contour and boundary scan at the outer contour of the square $(20 \times 20 \mathrm{~mm}$ in size) (Figure $8-12)$. The hatch offset and fill contour offset were maintained at $80 \mu \mathrm{m}$.

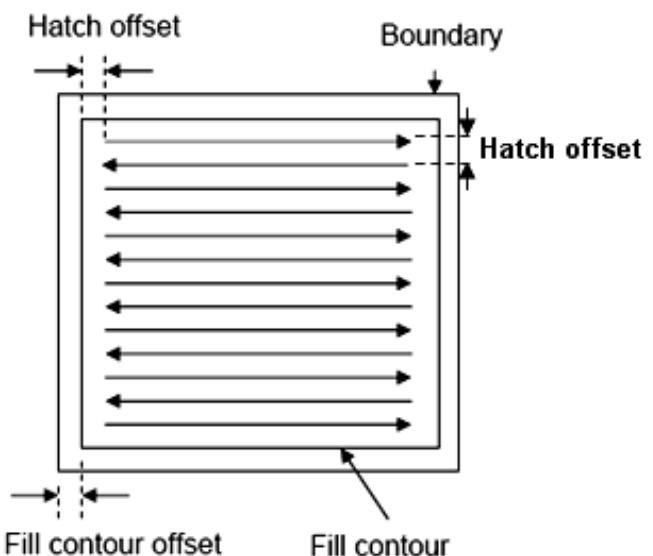

Figure 8-12: Scan strategy for single layer experimentation 
In order to perform the single layer experiments, a single $100 \mu \mathrm{m}$ layer of gold powder was spread manually into a recess in the substrate plate as shown in Figure 8-13. All the single layer experiments were performed at a bed temperature of $100^{\circ} \mathrm{C}$ similar to the single scans. A first line balling phenomenon was also observed in some of the scans which is explained in detail later in this chapter.

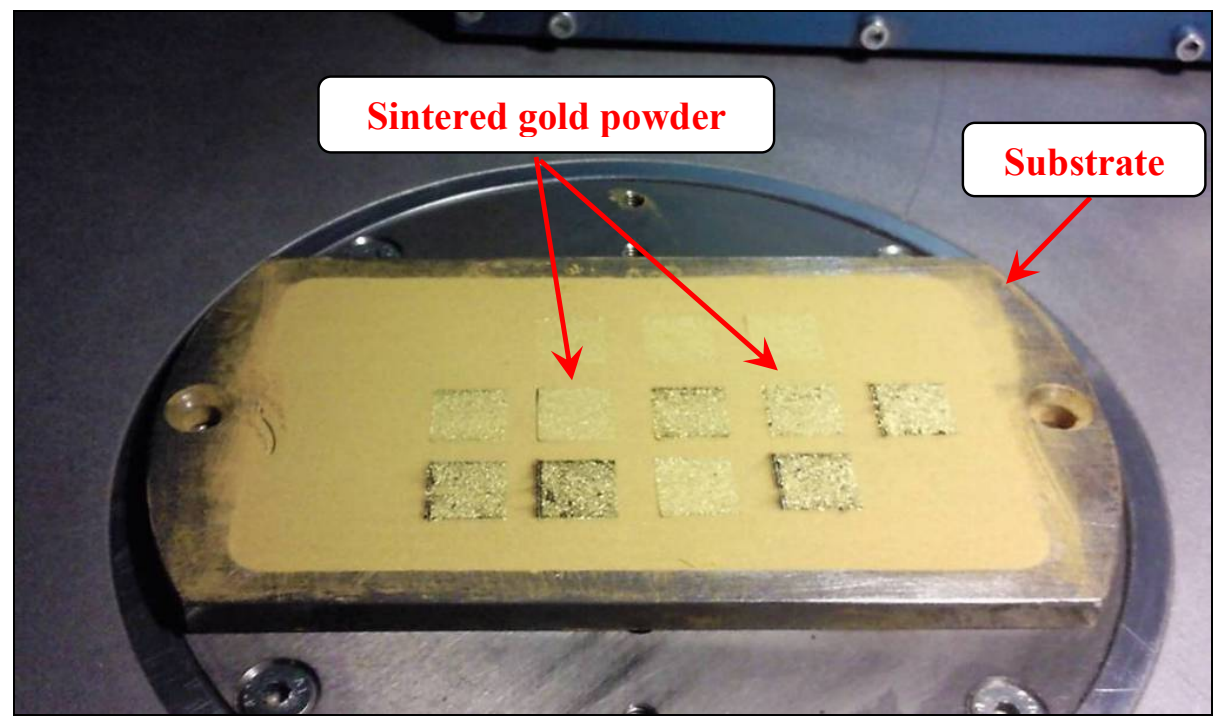

Figure 8-13: Experimental setup for producing single layers of gold

The different parameters selected within a region (shown in Table 8-2) were observed to be similar. One sample from each of these sections is discussed in detail below.

The areas scanned using the balling region parameters $(50 \mathrm{~W}$ and $25 \mathrm{~mm} / \mathrm{s})$ showed complete melting of the gold powder but no clear bead formation (Figure 8-15). There were many places where the molten metal did not wet the underlying substrate completely thus resulting in areas where the underlying substrate could be seen as shown in Figure 814. 


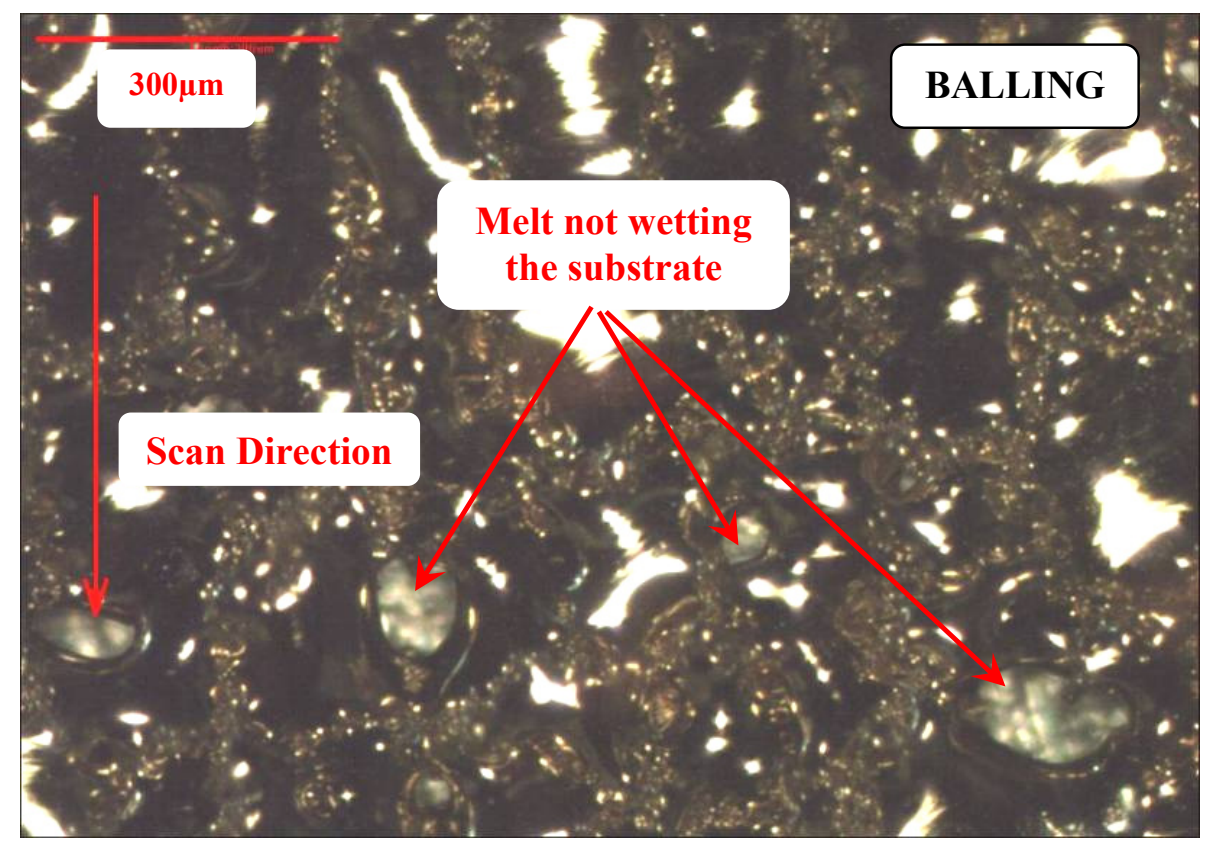

Figure 8-14: Optical microscope image of single layer scan of gold: balling at 50W laser power and $25 \mathrm{~mm} / \mathrm{s}$ scan speed

The single layers sintered with good sintering parameters $(50 \mathrm{~W}$ and $65 \mathrm{~mm} / \mathrm{s}$ ) showed complete melting of the gold powder with clear good bead formation. It can also be seen that in Figure 8-15, the melt did not completely wet the substrate similar to Figure $8-14$, creating areas where the underlying substrate can be seen. This can cause internal porosity in the part which is discussed in Chapter 9.

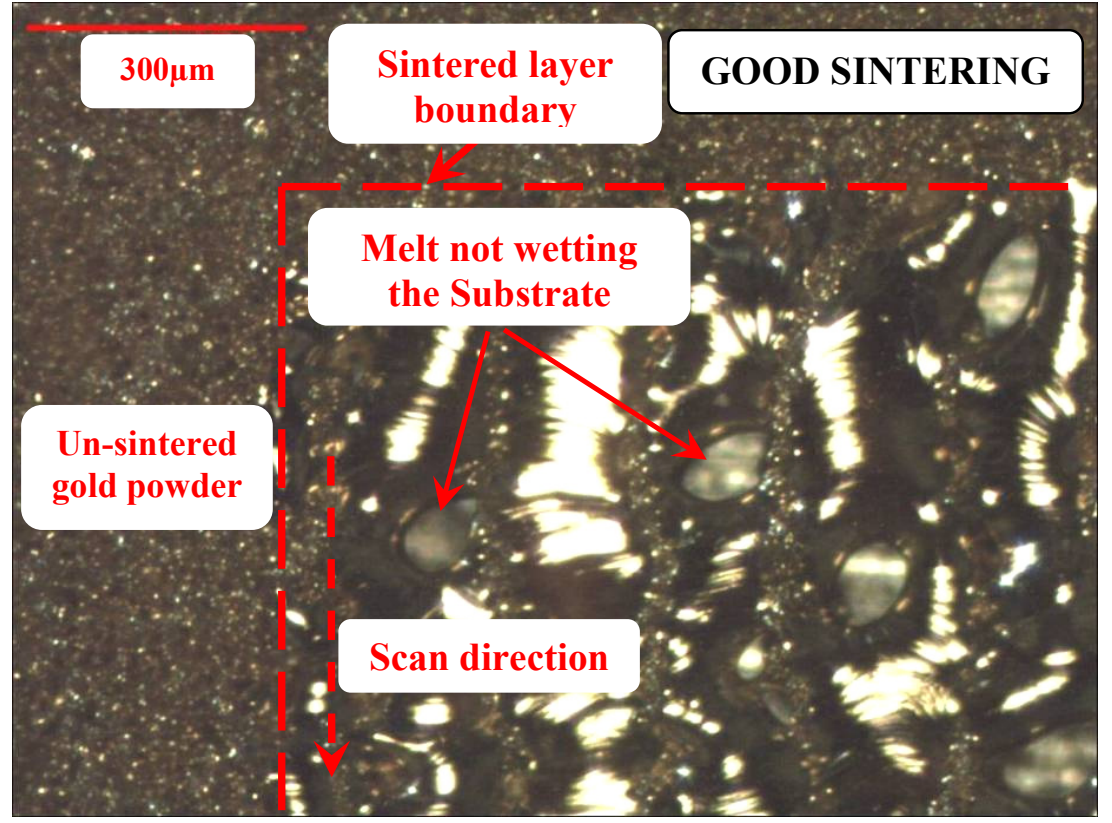

Figure 8-15: Optical microscope image of single layer scan of gold: good sintering at $50 \mathrm{~W}$ laser power and $65 \mathrm{~mm} / \mathrm{s}$ scan speed 
The layers sintered with unstable melt parameters $(45 \mathrm{~W}$ and $100 \mathrm{~mm} / \mathrm{s})$ did melt the gold powder completely but the molten metal spread in different directions resulting in nothing useful as shown in Figure 8-16.

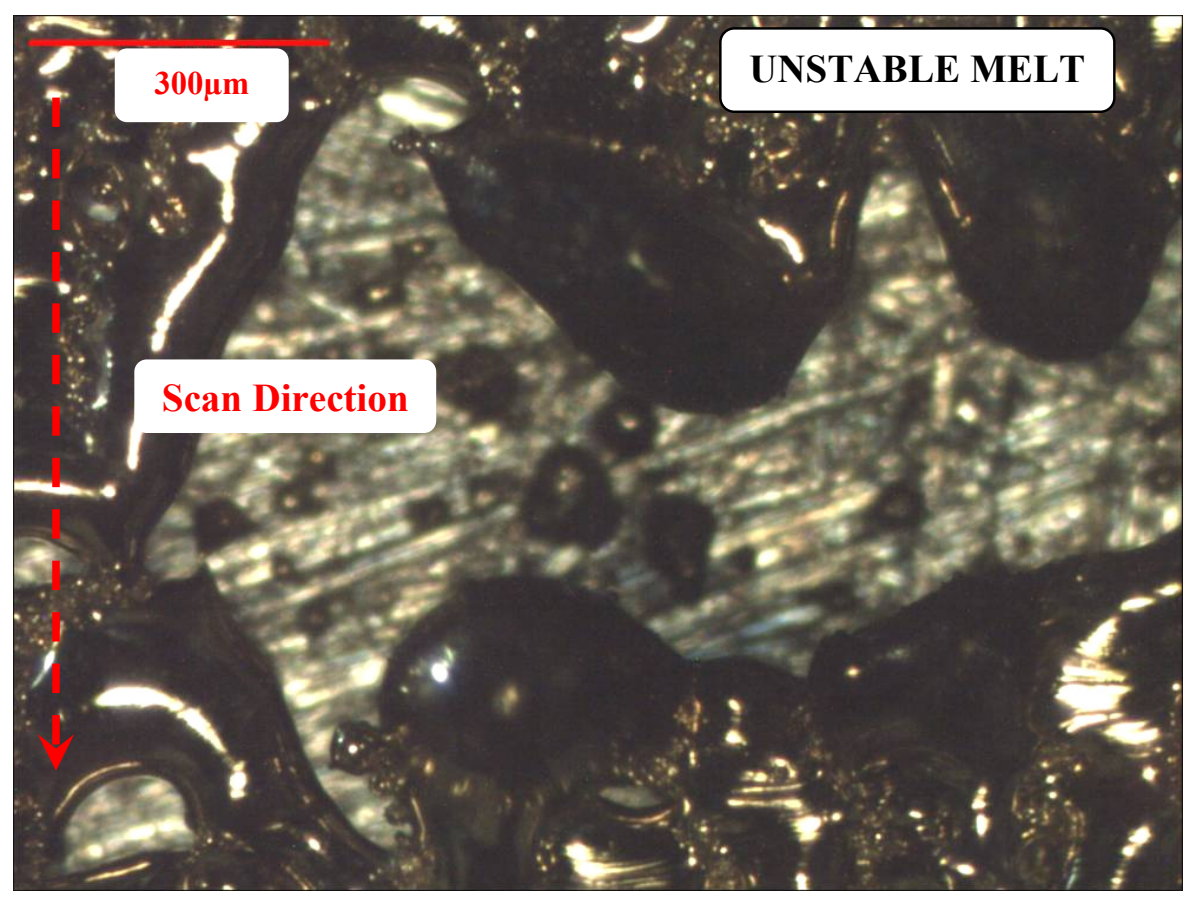

Figure 8-16: Optical microscope image of single layer scan of gold: unstable melt at $45 \mathrm{~W}$ laser power and $100 \mathrm{~mm} / \mathrm{s}$ scan speed

The layers sintered with weak sintering and very little sintering parameters $(15 \mathrm{~W}$, $25 \mathrm{~mm} / \mathrm{s}$ and $10 \mathrm{~W}, 25 \mathrm{~mm} / \mathrm{s}$ respectively) showed partial melting of gold powder at different points across the deposited layer of gold powder. Figure 8-17 and Figure 8-18 shows the single layers processed with the weak and very little sintering parameters

The layers produced with the balling, unstable melt, weak sintering and very little sintering parameters were not found suitable for multiple layer manufacturing either due to the lack of complete melting of gold or discontinuous layers. However, the single layers produced using parameters from the good melting region (50W and $65 \mathrm{~mm} / \mathrm{s}$ ) (Figure 8-15) showed satisfactory results and therefore these parameters were selected for multiple layer manufacturing which is discussed later in this chapter. 


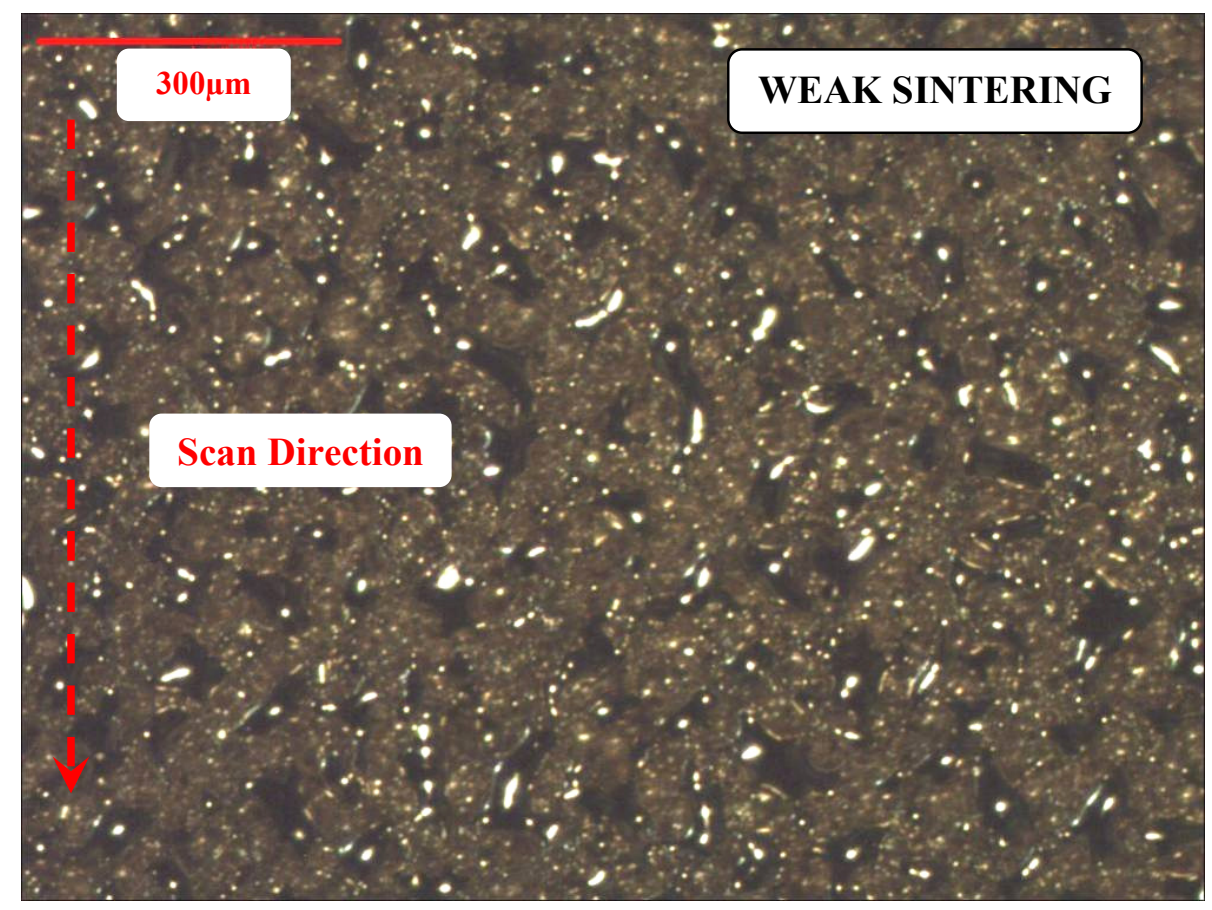

Figure 8-17: Microscope image of single layer scan of gold: weak sintering at $15 \mathrm{~W}$ laser power and $25 \mathrm{~mm} / \mathrm{s}$ scan speed

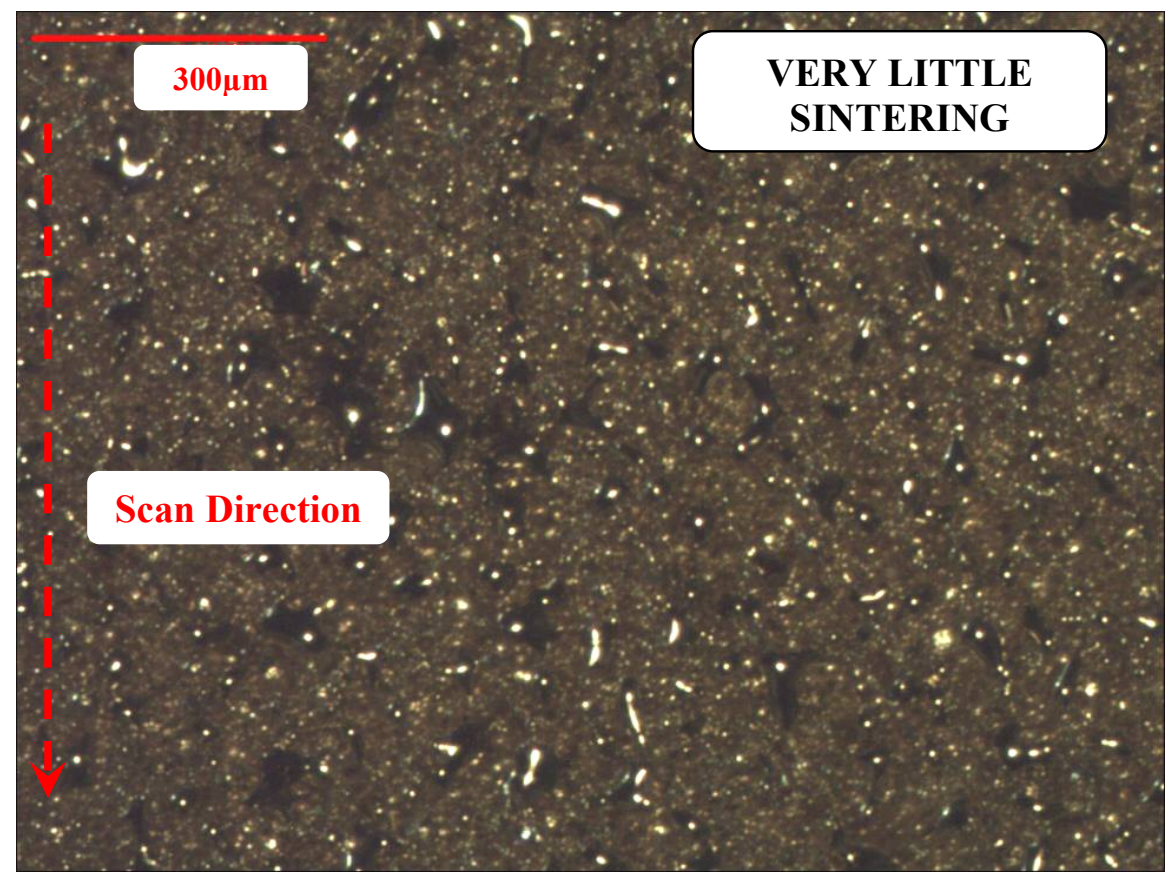

Figure 8-18: Microscope image of single layer scan of gold: very little sintering at 10W laser power and $25 \mathrm{~mm} / \mathrm{s}$ scan speed 


\subsubsection{First Line Balling}

A first line balling effect was also observed for laser power and scan speed combinations of $50 \mathrm{~W}$ and $25 \mathrm{~mm} / \mathrm{s}, 35 \mathrm{~W}$ and $65 \mathrm{~mm} / \mathrm{s}, 50 \mathrm{~W}$ and $65 \mathrm{~mm} / \mathrm{s}, 35 \mathrm{~W}$ and $25 \mathrm{~mm} / \mathrm{s}$ and $45 \mathrm{~W}$ and $100 \mathrm{~mm} / \mathrm{s}$ as shown in Table $8-2$. It was observed that these parameters which produce first line balling had a high laser power and low scan speed combination which ensured enough energy going into the gold powder to cause it to melt and form balling. The large size of the droplets/balling (up to $260 \mu \mathrm{m}$ ) (Figure 8-19) also suggested enough energy and time available (due to high laser power and low scan speed) for the melt pool to split and form balling as explained earlier in section 8.1.1. No such effect was observed for all other parameter setting used in Table 8-2 where these parameters had either a low laser power or a high scan speed.

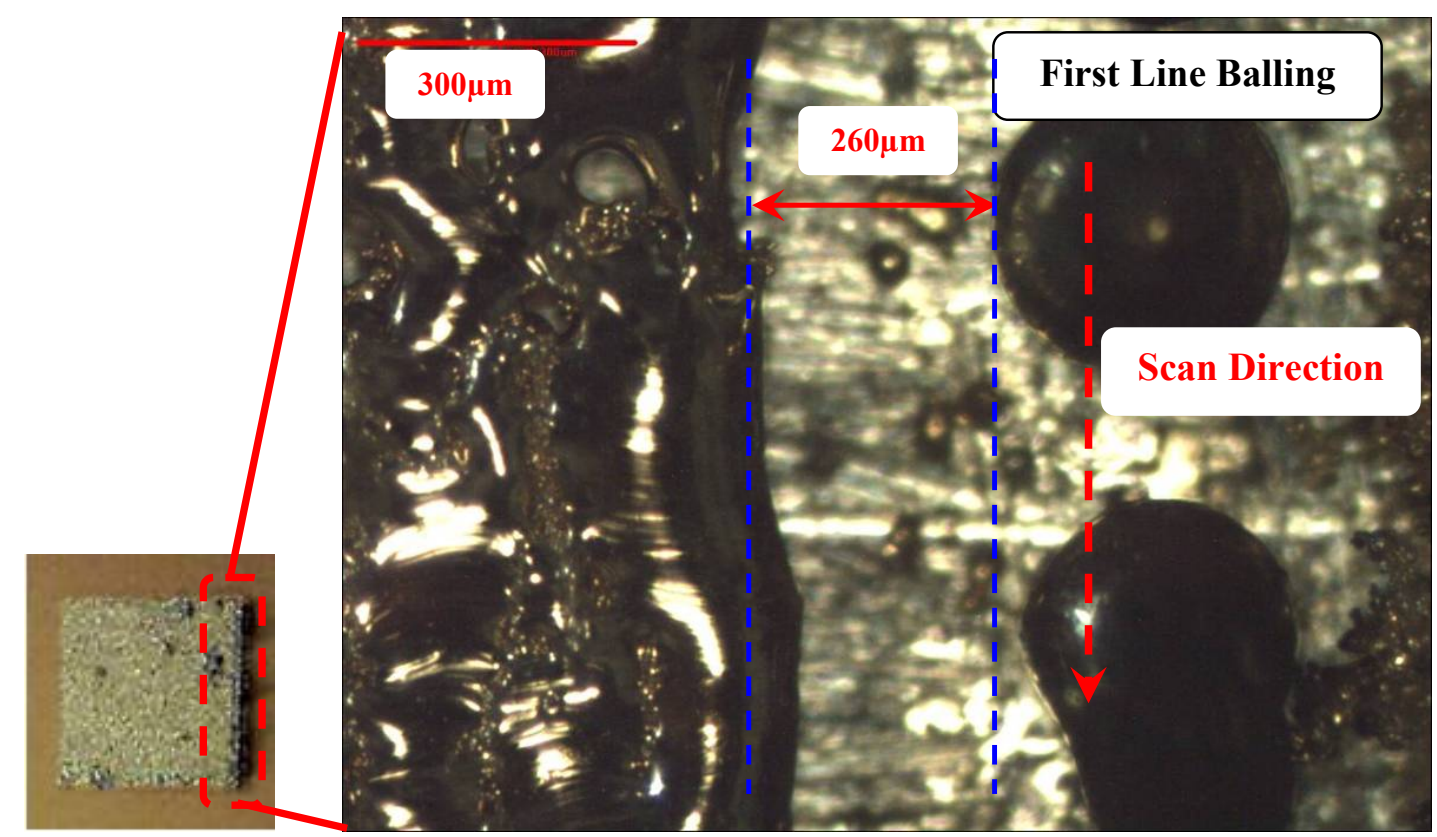

Figure 8-19: Microscope image of first line balling at $50 \mathrm{~W}$ laser power and $65 \mathrm{~mm} / \mathrm{s} \mathrm{scan}$ speed

The possible explanation for no balling for the subsequent scans (scans after the first line balling) could be that when the laser starts processing the first scan on the powder bed, there was powder available on both sides of the laser path which melted and created a larger melt pool (Figure 8-20a). For the following scans there would be almost no powder available on one side which would result in a smaller melt pool, reduced time in the molten state and hence less chance of balling to occur (Rombouts et al, 2006) as illustrated in Figure 8-20b. 

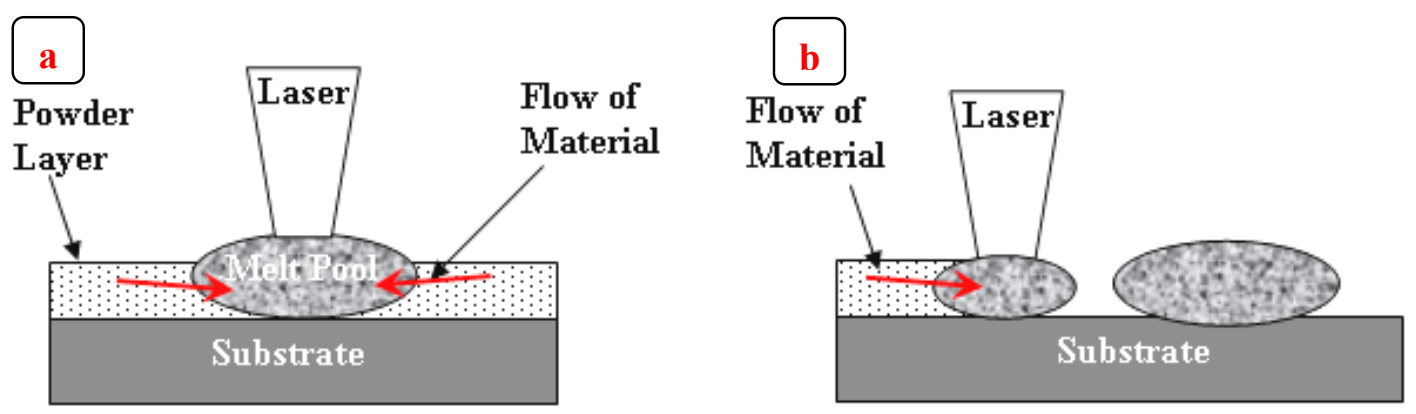

Figure 8-20: (a) laser scanning first scan on the powder layer (b) laser scanning second scan on the powder layer with no powder available on one side

In Figure 8-19, the droplets are approximately $260 \mu \mathrm{m}$ from the edge of the melted layer. As Figure 8-19 was scanned with a hatch distance $80 \mu \mathrm{m}$, there were approximately 3 more laser scans between the droplets and the edge of the melted layer. Based on the position of the droplets, the proposed phenomenon of first line balling could also be referred to as first few lines balling. In this case the balling could have occurred when laser scanned first scan on the powder layer. The subsequent scans, which are $80 \mu \mathrm{m}$ apart, could have re-melted the droplets and the adjacent powder, forming a much larger melt pool and thus resulting in a larger droplet size as seen in this case. Further investigation is required into the formation of melt pool during SLM of gold.

\subsubsection{Adhesion of Gold to the Substrate}

It is important in layer manufacturing that the first layer adheres to the substrate. Although the adhesion of first layer to the substrate does not play any role in the part quality such as density or surface roughness etc. it does prevent the movement/misalignment of the first layer during the deposition of subsequent layers. In all the above experiments, gold did not fuse to the underlying substrate. A number of options were tested in order to eradicate this problem. The first was to use a copper substrate because of its close melting point $\left(1084^{\circ} \mathrm{C}\right)$ with gold $\left(1064^{\circ} \mathrm{C}\right)$ and its alloying ability. A single $100 \mu \mathrm{m}$ thickness layer was spread over the copper substrate and single scans were performed. The parameters used were the same as listed in Table 8-2. However, the single scans obtained in this manner also failed to fuse to the copper substrate. The gold is very reflective compared to other metal powders (explained in detail in Chapter 5) and therefore it does not absorb enough energy initially (as shown in section 2.2.3). In addition to this, once the gold powder starts melting during the laser processing, some of the absorbed energy is utilised in phase change (latent heat of fusion) rather than heat transfer to the 
underlying substrate thus making it difficult to heat the underlying substrate to the desired temperature and induce fusion.

This non-fusion of gold to the substrate was eventually solved by drilling multiple fill holes in the steel substrate as shown in Figure 8-21. These holes were $2 \mathrm{~mm}$ in diameter and $3 \mathrm{~mm}$ deep. When the first powder layer was deposited on the substrate, these holes were filled with the gold powder. When this was deposited the first layer was scanned with the laser, the gold in these holes also melted and kept the first layer in its position during the subsequent layer deposition process.

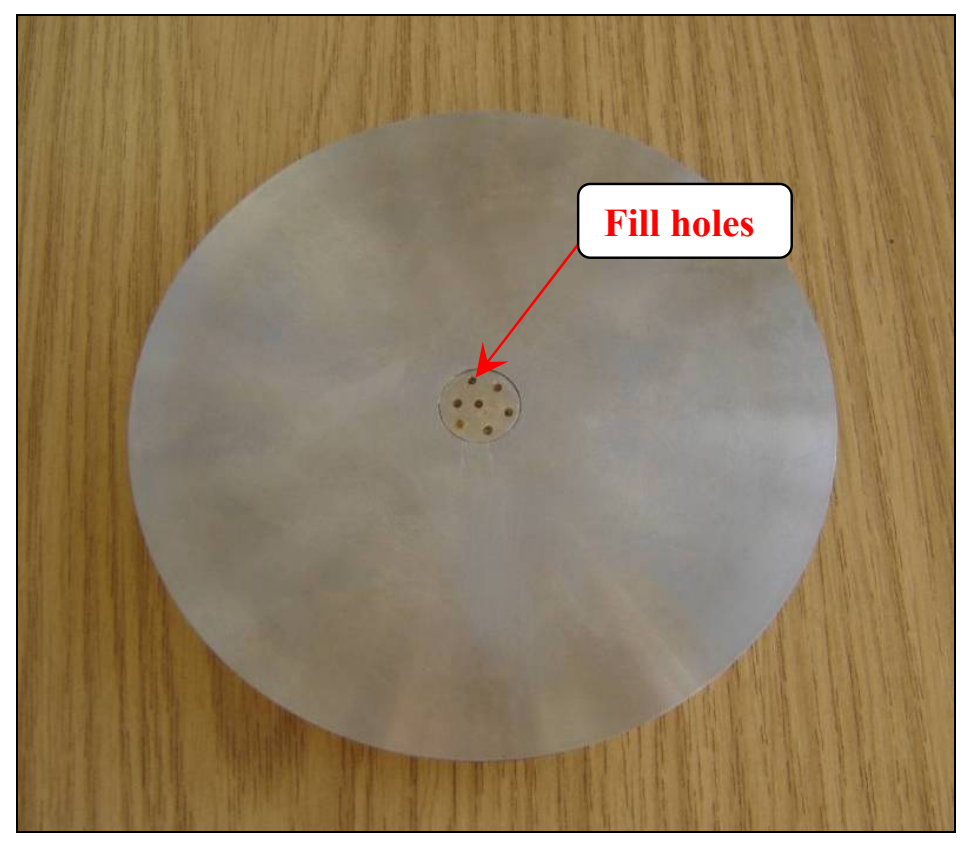

Figure 8-21: Smaller build platform with fill holes

\subsection{Manufacturing of Gold Cubes}

Multiple layer parts were created from gold using the new powder deposition system and build platform (explained in detail in Chapter 7). From the results obtained from the single line and single layer manufacturing, the good melting region was expected to be suitable for multiple layer part manufacturing. A maximum laser power of $50 \mathrm{~W}$ and a scan speed of $65 \mathrm{~mm} / \mathrm{s}$ was used for further experiments (as selected in section 8.3). A maximum laser power of $50 \mathrm{~W}$ was selected because it ensured a maximum energy input for the highly reflective gold powder (approximately 85\% reflective). A scan speed of $65 \mathrm{~mm} / \mathrm{s}$ was selected from the very small good melting region in Figure 8-2. A layer thickness of $100 \mu \mathrm{m}$ was used for the gold cube manufacturing as also used for single scan 
and single layer manufacturing in section 8.1 and 8.3. The effect of layer thickness on the porosity of the gold cubes is explained in detail in Chapter 9.

A few samples of gold cubes, $3 \times 3 \times 3 \mathrm{~mm}$ in size, were first produced to check the working of the new deposition system and build platform. This size of gold cubes was selected in view of the smaller build platform $(15 \mathrm{~mm}$ in diameter) and the small amount of material available. The gold powder was first sieved before it was put in the smaller hopper (explained in detail in section 7.5.2). The rotation of the wiper was limited to $50^{\circ}$ (15 to $65^{\circ}$ ), which ensured the powder deposition only over the smaller build platform thus limiting the spreading of powder inside the chamber. The bed temperature was kept at $100^{\circ} \mathrm{C}$ which was limited by the smaller build platform (explained in section 7.5.7) and also used for the single lines and layer manufacturing explained earlier in this Chapter. Figure 8-22 shows an SEM image of a gold cube produced with a laser power of 50W and scan speed of $65 \mathrm{~mm} / \mathrm{s}$. The bottom side had a much greater roughness due to the supports attached to this surface. The gold cubes were successfully manufactured using the new build platform and the smaller deposition system. A detailed analysis of the internal porosity and mechanical properties is presented in Chapter 9.

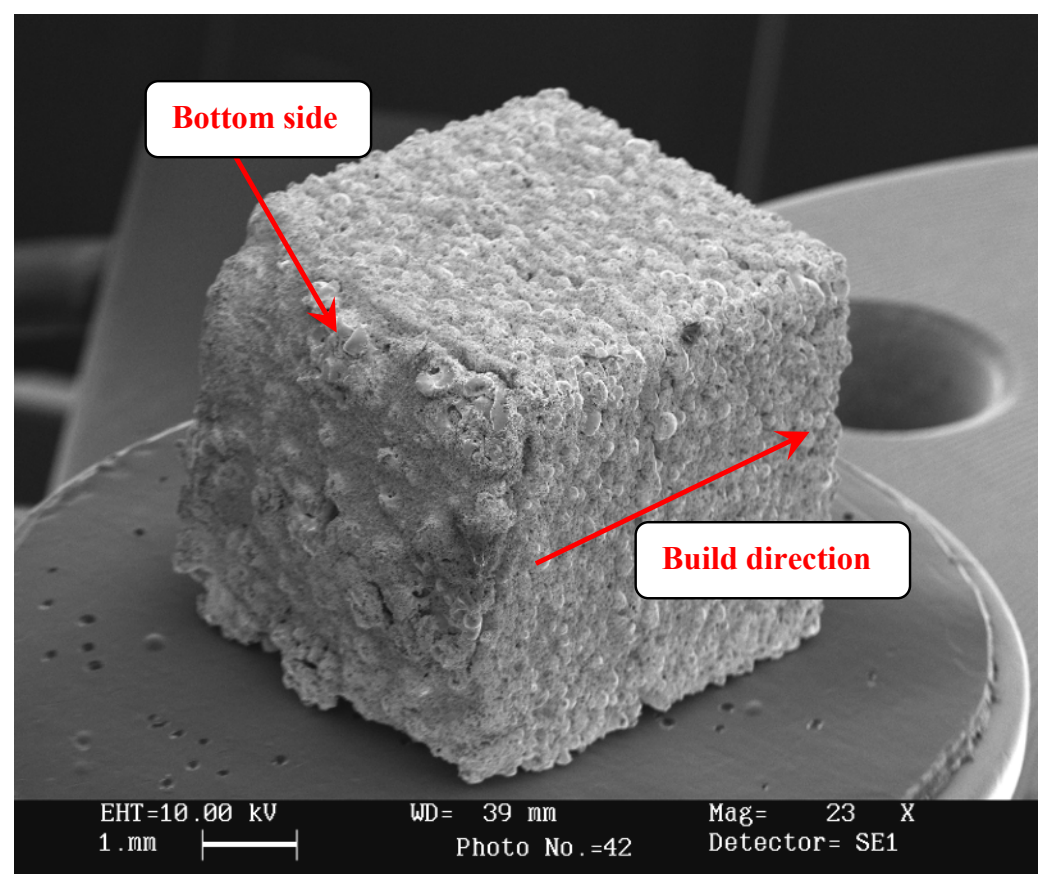

Figure 8-22: Scanning Electron Microscope (SEM) image of gold cube

\subsection{Summary}

A number of experiments were performed to identify a suitable processing window for gold powder. A very narrow window of good processing was successfully identified 
within a number of other processing regions. The balling effect was also observed during the processing of gold powder. It was noted that the size of droplets in the balling region increased with decreasing scan speed and increasing laser power. It was also observed that balling at high laser power and low scan speeds had very little or no powder available on either one or both sides of the laser scan path. The gold powder was found to melt very little below a laser power of 15 to $25 \mathrm{~W}$ for a full scan speed range of 10 to $500 \mathrm{~mm} / \mathrm{s}$. Various selective experiments were also performed for single layers where a first line balling effect was observed.

All the experiments were performed on the steel substrate but gold did not fuse to the substrate. This problem was resolved by making fill holes in the substrate. The gold cubes were successfully manufactured with the new powder deposition system and the smaller build platform using the suitable processing parameters from the good melting region of the processing window.

The next chapter analyses gold cubes for their internal porosity and mechanical properties etc. 


\section{Chapter 9}

\section{ANALYSIS OF GOLD CUBES}

\subsection{Porosity Measurement of Gold Cubes}

Gold cubes $4 \times 4 \times 4 \mathrm{~mm}$ in size were produced using the new powder deposition system and build platform for analysis. A size larger than $4 \times 4 \times 4 \mathrm{~mm}$ could be easily produced but this size was found to be suitable for porosity measurements. With this size, three samples were produced in a single build with the new build platform and deposition system, whereas a size larger than this would have increased the material required as well as wastage with increasing number of builds. The small quantity of gold powder available for this research work was a key limiting factor.

There are two different ways the density of the samples could be obtained i.e. the Archimedes principle and the cross-sectioning technique. The Archimedes principle is a non-destructive testing technique in which the sample is weighed in air and water and these values are used to calculate the density of the parts. The advantage of this technique is the non-destructive nature of the procedure where the part is recovered after measurements while the disadvantage is that the part cannot be examined from inside. The crosssectioning technique is a destructive testing technique in which the samples are crosssectioned (by a diamond cutter or Wire Electric Discharge Machine (WEDM) etc.) or ground (silicon carbide grind wheels etc) to different sections and then examined through an optical microscope for internal porosity. The advantage of this process is that the inside of the part can be examined for porosity, layer delamination and mechanical properties such as hardness and modulus etc. The disadvantage of this process is that a certain amount of material is always lost during the cutting or grinding process and the sample is destroyed.

Keeping in view the advantages and disadvantages of both procedures, the destructive testing technique was adopted in which gold cubes were cross-sectioned and examined for porosity using optical methods. This technique gave a better understanding of internal part porosity at different locations and also the layer delamination problem. 


\subsubsection{Sample Preparation}

The gold cubes were first placed in sample holders and then placed in circular sample cups. Epo Thin epoxy resin and epoxy hardner were mixed in a ratio of 10 to 3.9 grams by weight respectively for each sample. This mixture was stirred for a few minutes and poured into the cups containing the gold cubes. These cups were placed in a low pressure container for 5 minutes to force the resin to enter into the small pores. The samples were cured and hardened at room temperature for 18 to 20 hours.

After curing, the samples were ground with different size silicon carbide papers. Because of the soft nature of the 24 carat gold samples, special care was taken during the grinding process in order to avoid any particles or chips embedding in another location on the sample which could give errors in measuring density of the parts. All the samples were ground with 200, 400, 600 and 800 grit papers respectively before a final finish with 6 and $1 \mu \mathrm{m}$ grinding wheels. The grinding of these samples however left some scratches on the samples which are visible in the figures.

\subsubsection{Results and Discussion}

Initially a layer thickness of $100 \mu \mathrm{m}$ was used to produce gold cubes. The samples were prepared as explained in the previous section. An optical microscope was used to take images of the cross section of these samples. The image analysis was performed with the free image analysis tool UTHSCSA, developed by The University of Texas Health Science Center in San Antonio (UTHSCSA, 2002). The UTHSCSA Image Tool (IT) can acquire, display, edit, analyze, process, compress, save and print grey scale and coloured images. Image analysis functions include dimensional (distance, angle, perimeter, area) and grey scale measurements (area measurements based on grey scale level) which were used in this case. All the gold cube samples were analyzed at three different cross sections and the average porosity was obtained for each sample.

As gold was already known to be highly reflective in nature (from Chapter 5), high power was required to deliver enough energy to melt the gold powder. The maximum energy delivery to the gold powder was achieved by combining a high laser power with a low scan speed, the values lying towards the upper left corner of Figure 8-2. This was confirmed by identifying the good melting region which occurred towards the upper left corner of Figure 8-2. Therefore, the region between the laser power of 25 to $50 \mathrm{~W}$ and scan speed of 10 to $130 \mathrm{~mm} / \mathrm{s}$ was considered to be the region of interest for further experiments. 
Due to the small quantity of gold powder available, only a limited number of experiments could be performed for gold cube manufacturing, so from this region of interest, a maximum laser power of $50 \mathrm{~W}$ was selected to ensure maximum energy delivery to the highly reflective gold powder, whereas a scan speed of 25, 65, 100 and $130 \mathrm{~mm} / \mathrm{s}$ was selected from the balling, unstable melt and good melting regions. A hatch distance of $80 \mu \mathrm{m}$ was selected as explained in detail in section 8.3.

The processing parameters for gold cubes are summarized in Table 9-1. Three gold cubes were prepared for each set of parameters and these were analyzed for internal porosity. Each of the gold cubes was checked at three sections for their internal porosity. The change in density with scan speed was investigated for these samples.

\begin{tabular}{|c|c|c|c|c|}
\hline $\begin{array}{c}\text { Laser Power } \\
(\mathbf{W})\end{array}$ & $\begin{array}{c}\text { Scan speed } \\
(\mathrm{mm} / \mathrm{sec})\end{array}$ & $\begin{array}{c}\text { Hatch Distance } \\
(\boldsymbol{\mu m})\end{array}$ & $\begin{array}{c}\text { Layer } \\
\text { Thickness }(\mu \mathrm{m})\end{array}$ & $\begin{array}{c}\text { Bed } \\
\text { temperature } \\
\left({ }^{\circ} \mathbf{C}\right)\end{array}$ \\
\hline 50 & 25 & 80 & 100 & 100 \\
\hline 50 & 65 & 80 & 100 & 100 \\
\hline 50 & 100 & 80 & 100 & 100 \\
\hline 50 & 130 & 80 & 100 & 100 \\
\hline
\end{tabular}

Table 9-1: $\quad$ Parameters and their values for gold cubes manufacturing

Figure 9-1 shows the variation in the porosity with changes in scan speeds for these gold cubes. From this figure, it can be seen that at a scan speed of $65 \mathrm{~mm} / \mathrm{s}$ there occurred the lowest average porosity of $14.4 \%$, whereas the highest was $18.2 \%$ for a scan speed of $130 \mathrm{~mm} / \mathrm{s}$.

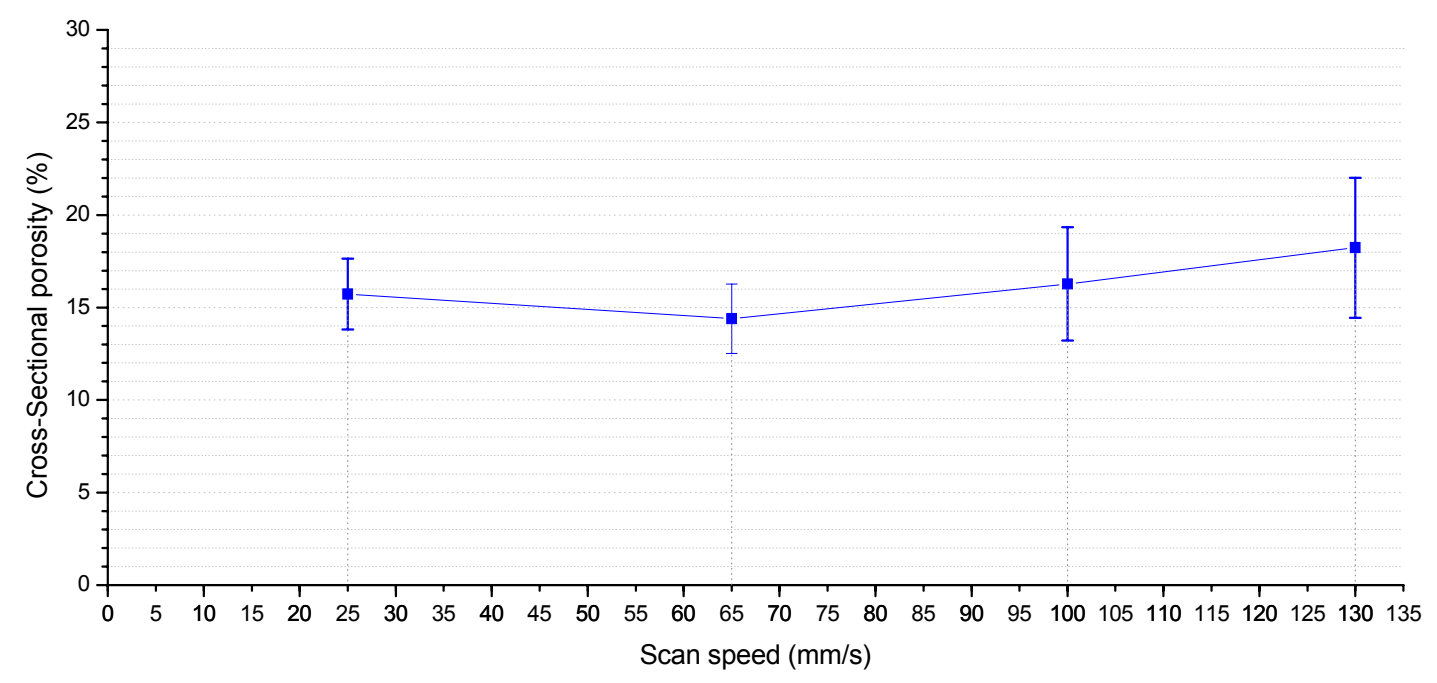

Figure 9-1: Cross-sectional porosity variation with scan speed in gold cubes 
The set of samples prepared at $50 \mathrm{~W}$ and $25 \mathrm{~mm} / \mathrm{s}$ had an irregular and broken layered structure. The average porosity of these samples was found to be $15.7 \%$. These parameters were taken from the balling region (Figure 8-2) and as explained earlier in section 8.3, a poor layer quality was responsible for high porosity in these parts. A cross section image of one of the samples prepared at $50 \mathrm{w}$ and $25 \mathrm{~mm} / \mathrm{s}$ is shown in Figure $9-2$.

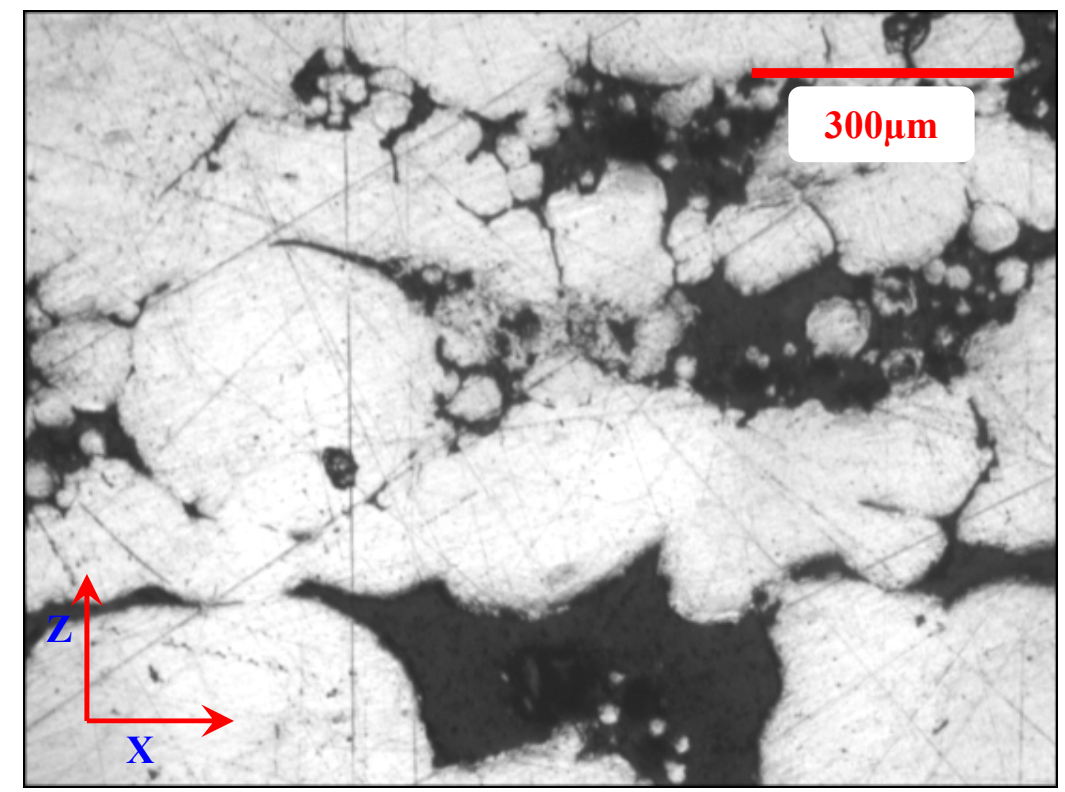

Figure 9-2: Cross section image of sample produced with a scan speed of $25 \mathrm{~mm} / \mathrm{s}$ and laser power of $50 \mathrm{~W}(17.7 \%$ porosity $)$

The samples produced with processing parameters of $50 \mathrm{~W}$ and $65 \mathrm{~mm} / \mathrm{s}$ showed the best results in terms of part porosity (average porosity of $14.4 \%$ ). Also in these parts, most of the porosity was observed to be inter-layer porosity rather than intra-layer porosity. The high porosity between the layers suggests less heat was not transferred to the underneath layers, thus resulting in weak bonding between consecutive layers. The high reflectivity of gold powder (approximately 85\%) could be the possible reason for the low energy transferred to the underlying layers. This reflectivity also increases as the gold powder melts and forms a solid layer during the laser processing, thus further reducing the amount of energy absorbed. Due to this reason there was very little melting and most of the porosity was observed in the region between the layers. Figure 9-3 shows an image of one of the samples produced with these parameter settings. 


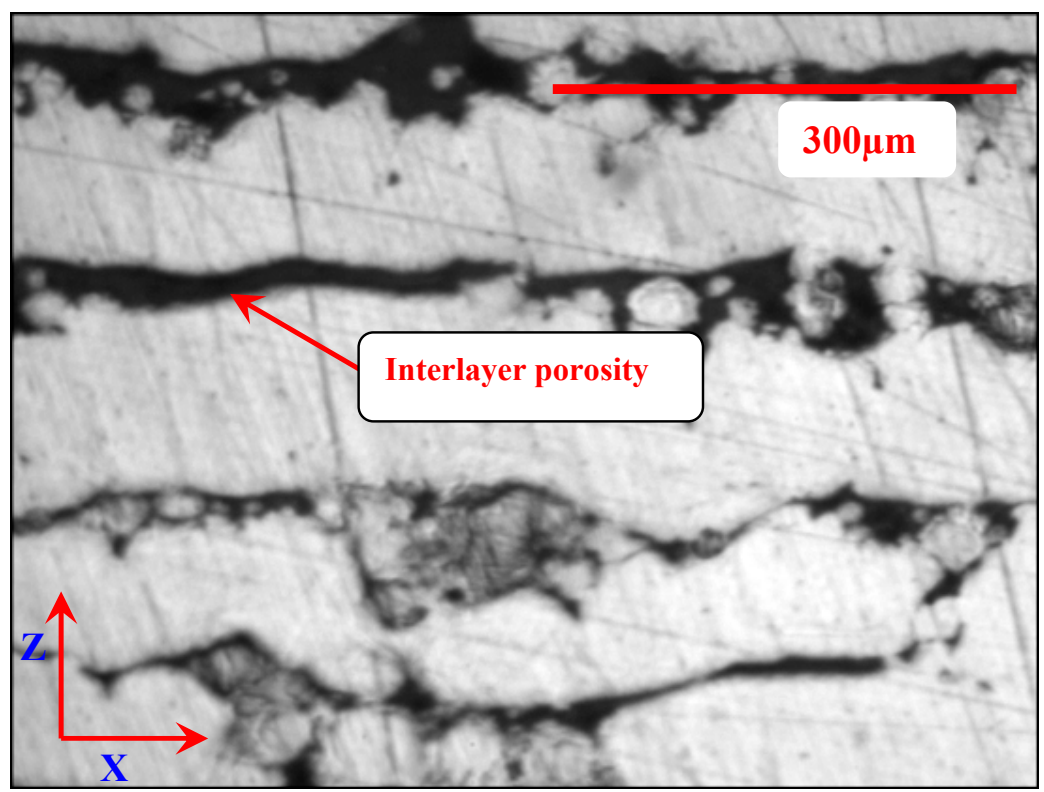

Figure 9-3: Cross section image of sample produced with a scan speed of $65 \mathrm{~mm} / \mathrm{s}$ and laser power of $50 \mathrm{~W}(12.5 \%$ porosity $)$

The other two parameter settings i.e. 100 and $130 \mathrm{~mm} / \mathrm{s}$ yielded samples with higher porosity. The average porosity of samples produced at $100 \mathrm{~mm} / \mathrm{s}$ and $130 \mathrm{~mm} / \mathrm{s}$ was found to be $16.3 \%$ and $18.2 \%$ respectively. The possible reason for high porosity at these settings could be that these parameters were selected from unstable melt and balling region (similar to $25 \mathrm{~mm} / \mathrm{s}$ samples) where both of these regions showed poor layer quality (Section 8.3). Figure 9-4 and 9-5 show images of parts from each of these parameters.

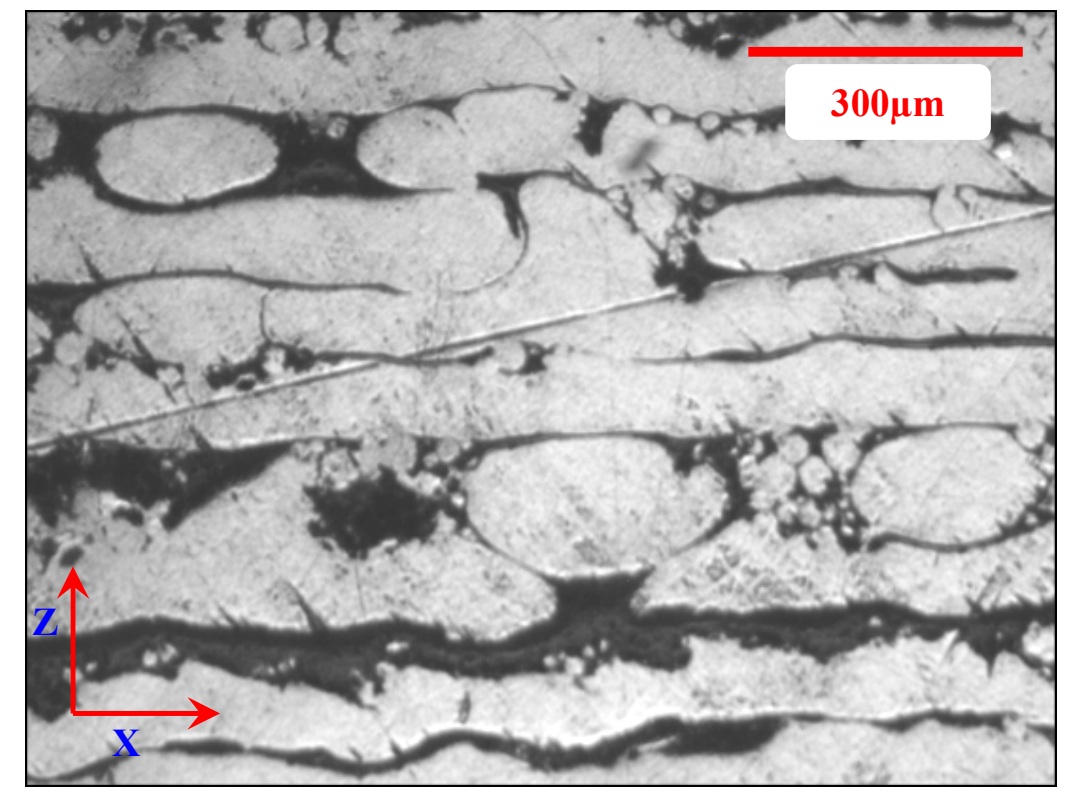

Figure 9-4: Cross section image of the sample produced with a scan speed of $100 \mathrm{~mm} / \mathrm{s}$ and laser power of $50 \mathrm{~W}(13.2 \%$ porosity) 


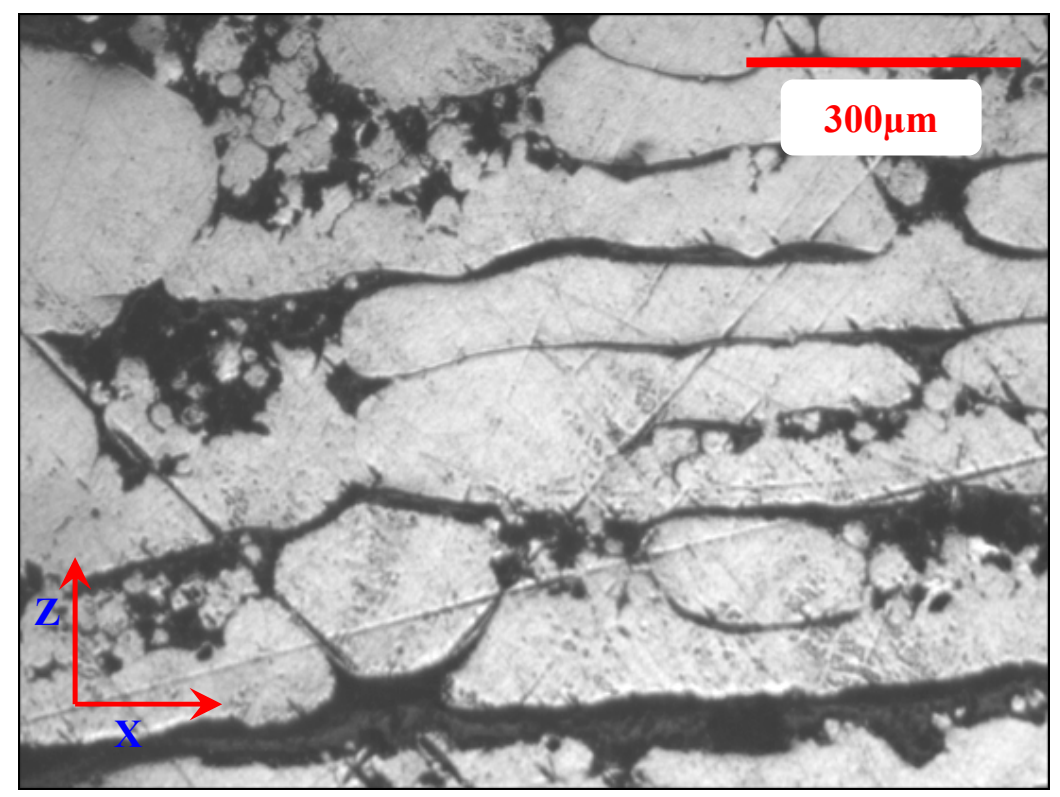

Figure 9-5: Cross section image of the sample produced with a scan speed of $130 \mathrm{~mm} / \mathrm{s}$ and laser power of $50 \mathrm{~W}$ (14.4\% porosity)

In all of the cross-section images, the boundary scan area and contour fill scan area had similar levels of porosity.

\subsection{Improvement in the Density of Gold Cubes}

In the previous section, the minimum porosity $(12.5 \%)$ was obtained for a scan speed of $65 \mathrm{~mm} / \mathrm{s}$ (Figure 9-3). The major part of this porosity was found to be the interlayer porosity which could be attributed to the low amount of energy transferred to the region between two layers. According to equation (9.1), four factors influence the total amount of energy supplied to the gold powder layer. These parameters are the laser power $(\mathrm{P})$, scan speed $\left(v_{\text {scan }}\right)$, hatch distance $\left(\mathrm{d}_{\mathrm{h}}\right)$ and layer thickness $\left(\mathrm{T}_{\text {layer }}\right)$. Apart from these parameters, the powder bed preheating temperature could also be used to reduce the energy required to melt the gold powder. A higher bed temperature is already known to have some effect on reduction of the energy required to melt a material (Van Elsen, 2007) and in this case, the powder bed preheating could reduce the high energy requirement for a highly reflective material like gold. The four parameters (as shown in equation (9.1)) could be adjusted so as to increase the energy delivered to the powder. This section explains in detail the effect of each of the above mentioned parameters on the density of the final part.

$$
E_{\text {density }}=\frac{P_{\text {laser }}}{v_{\text {scan }} \cdot d_{h} \cdot T_{\text {layer }}}
$$




\subsubsection{Laser Power}

As the SLM 100 system uses a 50W laser source and the parts were built using a maximum power of $50 \mathrm{~W}$, the laser power could not be increased.

\subsubsection{Scan speed}

As observed previously, the best porosity results were obtained at a scan speed of $65 \mathrm{~mm} / \mathrm{s}$ (Figure 9-3). Therefore this scan speed was not changed during further experimentation.

\subsubsection{Bed Temperature}

The bed temperature of $100^{\circ} \mathrm{C}$ was used for the gold cubes produced in this research and also for the single scans and single layers experiments. The powder bed preheating temperature was limited by the new build platform as explained in section 7.5.7. It was observed that at room temperature the layers curled due to the high thermal gradient and shrinkage which forced the process to be stopped. Figure 9-6 shows the damaged layers due to shrinkage and thermal stresses at lower bed temperature (room temperature) compared to a good uniform layer with a higher bed temperature of $100^{\circ} \mathrm{C}$.
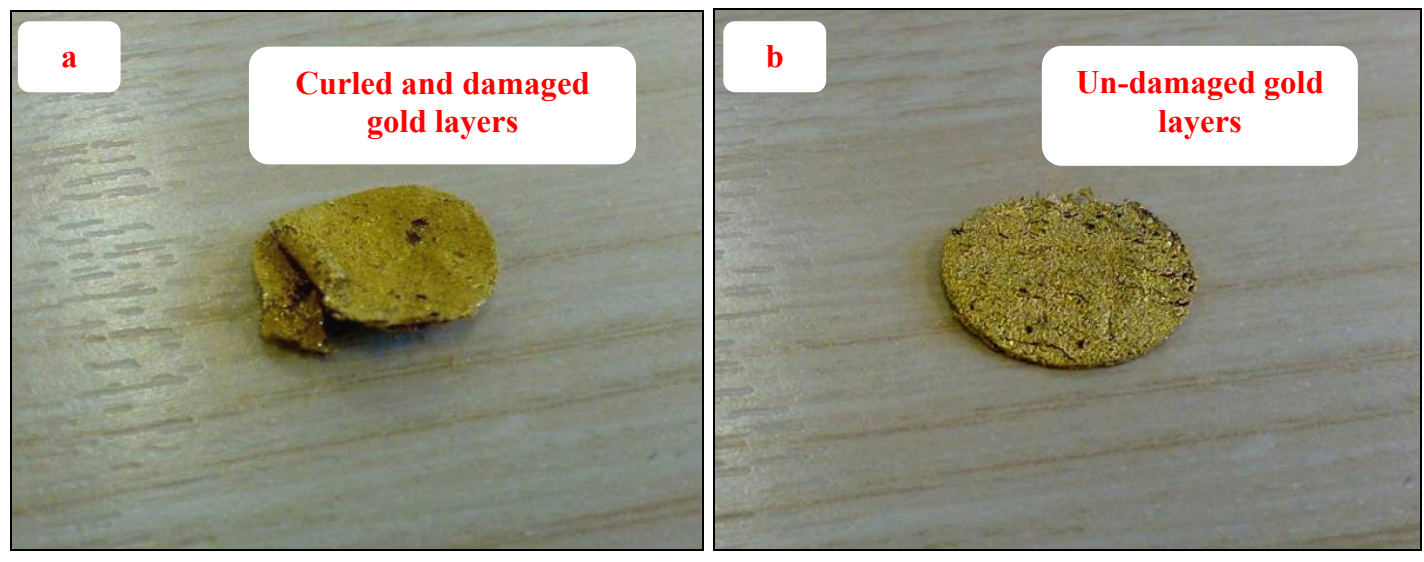

Figure 9-6: Multiple layer gold discs (a) bed temperature $28^{\circ} \mathrm{C}$ (room temperature) (b) bed temperature $100^{\circ} \mathrm{C}$

\subsubsection{Layer Thickness}

Decreasing the layer thickness could increase the energy density per unit volume from equation (9.1). To check the effect of layer thickness, gold cubes with layer thickness of 75 and $50 \mu \mathrm{m}$ were also produced and analyzed for internal porosity. These gold cubes were produced with a laser power of $50 \mathrm{~W}$, scan speed of $65 \mathrm{~mm} / \mathrm{s}$ and hatch distance of 
$80 \mu \mathrm{m}$. Three samples were prepared for each parameter setting. The change in the layer thickness from 100 to $75 \mu \mathrm{m}$ had a negligible effect whereas with the $50 \mu \mathrm{m}$ layer thickness the average porosity was reduced from $14.4 \%$ to $10.4 \%$ (Figure 9-7). A reduction in porosity with decrease in layer thickness was observed to be due to the reduction in the gap between the two layers where porosity was found as shown in Figure 9-8. Although there was still interlayer porosity, the minimum porosity obtained with a layer thickness of $50 \mu \mathrm{m}$ was $7.7 \%$ as shown in Figure 9-8.

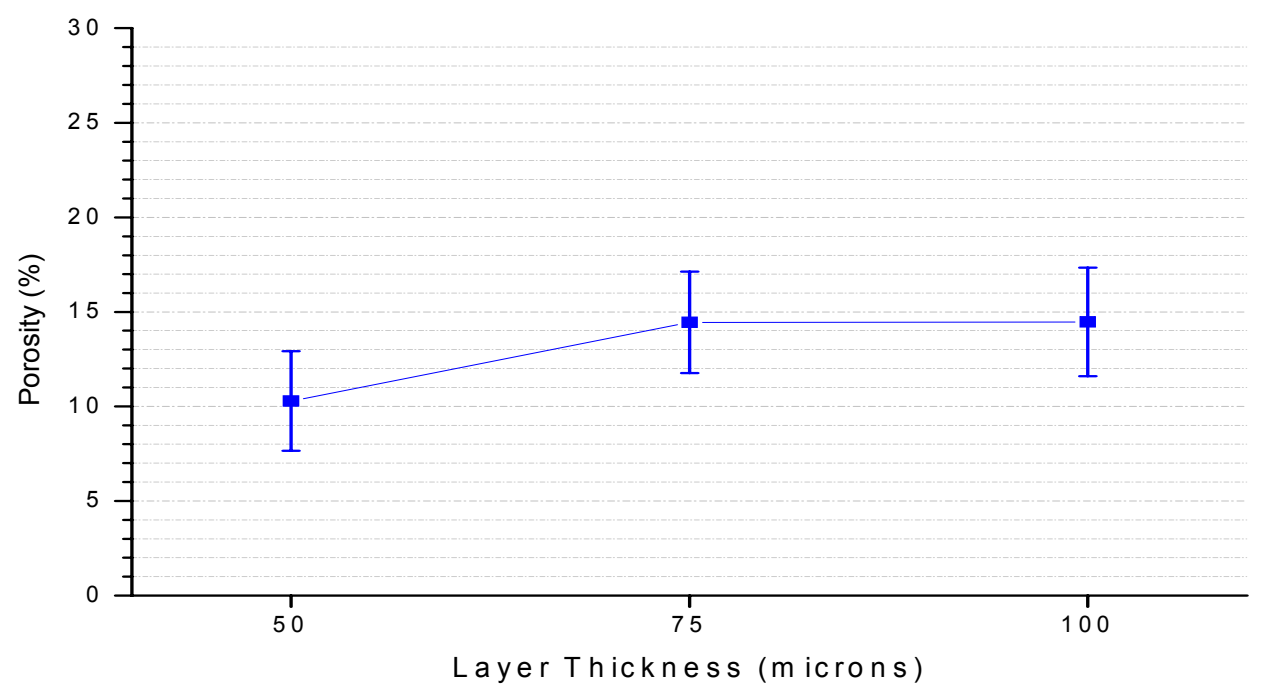

Figure 9-7: Porosity variation with layer thickness

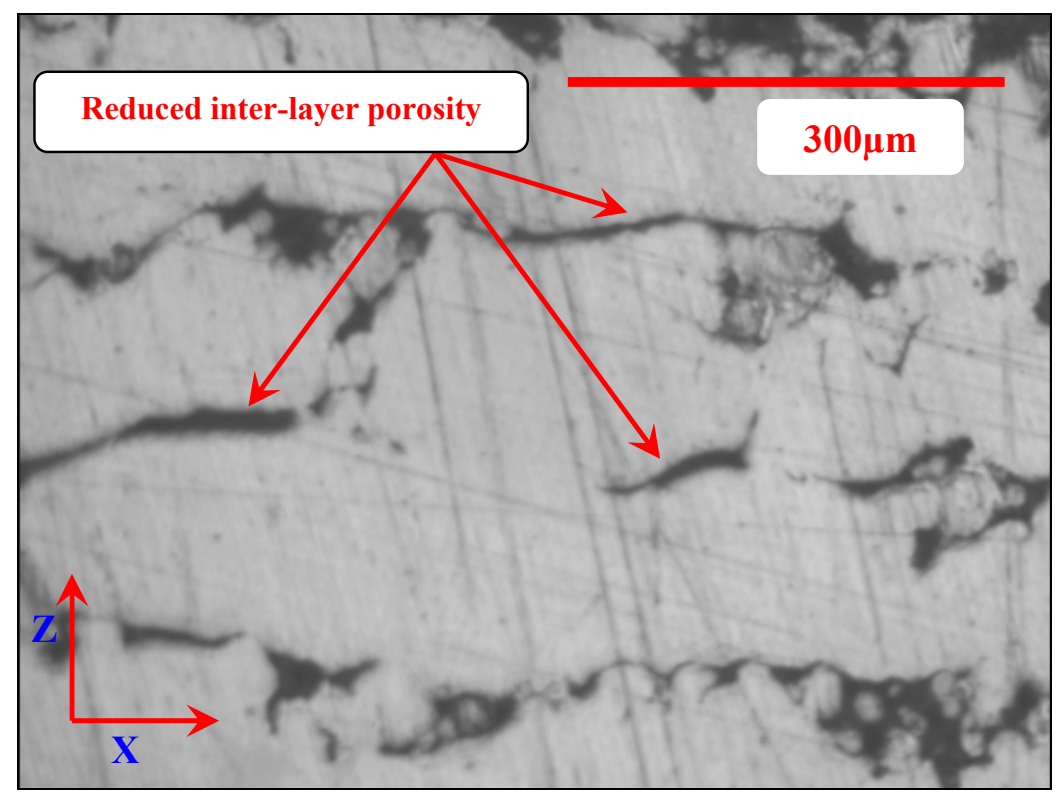

Figure 9-8: Cross section image of the sample produced with a scan speed of $65 \mathrm{~mm} / \mathrm{s}$, laser power of $50 \mathrm{~W}$ and layer thickness of $50 \mu \mathrm{m}(7.7 \%$ porosity $)$ 


\subsubsection{Hatch Distance}

The gold cubes were also checked for the effect of hatch distance on the internal porosity. A laser power of $50 \mathrm{~W}$, scan speed of $65 \mathrm{~mm} / \mathrm{s}$ and layer thickness of $50 \mu \mathrm{m}$ were used to produce these gold cubes. Three samples were produced for each parameter setting. The hatch distance was changed from 80 to 60 and $40 \mu \mathrm{m}$. It was observed that the hatch distance had a negligible effect on porosity of the gold cubes as shown in Figure 9-9. Although the reduction in hatch distance increased the energy density, it was still not enough to have a significant influence on the depth to which the heat energy was transferred. This left the area between the two layers un-melted; hence the porosity remained almost the same. The non-adhesion of layers could also be influenced by the formation of oxide films (discussed in section 2.3.3.3) or other contaminants during the SLM processing. This could be responsible for the layer delamination problems in the gold cubes. Further investigation is required into the presence of oxides in the sintered gold cubes.

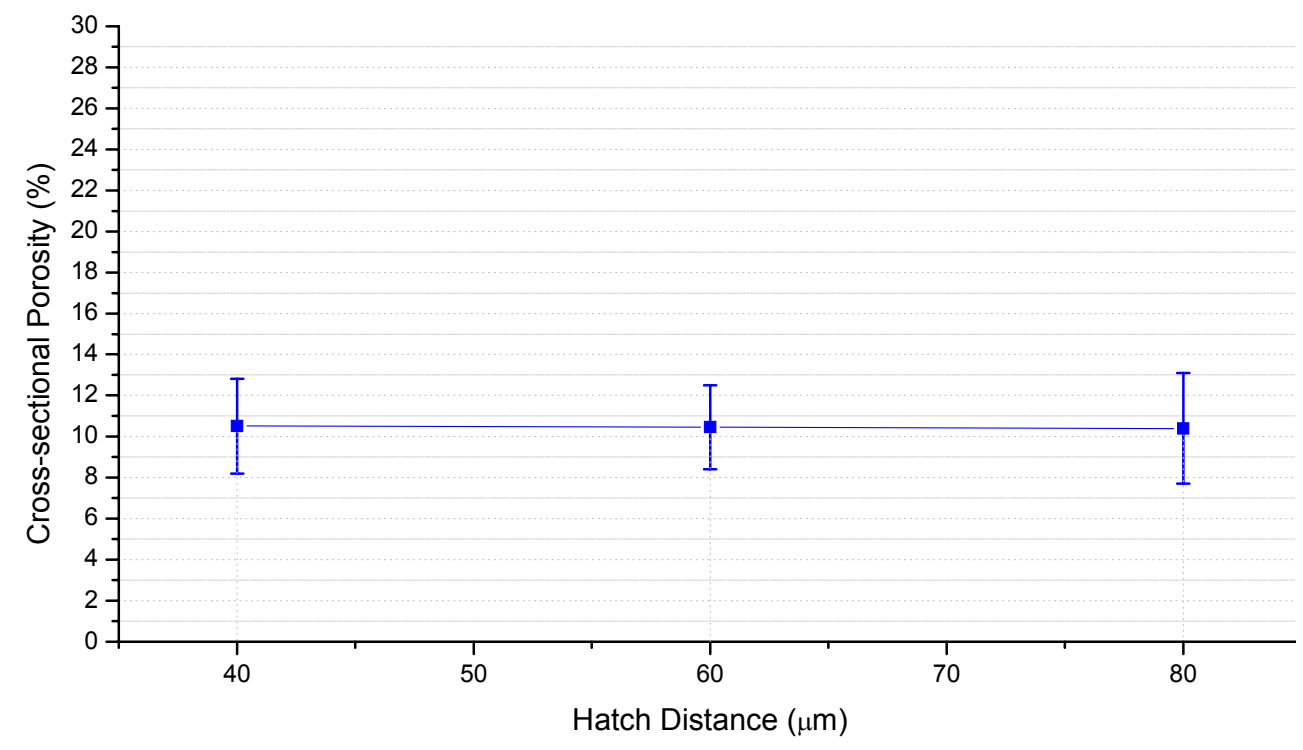

Figure 9-9: Porosity variation with hatch distance

\subsection{Intra Layer Porosity}

The porosity was checked in the previous sections to analyse the quality of the gold cubes. The inter layer porosity constituted the major portion of this porosity. In this section, the intra layer porosity was measured to check the layer quality at different scan speeds (Figure 9-10). The gold cubes produced with a scan speed of $65 \mathrm{~mm} / \mathrm{s}$ had relatively low intra layer porosity compared to a scan speed of 25 and $100 \mathrm{~mm} / \mathrm{s}$. Figure $9-11$ shows intra layer porosity of the sample produced with $65 \mathrm{~mm} / \mathrm{s}$ scan speed. 


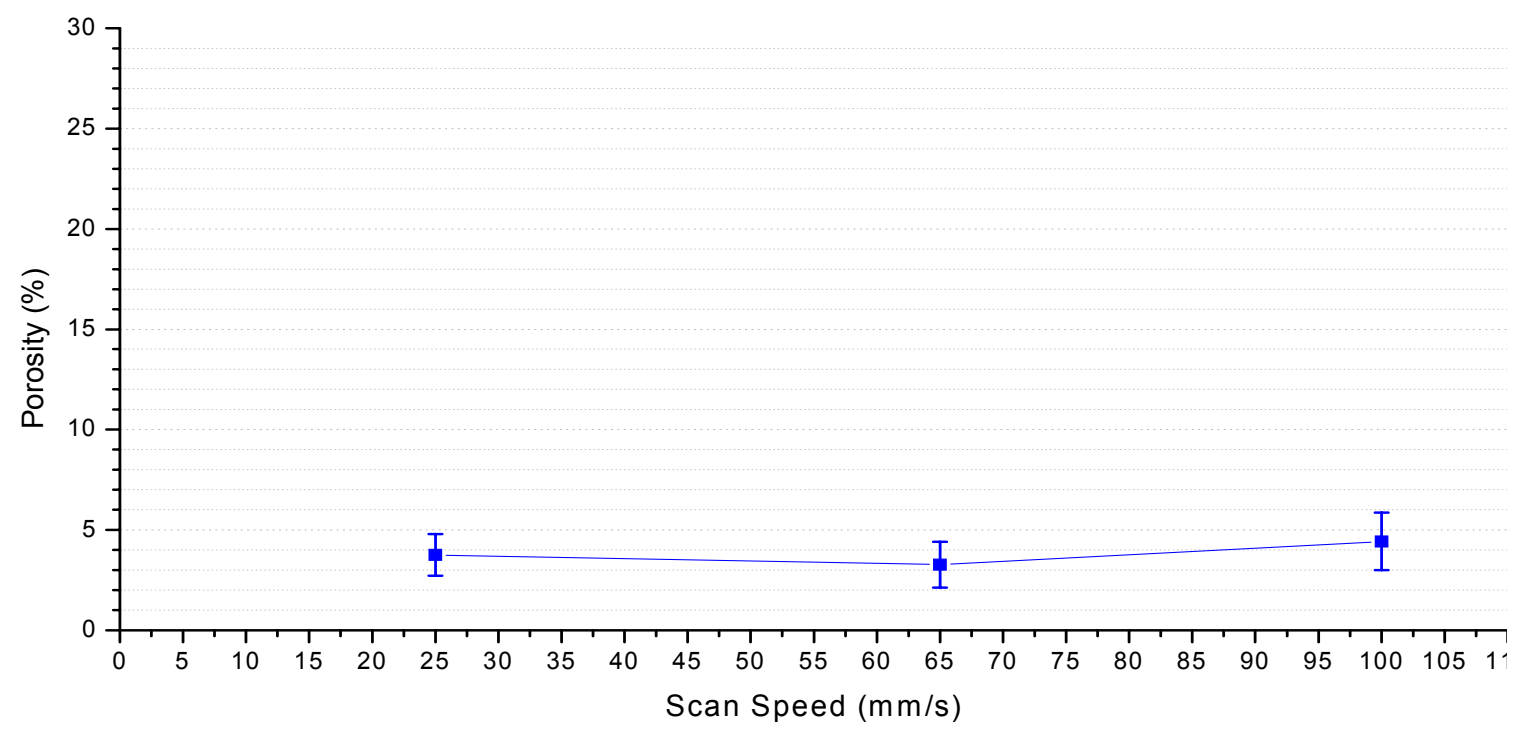

Figure 9-10: Intra layer porosity variation

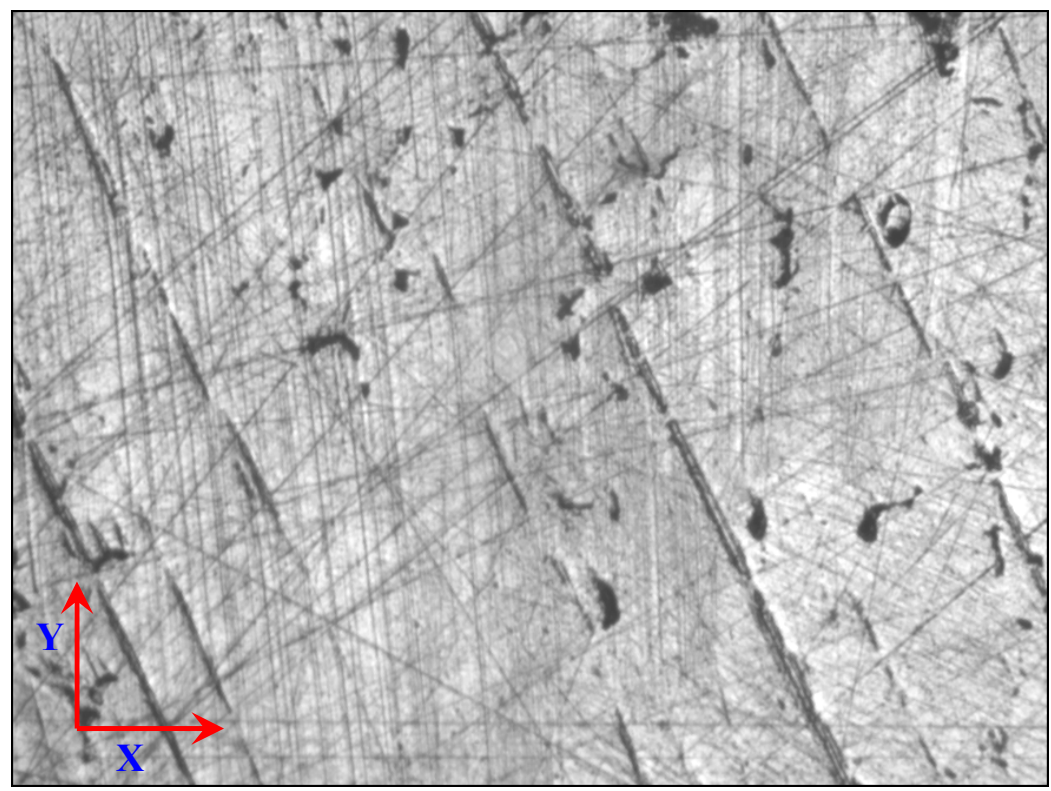

Figure 9-11: Cross section image of the sample produced with a scan speed of $65 \mathrm{~mm} / \mathrm{s}$, laser power of $50 \mathrm{~W}$ and layer thickness of $50 \mu \mathrm{m}(4.4 \%$ porosity $)$

\subsection{Optimum Processing Parameters}

Based on the discussion in Chapter 8 and the porosity measurements in this chapter, the processing parameters summarized in Table 9-2 were selected as the optimum processing parameters. 


\begin{tabular}{|c|c|}
\hline Parameters & Value \\
\hline Laser power & $50 \mathrm{~W}$ \\
\hline Scan speed & $65 \mathrm{~mm} / \mathrm{s}$ \\
\hline Bed temperature & $100^{\circ} \mathrm{C}$ \\
\hline Layer thickness & $50 \mu \mathrm{m}$ \\
\hline Hatch distance & $80 \mu \mathrm{m}$ \\
\hline
\end{tabular}

Table 9-2: $\quad$ Processing parameters

The laser power of 50W was the maximum available laser power in the SLM 100 system. The scan speed of $65 \mathrm{~mm} / \mathrm{s}$ was selected from the good melting region (Figure 8-2) and the gold cubes were found to have minimum porosity at this scan speed (section 9.1.2). the powder bed temperature was limited by the small build platform to a maximum of $100^{\circ} \mathrm{C}$ and it was observed in section 9.2 .3 that a bed temperature of $100^{\circ} \mathrm{C}$ was helpful in reducing the thermal shrinkage in the material. A reduction in layer thickness from $100 \mu \mathrm{m}$ to $50 \mu \mathrm{m}$ was observed to reduce porosity in gold cubes. The hatch distance had a negligible effect on the porosity in gold cubes and therefore it was not changed. The minimum porosity obtained for gold cubes using these optimum parameters was $7.7 \%$ as shown in Figure 9-8.

\subsection{Temperature Measurements in Gold Powder Bed}

Powder preheating is known to reduce the energy required to melt a material (Van Elsen, 2007) and the new build platform used for manufacturing gold cubes could only be preheated to $100^{\circ} \mathrm{C}$. The heat transfer in the gold powder was also checked by placing a thermocouple in the gold powder bed. The main aim of this study was to check whether a pre-scan with a low laser power would heat the gold powder but not melt it and if this could be used as a preheating technique.

A k-type thermocouple was used which could measure temperature up to $1400^{\circ} \mathrm{C}$. The thermocouple was placed $700 \mu \mathrm{m}$ from the top surface of the gold powder in a circular cylinder (Ø15mm x 4mm deep) as shown in Figure 9-12. 


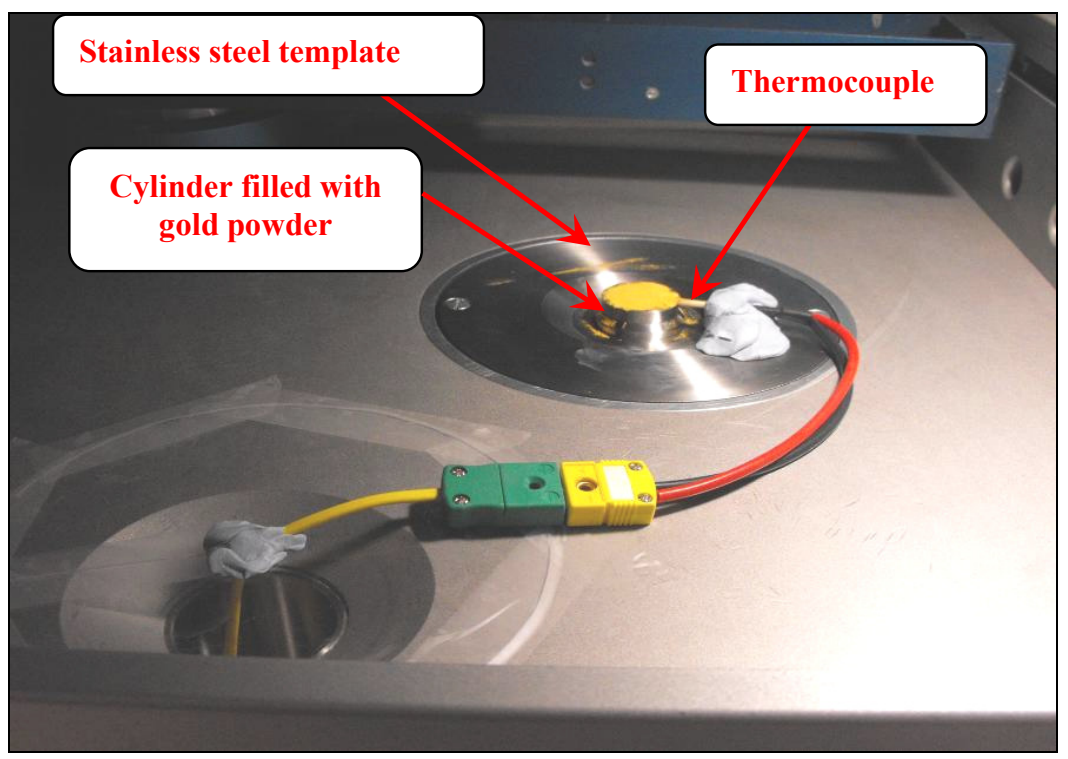

Figure 9-12: Thermocouple placed in the gold powder bed

It was not possible to place the thermocouple any closer to the top surface as there were no means of holding the thermocouple in a horizontal position during the powder scrapping. At this distance, the gold powder was easily spread on top of the thermocouple and the excess powder scraped off. The laser power was varied from 5 to $50 \mathrm{~W}$ with a constant increment of $5 \mathrm{~W}$. Three experiments were performed for each laser power setting and a fresh gold sample was used for each experiment. In order to insulate the thermocouple from the rest of the machine (to prevent short circuiting/ grounding of the thermocouple with the machine), a thick polymer sheet was placed below the stainless steel template which was used to position the circular cylinder at the centre of the platform. However, this limited the use of powder bed preheating in these experiments and therefore the bed temperature was maintained at room temperature. The processing parameters are summarized in Table 9-3. Figure 9-13 shows a schematic of the experiments conducted in this section.

\begin{tabular}{|c|c|}
\hline Processing parameters & Value/ Range \\
\hline Laser power & $5,10,15,20,25,30,35,40,45$ and $50 \mathrm{~W}$ \\
\hline Scan speed & $65 \mathrm{~mm} / \mathrm{s}$ \\
\hline Hatch distance & $80 \mu \mathrm{m}$ \\
\hline Bed temperature & $32^{\circ} \mathrm{C}$ (room temperature) \\
\hline
\end{tabular}

Table 9-3: $\quad$ Processing parameters for temperature measurements 


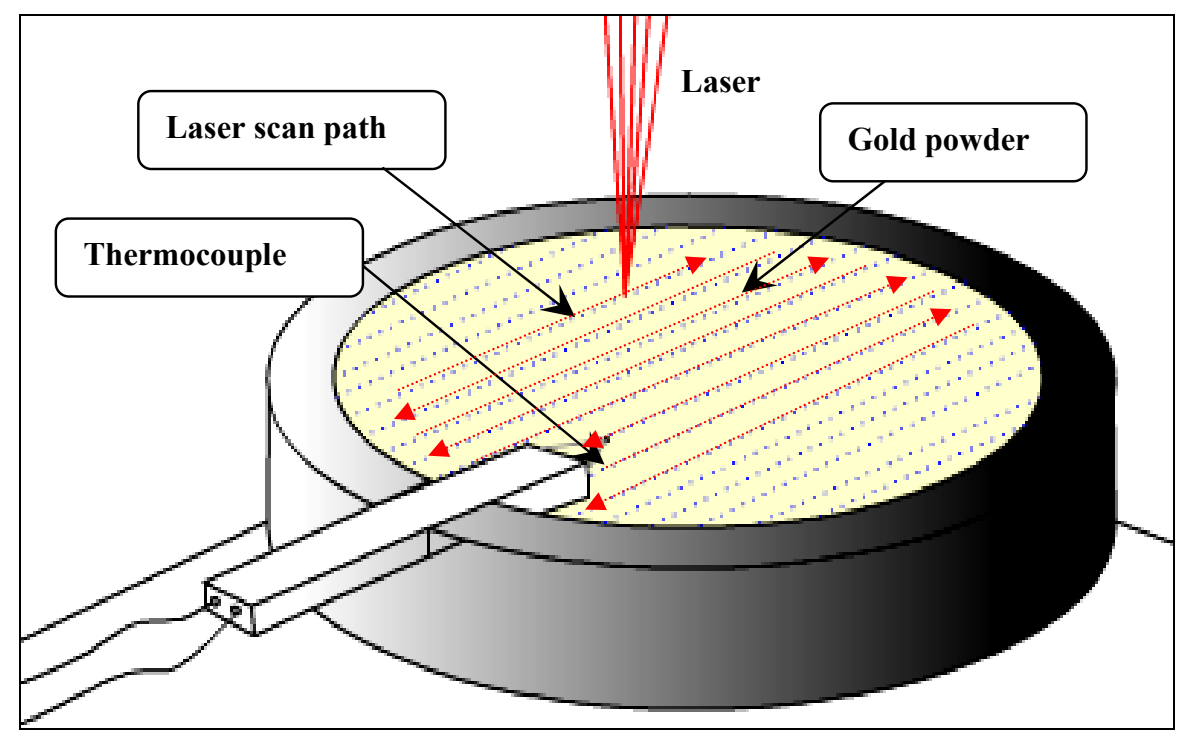

Figure 9-13: Schematics of the thermocouple incorporated into the gold powder bed for temperature measurements

\subsubsection{Results and Discussion}

Figure 9-14 shows the maximum temperature achieved at different laser power. The maximum temperature $\left(550^{\circ} \mathrm{C}\right)$ was noted for a laser power of $50 \mathrm{~W}$. It was observed that the gold powder started melting at a laser power above $20 \mathrm{~W}$. It was also noted that the powder bed temperature would drop to near the room temperature well before the final scanning and therefore, the pre-scan technique was not found feasible for pre-heating the powder bed.

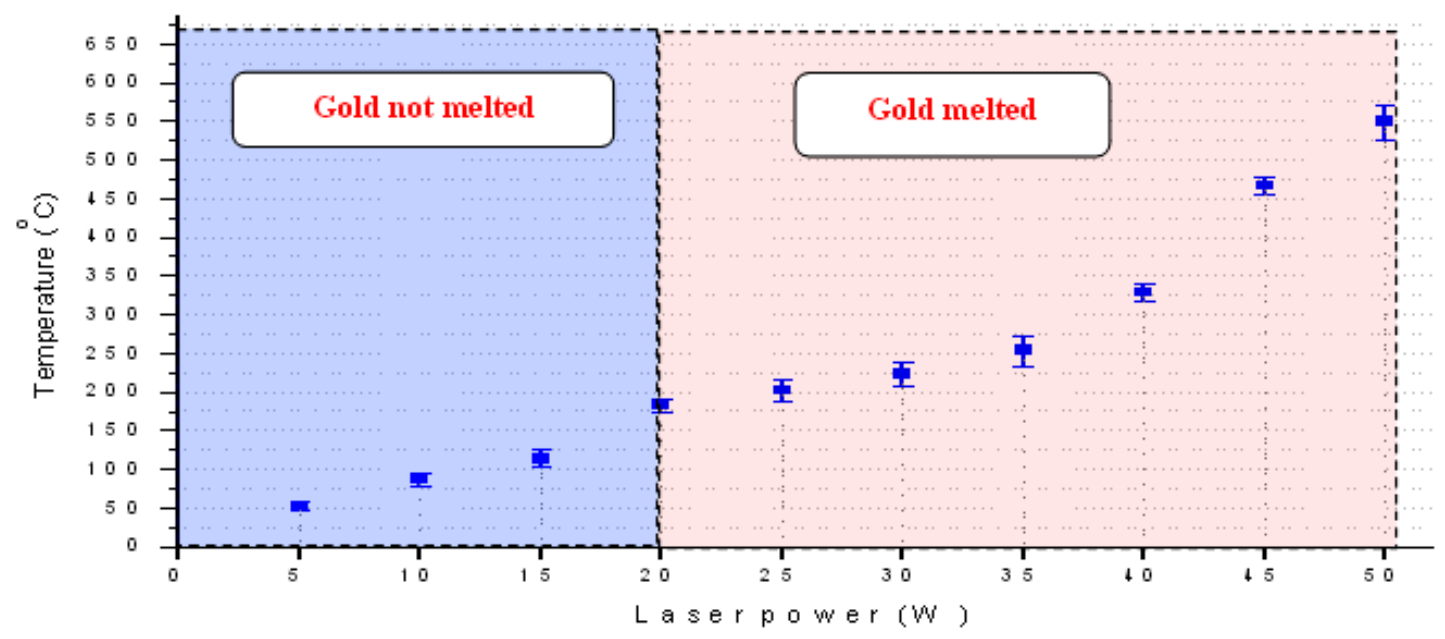

Figure 9-14: Temperature measured by thermocouple at different laser power 


\subsection{Micro Hardness Testing}

The micro hardness testing was performed with a Mitutoyo microhardness testing machine HM 200 series. The gold cubes were mounted in epoxy resin for micro hardness testing. The surface of these samples was already prepared as explained in section 9.1.1. The positioning was performed with an in-built microscope to precisely indent the desired location. Indentations were performed at different loads but at $200 \mathrm{~g}$ the shape of the indentation in the specimen became regular (Figure 9-15). This indicated the load to be suitable for the hardness test. The hardness of gold produced at a scan speed of $65 \mathrm{~mm} / \mathrm{s}$ (HV 29.3) (as shown in Figure 9-16) was found to be higher than that normally expected for 24 carat gold (HV 25).

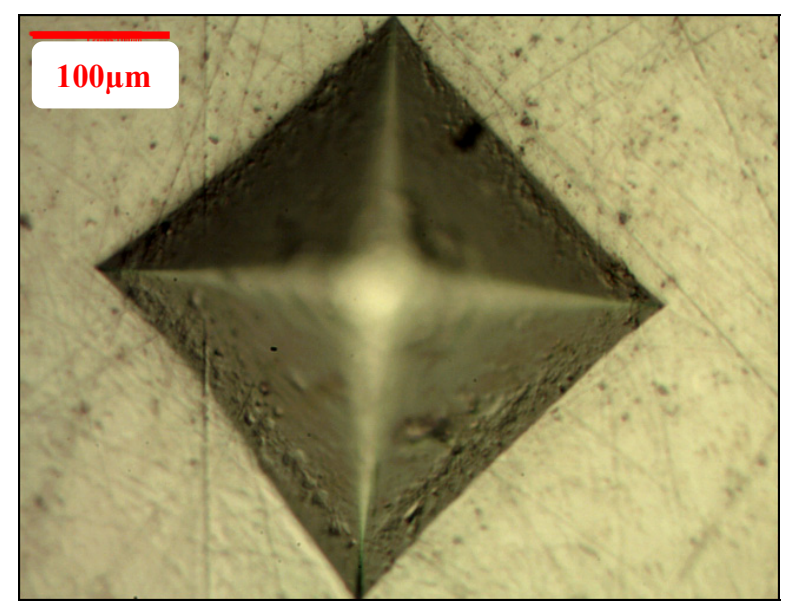

Figure 9-15: Indentation in the gold cube during microhardness testing

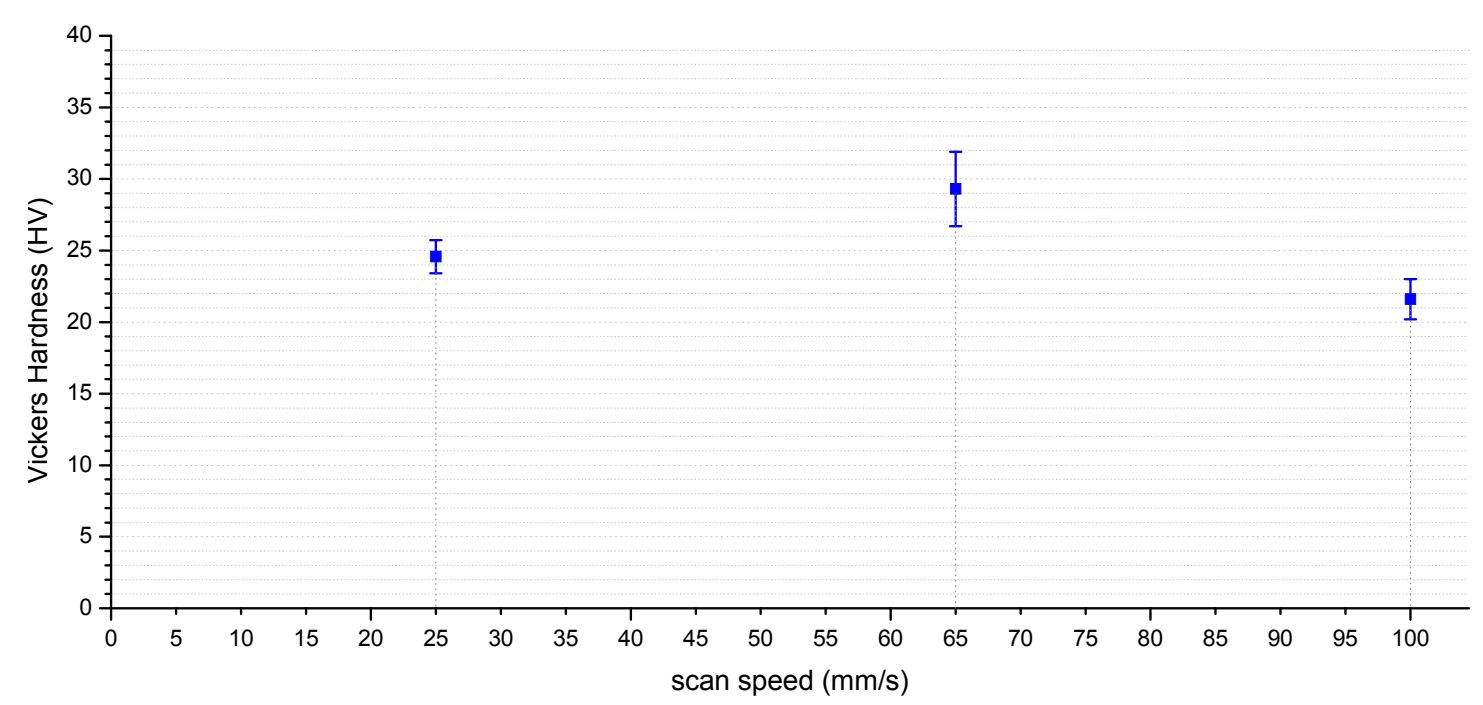

Figure 9-16: Vickers Hardness (HV) for different gold samples 


\subsection{Nanoindentation of Gold Cubes}

Due to the small size of the gold cubes, the nanoindentation method was selected for measuring its elastic modulus. The gold cubes used for nanoindenation were manufacturing using the optimum processing parameters summarized in Table 9-2. Mechanical properties can also be obtained from the experimental load-displacement curve including the elastic modulus and hardness (Fischer-Cripps, 2006)

The indenter used in this work was a three-sided Berkovich indenter. The Berkovich indenter gives the same projected area to depth ratio as the Vickers indenter. The hardness and modulus was calculated by the Oliver and Pharr method (Oliver and Pharr, 2003) in which hardness is determined by measuring peak load divided by the projected area of impression. The elastic modulus was calculated by the reciprocal of the unloading slopes (i.e. compliance) and the estimated plastic depth from the loadingunloading curve.

\subsubsection{Selection of Maximum Load}

In nanoindentation the maximum load has an influence on the properties observed for a material, therefore the selection of maximum load is vital for determining the material properties. Due to the soft nature of pure gold, the load range selected for the gold cubes was from $5 \mathrm{mN}$ to $100 \mathrm{mN}$. Figure $9-17$ shows the effect of load on the hardness measurements obtained at different hold times for the gold samples. The hold time is the time for which the indenter remains in the maximum loading position before it starts unloading. It can be seen in Figure 9-17 that hardness decreased with increasing load and hold time, a behaviour that has been observed for other materials elsewhere (Zhang et al, 2009). The effect of load on the hardness measurement was found to be very small at loads above $50 \mathrm{mN}$. At low loads, the indenter penetrates to a smaller depth in the material and therefore other factors such as indenter size and material surface behaviour plays a larger role in the determination of hardness. But at high loads, the indenter penetrates to a greater depth in the material and these effects are minimized, which are also seen in Figure 9-17. Therefore a maximum load of $50 \mathrm{mN}$ was selected for further experimentation. 


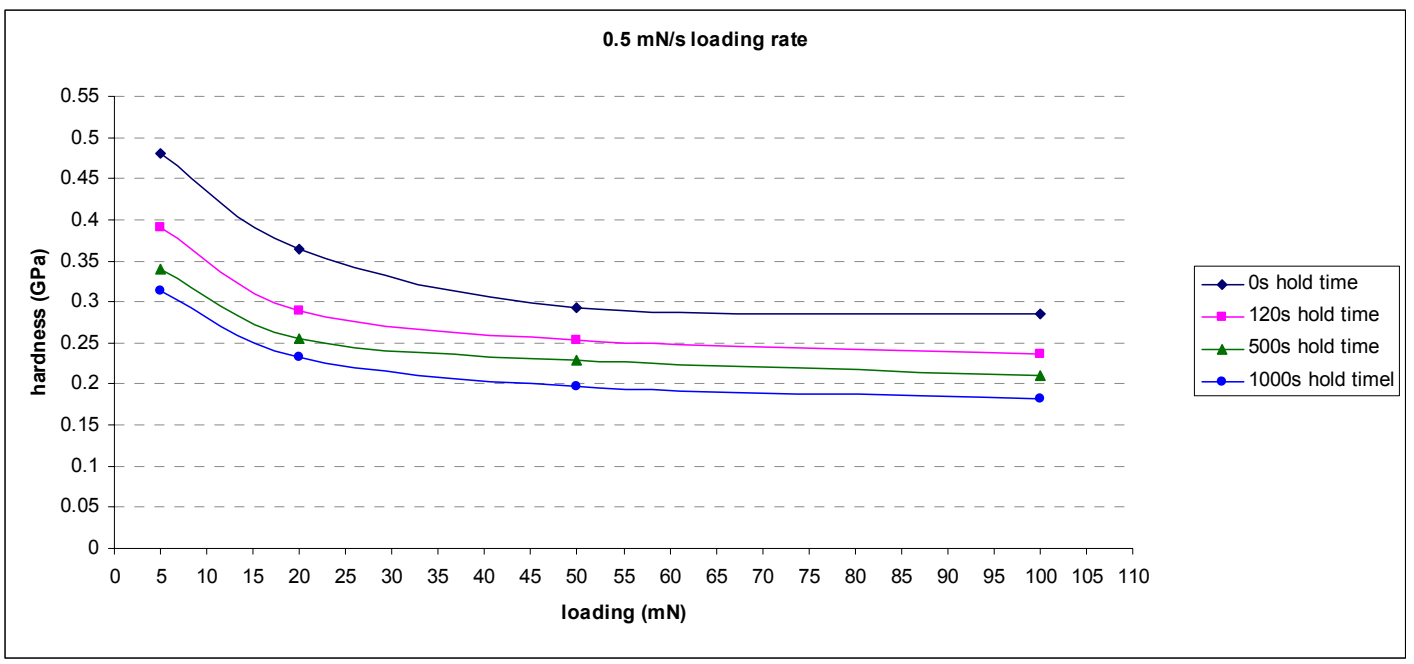

Figure 9-17: Load vs hardness for different hold time

\subsubsection{Selection of Loading Rate and Holding Time}

Due to the time dependant behaviour of gold (Figure 9-17), the samples were also studied for the effect of loading rate on the hardness. Loading rates of $0.5 \mathrm{mN} / \mathrm{s}$ and $1 \mathrm{mN} / \mathrm{s}$ were selected for hardness measurements. Figure 9-18 shows the effect of loading rate on the hardness measurements of gold cubes for different hold times.

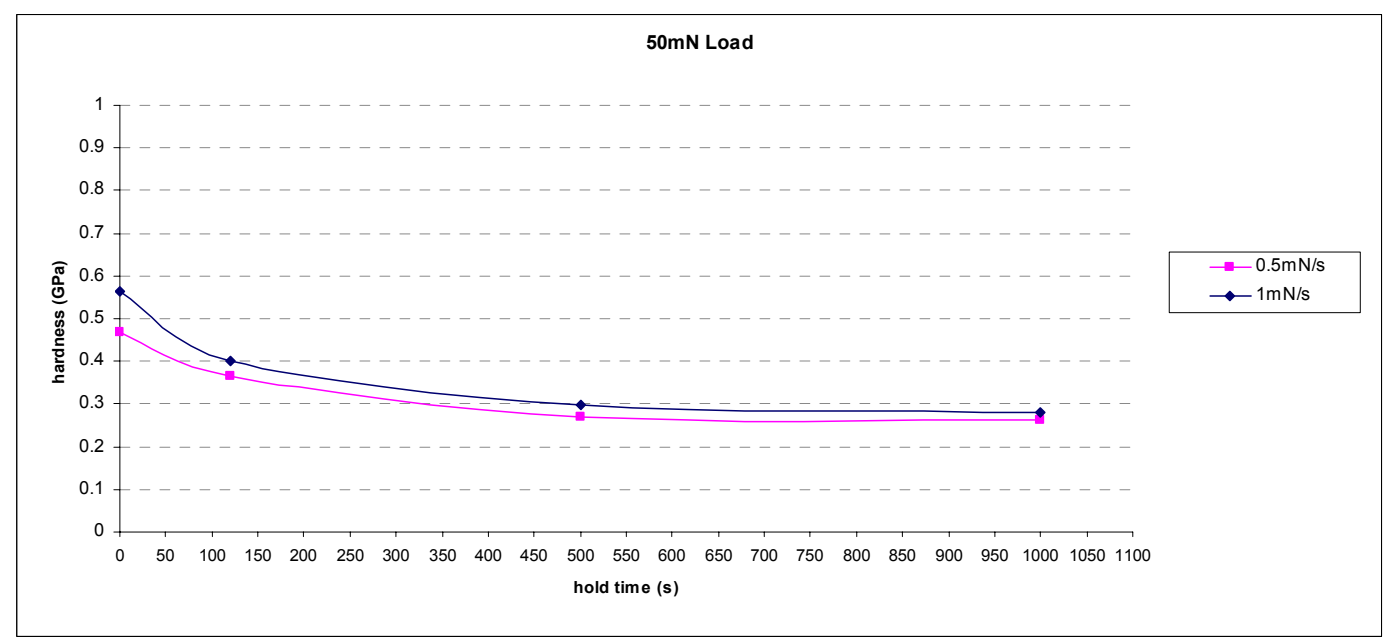

Figure 9-18: Hold time vs hardness for different loading rate

It was observed that a low loading rate tends to give a lower hardness value but as the hold time is increased above $500 \mathrm{~s}$, the difference is almost negligible. In order to eliminate the effect of loading rate and hold time on the final measurements for gold cubes, a loading rate of $0.5 \mathrm{mN} / \mathrm{s}$ and holding time of $500 \mathrm{~s}$ was selected. For a load of $50 \mathrm{mN}$ (selected in section 9.8.1), loading rate of $0.5 \mathrm{mN} / \mathrm{s}$ and a hold time of 500 second, the 
hardness of gold cubes was found out to be $0.271 \mathrm{GPa}$, which is equal to a Vickers hardness of 27.63 .

\subsubsection{Elastic Modulus of Gold Cubes}

The modulus of gold cubes was also obtained for a maximum load of $50 \mathrm{mN}$ and loading rate of $0.5 \mathrm{mN} / \mathrm{s}$. Figure 9-19 shows the modulus of gold cubes for different hold times. There was a decrease in the modulus with increasing hold time but the effect was very small after a hold time of 500s (as previously observed for hardness measurements). The elastic modulus of gold cubes for a hold time of $500 \mathrm{~s}$, loading rate of $0.5 \mathrm{mN} / \mathrm{s}$ and maximum load of $50 \mathrm{mN}$ was found to be $72.5 \mathrm{GPa}$. Gold in pure form has a modulus of $77.2 \mathrm{GPa}$.

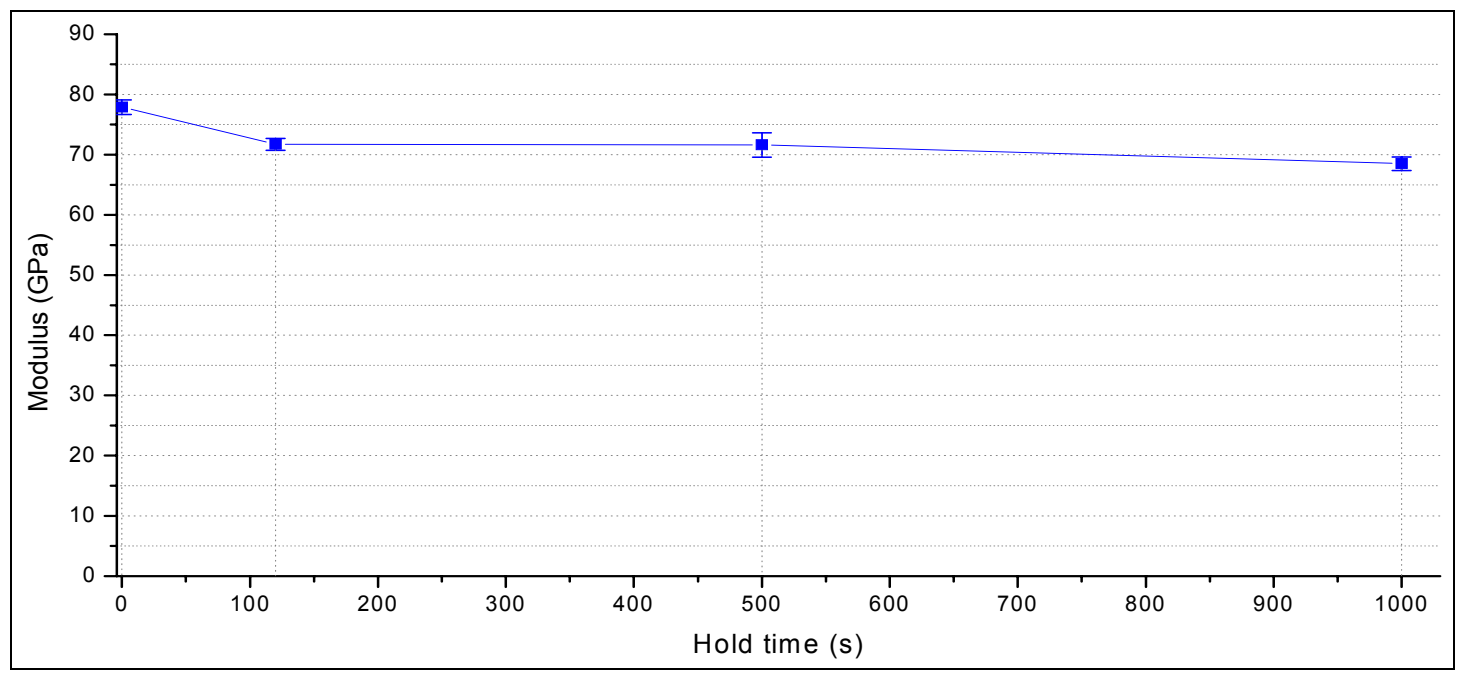

Figure 9-19: Hold time vs modulus for gold cubes

\subsection{Manufacturing of Gold Dental Parts}

The gold dental parts (premolar and molar) were produced from gold powder using the optimum processing parameters summarized in Table 9-2. The small build platform and deposition system was used to make the dental premolar and molar parts. Figure 9-20 shows the build orientation of these dental parts. In order to obtain a smooth surface on the outer and inner sides of the premolar part, it was supported by four 1x1mm supports as shown in Figure 9-20a. The molar part (Figure 9-20b) could not be supported at the very thin edges and therefore it was supported at one point by a cone shaped support. This cone shaped support facilitated easy removal of the part without causing any damage and also kept the thin edges of the part intact during the part removal operation. These dental parts were successfully produced with minimal supports. There was no sink-in into the powder 
during the laser processing and no damage occurred to the unsupported regions of these dental parts, which indicates the optimum parameters selected in section 9.4 to be suitable for dental parts manufacturing with overhanging structures or unsupported geometries. Sink-in is observed during the laser processing when extra energy is being delivered to the powder which makes the melted part sink into the powder underneath especially if it is not supported.
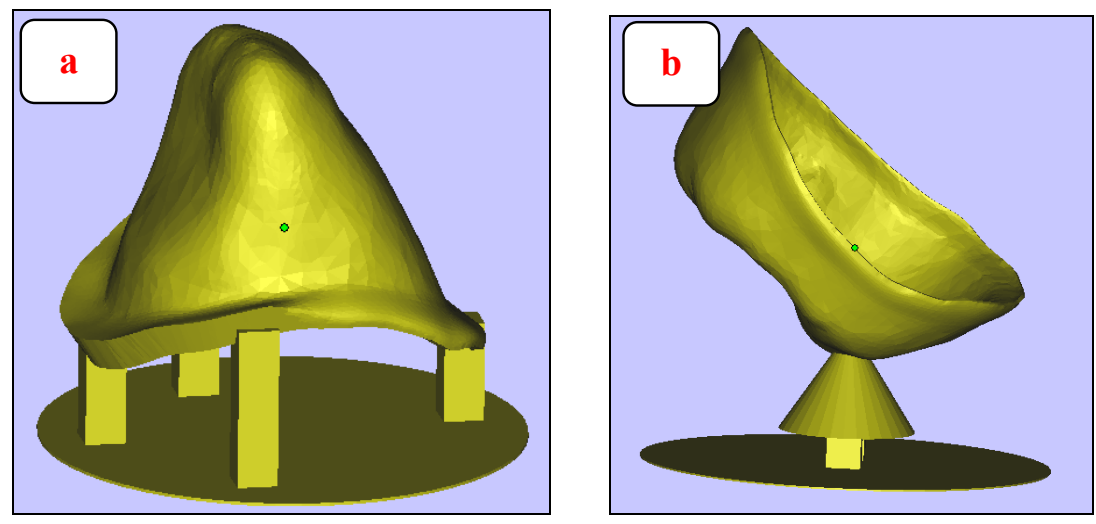

Figure 9-20: Build orientation of (a) premolar and (b) molar dental parts

The gold premolar dental parts were produced with two different layer thicknesses i.e. 75 and $50 \mu \mathrm{m}$ (Figure 9-21) to check the effect of layer thickness on the surface quality of these parts. The dental part with $75 \mu \mathrm{m}$ layer thickness showed some layer delamination at different points, while the part with $50 \mu \mathrm{m}$ layer thickness was observed to have no such problems. The effect of layer thickness on the density of gold cubes has already been discussed in section 9.2.4.

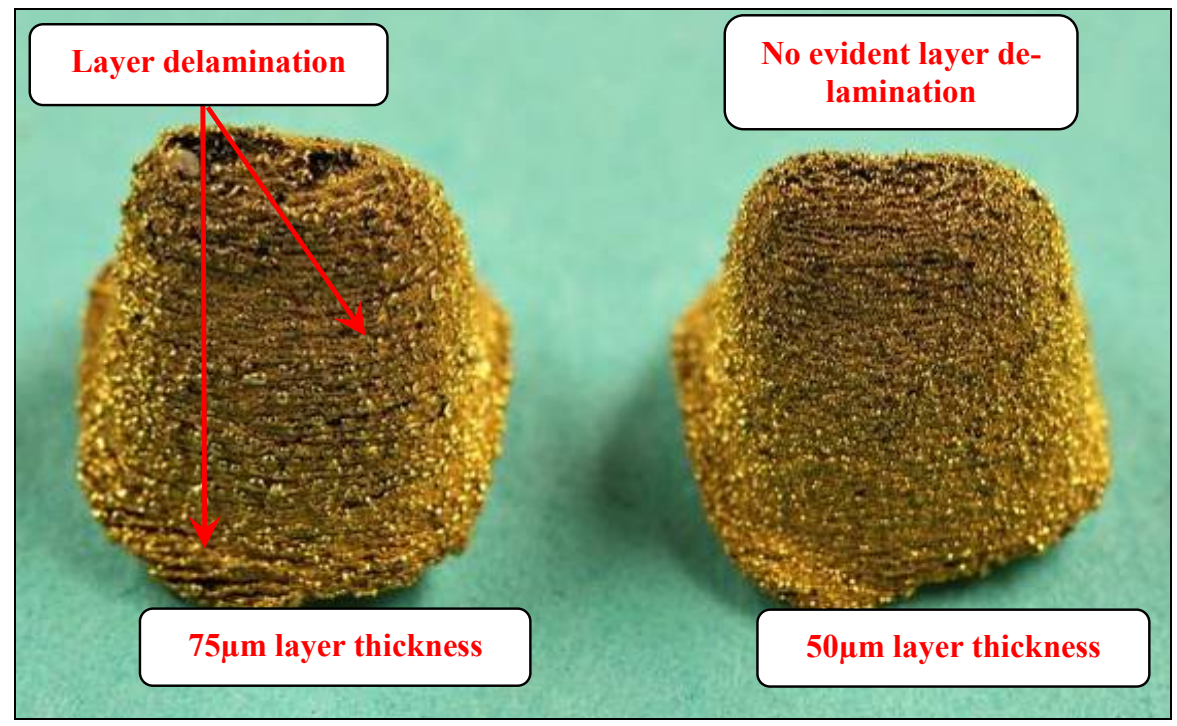

Figure 9-21: Gold premolar dental parts produced with $75 \mu \mathrm{m}$ and $50 \mu \mathrm{m}$ layer thickness 
Figure 9-22 and 9-23 shows different views of the gold premolar and molar dental parts which were manufactured using the optimum parameters specified in Table 9-2. The gold premolar part was damaged during its removal from the substrate.
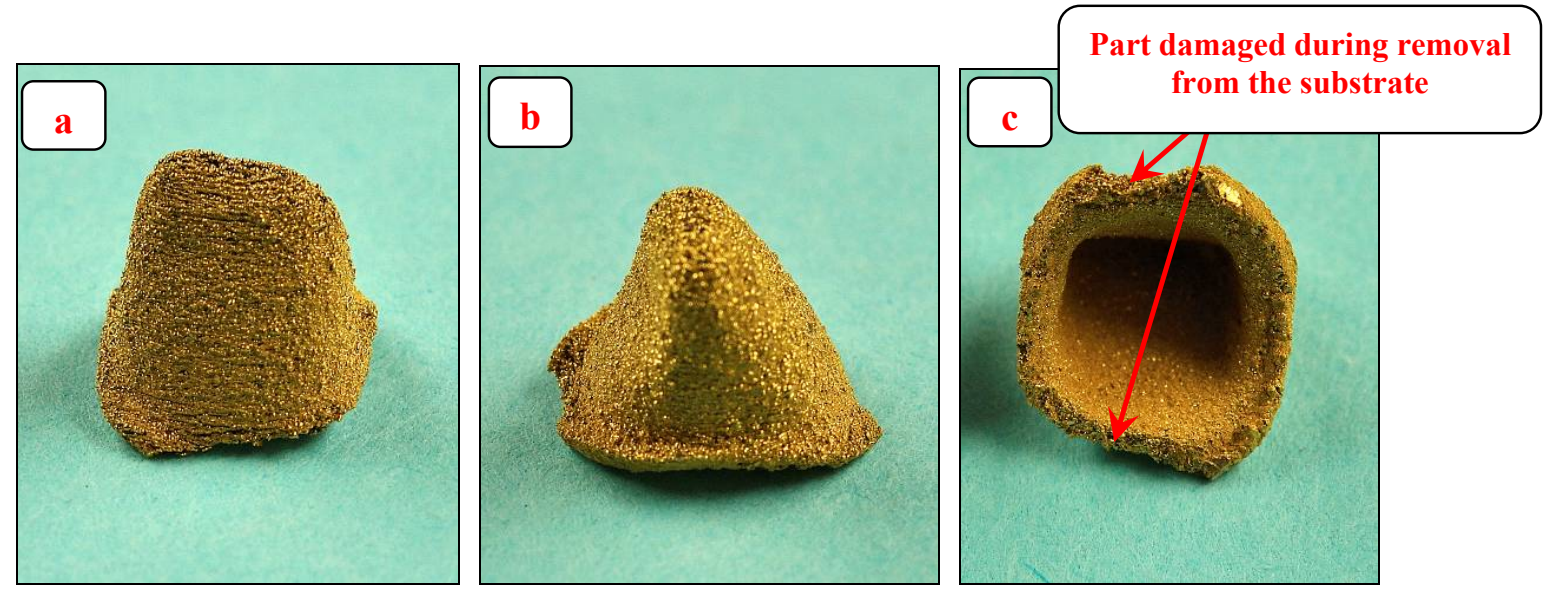

Figure 9-22: (a-c) Different views of gold premolar dental part
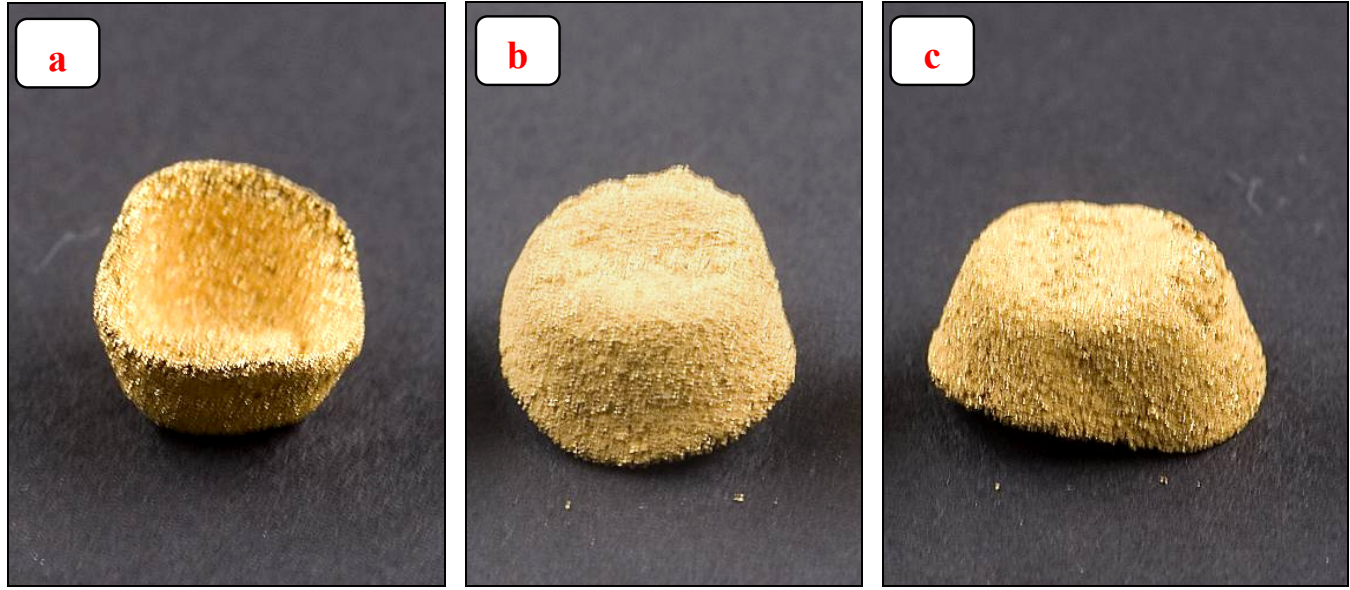

Figure 9-23: (a-c) Different views of gold molar dental part

\subsection{Computerised Tomography (CT) Scan of the Dental Parts}

The gold cubes were analyzed for density in sections 9.1 and 9.2 but in order to check the density of premolar and molar dental parts, a non destructive testing technique namely Computerised Tomography (CT) scan was used. The micro CT scan is similar to the conventional CT scans used in medical imaging, but with capability to capture object with much more detail (up to micron sized features). In this method, X-rays of different intensities are fired at the sample. A detector plate opposite to the X-ray head captures the $\mathrm{X}$-rays that have passed through the part. The sample holder is capable of rotating through $360^{\circ}$. Before scanning the part, the micro CT machine was calibrated by firing X-rays at the detector plate without the part. This initial calibration gave the intensity of the X-rays 
within the chamber i.e in the presence of air in the chamber. During the scanning process, $\mathrm{X}$-rays were fired at the sample placed in the sample holder. Some of these X-rays were absorbed by the sample while others passed through with varying degree of absorption and collected by the detector plate. The X-rays collected by the detector were normalized against the initial calibration data to obtain the cross-section of the sample. Multiple crosssection images of the sample were taken and merged together to obtain a 3-dimensional model.

A major problem with gold is its opaque nature to X-rays. The X-rays can only penetrate through a very thin layer of gold. After a few trial runs, it was observed that due to the thickness of the dental premolar part, the X-rays could not penetrate through it. However, the dental molar part was successfully scanned but with poor quality. This indicates that the X-rays collected by the detector after passing through the molar part were not strong enough to make a detailed model. A greyscale level in the software (installed on the micro CT machine) was used to manually differentiate between the part boundary and the surroundings but due to the poor quality of the scans, it was not clear. Therefore, an accurate 3D model could not be created.

Figure 9-24 (a-d) shows CT scans at different sections for the molar part. The solid part is identified as the grey area whereas the porosity is shown as black spots within the grey area. Although the porosity could be seen in individual scans the internal part porosity could not be quantified accurately due the poor quality of the results. 

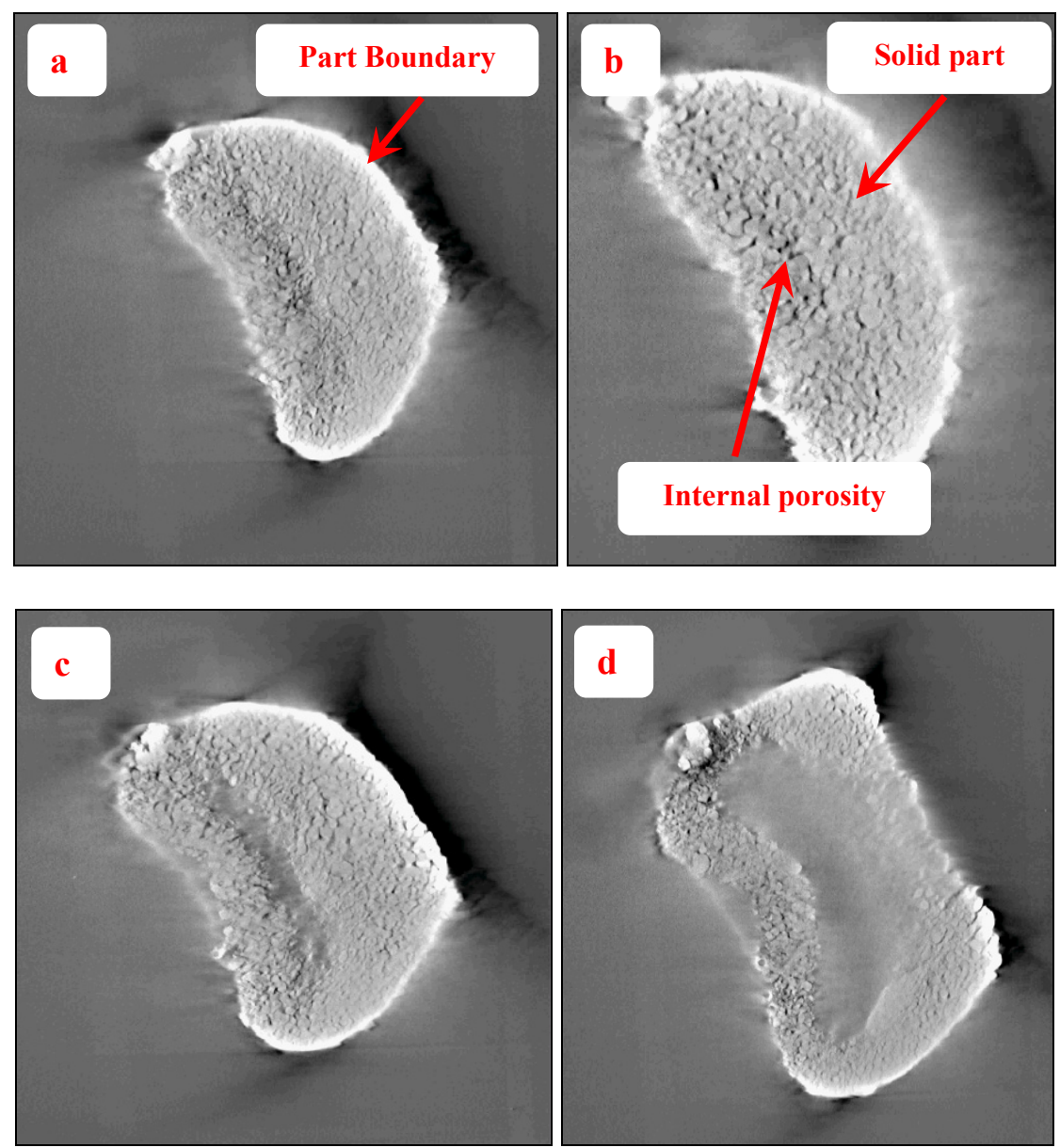

Figure 9-24: (a-d) Computerised Tomography (CT) scans at different sections of gold molar part

\subsection{Porosity Measurement of Gold Dental Part}

The gold dental premolar part was also checked for its internal porosity by the destructive testing technique. The gold premolar part was cross-sectioned and its porosity measured by performing image analysis on the cross sectional image. The internal porosity was measured at three different sections of the premolar part. Figure 9-25 shows crosssection image of the dental premolar part. Figure 9-26 shows cross-section images of gold premolar part at different locations within a cross-section. The internal porosity in the premolar part is quite evident in these cross-section images. The average porosity of the gold premolar part was $7.2 \%$, which was less than the minimum porosity obtained for gold cubes (section 9.2.4). This could be because of the difference in shape of the two parts, where the premolar part is a thin feature part and cube is a solid part and needs further investigation. 


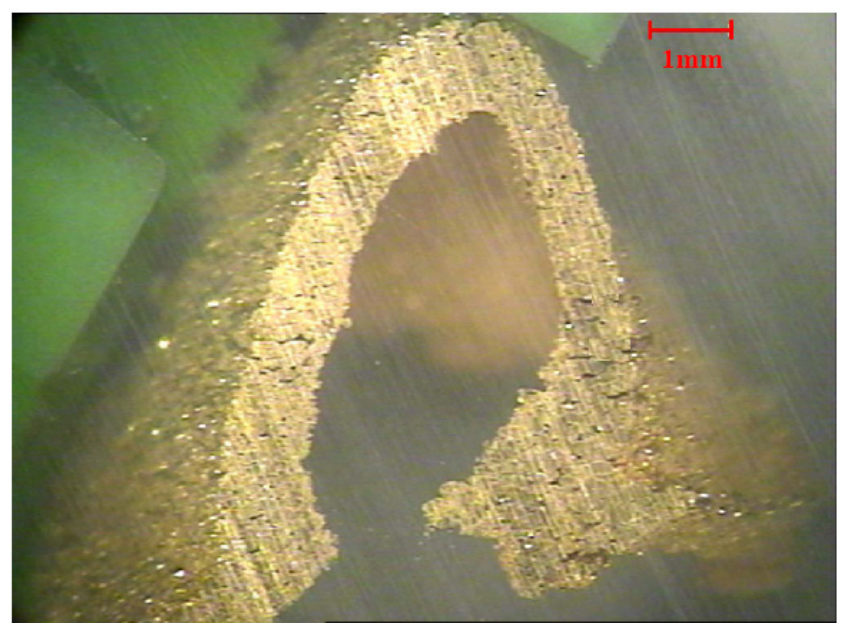

Figure 9-25: Optical image of the cross-section of gold premolar part
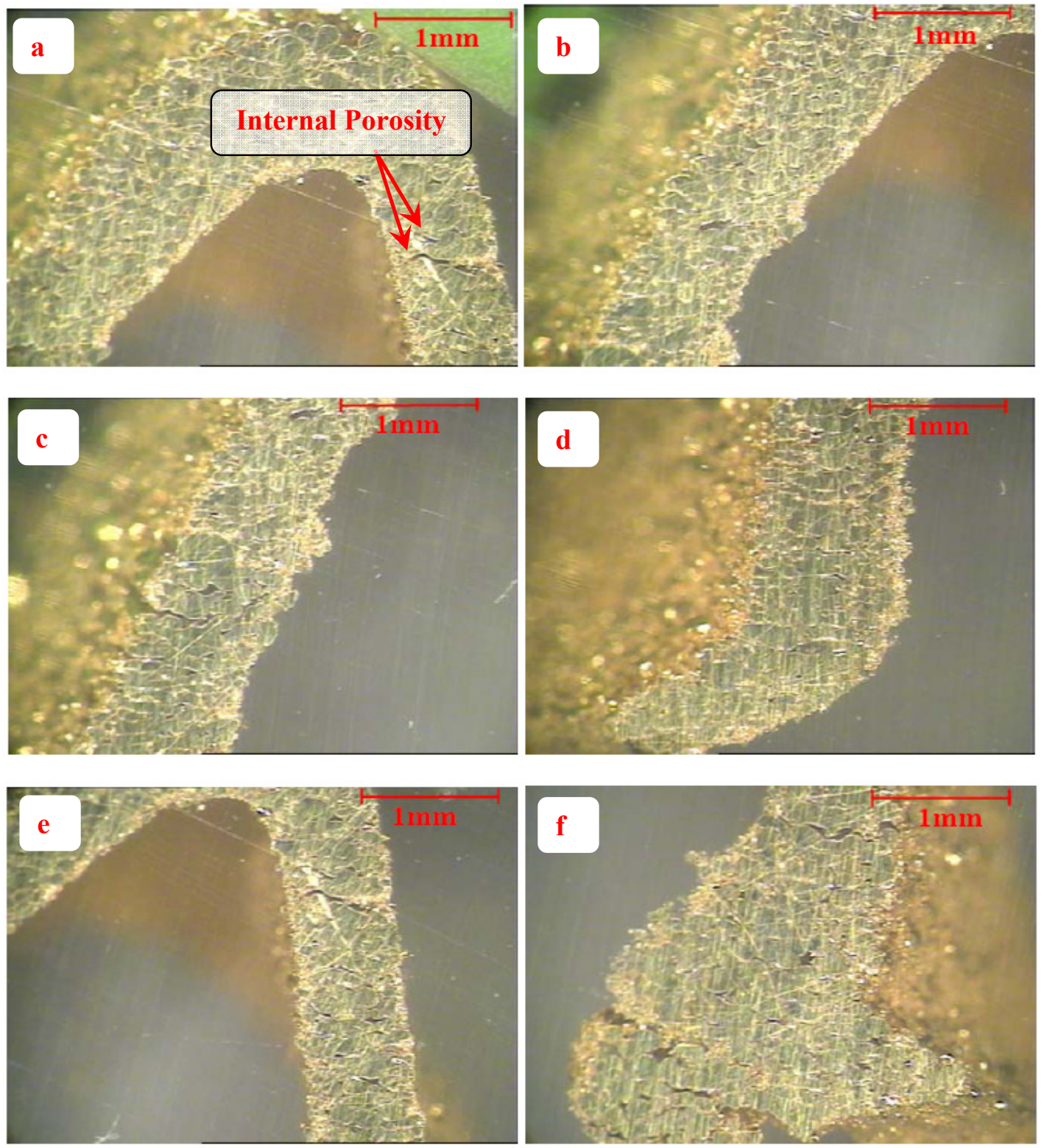

Figure 9-26: (a-f) Cross-sectional images of gold premolar part at different locations 


\subsection{Summary}

In this chapter the gold cubes were analysed for their internal porosity. The gold cubes produced with the optimum parameters identified in Chapter 8 (Figure 8-2) were found to have a minimum porosity of approx $12.5 \%$. In this analysis, it was observed that the majority of the porosity was inter layer rather than intra layer which could be due to the lack of energy transferred to the previous layer of gold. The low intra layer porosity indicated the good layer quality for these parameter settings. In order to improve the energy density supplied to gold powder, the layer thickness and hatch distance were varied. Reduction of layer thickness form $100 \mu \mathrm{m}$ to $50 \mu \mathrm{m}$ resulted in reduction of average porosity from $14.4 \%$ to $10.4 \%$, which was mainly due to the reduction in the gap between the two layers. However, the reduction in hatch distance had a negligible effect on porosity of the gold cubes. The porosity was found to be a minimum $(7.7 \%)$ for a laser power of $50 \mathrm{~W}$, scan speed of $65 \mathrm{~mm} / \mathrm{s}$, layer thickness of $50 \mu \mathrm{m}$ and hatch distance of $80 \mu \mathrm{m}$. Microhardness and nanoindenation tests were also performed to check the mechanical properties of the gold cubes.

The heat transfer in the gold powder bed was also measured and the feasibility of a pre-scan as a preheating technique was checked. It was observed that the powder bed did not remained heated for longer enough for the laser to scan the next layer with high power to melt it while the powder was still in a heated state from the pre-scan.

The gold dental parts (premolar and molar) were manufactured using the optimum parameters. These gold dental parts were also inspected for internal porosity by a micro CT machine. The gold premolar part was found to be too thick for the X-rays to penetrate; hence it was not possible to scan the premolar part. Although, the gold molar part was successfully scanned, due to poor quality of the scan, porosity could not be quantified accurately. The internal structure of the molar part in CT scan was observed to have some porosity. The gold premolar part was cross-sectioned and examined for internal porosity. The average porosity of the premolar part (7.2\%) was found to be less than that observed for gold cubes $(7.7 \%)$. This could be due to the difference in the shape of the parts as the gold premolar part was a thin wall structure whereas the gold cubes were fully solid parts. 


\section{Chapter 10}

\section{CONCLUSIONS AND RECOMMENDATIONS FOR FUTURE WORK}

This research was aimed at understanding the effect of processing parameters for SLM of 24 carat gold. A comprehensive literature survey revealed that very little work had been published on the powder properties and Selective Laser Melting (SLM) of gold powder. A series of experiments performed was related to the material properties of the gold powder, identification of suitable process parameters for its laser processing and obtaining mechanical properties of parts produced from 24 carat gold powder.

Various experiments were carried out in order to identify the material properties of gold powder which included radiation absorption, Particle Size Distribution (PSD), apparent density and tap density. The radiation absorption analysis of gold powder was useful in understanding the melting behaviour of gold powder during processing in Selective Laser Melting (SLM). Based on the results from density experiments, a new deposition system was designed to (ease powder flowability and) spread single layers of gold powder for multiple layer part manufacturing. The laser melting of gold showed five different processing regions: balling, good melting, unstable melt, weak sintering and very little sintering. The balling phenomenon was observed (at certain parameters) which was related to the melt pool instability at those processing parameters. The good melting region produced good continuous gold beads and these parameters were subsequently used to produce single layers and multiple layer gold parts. These gold cubes were analyzed for their internal porosity and mechanical properties. Gold dental parts (premolar and molar) were successfully manufactured using the optimum processing parameters. Following the list of objectives (see Chapter 4), this $\mathrm{PhD}$ research study has brought the results described below.

\subsection{Material Characterisation}

\subsubsection{Reflectivity of Gold Powder}

The gold powder was first analyzed for its reflectivity in the infrared range using the DRIFTS technique. The absorption of radiation by the powder was found to be different to material in bulk (sheet or block form), where multiple reflections of the 
radiation within small pores in the powder bed increased its radiation absorption by multiple reflections as also observed for other metals (nickel alloy) elsewhere (Tolochko et al, 1997). The gold powder was compared with metal powders such as H13 tool steel, stainless steel, copper and Al 12 Si powders. The comparison revealed that gold powder was more than twice as reflective as commonly processed (by SLM process) metal powders such as stainless steel and H13 tool steel powder. A high reflectivity of gold powder indicated less radiation absorption and hence less energy transferred to melt it. The fact that gold powder reflected $85 \%$ of the incident radiation (Figure 5-5) was useful in explaining the inter-layer porosity in the multiple layer parts (Chapter 9). A higher packing density of the gold powder was observed to have higher reflectivity compared to the loosely packed gold powder. This was due to the reduction in pores between the particles in the tightly packed powder bed.

\subsubsection{Particle Size Distribution (PSD)}

The PSD analysis of the gold powder was first performed in distilled water and then in isopropanol. The PSD analysis in distilled water indicated larger particle size than the isopropanol. This was found to be due to the non-dispersing nature of the gold powder in water. The mean particle size of the gold powder was found to be $24.5 \mu \mathrm{m}$ in isopropanol. The SEM images of gold powder also confirmed the smaller particles of gold powder sticking together and forming larger agglomerates. These agglomerates of gold powder did not disperse in water and thus indicated a larger particle size. Therefore, the PSD in isopropanol was taken to be the actual PSD of gold powder. The gold powder was found to be cohesive in nature. This cohesive nature resulting in agglomerations in the gold powder reduced the powder flowability.

\subsubsection{Apparent and Tap Density}

Apparent density of the gold powder was tested using the Carney and Hall funnels but due to the cohesive nature of the gold powder, it did not flow through them. Therefore, according the ASTM B329 (ASTM B329, 1998), BS EN 23923-2 (BS EN 23923-2, 1993) and ISO 3923-2 (ISO 3923-2, 1981) standards, apparent density experiments were performed using the Scott volumeter. The apparent density of gold powder showed it to be non-free flowing in nature. The tap density of gold powder was calculated by two different methods i.e. Constant Weight Tap Density (CWTD) and Constant Volume Tap Density (CVTD). For gold powder, the difference in these methods was found to be almost negligible. 


\subsection{Processing}

\subsubsection{Experimental Setup}

Due to the small amount of material available and the cohesive nature of the gold powder, a new deposition system and a small build platform were designed. Three different hoppers were designed and based on the layer quality and repeatability, the suitable one was selected. The build volume of the smaller build platform was $15 \mathrm{~mm}$ in diameter and $20 \mathrm{~mm}$ in depth, which was enough to produce gold cubes and gold dental parts. The new platform and deposition system was installed in the existing SLM 100 system. The laser spot size measurement was performed using the black burn paper. The minimum spot size was found to be approximately $35 \mu \mathrm{m}$.

\subsubsection{SLM of Gold}

\section{Single Scans}

Single scans were performed on a $100 \mu \mathrm{m}$ layer of gold powder to identify suitable processing parameters. From the processing window, five different regions were identified i.e balling, good melting, unstable melt, weak sintering and very little sintering regions.

As explained in detail in section 2.3.3.3, if the total surface of the melt pool becomes larger than that of a sphere of the same volume then the melt pool becomes unstable and splits into smaller entities (balling). Interestingly, the balling phenomenon was observed in two different regions in the processing window. The balling at low scan speed could have been due to the high energy input and more time available for the molten metal to split and form larger droplets. At high scan speeds, the balling could be due to the increase in the melt pool length and reduction in its width, thus destabilizing the melt pool (L/D > 2.1) (Kruth et al, 2004).

The size of the droplets in the balling regions was also observed to increase with decreasing scan speed and increasing laser power and visa versa. This was related to the increase in break-up time of the molten metal in the melt pool. At high laser power and low scan speeds (i.e. at high energy input) a larger melt pool was created by melting more gold powder. This larger melt pool had more time in the molten state (increased break-up time) and thus was affected to a greater extent by the thermocapillary flows (Marangoni flow and surface tension gradients) (see Section 2.3.3.3). Due to these factors the larger melt pool split into larger droplets. At relatively low laser power and high scan speeds (i.e. low energy input), the size of the melt pool created was relatively small. The instability of 
tracks at these speeds due to the increase in L/D ratio over 2.1 resulted in the smaller melt pool splitting into relatively smaller droplets.

Due to the balling, the multiple layer gold parts produced with these parameters (see Chapter 9) showed weak inter-layer adhesion, internal porosity in parts and nonuniform layers as also observed by Fuh et al (1995), Hauser et al (2005) and Kruth et al (2003) for other metals. The non-adhesion of gold to the underlying substrate was another factor which assisted in balling, which has been seen with stainless steel powder (O'Neill et al, 1998).

In the good melting region, the gold powder was fully melted and continuous beads were produced. The good melting region was found to be very narrow and therefore the processing window needed to be further expanded for high laser power (above 50W). As observed in a similar situation (Kruth et al, 2004), the good melting region could become wider thus enabling a broader selection of processing parameters in this region. In the unstable melt region, although the gold powder was completely melted, the melt pool destabilized and spread in different directions resulting in no continuous beads. In the weak sintering and very little sintering regions, the gold powder could not be melted completely, these regions were not considered important for further analysis in this research.

\section{Single Layers}

From the processing window, suitable processing parameters were selected to produce single layers. The first line balling was observed in single layer scans at low scan speeds and high laser power i.e. high energy input. The first line balling could have been due to the powder available on both sides of the first laser scan on the gold powder layer. This created a larger melt pool and due to the low scan speeds, enough time was available for the melt pool to split and form balling. For subsequent scans, the powder was only available on one side of the laser scan hence reducing the melt pool size and preventing the balling to occur.

\section{Multiple Layers Parts}

The suitable processing parameters identified in single scan and single layers were successfully used to produce gold cubes. The new build platform and powder deposition system was used to produce these gold cubes. 


\subsubsection{Manufacturing of Gold Dental Parts}

The gold dental parts (premolar and molar) were successfully manufactured with the optimum processing parameters using the new build platform and powder deposition system. The gold premolar part produced with $50 \mu \mathrm{m}$ layers showed clear improvement in layer delamination compared to the premolar part produced with a $75 \mu \mathrm{m}$ layer thickness.

\subsection{Analysis}

\subsubsection{Internal Porosity}

The gold cubes were analyzed for their internal porosity by a cross-sectioning technique. The internal porosity was found to be at minimum for parameters representing the good melting region i.e. $50 \mathrm{~W}$ and $65 \mathrm{~mm} / \mathrm{s}$. The gold cubes produced with parameters belonging to the balling and unstable melt regions showed more porosity due to the nonuniform layers and poor adhesion. The majority of the porosity in the gold cubes was interlayer, which was due to the high reflectivity of gold powder and hence less heat transfer to the region between the layers. The inter-layer porosity was reduced by decreasing the layer thickness from $100 \mu \mathrm{m}$ to $50 \mu \mathrm{m}$. This could be due to the thinner layers which required less energy to melt and fuse it to the previous layers. The hatch distance had a negligible effect on the inter-layer porosity of the gold cubes. The reduction in the hatch distance increased the energy delivered but it was still not enough to completely melt the gold powder and fuse it to the previous layer.

The gold dental parts (premolar and molar) were first tested for their internal porosity using CT scans but due to the poor quality of the scans, the internal porosity of these parts could not be quantified. The gold premolar part was then tested for its internal porosity by cross-sectioning technique. The internal porosity in the premolar part was found to be less than the gold cubes, which could be due to the difference in structure of these parts and needs further investigation.

\subsubsection{Mechanical Properties}

The gold cubes were also tested for mechanical properties (hardness and modulus). The average hardness was found to be HV 29.3 which was more than normally expected for 24 carat gold (HV 25). Due to the small size of the gold cubes, the modulus was obtained by the nanoindentation technique. The modulus of gold cubes was found to be $72.5 \mathrm{Nm}$, which was lower than that of 24 carat gold (7.2 GPa). 
The difference in hardness and modulus of gold cubes compared to the 24 carat gold could be due to the rapid heating and cooling during the laser processing or the presence of internal porosity in these parts. However, this needs further investigation at the microstructural level.

\subsubsection{Powder Bed Pre-Heating}

A pre-scan technique was also tested as a powder bed preheating technique. In this technique, a first scan at a low laser power would pre-heat the powder layer thus acting as a powder bed preheating and a subsequent scan at high laser power would melt the gold powder. Due to the rapid drop in temperature, this technique was not found suitable to be used as powder bed preheating technique.

\subsection{Specific Conclusions}

- The gold powder was very reflective (reflectance near 85\%) compared to other metals being processed by SLM such as stainless steel and H13 tool steel (reflectance near 40\%). This means that high laser power is required to melt the gold powder.

- The powder with a smaller particle size had lowest reflectance due to the possibility of many small gaps between the smaller particles in the powder bed which act as black bodies.

- The reflectance of the gold powder was also higher with a higher packing density and so again a higher laser power is required to melt it.

- The gold powder was cohesive and non free flowing in nature. This hindered the powder deposition during processing in the SLM system, which necessitated the development of a new deposition system.

- The gold powder was checked for its Particle Size Distribution (PSD). The gold powder had a better dispersion in isopropanol solution as compared to distilled water. The mean particle size for the gold powder was $24.5 \mu \mathrm{m}$.

- The gold powder was analyzed for its apparent and tap densities. The different aspect ratio density cups had a negligible effect on the apparent density of the gold powder $\left(9.5 \mathrm{~g} / \mathrm{cm}^{3}\right)$. The tap density of gold powder was measured by two different 
methods i.e. Constant Weight Tap Density (CWTD) and Constant Volume Tap Density (CVTD). The CWTD and CVTD tap density of gold powder was found to be almost the same $\left(10.3 \mathrm{~g} / \mathrm{cm}^{3}\right)$.

- The gold powder was processed successfully for the first time using the SLM 100 machine. A good processing window was identified with other processing regions such as balling, unstable melt, weak sintering and very little sintering regions. The scan speed of $65 \mathrm{~mm} / \mathrm{s}$ and laser power of $50 \mathrm{~W}$ were selected from the single scan experiments as the suitable parameters for multi-layer part manufacturing. However, a very small processing region was found within the parameters available.

- Gold cubes were manufactured using the new deposition system and build platform. The average internal porosity of these gold cubes was approximately $12 \%$ which was further reduced to approximately $10 \%$ by changing layer thickness from $100 \mu \mathrm{m}$ to $50 \mu \mathrm{m}$. This is because thinner layers require less energy to melt them and allowed better bonding to the previous layers.

- The minimum porosity of gold cubes (7.7\%) was observed for the optimum processing parameters: laser power of $50 \mathrm{~W}$, scan speed of $65 \mathrm{~mm} / \mathrm{s}$, bed temperature of $100^{\circ} \mathrm{C}$, layer thickness of $50 \mu \mathrm{m}$ and hatch distance of $80 \mu \mathrm{m}$.

- The major part of internal porosity in the gold cubes was inter-layer porosity rather than intra-layer porosity.

- The hardness (HV 29.3) of gold cubes was more that of the 24 carat gold (HV 25) while the modulus of gold cubes $(72.45 \mathrm{GPa})$ was less than normally expected for 24 carat gold $(77.2 \mathrm{GPa})$.

The gold premolar part was cross-sectioned and examined for internal porosity. The average internal porosity of gold premolar part was $7.2 \%$. 


\subsection{Recommendations for Future Work}

In this research, 24 carat gold was processed for the first time using SLM. Constrained by a number of factors (such as quantity and high reflectance of the gold powder), certain significant results were obtained as discussed in detail and concluded earlier. A number of parameters like laser power, layer thickness, powder bed temperature etc. were found to affect the experimental outcomes. Based upon these, certain recommendations for any future endeavours in this field are made.

The internal porosity in gold parts could be further reduced by reducing the layer thickness below $50 \mu \mathrm{m}$ as observed in Section 9.2.4, which was limited by the new deposition system in this research. The powder bed preheating is known to reduce the energy required to melt the metal (see Section 2.3.3.2). In this research the bed temperature was limited to $100^{\circ} \mathrm{C}$ (see Section 7.5.7), a higher bed temperature could reduce the energy requirement and therefore reduce the inter-layer porosity. Also, a maximum laser power of $50 \mathrm{~W}$ was used to produce gold cubes but a higher laser power could increase the energy supplied to the gold powder and it could reduce the internal porosity in these parts. Apart from that, at high laser power, the good melting region could get wider (as explained in detail in Section 8.1.2), which will increase the number of good processing parameters and hence optimum processing parameters at this high laser power.

Some of the potential areas for future work are mentioned below:

- The proportion of smaller particles in the gold powder should be varied to improve its flowability, which could eliminate the need for a new hopper and deposition system.

- A high power laser system (above $50 \mathrm{~W}$ ) could be used to process gold powder. It would be useful to check the effect of a high laser power on the good processing window for gold powder and also its effect on the part porosity. As concluded in this research work, most of the porosity was between the layers, so a high laser power could give enough energy to further reduce or completely eliminate the inter layer porosity.

- A suitable alloying metal could be added to the 24 carat gold powder to improve its wettability and observe its effect on the balling phenomenon. This could also be helpful in reducing the balling phenomenon. 
- A new build platform could be developed which has the capability to reach higher bed temperatures (above $100^{\circ} \mathrm{C}$ ). This would reduce the overall energy requirement to melt the gold powder and could also improve the density of the gold parts.

- Some arrangements need to be made to incorporate a high speed thermal camera into the SLM 100 system to study the melt pool dynamics and the balling phenomenon in detail for SLM of gold powder. This study could be helpful in fully understanding and eliminating the balling.

- EDX analysis could be performed on the sintered gold cubes to check for the presence of oxide films which could be responsible for the layer delamination. 


\section{REFERENCES}

3M ESPE (2009), LAVA Chair Side Oral Scanner, [WWW] http://solutions.3m.co.uk/wps/portal/3M/en_GB/LavaCOS/3MESPE-LavaCOS/

[Accessed on $15^{\text {th }}$ April 2009]

Abdullah E. C. and Geldart, D. (1999), The Use of Bulk Density Measurements as Flowability Indicators, Powder Technology, Vol. 102, pp. 151-165, ISSN: 00325910

Abe, F., Osakada, K., Kitamura, Y., Matsumoto, M. and Shiomi, M. (2000), Manufacturing of Titanium Parts for Medical Purposes by Selective Laser Melting In Proceedings of the 8th International Conference on Rapid Prototyping, Tokyo, Japan , 12-13 June, pages 288-293

Abe, F., Osakada, K., Shiomi, M., Uematsu, K. and Matsumoto, M. (2001), The manufacturing of hard tools from metallic powders by selective laser melting, Journal of Materials Processing Technology, Vol. 111, Issue 1-3, pp. 210-213, ISSN: 09240136

Abe, F., Santos, E. C., Kitamura, Y., Osakada K. and Shiomi M. (2003), Influence of forming conditions on the titanium model in rapid prototyping with the selective laser melting process, Journal of Mechanical Engineering Science, Vol. 217, pp.119-126, ISSN: 0022-2542

Agarwala, M., Bourell, D., Beaman, J., Marcus H. and Barlow J. (1995), Direct Selective Laser Sintering of Metals, Rapid Prototyping Journal, Vol. 1, Issue 1, pp. 26-36, ISSN: $1355-2546$

Akhtar, S., Wright, C. S., Youseffi, M., Hauser, C., Childs, T. H. C., Taylor, C. M., Badrossomay, M., Xie, J., Fox, P. and O’Neill, W. (2003), Direct SLS of Tool Steel Powders to High Density. Part B - the Effect on Microstructural Evolution, In Proceedings of the 14th Annual International Solid Freeform Fabrication Symposium, , Austin, Texas, USA, 4-6 August, pp. 656-667

Anatomics (2009), AnatomicsRX $\quad$ Software, $\quad$ [WWW] http://www.anatomics.com/content/default.aspx?cat $=2=23$ [Accessed on $22^{\text {nd }}$ April 2009]

Ang, L. K., Lau, Y. Y., Gilgenbach, R. M., and Spindler, H. L. (1997), Analysis of Laser Absorption on a Rough Metal Surface, Applied physics Letters, Vol. 70, Issue 6, pp. 696-698, ISSN: 0003-6951

Anusavice, K. J. (2003), Phillips' science of dental materials, W.B. Saunders, ISBN: 07216-9387-3

Arcam AB (2009), [WWW] http://www.arcam.com [Accessed on $12^{\text {th }}$ March 2009]

ARGEN $^{\odot}$ (2009), Alloy Makers to the World, [WWW] http://www.argen.com/, [Accessed on $13^{\text {th }}$ September 2009] 
ASTM B329 (1998), Standard Test Method for Apparent Density of Free Flowing Metal Powders using the Scott Volumeter, American Society for Testing Materials

ASTM B212 (1999), Standard Test Method for Apparent Density of Free Flowing Metal Powders using the Hall Flowmeter Funnel, American Society for Testing Materials, re-approved 2006

ASTM B527 (2006), Standard Test Method for Determination of Tap Density of Metallic Powders and Compounds, American Society for Testing Materials

Beer, R. T. and Ring, E. M. (1972), Liquid metals: chemistry and physics, New York, Marcel Dekker, ISBN: 0824710320

BEGO (2009), BEGO Medifacturing, [WWW] http://www.bego-medical.de/

Boivie, K. (2001), Limits of Loose Metal Powder Density in the Sinterstation, $12^{\text {th }}$ Annual Solid Freeform Fabrication Symposium, 6-8 August, Austin, Texas, USA

Bonch-Bruevich, A. M., Imas, Y. A., Romanov, G. S., M. N. Libenson, M. N. and Mal'tsev, L. N. (1968), Soviet Physics-Technical Physics, Vol. 13, pp.640, ISSN: 0038-5662

Brick, E. M., Rudolph. H., Arnold, J. and Luthardt. R. G. (2004), Analysis of threedimensional sinter shrinkage of copings made from alumina in an innovative direct shaping process, Journal of Computerized Medical Imaging and Graphics, Vol. 28, Issue 3, pp. 159-165, ISSN: 0895-6111

Brussel. V. K. (2002), Medical Image Based Personalised Drill Guides for Spinal Screw Insertion, Katholieke Universiteit Leuven, Belgium, Phd Thesis

BS EN 23923-1 (1993), Metallic Powders - Determination of Apparent Density-Part 1: Funnel Method, British Standard

BS EN 23923-2 (1993), Metallic Powders — Determination of Apparent Density - Part 2: Scott Volumeter Method, British Standard

BS EN ISO 3953 (1995), Metallic Powders - Determination of Tap Density, British Standard

Cascone, P. (2003), A Unique Gold Casting Alloy for Dental Applications, $3^{\text {rd }}$ International Conference on Gold Science and Applications, $28^{\text {th }}$ Sept $-1^{\text {st }}$ Oct, Vancouver, Canada

CEREC (2009), The CEREC Acquisition Centre Powered by Bluecam CAD/CAM for everyone, [WWW] http://www.cereconline.com/cerec/acquisition-center.html

Chandrasekhar, S. (1970), Hydrodynamic and Hydromagnetic Stability, Clarendon Press, Oxford, ISBN: 048664071X

Chelule, K. L., Coole, T. J. and Cheshire. D. G. (2000), Fabrication of Medical Models from Scan Data via Rapid Prototyping Techniques, In the Proceedings of TCT (Time-Compression Technologies) Conference and Exhibition, pp. 45-50, Cardiff, UK 
Cheng, J. and Kar, A. (1997), "Mathematical Model for Laser Densification of Ceramic Coating" Journal of Materials Science, Vol. 32, pp. 6269-6278

Childs, T. H. C. (2005), Raster scan selective laser melting of the surface layer of a tool steel powder bed, Proceedings of the Institution of Mechanical Engineers Part B: Journal of Engineering Manufacture, Vol. 219, Issue 4, pp. 379-384, ISSN: 09544054

Cormier, D., West H., Harrysson O. and Knowlson K. (2004), Characterization of Thin Walled Ti-6Al-4V Components Reduced via Electron Beam Welding, $15^{\text {th }}$ Annual Solid Freeform Fabrication Symposium, 2-4 August, Austin, Texas, USA

Coupland, J. (2009), Discussion with professor Jeremy Coupland, Professor of Applied Optics, Optical Engineering Research Group, Wolfson School of Mechanical and Manufacturing Engineering, Loughborough University, UK

Das, S. (1998), Direct Selective Laser Sintering of High Performance Metals - Machine Design, Process Development and Process Control, University of Texas at Austin, USA, Phd thesis

Daves, C. (1991), Laser Welding, New York, McGraw-Hill, Inc., ISBN: 0-07-016123-2

Deckers, J. (2008), Experimental investigation of Laser Surface Remelting for the Improvement of the Selective Laser Melting Process, The Proceedings of $19^{\text {th }}$ International Solid Freeform Fabrication Symposium, August 4-6, Texas, USA

Donnelly, R. J. and Glaberson, W. (1965), Experiments on the capillary instability of a liquid jet, Proceedings of the Royal Society of London, A290, pp. 547-556.

Duley, W. (1999), Laser Welding, Wiley, ISBN: 0-471-246679-4

Edelmann O. and Mueller-Lohmeier, K. (2005), Direct Prototyping of Parts in Light Metal Alloys and Steel by Laser Melting, In Rapid Prototyping \& Manufacturing Conference \& Exposition (SME), Dearborn, Michigan, USA, 10-12 May.

Eustathopoulos, N., Nicholas M. G. and Drevet B. (1999), Wettability at High Temperatures, Pergamon, ISBN: 0-08-042146-6

Extrand, C. W. (2003), A thermodynamic Model for Wetting Free Energies from Contact Angles, Langmuir, Vol. 19, Issue 3, pp. 646-649, ISSN: 0743-7463

Feenstra, F., Holmer B., Pohl H. G., Tromans, M. N. and Mieritz B. (2003), RP, RT, RM Trends and Developments/Research Survey, In the Proceeding 4th National Conference of Rapid \& Virtual Prototyping and Applications, pp. 95-138, Buckinghamshire, UK

Fischer-Cripps, A. C. (2006), Critical Review of Analysis and Interpretation of Nanoindentation Test Data, Surface and Coating Technology, Vol. 200, Issue 14-15, pp. 4153-4165,ISSN: 0257-8972

Fischer, P., Karapatis, N., Romano, V., Glardon, R. and Weber, H. P. (2002), A Model for the Interaction of Near-Infrared Laser Pulses with Metal Powders in Selective Laser Sintering, Applied Physics A, Vol. 74, Issue 4, pp. 467-474, ISSN: 0947-8396 
Fischer, P., Romano, V., Blatter A. and Weber H. P. (2005), Highly Precise Pulsed Selective Laser Sintering of Metallic Powders, Laser Physics Letters, Vol. 2, Issue 1, pp.48-55, ISSN: 1612-2011

Fischer, P., Romano, V., Weber, H. P., Karapatis, N. P., Boillat E. and Glardon R. (2003), Sintering of Commercially Pure Titanium Powder with A Nd:YAG Laser Source, Acta Materialia, Vol. 51, Issue 6, pp. 1651-1662, ISSN: 1359-6454

Fuh, J. Y. H., Y. S. Choo, A. Y. C. Nee, L. Lu and K. Lee (1995), Improvement of the V curing process for the laser lithography technique, Materials \& Design, Vol. 16, Issue 1, pp.23-32, ISSN: 0261-3069

GC Corporation (2009), Advanced Technologies, [WWW] http://www.gcdental.co.jp/english/pandt/tech.html

Geldart, D., \& Wong, C. Y. (1984). Fluidization of powders showing degrees of cohesiveness. 1. Bed expansion. Chemical Engineering Science, Vol. 39, pp. 14811488, ISSN: 0009-2509

German, R. M. (1989), Particle Packing Characteristics, Metal Powder Industry, Princeton, New Jersey, ISBN: 0-918404-83-5

Gibson, I. (2005), Advanced Manufacturing Technology for Medical Applications, Wiley, ISBN 0-470-01688-4

Gower, H. L., Pieters R. R. G. M. and Richardson I. M. (2005), Pulsed Laser Welding of Metal-Polymer Sandwich Materials using Pulse Shaping, Journal of Laser Applications, Vol. 18, Issue 1, pp.35-41, ISSN: 1042-346X

Grevey, D., Sallamand, P., Cicala E. and Ignat, S. (2005), Gas Protection Optimization during Nd:YAG Laser Welding, Optics and laser technology, Vol. 37, Issue 8, pp. 647-651, ISSN: 0030-3992

Gu, D. and Shen, Y. (2007), Balling Phenomena During Direct Laser Sintering of MultiComponent Cu-Based Metal Powder, Journal of Alloys and Compounds, Vol. 432, Issue 1-2, pp.163-166, ISSN: 0925-8388

Gusarov, A. V., Yadroitsev, I., Bertrand, Ph. and Smurov, I. (2007), "Heat Transfer Modelling and Stability Analysis of Selective Laser Melting" , Applied Surface Science, Vol. 254, pp. 975-979

Harnby, N., Hawkins, A. E. and Vandame, D. (1987), The Use of Bulk Density Determination as a Mean of Typifying the Flow Characteristics of Loosely Compacted Powders Under Conditions of Variable Relative Humidity, Chemical Engineering Science, Vol. 42, pp. 879-888, ISSN: 0009-2509

Hauser, C., Sutcliffe, C., Egan M. and Fox P. (2005), Spiral Growth Manufacturing (SGM) - A Continuous Additive Manufacturing Technology For Processing Metal Powder by Selective Laser Melting, $19^{\text {th }}$ Annual International Solid Freeform Fabrication Symposium, 4-6 August, Austin, Texas, USA 
Hausner, H. H. (1967), Friction Conditions in a Mass of Metal Powder, International Journal of Powder Metallurgy, Vol. 3, Issue 4, pp. 7-13, ISSN: 0888-7462

Hegge, H. J. and Hosson, J. (1991), The influence of convection on the homogeneity of laser-applied coatings, Journal of Material Science, Vol. 26, pp. 711-714

Hsiao, Y. C., Shimizu, H., Firth, L., Maher, W. and Masubuchi, K. (1997), "Finite Element Modeling of Laser Forming", Proceeding of Laser Materials Processing Conference ICALEO'97, San Diego, CA, USA, Vol. 83, pp. 31-40

Ion, J. C. (2005), Laser Processing of Engineering Materials, Butterworth-Heinemann, ISBN: 0750660791

ISO 3923-1 (1979), Metallic Powders - Determination of Apparent Density - Part 1: Funnel Method, International Organization for Standardization

ISO 3923-2 (1981), Metallic Powders - Determination of Apparent Density - Part 2: Scott Volumeter Method, International Organization for Standardization

Jeng, J. Y. and Lin M. C. (2003), Mold Fabrication and Modification using Hybrid Processes of Selective Laser Cladding and Milling, Journal of Materials Processing Technology, Vol. 1, Issue 110, pp.98-103, ISSN: 0924-0136

Karapatis, N. P. (2002), A Sub-Process Approach of Selective Laser Sintering, Ecole Polytechnique Federal de Lausanne (EPFL), Switzerland, Phd Thesis

Karapatis, N. P., Griethuysen J. P. S. and Glardon R. (1998), Direct Rapid Tooling: A Review of Current Research, Rapid Prototyping Journal, Vol. 4, Issue 2, pp. 77-89, ISSN: $1355-2546$

Kong, C. Y., Soar, R. C. and Dickens, P. M. (2003), Optimum Processing Parameters for Ultrasonic Consolidation of 3003 Aluminium, Journal of Material Processing Technology, Vol. 146, Issue 2, pp. 181-187, ISSN: 0924-0136

Kou, S. (2003), Welding Metallurgy, Wiley Interscience, ISBN: 0-471-43491-4

Kruth, J. P. (1991), Material Increases Manufacturing by Rapid Prototyping Techniques, CIRP Annals- Manufacturing Technology, Vol. 40, Issue 2, pp. 603-614, ISSN: 0007-8506

Kruth, J. P., Froyen, L., Rombouts, M., Vaerenbergh J. V. and Mercelis P. (2003), New Ferro Powder for Selective Laser Sintering of Dense Parts, CIRP Annals Manufacturing Technology, Vol. 1, Issue 52, pp. 139-142, ISSN: 0007-8506

Kruth, J. P., Froyen, L., Vaerenbergh, J. V., Mercelis, P., Rombouts M. and Lauwers B. (2004), Selective Laser Melting of Iron-Based Powder, Journal of Materials Processing Technology, Vol. 149, Issue 1-3, pp. 616-622, ISSN: 0924-0136

Kruth, J. P., Levy, G., Klocke, F. and Childs T. H. C. (2007), Consolidation Phenomena in Laser and Powder-Bed Based Layered Manufacturing, CIRP Annals - Manufacturing Technology, Vol. 56, Issue 2, pp. 730-759, ISSN: 0007-8506 
Kruth, J. P., Mercelis, P., Vaerenbergh, J. V., Froyen L. and Rombouts, M. (2005), Binding Mechanisms in Selective Laser Sintering and Selective Laser Melting, Rapid Prototyping Journal, Vol. 11, Issue 1, pp. 26-36, ISSN: 1355-2546

Laeng, J., Stewart J. G. and Liou F. W. (2000), Laser Metal Forming Processes for Rapid Prototyping - a review, International Journal of Production Research, Vol. 38, Issue 16, pp. 3973-3996, ISSN: 0020-7543

Laserdenta

(2009),

[WWW] http://www.laserdenta.com/Product_details/Laserdenta_Dental_Scanner.html [Accessed on $22^{\text {nd }}$ June 2009]

Lawrence, J., Peligrad, A. A., Zhou, E., Li, L. and Morton, D. (2001), "Prediction of Melt Depth in Selected Architectural Materials During High-Power Diode Laser Treatment" Optical Lasers Engineering, Vol. 35, pp. 51-62

Leu M. C. and Delli. P. (2008), Virtual Prototyping and Bio-Manufacturing in Medical Applications, Springer, ISBN 978-0-387-33429-5

Leung, M. (2001), Phase-Change Heat Transfer in Laser Transformation Hardening by Moving Gaussian Rectangular Heat Source, Journal of Physics D, Vol. 34, pp. 34343441

Li, J. F., Li, L. and Stott, F. H. (2004a), "Thermal Stresses and their Implication on Cracking During Laser Melting of Ceramic Materials" Acta Materialia, Vol. 52, pp. 4385-4398

Li, J. F., Li, L. and Stott, F. H. (2004b) "A Three-Dimensional Numerical Model for a Convection-Diffusion phase Change Process During Laser Melting of Ceramic Materials" International Journal of Heat and Mass Transfer, Vol. 47, pp. 5523-5539

Li, J. F., Li, L. and Stott, F. H. (2004c), "Comparison of Volumetric and Surface Heating Sources in the Modelling of Laser Melting of Ceramic Materials", International Journal of Heat and Mass Transfer, Vol. 47, pp. 1159-1174

Lu, L., Fuh J. and Wong Y. S. (2001), Laser-Induced Materials and Processes for Rapid Prototyping, Kluwer Academic Publishers, USA, ISBN: 0-7923-7400-2

Lu, S., Fuji, H. and Nogi, K. (2004), Marangoni convection and weld shape variations in Ar-O2 and Ar-CO2 shielded GTA welding. Materials Science and Engineering A, Vol. 380, Issue 1-2, pp. 290-297

Luthardt, R. G., Sandkuhl, O. and Reiz. B., (1999), Zirkonia-TZP and Alumina - Advanced Technologies for the Manufacturing of Single Crowns, European Journal of Prosthodontics and Restorative Dentistry, Vol. 7, pp. 113, ISSN: 0965-7452

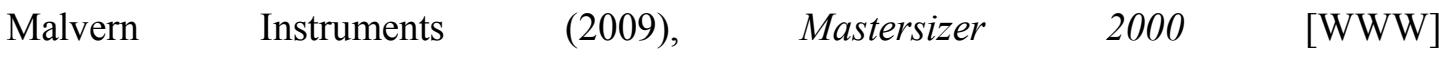
http://www.malvern.com/LabEng/products/Mastersizer/MS2000/mastersizer2000.ht $\mathrm{m}$ [Accessed on $3^{\text {rd }}$ April 2009] 
Materialise (2009), Rapid Shell Modelling (RMS), [WWW] http://www.materialise.com/materialise/view/en/92102-Rapid+Shell+Modelling+ (RSM).html

Matsumoto, M., Shiomi, M., Osakada, K. and Abe, F. (2002), "Finite element analysis of single layer forming on metallic powder bed in rapid prototyping by selective laser processing", International Journal of Machine Tool and Manufacture, Vol. 42, pp. 6167

Mazumder, J., Dutta, D., Kikuchi N. and Ghosh A. (2000), Closed Loop Direct Metal Deposition: Art to Part, Optics and Lasers in Engineering, Vol. 34, Issue 4-6, pp. 397-414, ISSN: 0143-8166

Mc Gurk, M., Aimis, A. A., Potamianos, P. and Goodger. N. M. (1997), Rapid Prototyping Techniques for Anatomical Modelling in Medicine, Annual Royal College of Surgery of England, Vol. 79, pp. 167-174, ISSN: 0035-8843

MCP-HEK GmbH (2009), [WWW] http://www.mcp-group.de/.

Messler, R. W. (1999), Principles of Welding, Processes, Physics, Chemistry and Metallurgy, Wiley - Interscience, ISBN: 0-471-25376-6

Mills, C., Keene B. J. and Brooks K. (1998), Marangoni Effects in Welding, Philosophical Transactions of the Royal Society A: Mathematical, Physical and Engineering Sciences, Vol. 356, Issue 1739, pp. 911-925, ISSN: 1471-2962

Morgan, R., Sutcliffe C. J. and O'Neill W. (2004), Density Analysis of Direct Metal Laser Re-Melted 316L Stainless Steel Cubic Primitives, Journal of Materials Science, Vol. 39, Issue 4, pp.1195-1205, ISSN: 0022-2461

Mormann. W. H. (2006), The Evolution of the Cerec System, Journal of the American Dental Association, Vol. 137 (9 supplement), pp. 7S-13S, ISSN: 0002-8177

Mumtaz, K. A. (2008), Selective Laser Melting of Inconel 625 Using Pulse Shaping, Loughborough University, UK, $\mathrm{PhD}$ thesis

Mumtaz, K. A., Erasenthiran, P. and Hopkinson, N. (2008), High Density Selective Laser Melting of Waspaloy ${ }^{\circledR}$, Journal of Materials Processing Technology, Vol. 195, Issue 1-3, pp 77-87, ISSN: 0924-0136

Mumtaz, K. A., Hopkinson N. and Erasenthiran P. (2006), High Density Selective Laser Melting of Waspaloy, Journal of Materials Processing Technology, Vol. 195, Issue 13, pp. 77-87, ISSN: 0924-0136

Niu, H. J. and Chang, I. T. H. (1999), Selective Laser Sintering of Gas and Water Atomized High Speed Steel Powders, Scripta Materialia, Vol. 41, Issue 1, pp. 25-30, ISSN: $1359-6462$

Nobel Biocare (2009), [WWW] http://www1.nobelbiocare.com/en/implants-andabutments/home/default.aspx [Accessed June 2009]

Odumodu, K. U. and Das, S. (1996), "Forceless Forming with Laser", Proceeding of ASME Int, Vol. 74, pp. 169-170 
Ohmura, E., Hayashi, H., Fujimori S. and Miyamoto. I. (1997), Thermohydrodynamics analysis of fusion phenomena accompanied with evaporation due to laser irradiation, Proceedings of the Laser Institute of America (ICALEO), CA, USA, pp. 9-15

Oliver, W. C. and Pharr, G. M. (2003), Measurement of Hardness and Elastic Modulus by Instrumented Indentation: Advances in Understanding and Refinements to Methodology, Journal of Materials Research, Vol. 19, Issue 1, pp.3-20, ISSN: 08842914

O'Neill, W., Sutcliffe, C. J., Morgan R. and Hon K. K. B. (1998), Investigation of Short Pulse Nd:YAG Laser Interaction with Stainless Steel Powder Beds, $9^{\text {th }}$ Solid Freeform Fabrication Symposium, 10-12 August, Austin, Texas, USA

Over, C., Meiners, W., Wissenbach, K., Lindemann, M. and Hammann, G. (2001), Selective Laser Melting: A New Approach for the Direct Manufacturing of Metal Parts and Tools, In Proceedings of the 3rd Laser Assisted Net Shape Engineering Conference (LANE2001), Erlangen, Germany, Bamberg-Meisenbach, 28-31 August, pp. 391-398

Park, H. and Rhee, S. (1999), Analysis of mechanism of plasma and spatter in CO2 laser welding of galvanized steel, Optics and Laser Technology, Vol. 31, Issue 2, pp. 119126, ISSN: 0030-3992

Photonics, R. (2009), Encyclopedia of Laser Physics and Technology [WWW] http://www.rp-photonics.com/fiber_lasers.html, [Accessed on January 2009]

Pinkerton, A. J., Karadge, M., Syed, W. and Li, L. (2006), Thermal and Microstructural Aspects of the Laser Direct Metal Deposition of Waspaloy, Journal of Laser Applications, Vol. 18, Issue 3, pp.216-226, ISSN: 1042-346X

Pogson, S., Fox, P., O'Neill, W. and Sutcliffe, C. J. (2004), The direct metal laser remelting of copper and tool steel powders, Materials Science and Engineering A, Vol. 386, Issue 1-2, pp. 453-459, ISSN: 0921-5093

Pohl, H., Petzoldt, F., Terada, M., Kakiuchi, E. and Kanazawa I. (2004), DMLS of Titanium Alloy, In Proceedings of PM2004 Conference, Vienna, Austria, 17-21 October, pp. 141-146, 2004.

Pohl, H., Simchi, A., Issa M. and Dias H. C. (2001), Thermal Stresses in Direct Metal Laser Sintering, $12^{\text {th }}$ Annual International Solid Freeform Fabrication Symposium, 68 August, Austin Texas, USA

Rayleigh, L. (1892), On the Instability of a Cylinder of Viscous Liquid under Capillary Force, Philosophical Magazine, Vol. 34, Issue. 207, pp. 145-154

Ready, J. F. (2001), LIA Handbook of Laser Materials Processing, Laser Institute of America, Magnolia Publishing Inc., ISBN: 0-941463-02-8

Renishaw (2009), Incise $e^{T M}$ Scanner and CAD Software, [WWW] $\mathrm{http}: / / \mathrm{www} \cdot$ renishaw.com/en/10249.aspx [Accessed on $12^{\text {th }}$ June 2009] 
Roberts, I. A., Wang, C. J., Esterlein, R., Stanford, M. and Mynors D. J. (2009), “A threedimensional finite element analysis of the temperature field during laser melting of metal powders in additive layer manufacturing", International Journal of Machine Tool and Manufacture, Vol. 49, pp. 916-923

Rombouts, M. (2006), Selective Laser Sintering/Melting of Iron Based Powders, Katholieke Universiteit Leuven, Belgium, Phd Thesis

Rombouts, M., Kruth, J. P., Froyen L. and Mercelis P. (2006), Fundamentals of Selective Laser Melting of alloyed steel powders, CIRP Annals - Manufacturing Technology, Vol. 55, Issue 1, pp. 187-192, ISSN: 0007-8506

Rossi, S., Deflorian, F. and Venturini, F. (2004), Improvement of Surface Finishing and Corrosion Resistance of Prototypes Produced by Direct Metal Laser Sintering, Journal of Materials Processing Technology, Vol. 148, Issue 3, pp. 301-309, ISSN: 0924-0136

Santomaso, A., Lazzaro, P. and Canu, P. (2003), Powder Flowability and Density Ratios: The Impact of Granules Packing, Chemical Engineering Science, Vol. 58, Issue 13, pp. 2857-2874, ISSN: 0009-2509

Santos, E. C., Osakada, K., Shiomi, M., Kitamura, Y. and Abe, F. (2004), Microstructure and mechanical properties of pure titanium models fabricated by selective laser melting, Journal of Mechanical Engineering Science, Vol. 218, Issue 7, pp. 711-719, ISSN: 0022-2542

Santos, E. C., Shiomi, M., Osakada K. and Laoui T. (2006), Rapid Manufacturing of Metal Components by Laser Forming, International Journal of Machine Tools and Manufacture, Vol. 46, Issue 12-13, pp.1459-1468, ISSN: 0890-6955

Scott, G. D. (1960), Packing of equal spheres, Nature, Vol. 188, pp. 908-909, ISSN: 0028-0836

Sears, J. W. (1999), Direct Laser Powder Deposition - 'State of the Art'. ASTM/TMS Materials Week, Cincinnati, Ohio,US,16 pages

Semak, V. and Matsunawa, A. (1997), The Role of Recoil Pressure in Energy Balance During Laser Materials Processing, Journal of Physics D: Applied Physics, Vol. 30, Issue 18, pp. 2541-2552, ISSN: 0022-3727

Sexton, L., Lavin, S., Byrne G. and Kennedy A. (2002), Laser Cladding of Aerospace Materials, Journal of Materials Processing Technology, Vol. 122, Issue 1, pp. 63-68, ISSN: 0924-0136

Shannon, G. J. and Li B. Q. (2004), Investigation of Weld Cracking in Nd:YttriumAluminum-Garnet Keyhole Spot Welding of Materials used in Optoelectronic Packaging, Journal of Laser Applications, Vol. 16, Issue 2, pp. 73-78, ISSN: $1042-$ $346 \mathrm{X}$

Shishkovsky I., Yadroitsev I., Bertrand P. and Smurov I. (2007), Alumina-Zirconium Ceramics Synthesis by Selective Laser Sintering/ Melting, Applied Surface Science, Vol. 254, Issue 4, pp. 966-970, ISSN: 0169-4332 
Simchi, A. (2004), The Role of Particle Size on the Laser Sintering of Iron Powder, Metallurgical and Materials Transactions B, Process Metallurgy and materials Processing Science, Vol. 35, Issue 5, pp. 937-948, ISSN: 1073-5615

Simchi, A. (2006), Direct Laser Sintering of Metal Powders: Mechanism, Kinetics and Microstructural Features, Materials Science and Engineering: A, Vol. 428, Issue 1-2, pp. 148-158, ISSN: 0921-5093

Simpleware (2009), ScanIP Software, [WWW] http://www.simpleware. com/software/scanip.php

Singheiser, L., Steinbrech, R., Quadakkers W. J. and Herzog R. (2001), Failure Aspects of Thermal Barrier Coatings, Materials at High Temperatures, Vol. 18, Issue 4, pp. 249-259, ISSN: 0960-3409

Sirona (2009), [WWW] http://www.sirona.com/ecomaXL/index.php?site=sirona_e_ho me

Steen, W. M. (2003), Laser Material Processing, $3^{\text {rd }}$ ed., Springer-Verlag London Ltd., ISBN: 1-85233-698-6

Strietzel, R. (2004), Production of Metallic Frames Using the BEGO Medifacturing Process, Dental Technology, Vol. 50, pp. 37-44, ISSN: 1088-3118

Su, W. N. (2002), Layered Fabrication of Tool Steel and Functionally Graded Materal with a Nd:YAG Pulsed Laser, PhD Thesis, Loughborough University. UK

Svarovsky, L. (1987), Powder Testing Guide: Methods of Measuring the Physical Properties of Bulk Powders, Elsevier Applied Science Publishers Ltd., London and New York, ISBN: 1-85166-137-9

Swaelens, B. and Kruth, J. P. (1993), Medical applications of Rapid Prototyping Techniques, In Proceedings of the 4th International Conference on Rapid Prototyping, pp. 107-120

Syvanen, T., Nyrhila, J., Kotila J. and Lind J. E. (2000), Direct Metal Laser Sintering of Very Fine Metal Powder, In Proceddings of $19^{\text {th }}$ International Congress on Applications of Lasers and Electro-Optics (ICALEO), 2-5 October, Dearborn, Vol. 89, Section D 21-29, Michigan, USA

Thorsson, L. (2006), Laser Sintering Gold Jewellery, In the Proceeding of $1^{\text {st }}$ International Conference on Rapid Manufacturing, 5-6 July, pp. 7-19 Loughborough University, UK

Tolochko, N. K., Khlopkov, Y. V., Mozzharov, S. E., Sobolenko, N. V. and Yadroitsev, I. A. (1997), Measurement Of The Absorption Coefficient Of Freely Poured OneComponent Metallic Powders And Its Change During Laser Sintering, Powder Metallurgy and Metal Ceramics, Vol. 36, Issue 7-8, pp. 433-437, ISSN: 1068-1302

UTHSCSA. (2002), UTHSCSA Image Tool Version 3.0 Final, [WWW] http://ddsdx.uthscsa.edu/dig/itdesc.html, [Accessed on 12 ${ }^{\text {th }}$ March 2009] 
Vandenbroucke, B. (2008), Optimizing and Characterizing the Selective Laser Melting (SLM) Process for Biocompatible Metals, Katholieke Universiteit Leuven, Belgium, Phd Thesis

Van Der Schueren, B. (1996), Basic Contributions to the Development of the Selective Metal Powder Sintering Process, University of Leuven, Belgium, PhD thesis

Van Elsen, M. (2007), Complexity of Selective Laser Melting: A New Optimisation Approach, Katholieke Universiteit Leuven, Belgium, Phd Thesis

WIELAND Dental (2009), ZENOTEC 1, [WWW] http://www.wielanddental.de/startseite/page.html? L $=1$ [Accessed on $5^{\text {th }}$ June 2009]

Williams C.B, Mistree F. and Rosen D.W (2005), Towards the Design of Layer-Based Additive Manufacturing Process for the Realization of Metal Parts of Designed Microstructure, In Proceedings of the $16^{\text {th }}$ Solid Freeform Fabrication Symposium, 1 3 August, Austin, Texas, USA

Wohlers, T. (2008), Wohlers Report 2008, Rapid Prototyping and Manufacturing State of the Industry, ISBN: 0-9754429-3-7

Wong, A. C. (2000), Characterisation of the flowability of glass beads by bulk densities ratio, Chemical Engineering Science, Vol. 55, pp. 3855-3859, ISSN: 0009-2509

Xie, J. and Kar A. (1999), Laser Welding of Thin Sheet Steel with Surface Oxidation, Welding Journal- Welding Research Supplement, Vol. 78, Issue 10, pp. 343-348, ISSN: 0043-2296

Yadroitsev, I., Thivillon, L., Bertrand, P. and Smurov, I. (2007), Strategy of manufacturing components with designed internal structure by selective laser melting of metallic powder, Applied Surface Science, Vol. 254, Issue 4, pp. 980-983, ISSN: 0169-4332

Yadroitsev, I. , Shishkovsky, I., Bertrand, P. and Smurov, I. (2009), Manufacturing of Fine-Structured 3D Porous Filter Elements by Selective Laser Melting, Journal of Applied Surface Science, Vol. 255, Issue 10, pp. 5523-5527, ISSN: 0169-4332

Yagla, J. J., Haag, R. S. and Scott, M. E. (1995), "Laser welding analysis and experiments", Journal of Ship Production, Vol. 11, pp. 102-110

Yakovlev, A., Trunova, E., Grevey, D., Pilloz M. and Smurov I. (2005), Laser-Assisted Direct Manufacturing of Functionally Graded 3D Objects, Surface and Coatings Technology, Vol.190, Issue 1, pp.15-24, ISSN: 0257-8972

Yang, L. X. , Peng, X. F. and Wang, B. X., (2001) "Numerical Modelling and Experimental Investigation on the Characteristics of Molten Pool During Laser Processing", International Journal of Heat and Mass Transfer, Vol. 44, pp. 4465-4473

Yevko, V., C. B. Park, G. Zak, T. W. Coyle and B. Benhabib (1998), Cladding Formation in Laser-Beam Fusion of Metal Powder, Rapid Prototyping Journal, Vol. 4, Issues 4, pp. 168-184, ISSN: 1355-2546 
Yu, A. B. and Hall, J. S. (1994), Packing of Fine Powders Subjected to Tapping, Powder Technology, Vol. 78, pp. 247-256, ISSN: 0032-5910

Zhang, G., Wei, Z. and Farrell, R. E. (2009), Elastic Modulus and Hardness of Muscovite and Rectorite Determined by Nanoindentation, Applied Clay Science, Vol. 43, pp. 271-281, ISSN: 0169-1317

Zhong, M., Sun, H., Liu, W., Zhu X. and He J. (2005), Boundary Liquation and Interface Cracking Characterization in Laser Deposition of Inconel 738 on Directionally Solidified Ni-Based Superalloy, Scripta Materialia, Vol. 53, Issue 2, pp. 159-164, ISSN: 1359-6462

Zhu, H. H., Fuh, J. Y. H. and Lu, L. (2007), The Influence of Powder Apparent Density on the Density in Direct Laser-Sintered Metallic Parts, International Journal of Machine Tools and Manufacture, Vol.47, Issue 2, pp 294-298, ISSN: 0890-6955

Zou, R. P. and Yu, A. B. (1995), Evaluation of the packing characteristics of mono-sized non-spherical particles, Powder Technology, Vol. 88, pp. 71-79, ISSN: 0032-5910 


\section{APPENDICES}

\section{Particle Size Distribution Analysis in Water}

Appendix-A

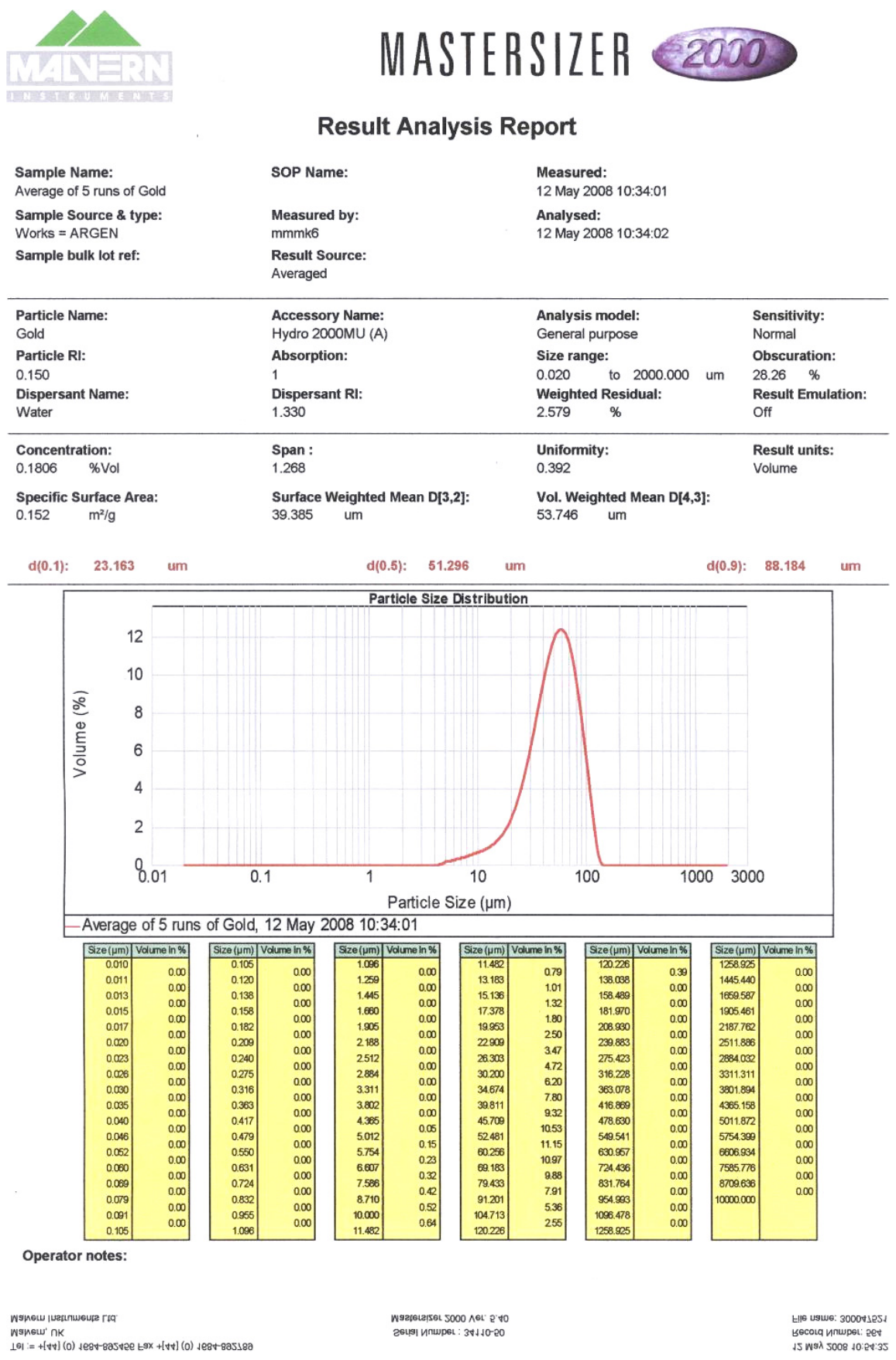




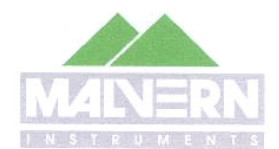

Sample Name:

Average of 5 runs of Gold

Sample Source \& type:

Works $=$ ARGEN

Sample bulk lot ref:

\section{MASTERSIZER 2000}

\section{Result Analysis Report}

$\begin{array}{ll}\text { SOP Name: } & \text { Measured: } \\ & 12 \text { May 2008 10:34:01 } \\ \text { Measured by: } & \text { Analysed: } \\ \text { mmmk6 } & 12 \text { May 2008 10:34:02 } \\ \text { Result Source: } & \\ \text { Averaged } & \end{array}$

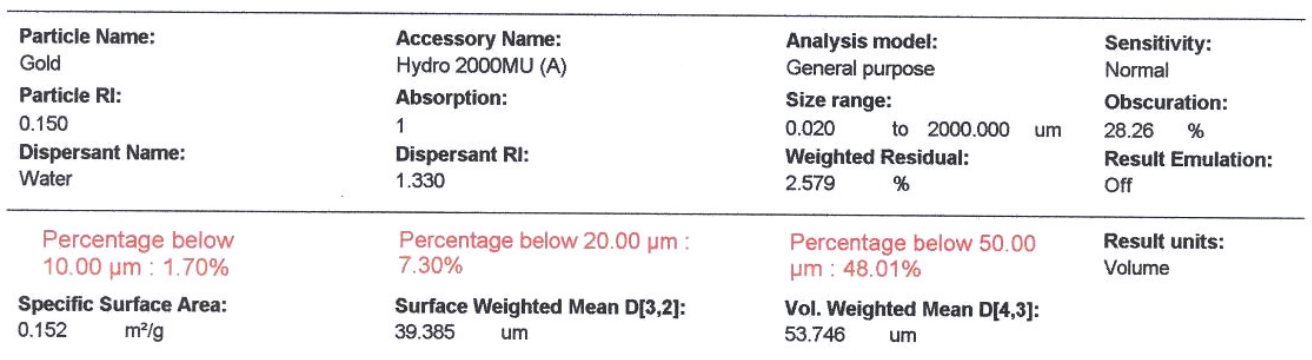

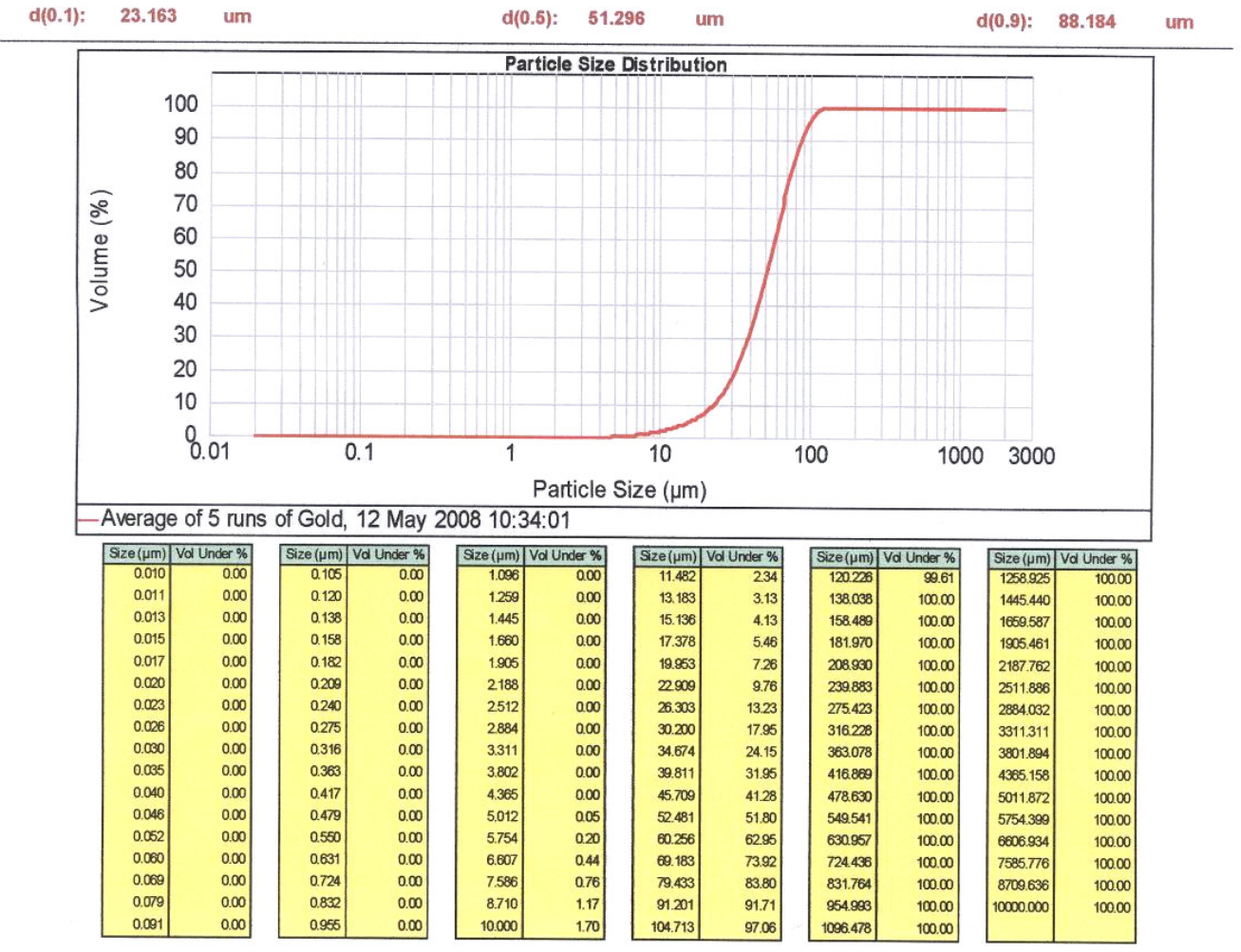

Operator notes:

WSMet fuaghnweupa rta

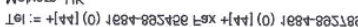

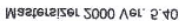

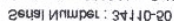

Hil uswe: 3000475s.

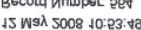




\section{Particle Size Distribution Analysis in Isopropanol}

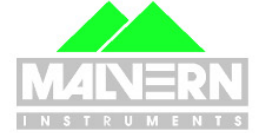

Sample Name:

Averaged Result_prop_1.5

Sample Source \& type:

Works $=$ ARGEN

Sample bulk lot ref:

\section{Particle Name:}

Gold

Particle RI:

0.150

Dispersant Name:

Propan-2-ol

Concentration:

$0.0373 \%$ Vol

Specific Surface Area:

$0.313 \quad \mathrm{~m}^{2} / \mathrm{g}$

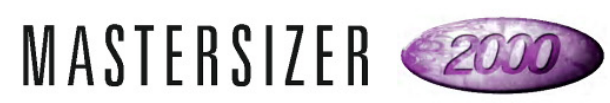

\section{Result Analysis Report}

SOP Name: $\quad$ Measured:

12 May 2008 12:05:47

Analysed:

Measured by:

mmmk6

Result Source:

Averaged

\section{Accessory Name:}

Hydro 2000MU (A)

Absorption:

1

Dispersant RI: 1.390

Span :

1.480

Surface Weighted Mean D[3,2]:

19.189 um
12 May 2008 12:05:48

\begin{tabular}{|c|c|c|}
\hline \multicolumn{2}{|c|}{$\begin{array}{l}\text { Analysis model: } \\
\text { General purpose }\end{array}$} & $\begin{array}{l}\text { Sensitivity: } \\
\text { Normal }\end{array}$ \\
\hline \multicolumn{2}{|c|}{ Size range: } & Obscuration: \\
\hline 0.020 & to $2000.000 \mathrm{um}$ & $13.28 \%$ \\
\hline \multicolumn{2}{|c|}{ Weighted Residual: } & \multirow{2}{*}{$\begin{array}{l}\text { Result Emulation: } \\
\text { Off }\end{array}$} \\
\hline .431 & $\%$ & \\
\hline
\end{tabular}

Vol. Weighted Mean D[4,3]:

26.815 um

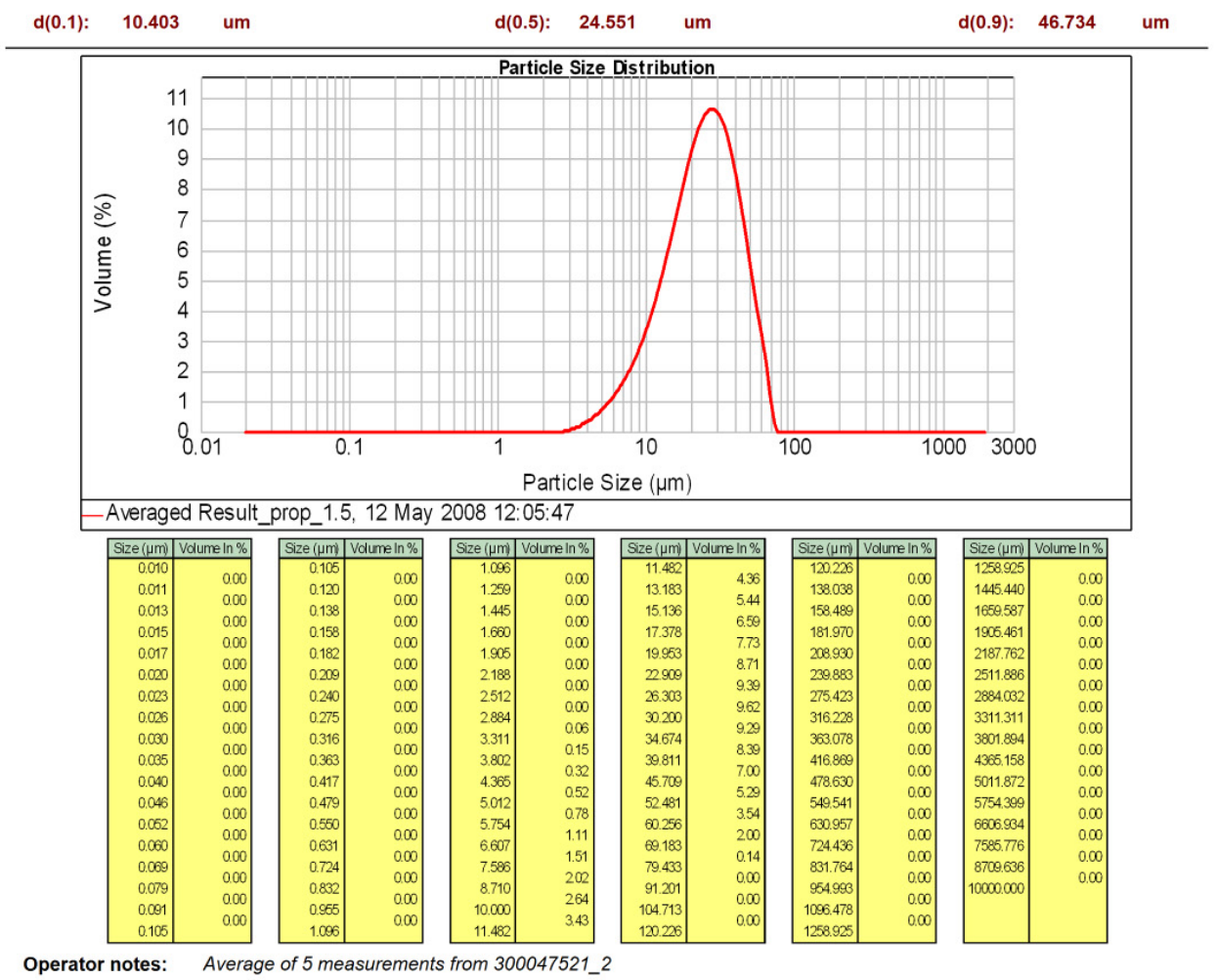




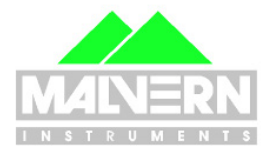

Sample Name:

Averaged Result_prop_1.5

Sample Source \& type:

Works $=$ ARGEN

Sample bulk lot ref:

\section{Result Analysis Report}

SOP Name: $\quad$ Measured:

12 May 2008 12:05:47

$\begin{array}{ll}\text { Measured by: } & \text { Analysed: } \\ \text { mmmk6 } & 12 \text { May } 2008 \text { 12:05:48 }\end{array}$

mmmk6

Result Source:

Averaged

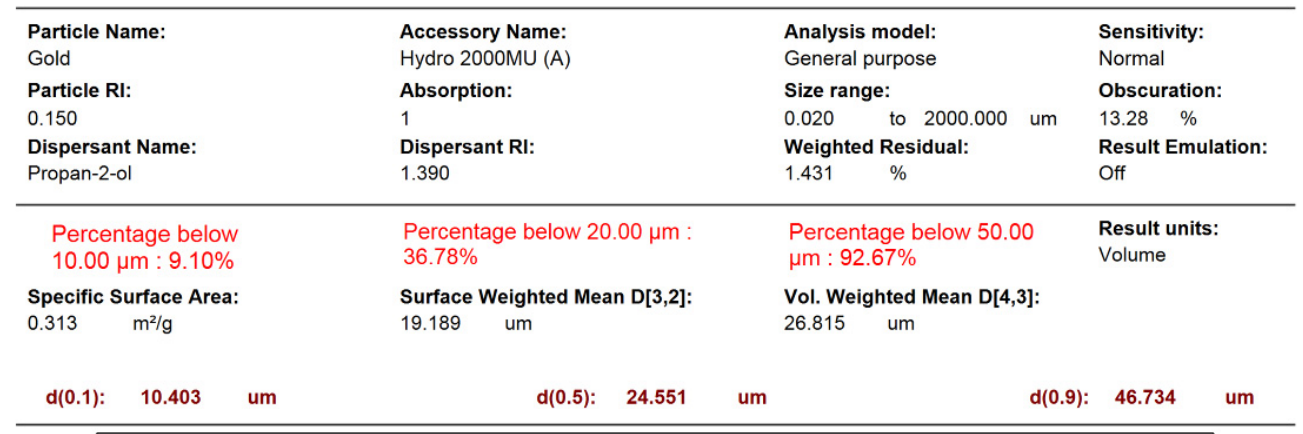

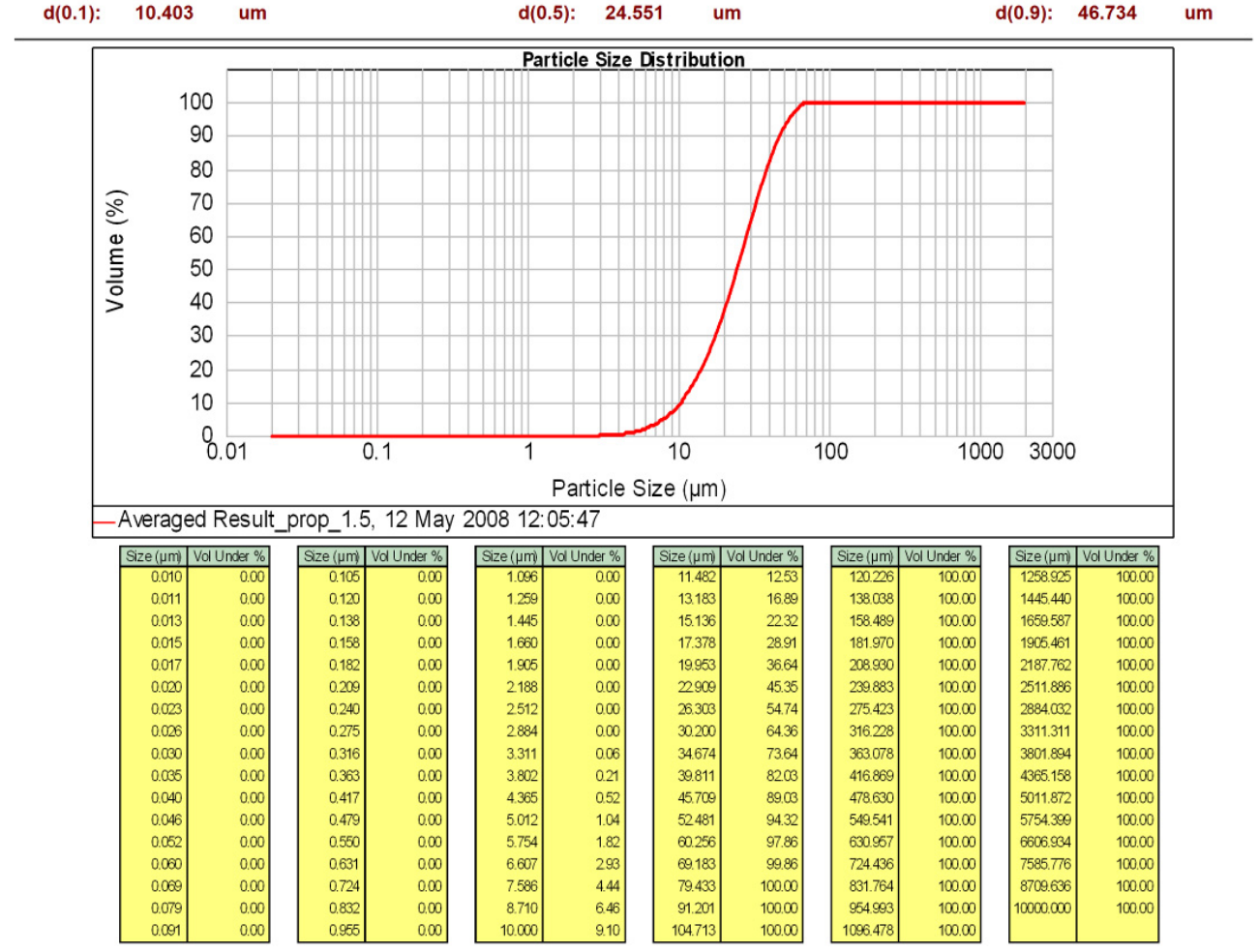

Operator notes: $\quad$ Average of 5 measurements from $300047521 \_2$ 


\title{
Appendix-B
}

\section{PROCESSING PARAMETERS FOR SELECTIVE LASER MELTING (SLM) OF GOLD}

\author{
M. Khan and P. M. Dickens \\ Rapid Manufacturing Research Group, Loughborough University \\ Loughborough, United Kingdom
}

\begin{abstract}
Research into laser processing of different metals has enabled Solid Freeform Fabrication (SFF) processes to produce parts for a wide variety of applications. However, less focus has been made on the processing of precious metals. Currently little research has been reported on the processing of precious metals and alloys using the Selective Laser Melting (SLM) process. Here we present an initial investigation into the processing of 24 carat gold $\mathrm{Au}$ ) powder using a SLM system. Gold powder was tested for apparent density, tap density, particle shape and size distribution. A quality check of the specimen was carried out using a Scanning Electron Microscope (SEM) for sinterability and occurrence of porosity. Significant processing parameters were also identified.
\end{abstract}

\section{INTRODUCTION}

Laser based Solid Freeform Fabrication (SFF) processes like selective laser sintering (SLS), Laser Engineered Net Shaping (LENS ${ }^{\mathrm{TM}}$ ), Direct Light Fabrication (DLF), Direct Metal Laser Sintering (DMLS) and Selective Laser Melting (SLM) [1-6] induce flexibility into the production of intricate Three-Dimensional (3D) complex metallic parts. All of these processes vary either in the type of laser source $\left(\mathrm{CO}_{2}\right.$, lamp or diode $\mathrm{Nd}$ :YAG and fiber or disc lasers etc.) used or type of diffusion (sintering or melting) occurring in the thin layers of metallic powder. Research into the laser processing of different metals and alloys (e.g. aluminium, nickel, copper alloy, titanium and tool steel etc) has enabled these rapid manufacturing systems to produce parts for a wide variety of applications such as automotive, aerospace and medical implants etc. Gu and Shen [7] used copper powder to investigate balling phenomena in the DMLS process, Mumtaz et al [8] processed nickel based super alloy (Waspaloy ${ }^{\circledR}$ ) using a high power pulse Nd:YAG laser system. Yadroitsev et. al. [9] processed Inconel 625 using Phenix System's PM 100 system. Simchi [10] sintered various ferrous powders: $316 \mathrm{~L}$ stainless steel powder and M2 powders by EOS M250X ${ }^{\text {tend }}$ machine. Rombouts et al [5] investigated laser melting of different size and mixture of water and gas atomized steel powders. Simchi [11] also investigated particle size effect on the sintering of iron powders. Fischer et al [12] sintered commercially pure titanium powder using an $\mathrm{Nd}$ :YAG laser. However, less focus has been made on the processing of precious metals and alloys. Thorsson [13] sintered 18 carat gold powder using EOS M250X ${ }^{\text {tend }}$ machine to fabricate jewellery items. Currently very little research has been reported on the processing of precious metals and alloys using the SLM process. Precious metals and alloys such as gold, silver, platinum and their alloys could be used for different applications like jewellery and dental implants etc. These parts could be easily produced using the manufacturing flexibility provided by the SFF processes. This investigation gives an initial insight into the processing of gold powder using the SLM system.

\section{MATERIAL CHARACTERISTICS}

The material used was 24 carat gold $(\mathrm{Au})$ powder.

\subsection{Particle Size Distribution}

Particle Size Distribution (PSD) of gold powder was carried out with the laser diffraction technique using a Malvern Mastersizer 2000. Gold powder was first dispersed in water but due to its poor dispersion, isopropanol was then used as the dispersing 
medium. The PSD results obtained using water as a dispersant showed the smallest particle size of around $5 \mu \mathrm{m}$ whereas smallest particle size obtained with isopropanol (Figure 1) was as low as $3.3 \mu \mathrm{m}$. For confirmation of the results, PSD was performed again using isopropanol, which also gave the same results. This difference in the two particle sizes was attributed to the fact that gold powder does not disperse well in water as compared to isopropanol. This was evident from the visual observation of both these solutions, where the colour of isopropanol changed to mild yellow on addition of gold powder, where no such observation was made in water and gold powder was floating on top. The SEM images (Figure 2a-d) obtained for gold powder also suggests smallest particles size of around $3 \mu \mathrm{m}$. Figure $2 \mathrm{c}$ (marked in circle) shows smaller satellite particles sticking to the larger particles forming agglomerates. When this agglomerated gold powder was added to water in the PSD analysis, it dispersed very little hence giving a maximum particle size of around $88 \mu \mathrm{m}$. The same gold powder when added to isopropanol for PSD analysis was dispersed well and indicated a maximum particle size of around $46 \mu \mathrm{m}$. The SEM images taken at different points in the gold sample did not show any particles of around $85 \mu \mathrm{m}$ but particles around $45 \mu \mathrm{m}$ were abundant.
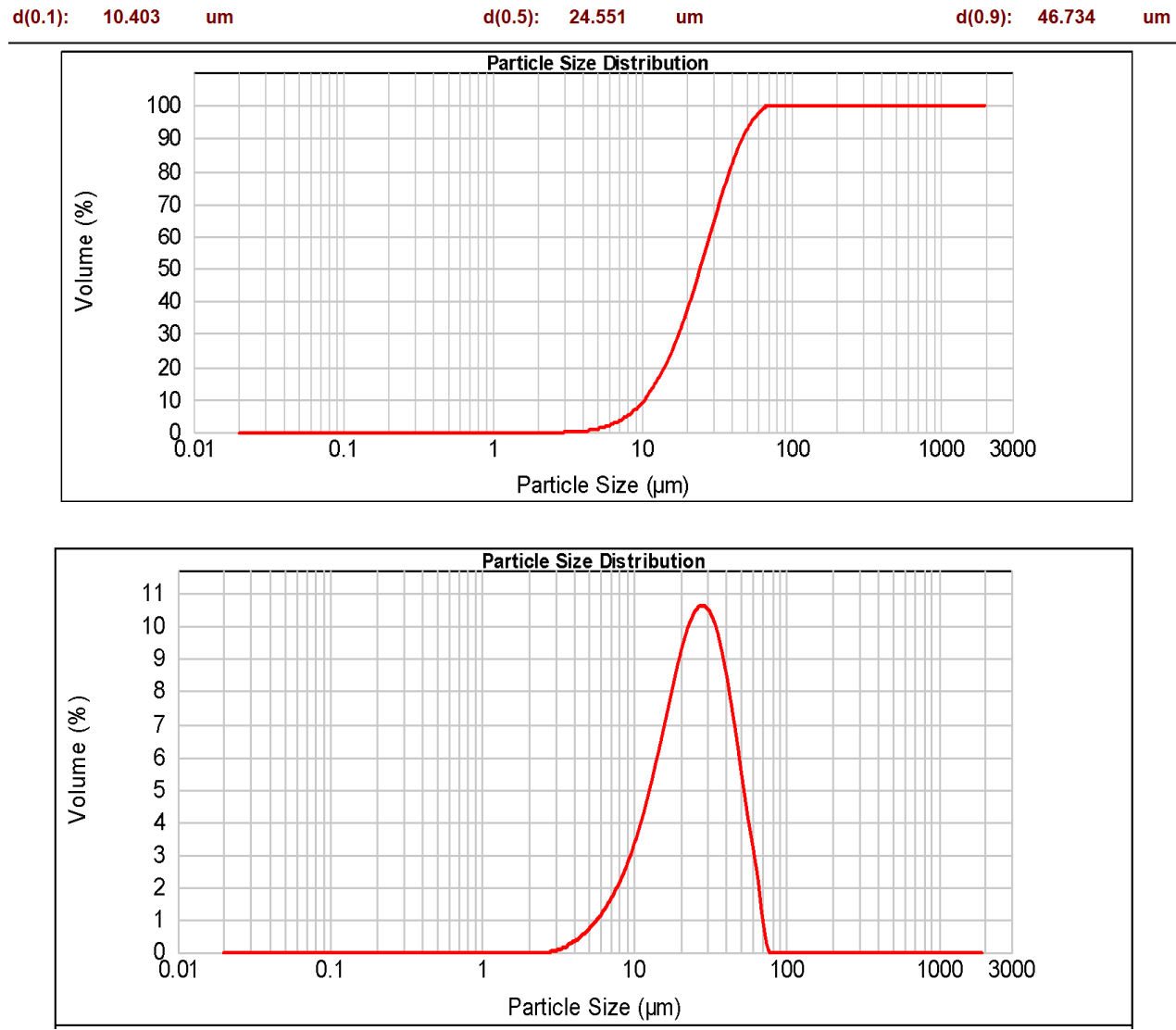

Figure 1: PSD of Gold (Au) Powder in Isopropanol

\subsection{Scanning Electron Microscope (SEM) Analysis of Gold (Au) Powder}

Figure 2 shows SEM images of gold powder. Gold ( $\mathrm{Au}$ ) powder was found to be mostly spherical in shape with smaller satellite particles agglomerated to the larger particles as shown in Figure 2c. 

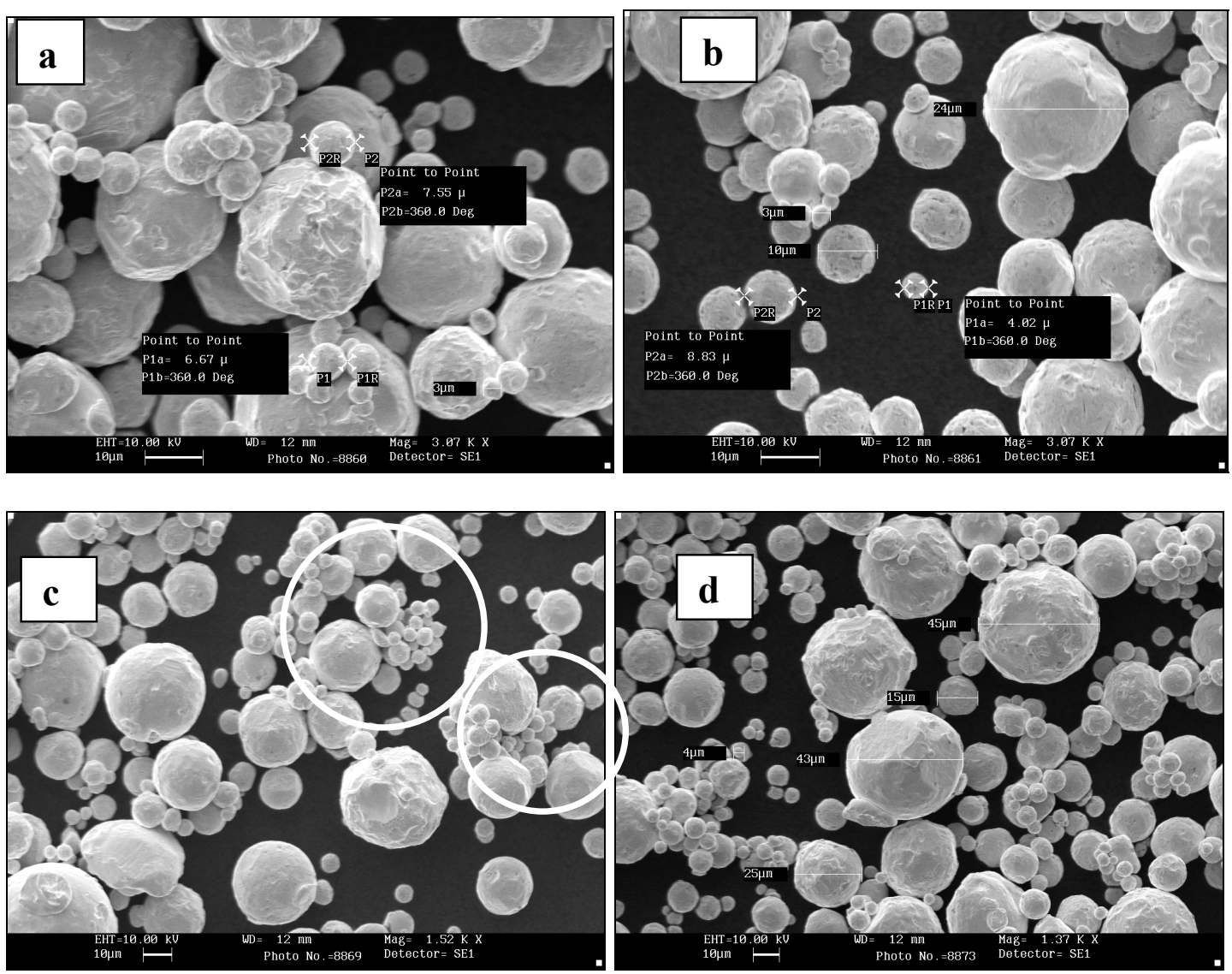

Figure 2 (a-d): SEM Images of Gold ( $\mathrm{Au})$ Powder

\subsection{Apparent Density Measurements}

The apparent density of powder is the density of powder in its loose state in a fixed size container [14]. Powder's apparent density or green density is of great importance in layer-based Rapid Manufacturing (RM) processes. Zhu et al [15] showed that apparent density or green density of the powder bed directly influences final density of the sintered parts. The higher the density of powder bed, then the higher will be the density of final sintered parts.

Here, gold powder was initially tested for apparent density according to ASTM B212-99 [16], ASTM B417-00 [17], BS EN 23923-1:1993 [18] and ISO 3923-1:1979 [19] standards. These standards use a Hall flowmeter funnel and Carney Funnel with an orifice of 2.5 and $5 \mathrm{~mm}$ respectively (depending on the flowability of metal powder through 2.5 or $5 \mathrm{~mm}$ orifice). It was found that gold powder did not flow freely though the Hall flowmeter funnel. In the Carney funnel, even with the assistance of a metallic wire through the funnel, gold powder was very reluctant to flow and would stop flowing as the metallic wire stopped moving. Therefore, ASTM B329 [20], BS EN 23923-2 [21] and ISO 3923-2 [22] standards were adopted which specify the apparent density measurement method for powder not flowing freely through a 2.5 or $5 \mathrm{~mm}$ orifice funnels. The equipment used for measuring apparent density of gold powder was the Scott Volumeter [23] (as shown in Figure 3). Apparent density was measured with the standard $25 \mathrm{~cm}^{3}$ density cup and two other cups with different height-to-diameter aspect ratios. These cups were used to check the variation in apparent density with change in the dimensions of the density cups. It is known that the pouring height (shielded and non-shielded) of the powder also influences its apparent density measurements [24], therefore gold powder was poured from a fixed height (as specified in the standards) for all measurements. 


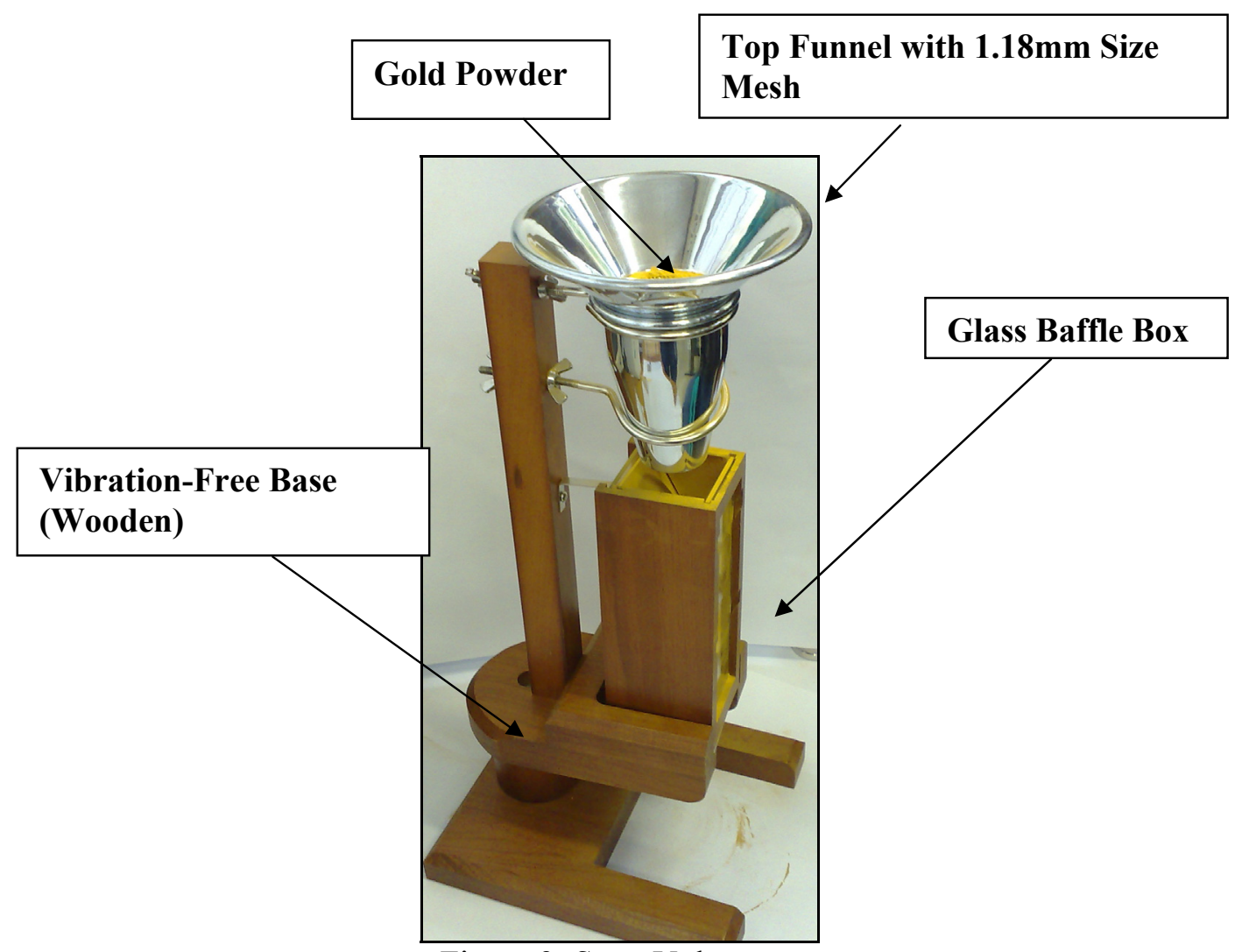

Figure 3: Scott Volumeter

The apparent density experiments were performed according to the standard and then a non-standard method. With the standard method, a $25 \mathrm{~cm}^{3}$ brass density cup was first weighed on a digital balance (accuracy of $\pm 0.05 \mathrm{~g}$ ). Gold powder was then added to the top hopper of the Scott volumeter and with the use of a nylon brush gold powder was assisted to flow through a $1.18 \mathrm{~mm}$ mesh size sieve. A glass slab was used to scrape the extra powder from the top of the $25 \mathrm{~cm}^{3}$ density cup as carefully as possible so as not to disturb the cup and weighed on a digital balance. The weight of the sample was calculated from the difference of the two readings (with and without metal powder).

With the non-standard method, apparent density experiments were also conducted with two stainless steel cups with different height-to-diameter aspect ratio. Table 1 specifies the aspect ratio and volume of all the density cups used to measure apparent density of gold powder including the standard $25 \mathrm{~cm}^{3}$ brass density cup.

Table 1: Aspect Ratio of Density Cups

\begin{tabular}{|c|c|c|c|}
\hline \multirow{2}{*}{ S/NO } & Aspect Ratio & Description & Volume $\left(\mathrm{cm}^{3}\right)$ \\
\hline Cup \# 1 & 0.5 & Stainless Steel Density Cup & 10 \\
\hline Cup \# 2 & 1.5 & Standard Brass Density Cup & 25 \\
\hline Cup \# 3 & 2 & Stainless Steel Density Cup & 9 \\
\hline
\end{tabular}

Figure 4 shows variation in the apparent density for different aspect ratio density cups. It was noted that these variations in the cup dimensions (height and diameter) had almost negligible effect on the apparent density of gold powder. 


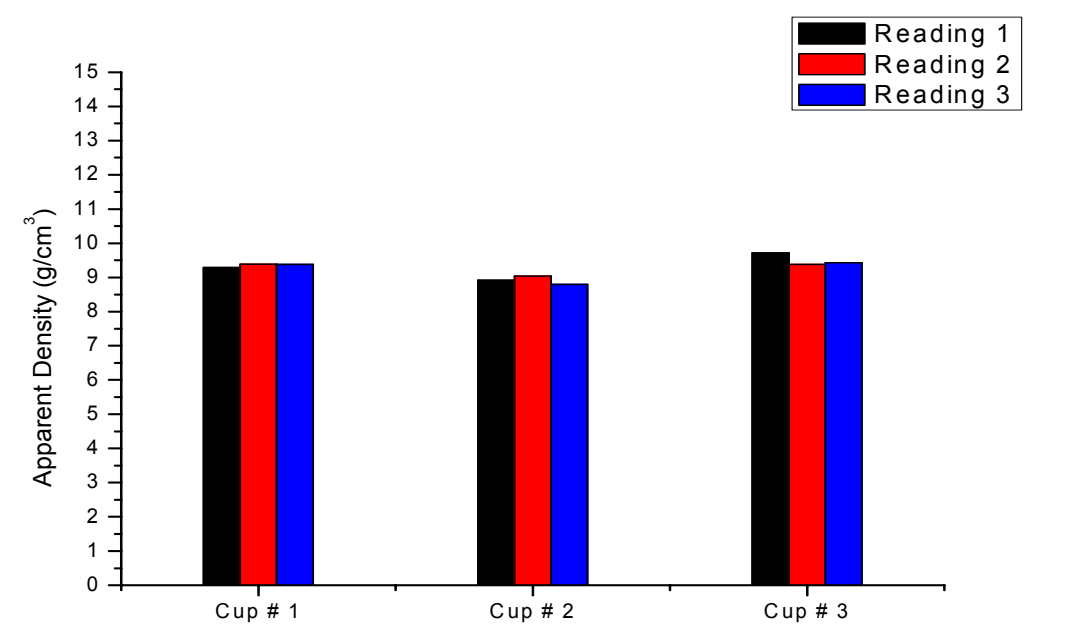

Figure 4: Apparent Density with Different Aspect Ratio Density Cups

\subsection{Tap Density Measurements}

The increase in the mean particle size has a great influence on the arrangement of particles as they try to form a dense packing [25]. During tapping or agitation of powder for compaction, smaller and larger particles re-arrange themselves to form a dense packing. Increasing the percentage of larger particles provide more inter-particle space for smaller particles to fill in. However, most of the smaller particles tend to go downward to the bottom of the density cup hence leaving more voids in the middle and upper section of the cup [26]. Tap density of the gold powder was determined by two different methods i.e. Constant Weight Tap Density (CWTD) method and Constant Volume Tap Density (CVTD) method.

\subsubsection{Constant Weight Tap Density (CWTD) Method}

The CWTD method is according to the ASTM B527 [27] and BS EN ISO 3953 [28] standards where tapping was performed by a manual method. As the apparent density of gold powder was greater than $7 \mathrm{~g} / \mathrm{cm}^{3}$, so according to tap density standards for metallic powders, the mass of the gold sample needed was $100 \mathrm{~g}$. A $100 \mathrm{~g}$ sample of gold powder was weighed on a digital balance (accuracy $\pm 0.05 \mathrm{~g}$ ) and poured gently into the standard 25 $\mathrm{cm}^{3}$ graduated glass cylinder to avoid any compaction of powder before tapping. It was also ensured that powder formed a horizontal level in the cylinder so that it could be easily measured. A total of 200 taps were performed for each sample with the manual method (according to BS EN ISO 3953:1995) using a hard rubber pad. The volume of the samples was measured directly from the readings on the standard $25 \mathrm{~cm}^{3}$ graduated cylinder to give the tap density after a specific number of taps. If the powder was not level in the cylinder then the average of the maximum and minimum value was taken as the tap density reading. Each of the experiments was performed three times to check repeatability of the results.

Figure 5 shows an increase in the tap density of gold powder with increasing number of taps for a standard $25 \mathrm{~cm}^{3}$ graduated glass cylinder. It can be noted that after 60 taps gold powder was almost completely compacted thereby reaching its maximum tap density. There was found to be very little variation in the tap density after 60 taps. Figure 5 follows a typical tap density curve where powder is compacted quickly in the initial taps while less variation is observed after it reaches its maximum compaction. 


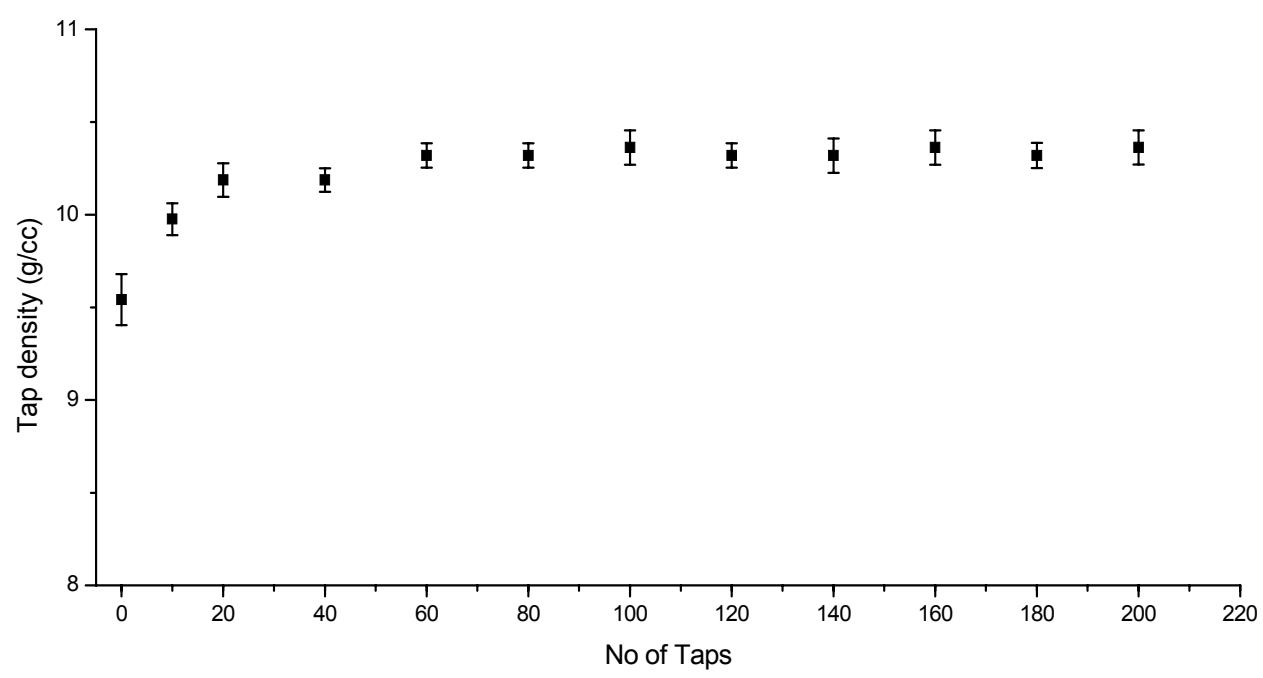

Figure 5: Tap Density of Gold Powder with CWTD Method

\subsubsection{Constant Volume Tap Density (CVTD) Method}

In contrast to the standard method of tap density measurement (CWTD method) in which mass of the test sample is fixed (100g in the case of gold powder), the CVTD method used a fixed volume of the test sample. In this method three density cups with different height-to-diameter aspect ratio (as shown in Table 1) were used to measure tap density, which were also used for apparent density measurements. All the density cups were weighed on a digital balance (accuracy of $\pm 0.05 \mathrm{~g}$ ). Powder was poured through Scott volumeter into the density cups. Excess powder was scraped with a glass slab and weighed. Extension pieces were placed and powder was added again from the Scott volumeter. The density cups were tapped and after removing the extensions the excess powder was scraped and the cups were weighed to give the tap density after specific number of taps. Figure 6 shows the CWTD of standard $25 \mathrm{~cm}^{3}$ graduated glass cylinder and CVTD of Cup \# 1, Cup \# 2 and Cup \# 3. It is noted that there is very little difference between the tap densities measured by CWTD and CVTD methods. Both in CWTD and CVTD methods, there is a jump between the first and second reading which indicates that gold powder compacted very quickly initially while after 60 taps the density remained almost constant.

- CVTD Cup \# 1

- CVTD Cup \# 2 (Standard $25 \mathrm{~cm}^{3}$ Density Cup)

- CVTD Cup \# 3

- CW TD Standard $25 \mathrm{~cm}^{3}$ graduated glass cylinder

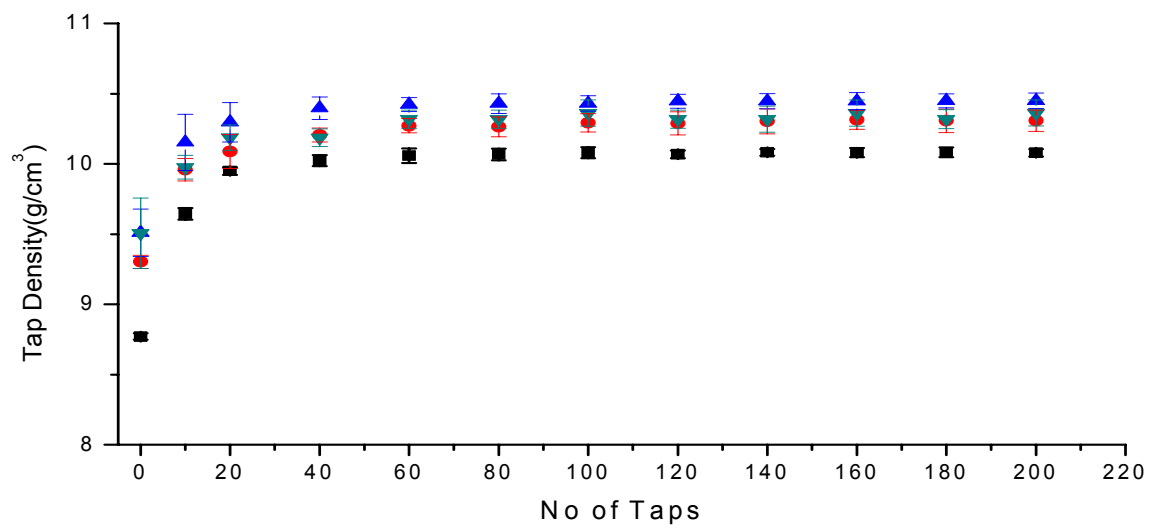

Figure 6: CVTD and CWTD for Different Density Cups 


\section{LASER SINTERING}

\subsection{Experimental setup}

A SLM 100 system was used to process gold powder. The SLM 100 system uses a 50 watts continuous wave ytterbium fibre laser at a wavelength of 1070 to $1090 \mathrm{~nm}$. The SLM 100's scanning system incorporates an f-theta lens and uses the same layered based techniques as the majority of the other SFF processes. In the SLM 100 a wiper spreads single layer of powder on the surface of a $125 \mathrm{~mm}$ diameter steel substrate followed by the fiber laser scanning a single cross-sectional layer of the part. This process is repeated multiple times to create 3 -dimesional metallic parts. Having a very small laser spot size (as small as $25 \mu \mathrm{m}$ ) and smaller build area, the SLM 100 is highly suitable for processing precious metals and alloys. The SLM 100 is also ideally suited for highly detailed and customized small parts such as jewellery items and dental crowns and implants etc. Figure 7 shows the SLM 100 setup.

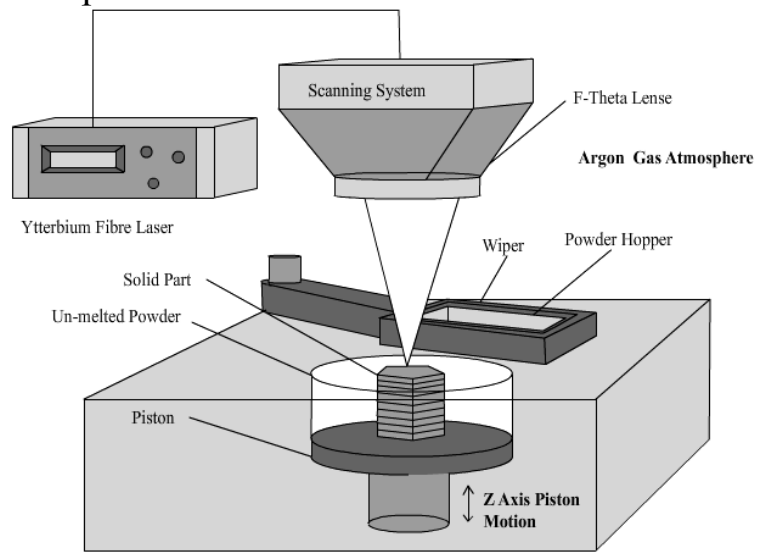

Figure 7: SLM 100 Setup

\subsection{Scan Strategy}

Processing of gold powder was carried out by two different scanning strategies. Figure $8 \mathrm{a}$ and $\mathrm{b}$ shows single-scan and cross-scan strategy for processing a single layer. In both the figures, a boundary scan (outer circle) was followed by a fill contour scan (inner concentric circle) while the solid area was scanned by hatch lines. In Figure 8a the whole area was scanned only once by single lines while in Figure $8 b$, the inner area was scanned two times by cross scanning.

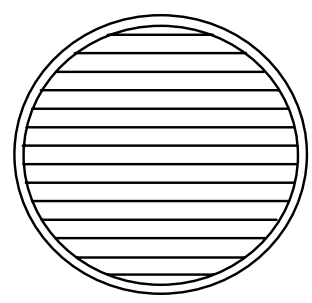

a

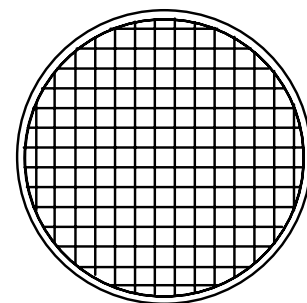

b

\subsection{Processing Parameters}

Figure 8: (a) Single-scan strategy and (b) Cross-scan strategy

A Laser power of 50 watts and hatch distance of $80 \mu \mathrm{m}$ was used in combination with different scanning speeds for boundary, fill contour and hatch solid to produce single layers. Processing parameters for gold $(\mathrm{Au})$ powder are summarized in Table 2. 
Table 2: Processing Parameters for Gold $(\mathrm{Au})$ Samples

\begin{tabular}{|l|l|l|}
\hline \multicolumn{2}{|l|}{ Processing Parameters } \\
\hline Laser power & Boundary solid & $\mathbf{5 0}$ Watts \\
\hline \multirow{3}{*}{ Scan speed } & Fill contour solid & $\mathbf{2 5 0} \mathrm{mm} / \mathrm{s}$ \\
\cline { 2 - 3 } & Hatch solid & $\mathbf{2 5 0} \mathrm{mm} / \mathrm{s}$ \\
\hline Percentage overlap & $\mathbf{1 5 0} \mathrm{mm} / \mathrm{s}$ \\
\hline Hatch distance & $\mathbf{7 2 \%}$ \\
\hline
\end{tabular}

\section{RESULTS AND DISCUSSIONS}

Different processing parameters such as laser power (watts), scan speed ( $\mathrm{mm} / \mathrm{s})$, hatch distance $(\mathrm{mm})$ and percentage overlap all hold an impact upon the formation of material processed using the SLM process. Figure 9 indicates the hatch scans, hatch distance, boundary scan and contour fill scan on the gold sample. Boundary scan represents the outer scan of each cross section of the part whereas contour fill scans represents all the inner scans parallel to the boundary scan. Boundary scan parameters influence the outer surface quality of the part in $\mathrm{z}$ direction.

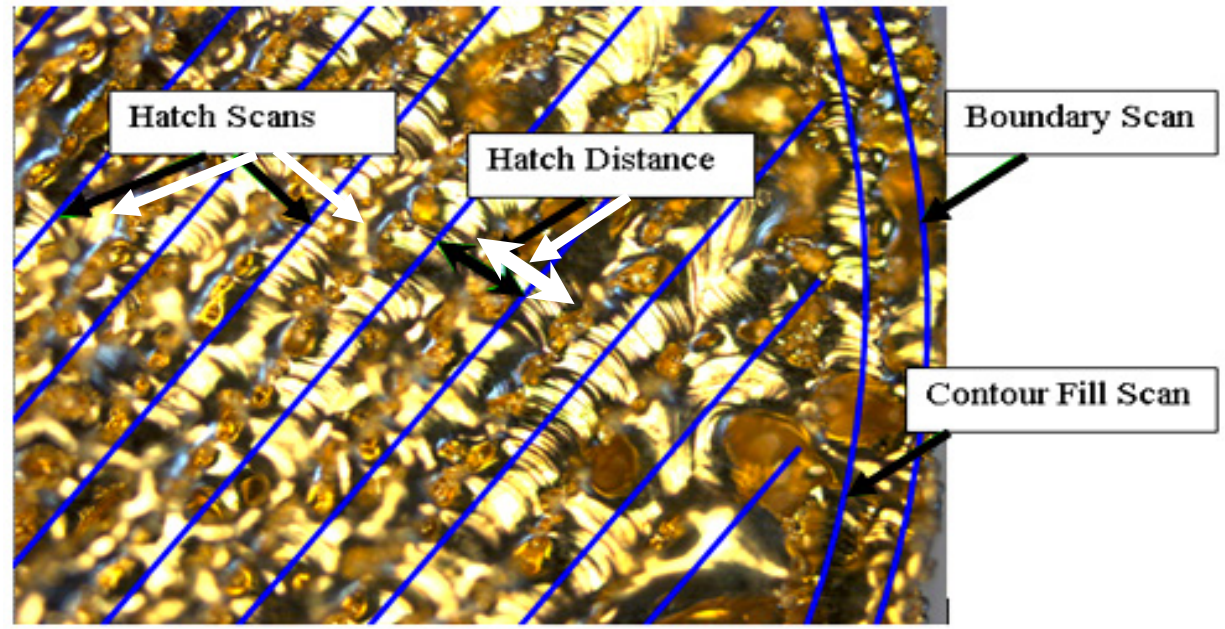

Figure 9: Optical Microscope image of single scan gold $(\mathrm{Au})$ layer
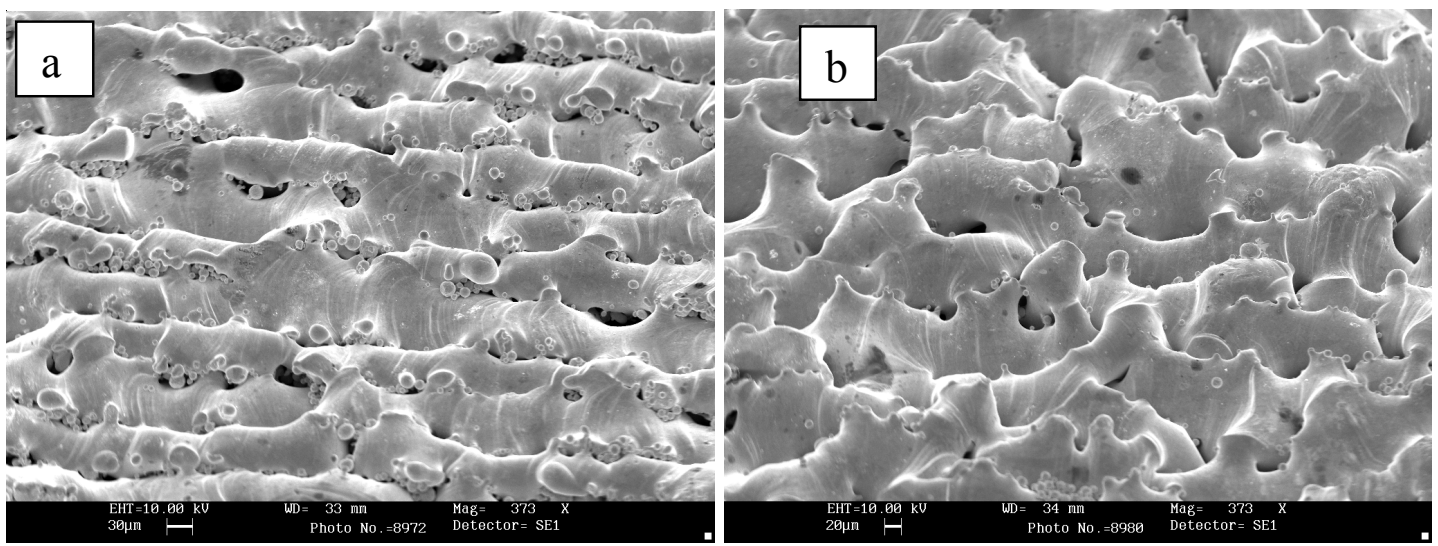

Figure 10: SEM images of scanned single layer of gold powder (a) single-scan strategy and

(b) cross-scan strategy

Figure 10 ( $a$ and $b$ ) shows SEM images of a single layer of gold after a single-scan and cross-scan strategy using the SLM 100 system. Here, gold particles were completely melted to form a solid structure. The interconnected porosity is quite evident from these images. It can be seen that a single-scan surface shows a much more linear structure as compared to the cross-scanned sample surface. Some gold particles were sticking to the melted lines in the single-scan strategy whereas in cross-scan strategy almost all the 
particles were completely melted. The cross-scanned surface was more irregular (greater number of peaks and valleys) as compared to the single-scan surface.

\subsection{Layer Thickness and Surface Morphology}

The majority of the Rapid Manufacturing processes are layer based processes and therefore quality of a single layer such as layer thickness and layer surface profile plays a vital role in the quality of the final product. If the initial layer in producing a component is irregular and non-uniform then the following layers will be distorted, which could result in problems like porous structures and layer de-bonding etc. Here, layer thickness measurements of single-scan and cross-scan gold samples were conducted using a Talysurf CLI 2000 (Taylor Hobson Ltd) and was taken as the average of the three readings. Layer thickness of cross-scan layer $(358 \mu \mathrm{m})$ was found to be much greater than single-scan layer $(278 \mu \mathrm{m})$ due to the extra powder melting on second laser scanning in the cross-scan strategy. Also, as gold samples were produced on a thick powder layer and in the crossscan strategy the same layer was scanned twice (with the same power and scan speed parameters) so more heat was dissipated into the powder thereby sintering more powder to the bottom side of the layer. Hence, this increased the layer thickness of the cross-scanned layer. Surface roughness measurements of the single-scan and cross-scan samples were performed using a Zygo and a Talysurf CLI 2000. A $300 \times 300 \mu \mathrm{m}$ area was scanned at three different points on the samples (as shown in Figure 11). Table 3 shows the average surface roughness of single-scan and cross-scan layers. There is not much difference between the surface roughnesses of the two scan strategies. The surface morphology is quite different as could be seen in Figure 10 where the single-scan strategy (Figure 10a) produces more uniform surface with low peaks (Figure 10b. Figure 11a and b also shows surface profile of the gold samples.

Table 3: Surface Roughness and Layer Thickness of Single-Scan and Cross-Scan Gold (Au) Samples

\begin{tabular}{|c|c|c|}
\hline Scan Strategy & Average Surface Roughness $(\boldsymbol{\mu m})$ & $\begin{array}{c}\text { Average Layer } \\
\text { Thickness }(\boldsymbol{\mu m})\end{array}$ \\
\hline Single Scan & 10.54 & 278 \\
\hline Cross Scan & 13.08 & 358 \\
\hline
\end{tabular}
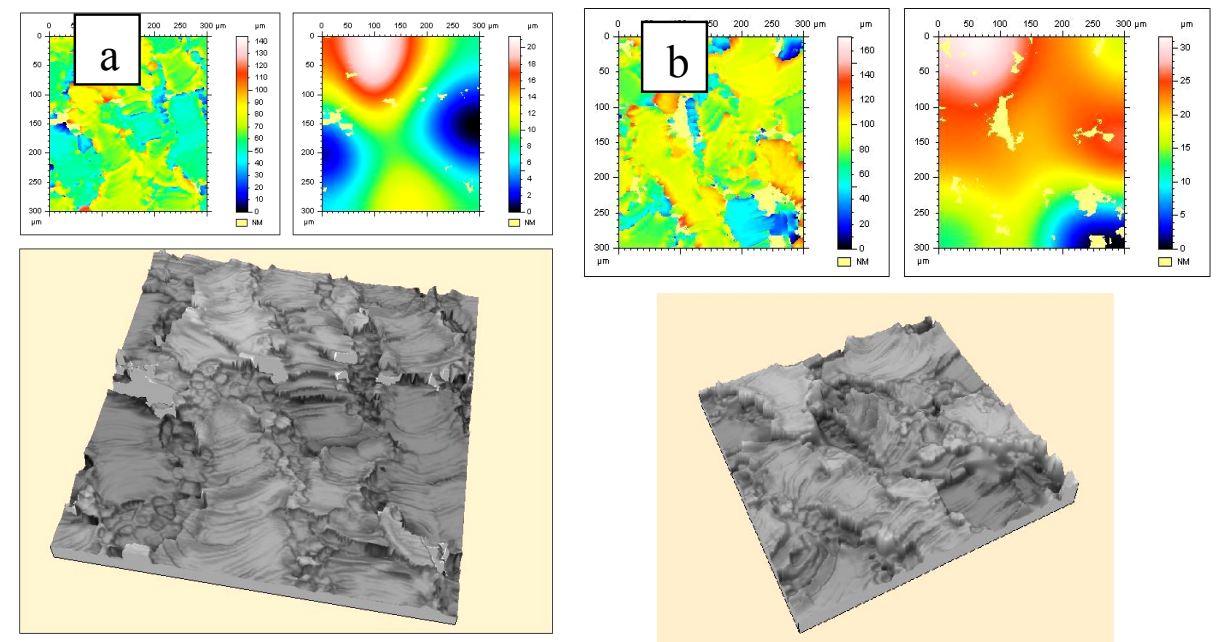

Figure 11: 3D surface of (a) single-scanned and (b) cross-scanned gold ( $\mathrm{Au}$ ) layers 


\section{CONCLUSIONS}

Initial investigation into the selective laser melting of gold powder was presented. Gold $(\mathrm{Au})$ powder was tested for apparent density, tap density and particle size distribution.

1. Gold powder was found to be less dispersing in water as compared to isopropanol for PSD analysis, which was also confirmed by the SEM images and mean particle sizes.

2. Gold powder was found to be cohesive in nature and very difficult to flow through Hall and Carney funnels.

3. Smaller variation in the height-to-diameter aspect ratio of density cups had negligible effect on the apparent density and tap density of gold powder.

4. Layer thickness of a cross-scanned layer was found to be significantly greater than the single-scanned layer.

5. Surface morphology of the scanned gold layers was found to be effected by the two scanning strategies, whereas surface roughness was almost the same.

\section{REFERENCES}

[1] Liao, H. T. and Shie J. R. (2007), Optimization on selective laser sintering of Metallic Powder via Design of Experiments Method, Rapid Prototyping Journal, Vol. 13, Issue 3, pp 156-162, ISSN: 1355-2546

[2] Keicher, D.M., Miller, W.D., Smugeresky, J.E. and Romero, J.A., (1998), Laser Engineered Net Shaping (LENS), Beyond Rapid Prototyping to Direct Fabrication, Proceedings of the TMS Annual Meeting, San Antonio, TX, pp. 369-377

[3] Lewis, G.K., Nemec, R., Milewski, J., Thoma, D.J., Cremers, D. and Barbe, M. (1994), Directed Light Fabrication, Proceedings of the International Congress on Applications of Lasers and Electro-Optics, Orlando, Florida, USA, pp. 17-26

[4] Childs, T.H.C., Hauser, C. and Badrossamay, M. (2005), Selective Laser Sintering (Melting) of Stainless and Tool Steel Powders: Experiments and Modelling, Proceeding of IMechE, Part B: Journal of Engineering Manufacture, Vol. 219, Issue 4, pp. 339-357, ISSN: 0954-4054

[5] Rombouts, M., Kruth, J. P., Froyen, L. and Mercelis, P. (2006), Fundamentals of Selective Laser Melting of alloyed steel powders, CIRP Annals - Manufacturing Technology, Vol. 55, Issue 1, pp. 187-192, ISSN: 0007-8506

[6] Kruth, J. P., Mercelis, P., Vaerenbergh van, J., Froyen, L. and Rombouts, M. (2004), Binding Mechanisms in Selective Laser Sintering and Selective Laser Melting, Rapid Prototyping Journal, Vol. 11, Issue 1, pp. 26-36, ISSN 13552546

[7] Gu, D. and Shen, Y. (2007), Balling Phenomena During Direct Laser Sintering of Multi-Component Cu-Based Metal Powder, Journal of Alloys and Compounds, Vol. 432, Issue 1-2, pp.163-166, ISSN: 0925-8388

[8] Mumtaz, K. A., Erasenthiran, P. and Hopkinson, N. (2008), High density selective laser melting of Waspaloy ${ }^{\circledR}$, Journal of Materials Processing Technology, Vol. 195, Issue 1-3, pp 77-87, ISSN: 0924-0136

[9] Yadroitsev, I., Thivillon, L., Bertrand, Ph. and Smurov, I. (2007), Strategy of manufacturing components with designed internal structure by selective laser melting of metallic powder, Applied Surface Science, Vol. 254, Issue 4, pp 980-983, ISSN: 0169-4332

[10] Simchi, A. (2006), Direct Laser Sintering of Metal Powders: Mechanism, Kinetics and Microstructural Features, Materials Science and Engineering: A, Vol. 428, Issue 1-2, pp. 148-158, ISSN 0921-5093

[11] Simchi, A. (2004), The Role of Particle Size on the Laser Sintering of Iron Powder, Metallurgical and Materials Transactions B, Vol. 35, Issue 5, pp. 937-948, ISSN $1543-1940$ 
[12] Fischer, P., Romano, V., Weber, H. P., Karapatis, N. P., Boillat, E. and Glardon, R. (2003), Sintering of Commercially Pure Titanium Powder with a Nd:YAG Laser Source, Acta Materialia, Vol. 51, Issue 6, pp. 1651-1662, ISSN 1359-6454

[13] Thorsson, L. (2006), Laser Sintering of Gold Jewellery, Proceeding of $1^{\text {st }}$ International Conference on Rapid Manufacturing, 5-6 July, Loughborough, UK

[14] German, R. M. (1989), Particle Packing Characteristics, Metal Powder Industry, Princeton, New Jersey, ISBN: 0-918404-83-5

[15] Zhu, H. H., Fuh, J. Y. H. and Lu, L. (2007), The Influence of Powder Apparent Density on the Density in Direct Laser-Sintered Metallic Parts, International Journal of Machine Tools and Manufacture, Vol.47, Issue 2, pp 294-298, ISSN: 0890-6955

[16] American Society for Testing Materials (1999), Standard Test Method for Apparent Density of Free Flowing Metal Powders using the Hall Flowmeter Funnel, ASTM B212 (re-approved 2006)

[17] American Society for Testing Materials (2006), Standard Test Method for Apparent Density of Non-Free-Flowing Metal Powders using the Carney Funnel, ASTM B 417

[18] British Standard (1993), Metallic Powders - Determination of Apparent DensityPart 1: Funnel Method, BS EN 23923-1

[19] International Organization for Standardization (1979), Metallic Powders Determination of Apparent Density - Part 1: Funnel Method, ISO 3923-1

[20] American Society for Testing Materials (1998), Standard Test Method for Apparent Density of Free Flowing Metal Powders using the Scott Volumeter, ASTM B329

[21] British Standard (1993), Metallic Powders - Determination of Apparent DensityPart 2: Scott Volumeter Method, BS EN 23923-2

[22] International Organization for Standardization (1981), Metallic Powders Determination of Apparent Density — Part 2: Scott Volumeter Method, ISO 3923-2

[23] Scott, G. D. (1960), Packing of equal spheres, Nature, Vol. 188, pp. 908-909, ISSN: 0028-0836

[24] Santomaso, A., Lazzaro, P. and Canu, P. (2003), Powder Flowability and Density Ratios: The Impact of Granules Packing, Chemical Engineering Science, Vol. 58, Issue 13, pp. 2857-2874, ISSN: 0009-2509

[25] Svarovsky, L. (1987), Powder Testing Guide: Methods of Measuring the Physical Properties of Bulk Powders, Elsevier Applied Science Publishers Ltd., London and New York, ISBN: 1-85166-137-9

[26] Abdullah E. C. and Geldart, D. (1999), The Use of Bulk Density Measurements as Flowability Indicators, Powder Technology, Vol. 102, pp. 151-165, ISSN: 00325910

[27] American Society for Testing Materials (2006), Standard Test Method for Determination of Tap Density of Metallic Powders and Compounds, ASTM B527

[28] British Standard (1995), Metallic Powders - Determination of Tap Density, BS EN ISO 3953 